\title{
The EBT-II Conceptual Design Study
}
F. W. Baity, Jr.
J. K. Ballou
D. B. Batchelor
E. S. Bettis
D. B. Campbell
G. L. Campen
K. H. Carpenter
J. A. Cobble
J. S. Culver
H. O. Eason
D. A. Everitt
W. M. Fletcher

$\quad$ R. A. Dandl
J. C. Glowienka
W. R. Hamilton
G. R. Haste
C. L. Hedrick
S. Hiroe
H. Ikegami
E. F. Jaeger
K. L. Kilby
S. P. Kuo
N. H. Lazar
J. N. Luton
J. A. Mayhall

R. A. Dandl

J. C. Glowienka

J. R. Moore

D. B. Nelson

L. W. Owen

G. F. Pierce

B. H. Quon

D. A. Spong

N. A. Uckan

H. L. Watts

T. L. White

R. A. Wright

W. L. Wright

J. W. Yarbrough 


\section{DISCLAIMER}

This report was prepared as an account of work sponsored by an agency of the United States Government. Neither the United States Government nor any agency Thereof, nor any of their employees, makes any warranty, express or implied, or assumes any legal liability or responsibility for the accuracy, completeness, or usefulness of any information, apparatus, product, or process disclosed, or represents that its use would not infringe privately owned rights. Reference herein to any specific commercial product, process, or service by trade name, trademark, manufacturer, or otherwise does not necessarily constitute or imply its endorsement, recommendation, or favoring by the United States Government or any agency thereof. The views and opinions of authors expressed herein do not necessarily state or reflect those of the United States Government or any agency thereof. 


\section{DISCLAIMER}

Portions of this document may be illegible in electronic image products. Images are produced from the best available original document. 
Printed in the United States of America. Available from National Technical Information Service

U.S. Department of Lommerce

5285 Port Royal Road, Springfield, Virginia 22161

Price: Printed Liopy $\$ y .00$; Mlcruliclie $\$ 3.00$

This report was prepared as an account of work sponsored by an agency of the United States Government. Neither the United States Government nor any agency thereof, nor any of their employees, contractors, subcontractors, or their employees, makes any warranty, express or implied, nor assumes any legal liability or responsibility for any third party's use or the results of such use of any information, apparatus, product or process disclosed in this report, nor represents that its use by such third party would not infringe privately owned rights. 
ORNL/TM-5955

Dist. Category UC-20 f, $\mathrm{g}$

Contract No. W-7405-eng-26

FUSION ENERGY DIVISION

THE EBT-II CONCEPTUAL DESIGN STUDY

\section{R. A. Dand 1}

\begin{tabular}{|c|c|c|}
\hline F. W. Baity, Jr. & J. C. Glowienka & J. R. Moore ${ }^{2}$ \\
\hline J. K. Ballou & W. R. Hami 1 ton $^{2}$ & D. B. Nelson \\
\hline D. B. Batchelor & G. R. Haste & L. W. Owen ${ }^{3}$ \\
\hline E. S. Bettis ${ }^{1}$ & C. L. Hedrick & G. F. Pierce 2 \\
\hline D. B. Campbel1 ${ }^{2}$ & S. Hiroe 4 & B. H. Quon \\
\hline G. L. Campen ${ }^{2}$ & H. Ikegami ${ }^{4}$ & D. A. Spong \\
\hline K. H. Carpenter ${ }^{3}$ & E. F. Jaeger & N. A. Uckan \\
\hline J. A. Cobble & K. L. Kilby ${ }^{2}$ & H. L. Watts ${ }^{2}$ \\
\hline J. S. Culver 2 & S. P. Kuo 5 & T. L. White \\
\hline H. 0. Eason & N. H. Lazar & R. A. Wright ${ }^{2}$ \\
\hline D. A. Everitt ${ }^{2}$ & J. N. Luton & W. L. Wright ${ }^{2}$ \\
\hline W. M. Fletcher 2 & J. A. Mayha $11^{2}$ & J. W. Yarbrough ${ }^{2}$ \\
\hline
\end{tabular}

${ }^{1}$ Consultant.

${ }^{2}$ UCC-ND Engineering.

${ }^{3}$ Computer Sciences Division.

${ }^{4}$ Consultant, Institute of Plasma Physics, Nagoya University, Nagoya, Japan.

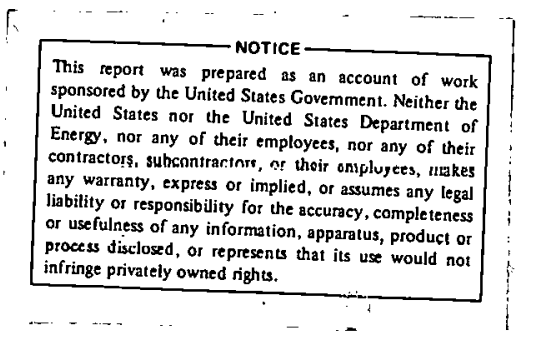

${ }^{5}$ Consultant, Rensselaer Polytechnic Institute, Troy, New York.

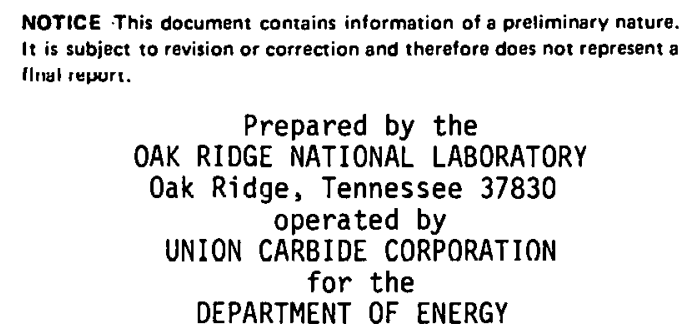


THIS PAGE

WAS INTENTIONALLY

LEFT BLANK 
ABSTRACT

Experiments in the ELMO Bumpy Torus (EBT-I) have demonstrated that plasma currents produced by microwave heated, hot electron annuli can provide macroscopically stable plasma confinement in a steady-state bumpy torus. The EBT experiments have established a reference case in which the data agree sufficiently well with neoclassical transport theory to make imperative a full test of the transport scaling. If the EBT approach can be extended to confine high beta toroidal plasmas, it would, in its high aspect ratio, conveniently accessible, steady-state reactor embodiment, offer an attractive alternative to the tokamak and magnetic mirror approaches to controlled thermonuclear fusion.

Our present understanding of equilibrium, stability, and transport in EBT has suggested a sequence of experimental devices and related research activities leading progressively toward an attractive full-scale reactor. The implementation of the steps in this sequence hinges on the development of very high power continuous wave $(\mathrm{cw})$ microwave sources, at millimeter wavelengths.

A new device, the EBT-II proposed here, would provide a test of scaling, accelerate the development of the technology required for later reactor applications, provide a demonstration of the capability to analyze and control higher beta plasmas, test theoretical studies of EBT transport, and provide a focus for reactor applications design studies.

The proposed EBT-II will use 48 superconducting coils to provide the required steady-state, cyclotron resonant, toroidal magnetic field of $4.3 \mathrm{~T}$ for electron cyclotron heating (ECH) with microwaves at $120 \mathrm{GHz}$. The peak design fields are $7.8 \mathrm{~T}$ in the coil, $6.0 \mathrm{~T}$ in the coil throat, and $3.0 \mathrm{~T}$ in the cavity midplane. Bulk ECH will be provided by eight 120-GHz, 200-kW cW gyrotrons, and provisions have been made for an additional $800 \mathrm{~kW}$ of $\mathrm{cW}$ profile heating in the range of 70-90 GHz. The torus major radius and mean coil radius are $520 \mathrm{~cm}$ and $28 \mathrm{~cm}$ respectively, yielding a mechanical aspect ratio of approximately 20 . The basic device parameters of microwave frequency and power, magnetic field, and size should allow experiments that penetrate well into the collisionless regime and thus provide a definitive test of neoclassical scaling. This scaling test will be pushed further by using a built-in provision which permits changing the effective aspect ratio by varying the current through 96 copper toroidal aspect ratio enhancement (ARE) coils ( 2 per microwave cavity).

Phase 1 of the experiment will be the study of transport and aspect ratio scaling of basic device parameters for comparison with results from EBT-I and EBT-SCale (EBT-S). Under optimum conditions, we project $n_{\tau} \sim 10^{13} \mathrm{sec} / \mathrm{cm}^{3}$ with $T_{j}$ and $T_{e} \sim 3-8 \mathrm{keV}$. Phase 2 will center on the addition of $1 \mathrm{MW}$ of neutral beam heating for direct heating of ions in conjunction with microwave heating of electrons so as to permit control of the ambipolar potential. With this added heating power, it appears possible to attain near-breakeven conditions in a hydrogen plasma at $\beta \approx 10 \%$. 


\section{THIS PAGE}

\section{WAS INTENTIONALLY}

\section{LEFT BLANK}




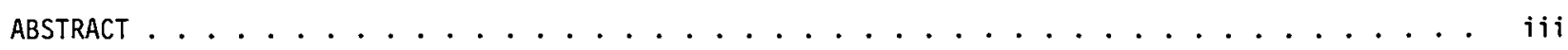

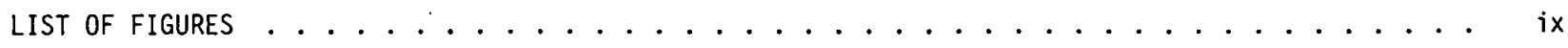

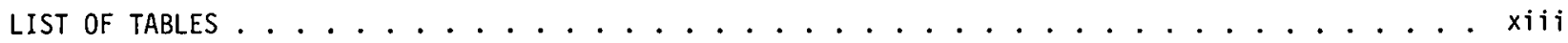

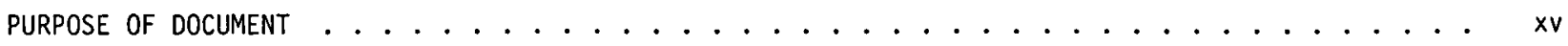

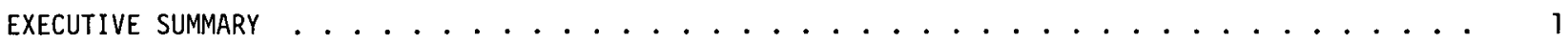

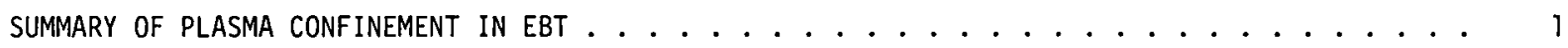

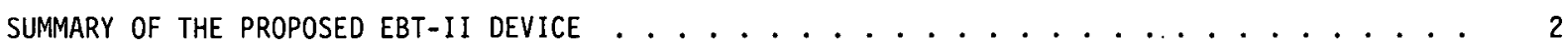

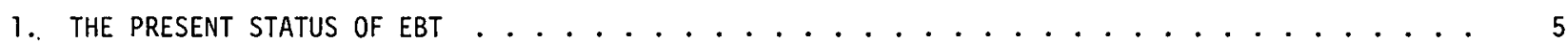

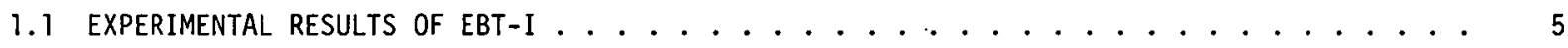

1.1 .1 Electron Temperature . . . . . . . . . . . . . . . . . 7

1.1 .2 Ion Energy . . . . . . . . . . . . . . . . . 7

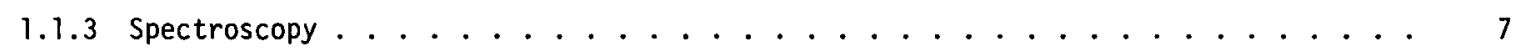

1.1 .4 Plasma Potential Measurement .................. 7

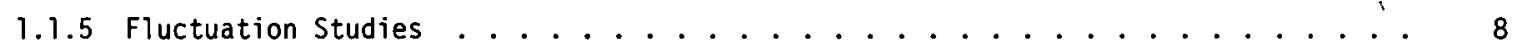

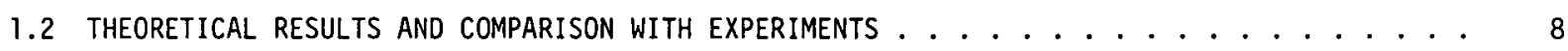

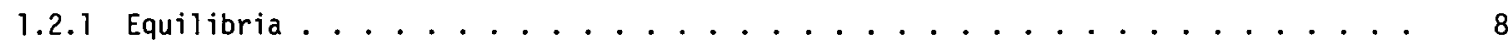

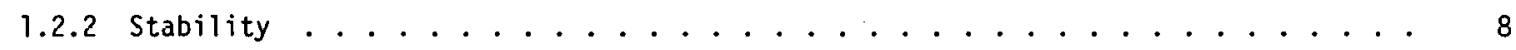

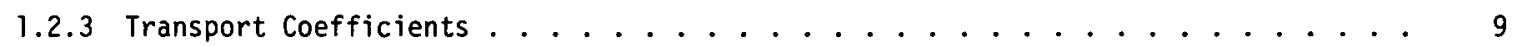

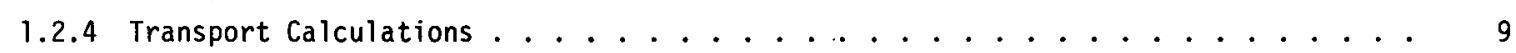

1.2 .5 Electron Heating and Microwave Propagation . . . . . . . . . . . . 9

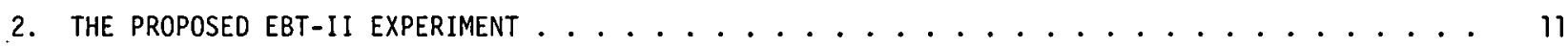

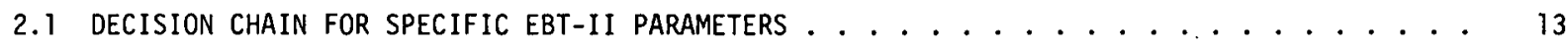

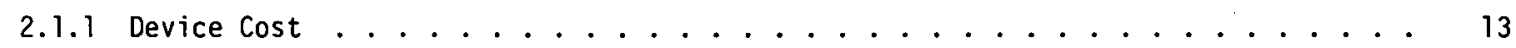

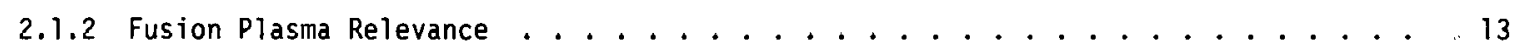

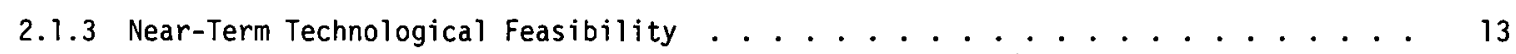

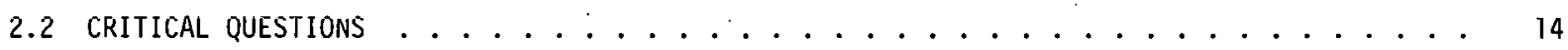

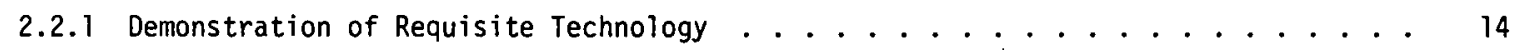

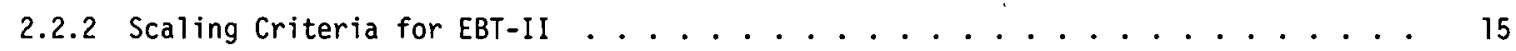

2.2.3 Confinement at Higher Values of Beta ................. 18

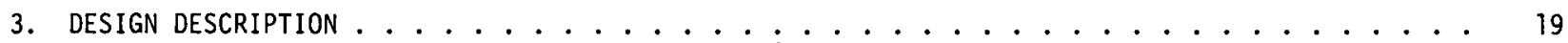

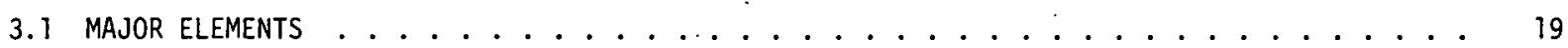

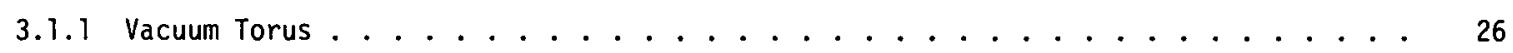

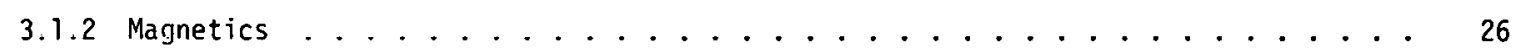

3.1.3 Vacuum Pumping Systems ...................... 48

3.1 .4 Microwave Heating System .................... . . 49

3.1 .5 Neutral Beam Heating Systems .................... 52

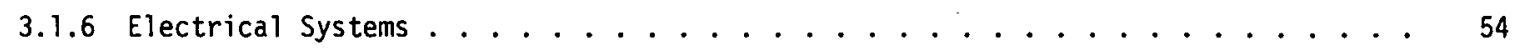

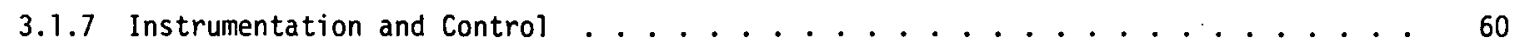

3.1 .8 structural Considerations . . . . . . . . . . . . . . 70

3.1 .9 Mechanical systems .......................... 75

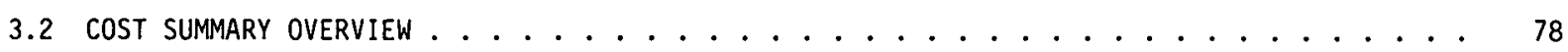

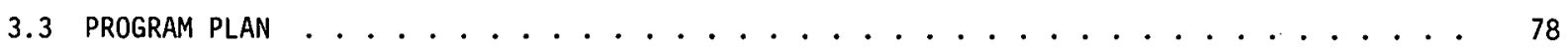




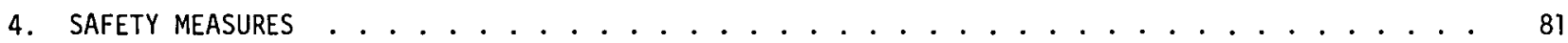

4.1 SAFETY ACTIVITIES . . . . . . . . . . . . . . . . . . . . . . 81

4.1.1 Division and Plant Safety Activities. . . . . . . . . . . . . . . . . . . 81

4.1.2 Department of Energy Safety Requirements . . . . . . . . . . . . . . . . 81

4.1 .3 Preparatory Safety Activities . . . . . . . . . . . . . . . . . . 81

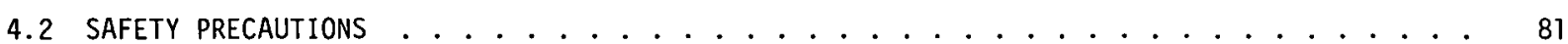

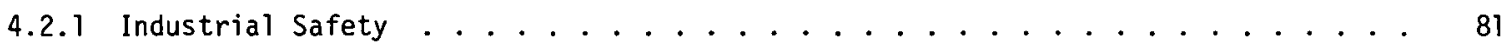

4.2.2 Microwaye Sạfeţy . . . . . . . . . . . . . . . . . . . . . . . . . 82

4.2.3 Seismic Considerations . . . . . . . . . . . . . . . . . . . . 82

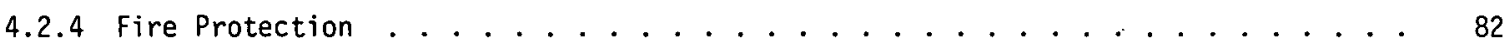

4.3 RADIOLOGICAL SAFETY . . . . . . . . . . . . . . . . . . . . . . 82

4.3.1 Hydrogen Plasma . . . . . . . . . . . . . . . . . . . . . . . 83

4.3 .2 Deuterium Plasma . . . . . . . . . . . . . . . . . . . . . . 83

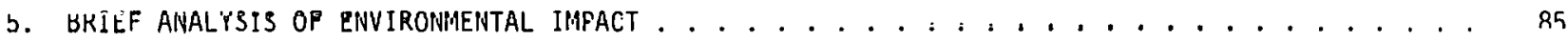

APPENDIXES . . . . . . . . . . . . . . . . . . . . . . . . . . . 87

A. STABILITY THEORY . . . . . . . . . . . . . . . . . . . . . . . . . . 87

A. 1 MAGNETOHYDRODYNAMICS . . . . . . . . . . . . . . . . . . . . . . . . . . . . . 90

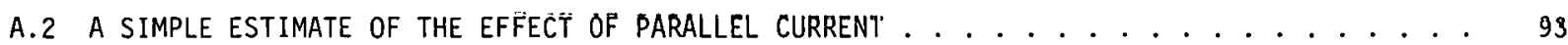

A.3 STABILITY OF THE HUT ELECTRON RINGS . . . . . . . . . . . . . . . . . . . . . . . . . 95

A.4 DISSIPATIVE AND OTHER INSTABILITY DRIVES . . . . . . . . . . . . . . . . . . . . . 96

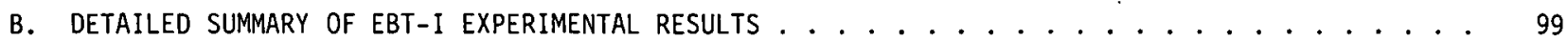

B. 1 PHYSICAL DESCRIPTION AND OPERATION . . . . . . . . . . . . . . . . . . . . 101

B.2 EARLY EXPERIMENTS IN EBT-I . . . . . . . . . . . . . . . . . . . . . . 106

B.2.1 DEMONSTRATION OF VALIDITY OF EBT CONCEP' . . . . . . . . . . . . . . . . . 106

B.2.2 MEASUREMENT OF THE LOCATION OF THE TOROIUAL PLASMA . . . . . . . . . . . . . . 106

B.3 STABILITY OF THE ELECTRON NNNULUS . . . . . . . . . . . . . . . . . . . . . . . IIU

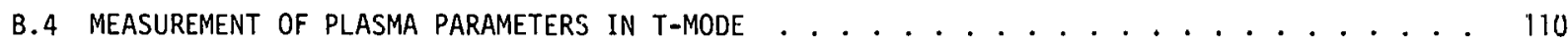

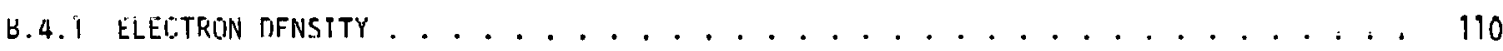

B.4.2 ElECTRON TEMPERATURE . . . . . . . . . . . . . . . . . . . . . . . 114

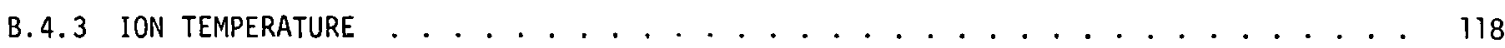

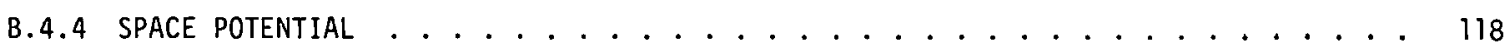

B.4.5 IMPURITIES . . . . . . . . . . . . . . . . . . . . . . . . . . 122

B. 4.6 NEUTRAL HYDROGEN . . . . . . . . . . . . . . . . . . . . . . . . . . . 129

B.4.7 TOROIDAL CURRENTS AND FIELD ERROR EFFECTS ON EBT . . . . . . . . . . . . . . . . 132

C. EBT-II MAGNETICS DESIGN . . . . . . . . . . . . . . . . . . . . . . . 141

D. NEUTRAL BEAM HEATING FOR EbT-II . . . . . . . . . . . . . . . . . . . . . . . . 151

D. 1 Plasma parameters relevant to neutrnl beam heating . . . . . . . . . . . . . . . 153

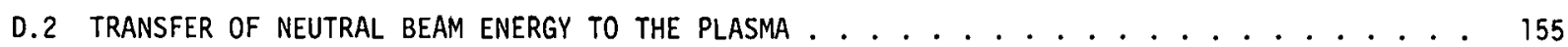

D. 3 VACUUM SYSTEM REQUIREMENTS FOR STEADY-STATE INJECTION . . . . . . . . . . . . . . 159

D.4 BEAM SYSTEM SPECIFICATIONS . . . . . . . . . . . . . . . . . . . . . 160

E. RELEVANCE OF THE EBT-II EXPERIMENT TO AN EBT REACTOR . . . . . . . . . . . . . . . . . . 163

E.1 PROPERTIES OF THE EBT REACTOR . . . . . . . . . . . . . . . . . . . . . . 166

E.1.1 POWER DENSITY SCALING . . . . . . . . . . . . . . . . . . . . . . 166

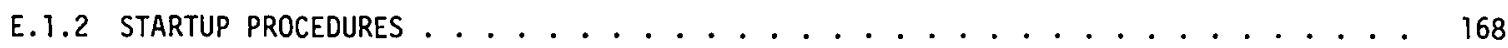


E. 1.3 MAINTAINING STEAdY STATE . . . . . . . . . . . . . . . . . . . . . . . . 168

E.1.4 RECIRCULATING POWER FRACTION . . . . . . . . . . . . . . . . . . . . . . . 168

E. 1.5 OVERALL PLANT DESIGN . . . . . . . . . . . . . . . . . . . . . . . . . 169

E.2 SEQUENCE OF EBT DEVICES . . . . . . . . . . . . . . . . . . . . . . . 172

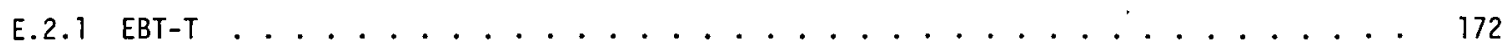

E.2.2 EBT-IT . . . . . . . . . . . . . . . . . . . . . . . . 172

E.2.3 EBT-R . . . . . . . . . . . . . . . . . . . . . . . . . . . 173

E.3 RESEARCH, DEVELOPMENT, AND DEMONSTRATION NEEDS . . . . . . . . . . . . . . . . . . . 173

E.3.1 PLASMA PHYSICS NEEDS . . . . . . . . . . . . . . . . . . . . . . . . 173

E.3.2 SYSTEM STUDIES . . . . . . . . . . . . . . . . . . . . . . . . . . . . 174

E.3.3 MICROWAVE DEVELOPMENT . . . . . . . . . . . . . . . . . . . . . . . 174

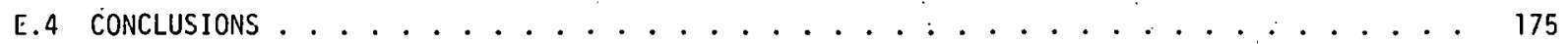


THIS PAGE

\section{WAS INTENTIONALLY \\ LEFT BLANK}


1.1 Schematic layout of EBT-II . . . . . . . . . . . . . . . . . . . 6

3.1 EBT-IT project location ............................ 20

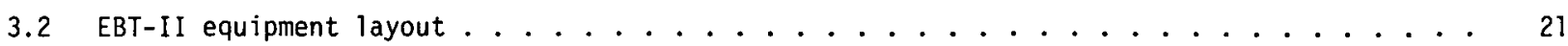

3.3 EBT-II device plan view . . . . . . . . . . . . . . . . . . . . . 22

3.4 Section elevation through typical cavity . . . . . . . . . . . . . . . . . . 23

3.5 Section elevation through neutral beam input cavity ................... 24

3.6 Section plan through torus ............................ 27

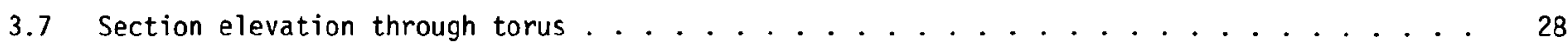

3.8 ARE coil effect on magnetic flux lines ....................... 29

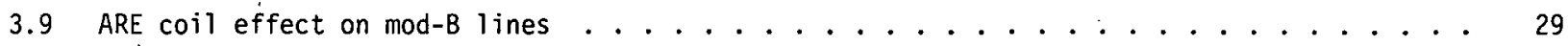

3.10 Section plan through mirror coil . . . . . . . . . . . . . . . . . . . . . 31

3.11 Section elevation through mirror coil . . . . . . . . . . . . . . . . . . 32

3.12 Definition of shielding calculation nomenclature . . . . . . . . . . . . . . . 35

3.13 Plan view of ARE coil restraint system . . . . . . . . . . . . . . . . . 37

3.14 Elevation of ARE coil restraint system . . . . . . . . . . . . . . . . . . . 38

3.15 Mirror coil magnetic load distribution . . . . . . . . . . . . . . . . . . 40

3.16 ARE coil magnetic load distribution .......................... 41

3.17 Finite element model of ARE coil . . . . . . . . . . . . . . . . . . . 43

3.18 Deflected ARE coil model . . . . . . . . . . . . . . . . . . . . . . 44

3.19 Reactions at ARE coil support locations .......................... 45

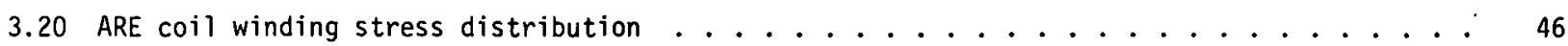

3.21 ARE coil case stress distribution . . . . . . . . . . . . . . . . . . . . 46

3.22 Vacuum system flow diagram ........................... 50

3.23 Switch gear diagram . . . . . . . . . . . . . . . . . . . . . . 55

3.24 Gyrotron power supply schematic . . . . . . . . . . . . . . . . . . . . . 57

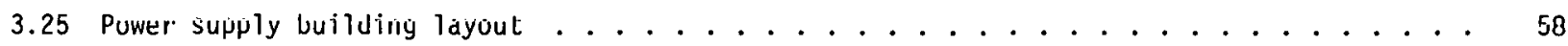

3.26 Neutral beam power supply schematic . . . . . . . . . . . . . . . . . . . 59

3.27 Vacuum system instrumentation diagram ......................... 63

3.28 Fluid flow instrumentation diagram . . . . . . . . . . . . . . . . . . 65

3.29 Neutral beam line instrumentation diagram . . . . . . . . . . . . . . . . . 66

3.30 ARE coil instrumentation schematic . . . . . . . . . . . . . . . . . . 68

3.31 Mirror coll instrumentation schematic . . . . . . . . . . . . . . . . . 68

3.32 Data acquisition system schematic . . . . . . . . . . . . . . . . . . 71

3.33 Data system schematic . . . . . . . . . . . . . . . . . . . . . . 72

3.34 Helium refrigerator equipment location . . . . . . . . . . . . . . . . . . 74

3.35 Outside equipment location . . . . . . . . . . . . . . . . . . . . 76

3.36 Fluids flow system schematic . . . . . . . . . . . . . . . . . . . 77

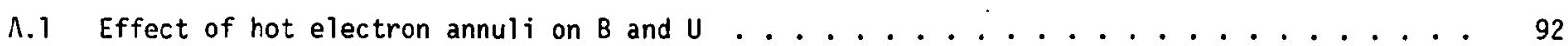

A.2. Maximum stable toroidal beta as a function of $B_{\text {annulus for a long annulus }}$

A.3 Comparison of densities and temperatures for annulus and toroidal core plasma . . . . . . 97

B.1 Schematic layout of EBT-I . . . . . . . . . . . . . . . . . . . . 102

B.2 Photograph of EBT-I . . . . . . . . . . . . . . . . . . . . . 103

B.3 Section through EBT-I in the horizontal midplane . . . . . . . . . . . . . . . 105

8.4 Data sheet of September 13, 1973 showing $950 \mathrm{~W}$ of $18-\mathrm{GHz}$ power delivered
to each of four toruidal sectbrs . . . . . . . . . . . . . . . . . . 107 
B.5 Plasma parameters measured in EBT-I as a function of observed stored energy $W_{\perp} . . . . .108$

B.6 Measurements of location of annulus with various magnetic field strengths using a skimmer probe containing an ionization chamber to intercept energetic electrons . . . . 109

B.7 Location of electron annulus from data in Fig. B.3 as a function of magnetic

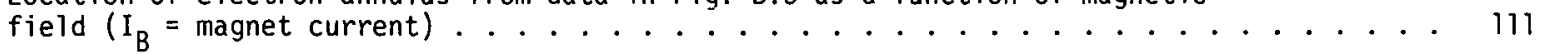

B.8 Saturation ion current measured with Langmuir probes as a function of radius . . . . . 112

B.9 Tracing of decays of $W_{1}$ (measured with diamagnetic loop) and $n_{e^{l}}$ (measured with interferometer) following turnoff of microwave power . . . . . . . . . . . . 113

B.10 Receiver optics for Thomson scattering measurement of ruby laser light at 90 . . . . . 115

B.11 Geometry used with Si(Li) detector for viewing bremsstrahlung in EBT-I . . . . . . . 115

B.12 Count rate spectrum obtained with $\mathrm{Si}(\mathrm{LI})$ detector collimated to view line

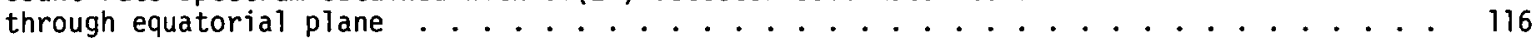

B.13 Count rate distribution obtained with $\mathrm{Si}(\mathrm{Li})$ detector after correction

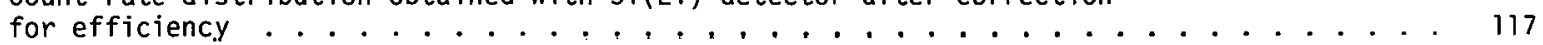

B.14 Schematic arrangement of system used to detect charge exchange neutral particles from EBT-I ............................. . . . . . . . . . . . 9

B.15 Efficiency of nitrogen stripping cell and cesium heat pipe charge exchange cell as a function of incident neutral particle energy in geometry used

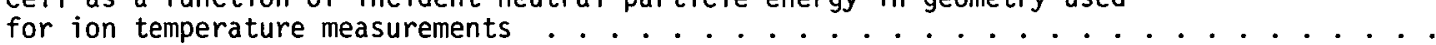

B. 16 Typical count rate spectrum of neutral particle energy obtained with (a) the nitrogen and (b) the cesium spectrometers under T-mode conditions (microwave power: $40 \mathrm{~kW}$ at $18 \mathrm{GHz}, 8 \mathrm{~kW}$ at $10.6 \mathrm{GHz}$; midplane magnetic field, $5 \mathrm{kG}$; neutral pressure, $5.1 \times 10^{-6}$ torr

B.17 Schematic drawing showing path of injected $\mathrm{Rb}^{+}$ions and orbits of secondarily ionized $\mathrm{Rb}^{++}$ions from various points along beam path . . . . . . . . . . . . . . . 123

B. 18. General layout of heavy ion beam probe system used in EBT-I . . . . . . . . . . . . . . 124

B.19 Space potential measured in EBT-I as a function of position in the midplane (T-mode conditions) . . . . . . . . . . . . . . . . . . . . . . 125

B.20 Space potential measured in EBT-I as a function of position in midplane

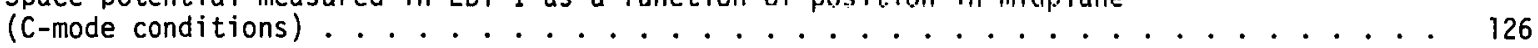

B.21 Geometry used for spatial scanninq of uv light from plasma in midplane nf EBT-I. . . . . . 127

B.22 Light intensities from $A$ I I at $\lambda=2151 \AA$ as a function of angle of view $(\theta)$ from top (RCY) and side (RCX) ports......................130

B.23 Light intensities from AI II at $\lambda=1671 \AA$ and reconstructed emission distrihution

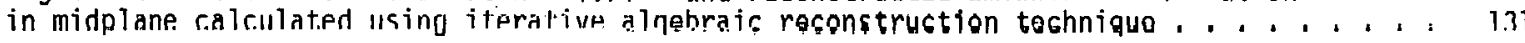

B.24 Wavelength scan of $H_{\alpha}$ light using Fabry-Perot interferometer . . . . . . . . . . . . . 133

B.25 Light emission profiles from hydrogen atoms $(H I)$ at $\lambda=1216 \AA$ (see Fig. B.13 for definitions) ............................ 134

B.26 Schematic section of EBT-I showing locations of quadripolar correction coils

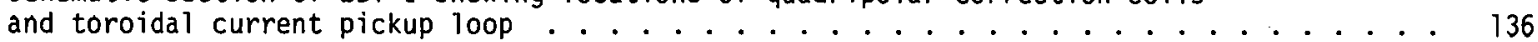

B.27 Toroidal current measured with pickup loop as a function of horizontal error fiel.d current for plasmas produced at various pressures.... . . . . . . . . . . 136

B.28 Correlations of several parameters measured simultaneously as the horizontal field error scans in the ranqe of $\pm 1-2 \times 10^{-3}$

C.1 Area of last closed drift orbit that does not intercept the limiter (mirror coil case) plotted as a function of the cosine of the pitch angle

for a 36-sector device ...............................

C.2 Area of last closed drift orbit that does not intercept the limiter (mirror coil case) plotted as a function of the cosine of the pitch angle........... 115

C. 3 Sector of EBT-II in the equatorial plane displaying position of the superconducting mirror coils and AR[ coil3 ...................... . . . . . . . . . .

C.4 Effect of ARE coils on field geometry ....................... . 148

C.5 Effects of ARE coils on particle drift orbits for $v_{\| l} / v=0$. . . . . . . . . . . . 149

C.6 Effects of ARE coils on particle drift orbits for $v_{\|} / v=1.0 \ldots \ldots$ 
D.1 Side view of torus showing vertical injection geometry . . . . . . . . . . . . . 154

D.2 Separated and total cross sections for initial collision of injected neutral.

beam vs energy . . . . . . . . . . . . . . . . . . . . . . . . . . . . . 156

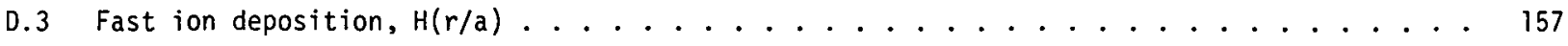

D.4 Beam power and current required to deposit $300 \mathrm{~kW}$ in the plasma vs beam energy . . . . 158

E.1 Relationship between plasma, magnetic field, and power production parameters ....... 167

E.2 EBTR plan view ........................... . . . . . . . . . . . . . . . . . . 


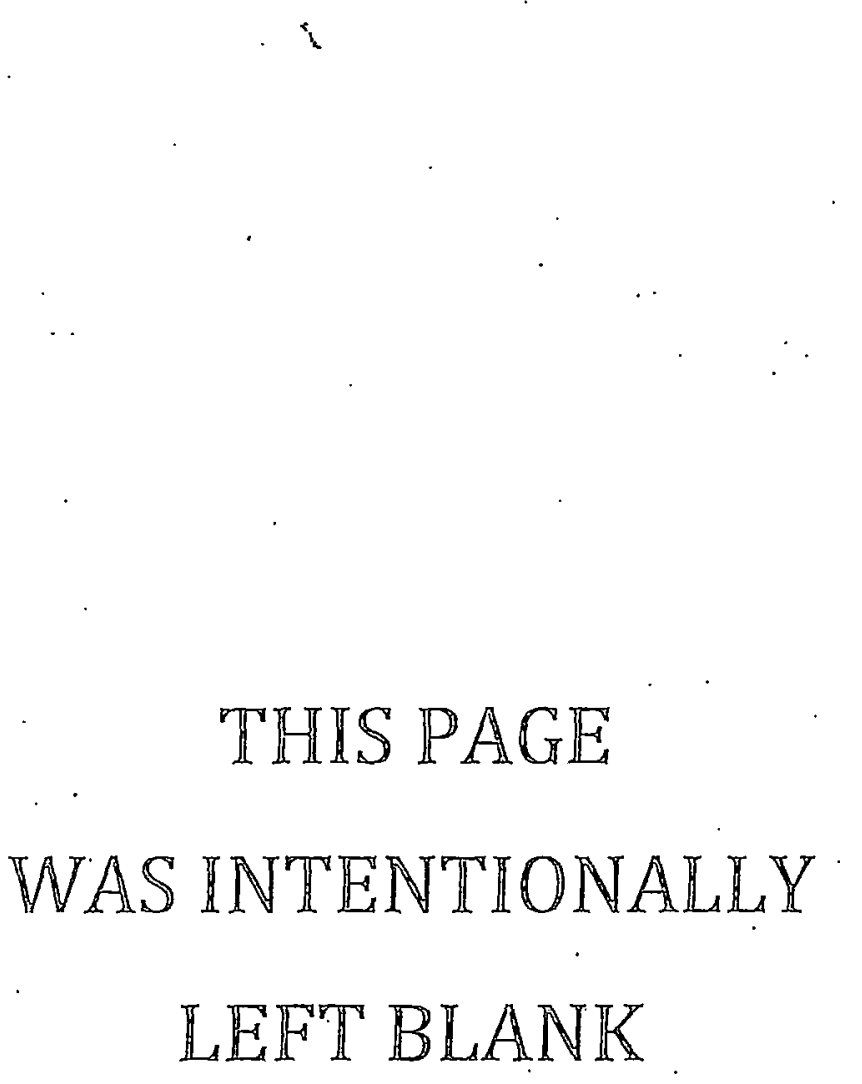


TABLES

ES.1 Machine and plasma parameters for EBT-I, EBT-S, and EBT-II . . . . . . . . . . . 4

2.1 Physical characteristics of EBT-II . . . . . . . . . . . . . . . . . . . 12

3.1 Physical characteristics of EBT-II . . . . . . . . . . . . . . . . . . . 25

3.2 Mirror coil parameters . . . . . . . . . . . . . . . . . . . . . . . 33

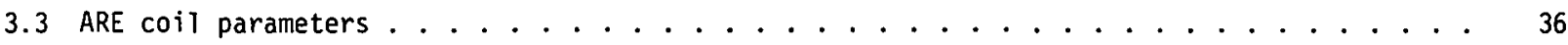

3.4 Summary of resultant magnetic forces . . . . . . . . . . . . . . . . . . . . . . 42

3.5 Vacuum system characteristics . . . . . . . . . . . . . . . . . . . . 49

3.6 Estimated loads ... . . . . . . . . . . . . . . . . . . . 54

3.7 Proposed EBT-II instrumentation . . . . . . . . . . . . . . . . . . . 61

3.8 Cost summary overview . . . . . . . . . . . . . . . . . . . . . . . 78

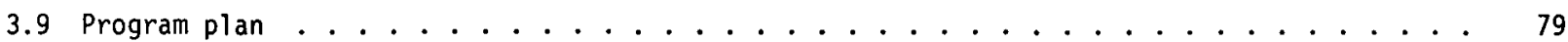

B.1 Physical dimensions and operating parameters . . . . . . . . . . . . . . . . 104

B.2 Product of electron density with carbon ion density and ion lifetime . . . . . . . . 128

B.3 Impurity ion densities in the region of the toroidally confined plasma . . . . . . . 129

E.1 EBT reactor reference parameters . . . . . . . . . . . . . . . . . . . 165

E.2 Expected parameter range for EBT-IT . . . . . . . . . . . . . . . . . . 173 
THIS PAGE

\section{WAS INTENTIONALLY \\ LEFT BLANK}


This document, which was generated at the request of the Department of Energy's 0ffice of Fusion Energy, is an engineering scoping study of EBT-II, the next-generation EBT device. The effort was much too modestly funded to permit a detailed design of EBT-II and should therefore be viewed as a preproposal design guide. Except for an already funded millimeter wavelength, very high power microwave source development program, the study stressed design compatibility with existing technology so as to permit establishing a baseline cost for the machine. The final cost figure is neither lean nor necessarily accurate, but rather is representative of compounded conservatism. 


\section{EXECUTIVE SUMMARY}

The use of ordinary bumpy magnetic field systems for toroidal plasma confinement has been largely ignored by the fusion community because of the susceptibility of such systems to unstable magnetohydrodynamic (MHD) flute modes. However, in the ELMO Bumpy Torus (EBT) at Oak Ridge National Laboratory (ORNL), this problem has been circumvented by the application of electron cyclotron heating (ECH), which, as discovered in mirror research at $0 R N L$, creates high beta, hot electron annuli that modify the toroidal magnetic field in such a way as to prevent MHD instabilities. The primary motivation for EBT confinement research is the fusion reactor goal, which, in this configuration, could provide high beta, steady-state operation in a high aspect ratio, high accessibility reactor embodiment.

The demonstration of stabilization of the toroidally confined plasma by the energetic electron annulus prompted additional studies of the confinement properties of the configuration. Even in the low magnetic field of EBT-I, the stable toroidal plasma attains significant density $\left(\bar{n}_{e}=2 \times 10^{12}\right.$ $\left.\mathrm{cm}^{-3}\right)$, temperature $\left(\mathrm{T}_{\mathrm{e}} \sim 300 \mathrm{eV}, \mathrm{T}_{i} \sim 100 \mathrm{eV}\right)$, and confinement time $\left(\tau_{\mathrm{e}} \sim 3-8 \mathrm{msec}\right)$ to permit favorable comparison with a diffusive neoclassical transport model. With these encouraging results as a baseline, a new experiment, EBT-Scale (EBT-S), is presently under way to provide deeper insight into the scaling of plasma parameters with microwave frequency, magnetic field, and heating power. In addition, the design of a new device, EBT-II, has been initiated. The much higher magnetic field of this device will permit study of plasmas with parameters significantly closer to conceptual reactor conditions and testing of theoretical models over a considerably wider range.

SUMMARY OF PLASMA CONFINEMENT IN EBT

The EBT-I device has experimentaliy provided a proof of principle: demonstration of a smooth transition from the typical MHD unstable bumpy torus regime [when the annulus beta ( $\beta_{\text {annulus }}$ ) is low] into a macrostable hot plasma regime extending over a significant range of operating conditions provided $\beta_{\text {annulus }}$ exceeds the theoretically predicted value for stability. This stabilized toroidal confinement system shares with tokamaks the property of no "mirror-end losses" of the type that lead to large leakage rates and correspondingly low values of the energy gain quotient (Q) for reactor design. However, tokamaks do not behave neoclassically, and they exhibit enhanced anomalous losses that are poorly understood (although these might be colerable in a reactor if it could be made large enough). Furthermore, tokamaks are pulsed devices that are awkward and inflexible to design, build, and operate. The EBT, on the other hand, is a steady-state device with good mechanical access and, at this stage, experimentally correlated neoclassical transport. [It should be noted, however, that there are many active tokamaks and only one EBT, with a second, the Nagoya Bumpy Torus (NBT) in Nagoya, Japan, just starting up.]

Stability calculations have shown that the annuli and the toroidal plasma in EBT serve to stabilize each other and that unstable coupling of the two plasmas does not occur, a fact verified in EBT-I at low beta. Theoretically, ballooning modes in the toroidal core plasma lead to upper limits on the containable amount of core plasma (it is found that values of $\beta_{\text {core }}$ cannot greatly exceed $\beta_{\text {annulus }}$ ); however, the resulting values of $\beta_{\text {core }}$ are high enough to be attractive for reactor designs. Calculations based on MHD theory led to early concern about the ballooning instability of the annulus; however, MHD theory provides an inadequate description of annulus behavior, and a kinetic treatment demonstrates that ballooning modes are stabilized. Also, a number of microinstabilities that could conceivably occur in this system have been studied and are found to be stable for the expected field and plasma gradients. Notable among these are trapped particle modes analogous to those found in tokamaks. 
Theoretical studies have provided no significant evidence of macroscopically unstable behavior of the toroidal plasma in interesting regimes, and the EBT-I experiment has demonstrated nearly quiescent "T-mode" operation with very low density fluctuation levels. A comparison can therefore be made between the experimentally observed plasma parameters (ion and electron temperatures, plasma density, and energy confinement time) and the values predicted by neoclassical transport theory for a stable bumpy torus with ordinary particle collisions and charge exchange as the only loss mechanisms. The results of this comparison are very encouraging: in the T-mode, the EBT-I plasma parameters seem entirely consistent with neoclassical transport predictions. The correspondence is, of course, in a limited range of parameters and has a coarse nature, as dictated by the small size of both experimental and theoretical efforts and by diagnostic limitations associated with operation at low microwave frequency, low magnetic field, and low plasma density.

EBT-I was shut down in November 1977 for conversion to EBT-SCale (EBT-S) by the addition of $200 \mathrm{~kW}$ of $\mathrm{ECH}$ at $28 \mathrm{GHz}$. This machine is presently providing a more definitive test of EBT scaling With microwave trequency at signiticantly higher plasma parameters than were available in EBT-I.

SUMMARY OF THE PROPOSED EBT-II DEVICE

The EBT-II device design represents a dramatic advance for EBT physics and technology and is based on two critical assumptions:

1. Electron and ion transport in EBT-II will be neoclassical, as in the present EBT experiment.

2. The requisite microwave and superconducting technology will be available if sufficient lead time is allowed for development.

The EBT-II machine will be capable of removing the technical restrictions on available parameter space mentioned earlier and will be able to test aggressively the validity of neoclassical scaling because of the increases in basic device parameters: microwave frequency $\left(\omega_{\mu}\right)$ to $120 \mathrm{GHz}$, resonant magnetic field $(B)$ to $4.3 \mathrm{~T}$, and mechanical aspect ratio $(A)$ to about 20 .

The first stage of the experiment, EBT-II Phase 1 , will concentrate on examining the scaling made possible with the device improvements. The higher $\omega_{1}$ and $B$ should allow operation at higher density [since electron plasma frequency $\left(\omega_{\mathrm{pe}}\right) \approx \omega_{\mu}$, plasma density $(n) \approx 2 \times 10^{14} \mathrm{~cm}^{-3}$ ] where, as an added benetit, plasma diagnostics are more reliable. In addition, the larger aspect ratio should improve the neoclassical confinement time (which scales as $A^{2}$ ) by an order of magnitude. An additional

'Readers of previous EBT discussions should note that a convention has been established for defining the mechanical and effective aspect ratios of the torus. The mechanical aspect ratio (A) is defined as $\mathrm{K}_{T} / \bar{r}_{c}$, where $\mathrm{K}_{T}$ is the major radius of the torus and $r_{c}$ is the mean minor radius of the toroidal magnetic field coils. The effective aspect ratio $\left(A_{\text {eff }}\right)$ with ARE coils is given by

$A_{\text {eff }}=\frac{R_{T}}{R_{C}} f_{A R E} \cong A f_{A R E}$,

where $f_{A R E}$ is the ARE coil enhancement factor and $R_{C}$ is the radius of curvature of the magnetic field in a sector midplane. In EBT-II, the mechanical aspect ratio will be 20, and the effective aspect ratio may be as high as 50 with the maximum ARE coil current (or as low as 10 with the ability to reverse the polarity of ARE coil current). 
feature designed into the device in Phase 1 is the ability to control flexibly and to increase the effective aspect ratio with supplementary toroidal aspect ratio enhancement (ARE) coils and thereby to push the scaling test even further. The current design calls for ARE factors of about two which should increase the neoclassically calculated lifetime greatly (see Sect. 2). Under optimum conditions, EBT-II Phase 1 may yield confinement time $(\tau)$ of $\sim 0.2 \mathrm{sec}$, temperature $(T)$ of $\sim 5 \mathrm{keV}$, and $n_{\tau}$ of $\sim 10^{13}$ $\mathrm{sec} / \mathrm{cm}^{3}$.

Phase 2 will center on the addition of 1 MW of neutral beam heating to heat ions as well as electrons directly, providing control over the ambipolar potential. With the added heat and particle source allowing control of the electric field, one can predict neoclassically an increase by at least a factor of two in energy lifetime at increased plasma density. It is possible that Phase 2 of EBT-II could demonstrate near-breakeven conditions in hydrogen with $\beta \sim 10 \%, \tau \sim 1 \sec , \mathrm{n} \sim 10^{14} \mathrm{~cm}^{-3}$, $T \sim 5-10 \mathrm{keV}$, and $n_{\tau} \sim 10^{14} \mathrm{sec} / \mathrm{cm}^{3}$.

The parameters of EBT-I, EBT-S, and EBT-II are listed in Table ES.l. Some of the EBT-II parameters may be revised slightly as the detailed engineering design progresses.

The details of the theoretical effort and the experimental observations are found in Sect. 1 and in Appendixes $A$ (theoretical effort) and $B$ (experimental observations). Section 2 presents the proposed EBT-II experiment, and Sect. 3 is the engineering design description of the EBT-II machine. Section 4 delineates risks and exposure hazards; Sect. 5 is a brief statement of environmental impact. Detailed discussions of EBT-II magnetics design are found in Appendix C. Appendix D gives details of neutral beam heating for EBT-II, and the relevance of the EBT-II experiment to an EBT reactor is discussed in Appendix $E$. 
Table ES 1. Mazhine and plasma parameters for EBT-I, EBT-S, and E3T-II

\section{MACHINE}

MAGNETIC FIELD (MIDPLANE, MIRROR) MAGNETIC FIELD FOWER

TOROIOAL FIELD COILS

ARE COILS

TORUS VOLUME

MANOR RADIUS

COIL MEAN F:ADIUS

ASPECT RATIO

MICROWAVE POWER, CONTINUOUS WAVE

(CW)

BULK HEATING

PROFILE HEATING

NEUTRAL BEAM HEATING (CW)

\section{HOT ELECTRON ANNULUS}

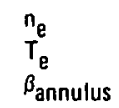

\section{TOROIDAL PLASMA}

$\mathrm{T}_{\mathrm{e}}$

$T_{i}$

MIOPLANE MINOR RADIUS

EFFECTIVE ASPECT RATIO

VOLUME

\begin{tabular}{|c|c|c|c|c|}
\hline $\begin{array}{c}\frac{\text { PAST }}{\text { EBT.I }} \\
\text { EXPERIIAEVTS CONCLUDEO } \\
\text { NOVEMEER 19:7 } \\
\text { MEEASURED) }\end{array}$ & $\begin{array}{l}\frac{\text { PRESENT }}{\text { EBT.S }} \\
\text { IEXPERIMENTS BEGIN } \\
\text { SUMMER 1978 } \\
\text { (ANTICIPATED) }\end{array}$ & \multicolumn{3}{|c|}{$\begin{array}{l}\frac{\text { FUTURE }}{\text { EBT-II }} \\
\text { EXPERIMENTS BEGIN } \\
\text { POSSIBLY FY } 1982 \\
\text { (ANTICIPATED) }\end{array}$} \\
\hline $0.45 \mathrm{~T}, 0 \subseteq \mathrm{T}$ & $0.7 \mathrm{~T}, 1.4 \mathrm{~T}$ & \multicolumn{3}{|c|}{$3.0 \mathrm{~T}, 6.0 \mathrm{~T}$} \\
\hline $\begin{array}{l}7.5 \text { UW } \\
\text { NONE } \\
135 C \text { liters } \\
150 \mathrm{~cm} \\
18.8 \mathrm{~cm} \\
8: 1\end{array}$ & $\begin{array}{l}12 \mathrm{MW} \\
1350 \text { liters } \\
150 \mathrm{~cm} \\
18.8 \mathrm{~cm} \\
8: 1\end{array}$ & \multicolumn{3}{|c|}{$\begin{array}{l}1.3 \mathrm{~kW} \text { (REFRIGERATION) } \\
15 \mathrm{MW} \\
5500 \text { liters } \\
520 \mathrm{~cm} \\
28 \mathrm{~cm} \\
\approx 20: 1\end{array}$} \\
\hline $\begin{array}{l}60 \mathrm{kN}, 18 \mathrm{GHz} \\
30 \mathrm{kN}, 106 \mathrm{GHz} \\
\text { NONE }\end{array}$ & $\begin{array}{l}200 \mathrm{~kW}, 28 \mathrm{GHz} \\
60 \mathrm{~kW}, 18 \mathrm{GHz} \\
\text { NONE }\end{array}$ & \multicolumn{3}{|c|}{$\begin{array}{l}1.6 \mathrm{MW}, 120 \mathrm{GHz} \\
800 \mathrm{~kW}, 70.90 \mathrm{GHz} \\
1 \mathrm{MW} \text { AT } 20 \mathrm{kV}\end{array}$} \\
\hline $\begin{array}{l}2.5 \times 10^{11} \mathrm{~cm}^{-3} \\
100-300 \mathrm{keV} \\
10-43 \%\end{array}$ & $\begin{array}{l}2.5 \times 10^{11} \mathrm{~cm}^{-3} \\
100.500 \mathrm{keV} \\
10-40 \%\end{array}$ & \multicolumn{3}{|c|}{$\begin{array}{l}1.6 \times 10^{12} \mathrm{~cm}^{-3} \\
500.2000 \mathrm{keV} \\
10.50 \%\end{array}$} \\
\hline & & $\begin{array}{c}\text { PHASE } 1 \\
\text { WITHOUT ARE }\end{array}$ & $\begin{array}{c}\text { PHASE } 1 \\
\text { WITH ARE }\end{array}$ & PHASE 2 \\
\hline $\begin{array}{l}1.4 \times 10^{12} \mathrm{~cm}^{-3} \\
150-300 \mathrm{el} \\
70-150 \mathrm{eV} \\
0.2 \cdot 6.6 \%\end{array}$ & $\begin{array}{l}2.6 \times 10^{12} \mathrm{~cm}^{-3} \\
300-800 \supseteq \mathrm{V} \\
100-200 \supseteq \mathrm{V} \\
-0.5 \%\end{array}$ & $\begin{array}{l}\sim 5 \times 10^{13} \mathrm{~cm}^{-3} \\
3 \mathrm{keV} \\
1 \mathrm{keV} \\
\leq 1 \%\end{array}$ & $\begin{array}{l}\sim 5 \times 10^{13} \mathrm{~cm}^{-3} \\
3.8 \mathrm{keV} \\
3.8 \mathrm{keV} \\
\geq 1 \%\end{array}$ & $\begin{array}{l}\sim 10^{14} \mathrm{~cm}^{-3} \\
5 \cdot 10 \mathrm{keV} \\
5 \cdot 10 \mathrm{keV} \\
\sim 10 \%\end{array}$ \\
\hline $\begin{array}{l}10 \mathrm{~cm} \\
8: 1 \\
400 \text { liters } \\
5 \times 10^{10} \mathrm{sec} \mathrm{cm}^{-3}\end{array}$ & $\begin{array}{l}10 \mathrm{~cm} \\
8: 1 \\
400 \text { liters } \\
\sim 10^{11} \mathrm{sec} \mathrm{cm}^{-3}\end{array}$ & $\begin{array}{l}\leqslant 14 \mathrm{~cm} \\
\approx 20: 1 \\
\leqslant 1400 \mathrm{li} \cdot \mathrm{e}: \\
\sim 10^{12} \mathrm{sec} \mathrm{cm}^{-3}\end{array}$ & $\begin{array}{l}\leqslant 17 \mathrm{~cm} \\
\approx 40: 1 \\
\leqslant 2000 \text { iters } \\
\sim 10^{13} \mathrm{sec} \mathrm{cm}^{-3}\end{array}$ & $\begin{array}{l}\leqslant 17 \mathrm{~cm} \\
=40: 1 \\
\leqslant 2000 \text { liters } \\
\sim 10^{16} \mathrm{sec} \mathrm{cm}^{-3}\end{array}$ \\
\hline
\end{tabular}




\section{THE PRESENT STATUS OF EBT}

The ELMO Bumpy Torus (EBT) is a specific bumpy torus device designed to circumvent the magnetohydrodynamic (MHD) stability problem by using the "minimum-B" properties produced by annular, high beta, hot electron plasmas formed by microwave heating in open-ended magnetic traps. Figure 1.1 illustrates the geometry of this confinement system. The shaded regions inside the cutaway cavities are the circulating toroidal plasma and the annular, mirror-confined, relativistic electron plasmas in each torus bump.

Results from experiments carried out in EBT during the past few years have unambiguously supported the basic EBT premise: azimuthal plasma currents produced by the high beta, hot electron annuli cause changes in the magnetic field gradients which provide macrostable plasma confinement in a bumpy torus. In addition to achieving this primary objective, the EBT experimental program has generated a considerable amount of important plasma parameter information, including observation of high energy, well confined toroidal plasmas; high plasma purity; densities approaching microwave cutoff; increased plasma temperature with increased microwave power; and no major degradation of confinement caused by convective cells or field errors at reasonable levels of construction difficulty.

Although the present EBT is only a first-generation device and, more notably, the first device of $i$ ts kind, the existing experimental and theoretical evidence indicates that the EBT approach can be extended into reactor regimes to confine steady-state, high beta (toroidal) plasmas. If this extension can be demonstrated in subsequent experimental devices, then EBT offers an attractive alternative to the tokamak and magnetic mirror approaches to controlled thermonuclear fusion.

In November 1977 experimental work on EBT-I was concluded and conversion of EBT-I to EBT-SCale (EBT-S) began. The remainder of this section summarizes the present status of experimental and theoretical work on EBT.

\subsection{EXPERIMENTAL RESULTS OF EBT-I}

Detailed research on plasma confinement and heating has been carried out in EBT over the past several years with the following experimental conclusions.

1. Three distinct, reproducible mones of operation, the $C_{-}, T_{-}$, and M-modes, are observed as the ambient gas pressure is gradually decreased. The confinement, heating, and gross stability observed in the T-mode (at intermediate gas pressures) are quite similar to the anticipated behavior described in the original expositions of the EBT concept.

2. The hot electron annuli, with values of beta ranging to 0.5 , are stable toward filuctuations if the ambient gas pressure exceeds a critical value which depends on the microwave power.

3. The EBT experiments have demonstrated the importance of the reflux of gas at the cavity wall in determining the source of particles required to maintain the plasma in steady state. The dominant source of fresh ion-electron pairs is energetic neutral hydrogen recirculated at the wall. Significant sputtering of the aluminum walls is observed under some conditions, but impurity densities in the coroidal plasila have so far remained at tolerably low values $\left(n_{1} / n_{e} \sim 10^{-4}\right)$.

4. In the T-mode, at electron densities of $\mathrm{n}_{\mathrm{e}} \approx 2 \times 10^{12} \mathrm{~cm}^{-3}$, the bulk fluctuation levels are approximately at thermal levels with ion temperature around $100 \mathrm{eV}$ and mean electron temperatures ranging up to $500 \mathrm{eV}$.

5. The observed scaling of the device shows $T_{e}$ increasing with microwave power and density depending on neutral feed rate - in qualitative agreement with simple models. The measured neutral hydrogen density profiles, the temperatures $\left(T_{e}, T_{i}\right.$, and $\left.T_{0}\right)$, and other critical parameters qualitatively agree with the theoretical models which employ neoclassical diffusive transport rates. 
ORNL / DWG 73-5045R4

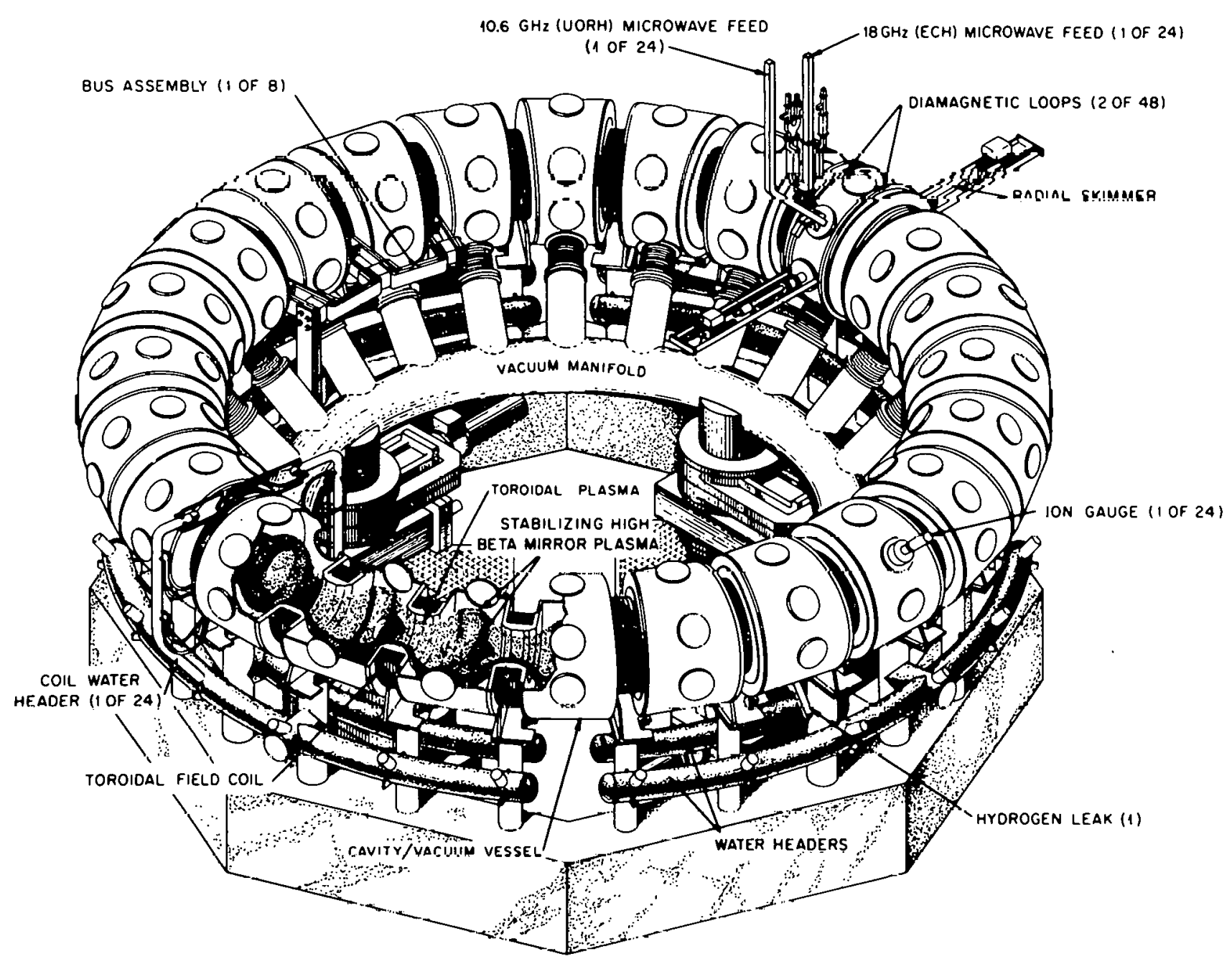

F1y. 1.1. Schematic layour of tíbl-11. 
6. Measurements of the ambipolar potential profile also corroborate the model conclusions.

7. Energy confinement times for the bulk plasma are hundreds of Bohm times and are about what is expected from neoclassical transport.

\subsubsection{Electron Temperature}

Bremsstrahlung measurements of the electron temperature have confirmed Thomson scattering measurements made with $30 \mathrm{~kW}$ at $18 \mathrm{GHz}\left(T_{e} \sim 150 \mathrm{eV}\right)$. Further, it was found that the energy deposition in the toroidal plasma increases with microwave power in the T-mode. Electron temperatures up to 500 eV were observed by bremsstrahlung measurements with $60 \mathrm{~kW}$ at $18 \mathrm{GHz}$ in T-mode plasmas. Studies of the high energy annulus with a multiple array of magnetic loops for determining the near-field distribution of the diamagnetic currents have confirmed the limited axial size and width of the annuli. The electron energy in the annulus is approximately $100 \mathrm{keV}$ with $\mathrm{n}=5 \times 10^{11} \mathrm{~cm}^{-3}$.

\subsubsection{Ion Energy}

Measurements of the ion energy distribution using charge exchange neutral analyzers were carried out over a wide range of operating conditions. The ion energies in a hydrogen plasma have been observed to increase with microwave power up to $T_{i} \sim 150 \mathrm{eV}$. Higher energy groups of ions have been noted, but these normally constitute only a small percentage of the toroidally trapped ion population. Repeated observations under $\mathrm{C}$-mode conditions confirmed the absence of observable concentrations of warm ion groups when the plasma is unstable. An energetic ion velocity filter was used in tandem with the ion energy analyzer to show that the charge exchanging particles have no perceptible energetic impurity population. Radial ion temperature profiles were measured and seen to be practically constant in radius out to $215 \mathrm{~cm}$ throughout the $T$-mode.

\section{1 .3 spectroscopy}

A single uv spectrometer used with two collimated scanning mirrors installed on EBT provided spatially resolved, absolute intensity measurements of the carbon and aluminum ion impurity distributions and the neutral hydrogen distribution. The measurements clearly show that some impurity ions are present in the toroidal plasma, although, for all species, the density is observed to be lower than that seen in the surface plasma. The best estimate of the ratio of carbon to electron density in the toroidal plasma, $n(c) / n_{e} \leqslant 1 \times 10^{-4}$, indicates that the surface plasma provides effective shielding against incoming impurities. In addition, the neutral hydrogen light distribution was unfolded into a surface distribution concentric with and outside the electron annulus and the toroidal core plasma. The result of the analysis of the optical radiation from the toroidally trapped region is consistent with ionization of incoming neutrals and consequent reduction of neutral density at the plasma core, as estimated in the one-dimensional transport model.

\subsubsection{Plasma Potential Measurement}

A heavy ion beam probe system was adapted to steady-state EBT operation and was used to determine the plasma potentials and their gradients. Measurements of the potentials in the T-mode with a Rb beam have always shown a large positive peak ( $200 \mathrm{~V}$ ) at the electron annulus location, with a negative (inward pointing) electric field. The potential at the axis could be either positive or negative with respect to the walls. Under conditions in which the toroidal current was minimized, the potential profiles were nearly symmetrical over the minor cross section of the plasma. 


\subsubsection{Fluctuation Studies}

Using wide band probes, fluctuation studies were carried out from $500 \mathrm{~Hz}$ to $40 \mathrm{GHz}$. The earlier observations confirmed that low frequency ( $f<100 \mathrm{kHz}$ ) fluctuations predominant in $\mathrm{C}$-mode are suppressed in T-mode. Detailed spatial measurements showed the stability of the toroidal core plasma when the annuli were established, even though low frequency instabilities persisted outside the radius of the annulus. Studies of higher frequency oscillations were also undertaken and fluctuations at $\omega_{c e}$ were noted at the location of the energetic annulus.

\subsection{THEORETICAL RESULTS AND COMPARISON WITH EXPERIMENTS}

The essence of the theoretical work done on EBT is summarized in this section; more detail is found in Appendix $A$.

\subsubsection{Equilibria}

Codes have been developed to demonstrate the existence of full three-dimensional equilibria in bumpy tori with finite aspect ratio and with diamagnetic currents carried by hot electron annuli. These codes are used to determine the sensitivity of particle trajectories and confinement to the parameters of the annuli, the external field shaping currents, and the ambipolar (radial) electric potentials that are observed in the experiments.

\subsubsection{Stability}

Calculations made with the guiding center fluid model have shown that the hot electron annulus in an EBT can provide the requisite stabilization of the toroidal or core plasma. The flute modes which affect a conventional-bumpy torus are stabilized by the effects of the local magnetic well formed near the hot electron annulus when the value of Bannulus exceeds a minimum value. This critical minimum value of $\beta_{\text {annulus }}$ depends somewhat on details of the assumed annulus configuration, but lies in the range nf $5-10 \%$.

Stability of the core plasma leads to a restriction on $\beta_{\text {core }}$ to levels less than $B_{\text {annulus }}$ times

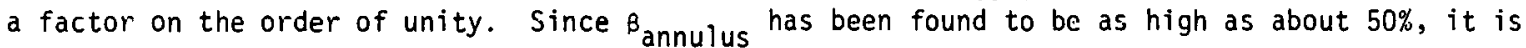

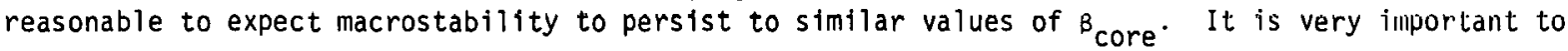
test this point experimentally; EBT-II Phase 2 (see Sect. 2) should give an increase of about two orders of magnitude in $\beta_{\text {core }}$ if the observed neoclassical behavior persists. The EBT-II experiment should be able to provide the improved understanding that would justify a full-scale test of operation at the beta levels required for reactor operation (see Sect. 2.3).

The stability considerations for the hot electron annuli are dominated by the experimental observation of macrostability. A theoretical treatment that includes velocity space effects in the annulus shows that the stability can be explained as a result of the presence of the high density core plasma extending out to the region of the annulus.

An initial survey has been made of possible microinstabilities which might affect EBT-I, -II, or reactor operation ( $i t$ is encouraging to note that the change in collisionality between EBT-I and the reactor regime is only about an order of magnitude). The trapped particle modes, conjectured to have a large effect on tokamak operation, have been analyzed for EBT and are found to have stabijity conditions similar to MHD flute and ballooning modes. 
The effect of magnetic field errors on EBT stability and confinement has been analyzed experimentally and theoretically. A model has been advanced in which the parallel currents resulting from field errors lead to MHD instabilities when a threshold value is exceeded. This agrees qualitatively with the observed behavior, although detailed studies which can establish unambiguous identification of the modes have not been completed.

\subsubsection{Transport Coefficients}

Procedures have been developed for consistent calculation of the particle transport coefficients for EBT, taking into account the observed electrostatic radial potential distribution. A very important result of this is an understanding of the large changes in transport coefficients which result from electric field modifications of the particle orbits. These results are used as input to the transport calculations described in Sect. 1.2.4.

\subsubsection{Transport Calculations}

Procedures and codes at three levels of sophistication are available for analyzing the performance of EBT systems. First, a point model is used to study very global questions, such as scaling to and optimization of reactor systems.

Second, a radially resolved one-dimensional code is used for detailed comparison of experimental data with predictions using neoclassical transport coefficients, self-consistent radial electric fields, and neutral particle distributions from the Oak Ridge Tokamak Transport Code. The code includes most of the applicable physics and can generate, over a limited range of parameters, results that agree qualitatively with observations.

Finally, a kinetic treatment model is used to make more detailed studies of how the radial electric field is formed and what its controlling factors are. Since it became possible to obtain experimental radial potential profiles, there have been marked advances in the understanding of the electric field and its effects on confinement.

\subsubsection{Electron Heating and Microwave Propagation}

Analysis and code development are under way with the ultimate goal of predictive calculation of the plasma heating process, taking into account the propagation effects, polarization effects, and coupling to parasitics through parametric or other processes. 
THIS PAGE

\section{WAS INTENTIONALLY \\ LEFT BLANK}




\section{THE PROPOSED EBT-II EXPERIMENT}

The EBT confinement and heating approach to magnetic fusion has unambiguously demonstrated, in the EBT-I experiment, that mirror-confined relativistic electron annuli modify the characteristically unstable magnetic field gradients to allow macrostable operation of a steady-state bumpy torus plasma. The confinement in T-mode operation is. consistent with collisionless neoclassical transport ${ }^{\star}$ in the reactor plasma regime. However, complete justification of the EBT idea as a viable reactor concept. requires a test of the principle in a regime which contains plasma parameters closer to the reactor regime than those obtainable so far. The existing program plan for. EBT provides for a sequence of experiments deșigned to test definitively this confinement concept through the reactor plasma regime.

The very encouraging results from the EBT-I experiments showed that a relatively clean plasma was confined in a macrostable steady state with an energy replacement time consistent with the predictions of classical theory. However, at the low density and modest temperature characteristic of EBT-I, the classical losses were large enough to mask a nontrivial amount of turbulant loss. Further, the modest parameter range accessible in the experiment could not be considered sufficientiy broad to establish the credibility of theoretical extrapolation to reactor-like parameters. Thus, in this sense, EBT-I cannot be considered to have been a definitive experiment. .

A new experiment, EBT-Scale (EBT-S), is presently providing important additions to the EBT data base. EBT-S is a modification of the EBT-I facility and is designed to be a more definitive test of transport scaling. It is aimed at demonstrating the anticipated scaling of density with microwave frequency, thereby obtaining plasma parameters $\left(T_{e}, T_{i}, n_{e}\right)$ significantiy higher than those of EBT-I.

A later stage of EBT-S, EBT-S/A, will employ, in addition to the basic toroidal field coils, relatively low current, field shaping coils or aspect ratio enhancement (ARE) coils, which will permit convenient experimental variation of the effective aspect ratio. In the existing theory of the neoclassical collisionless regime, the plasma lifetime is roughly proportional to the square of the aspect ratio, so the ARE coils, used in both bucking and current aiding senses, will permit a wide variation of confinement geometry. Detailed examination of fluctuation spectra as the plasma regime is varied should lead to unambiguous identification of plasma turbulent activity and its influence on plasma containment.

The scaling of EBT-II is based on the results of EBT-I and those anticipated frum EBT-5 and EBT-S/A. However, EBT-II is not only a significant plasma parameter extrapolation but is also a major scaleup in device size, magnetic field, microwave heating frequency, and power over EBT-S/A. The ma in physical characteristics of EBT-II are summarized in Table 2.1 and the design details are found in Sect. 3. The basic device is essentially a toroidal vacuum vessel, consisting of 48 superconducting magnet dewars alternating with 48 wedge-shaped cavity sections, in which a steady-state plasma is contained by the magnetic field generated by the superconducting magnets and stabilized by the hot electron annuli. Microwave sources at two or more different frequencies (70-90 GHz and $120 \mathrm{GH}$ ) are needed for separate control of the hot electron annuli and the toroidal core plasma. The experiment is designed to proceed in two phases.

In Phase 1 of EBT-II, the ARE coils and electron cyclotron heating (ECH) at frequencies up to $120 \mathrm{GH} 7$ will be used to confirm density scaling with magnetic field and to improve plasma parameters to levels substantially above any anticipated low beta instability thresholds, thereby reaching

\footnotetext{
*By "neoclassical transport" it is meant that losses occur by binary collisions. This use of "neoclassical" includes geometric effects, thus encompassing the terms "neoclassical" and "classical" as they appear in the technical literature.
} 
significant values of bulk beta in the toroidal plasma. The anticipated $n_{\tau}$ in this regime is $\sim 10^{13}$ $\mathrm{sec} / \mathrm{cm}^{3}$, as scaled from the limited experimental base now available.

In Phase 2 of EBT-II, auxiliary neutral beam injection heating will be employed. This added ion heating power would make EBT-II parameters comparable with those claimed for existing tokamaks, given favorable results in Phase 1 and agreement with the more pessimistic versions of current neoclassical transport. More favorable neoclassical scaling exists in which losses are dominated by ions rather than electrons. Some optimism exists that this regime can be reached when auxiliary ion heating rather than pure electron heating is employed, in which case breakeven plasma parameters would be obtained. (This would require taking full advantage of the improved confinement resulting from control of the plasma potential by the neutral beams or from appropriate adjustment of the potential by the plasma itself.)

Table 2.1. Physical characteristics of FRT-TT

Torus

$\begin{array}{ll}\text { Number of cavities } & 48 \\ \text { Number of mirror coils (superconducting) } & 48 \\ \text { Coil current density } & 6650 \mathrm{~A} / \mathrm{cm}^{2} \\ \text { Cavity volume } & 4.5 \mathrm{~m}^{3} \\ \text { Coil throat volume } & 0.97 \mathrm{~m}^{3} \\ \text { Total vacuum volume } & 5.5 \mathrm{~m}^{3} \\ \text { Torus major radius } & 5.2 \mathrm{~m}\end{array}$

Microwave system

Total input power

Number of tubes (gyrotrons)

Microwave frequency

Resonant heating

Prnfile heating

Resnnant. magnetic field

$1.6 \mathrm{MW}$

$800 \mathrm{~kW}$

8

$170 \mathrm{GHz} \quad 70-90 \mathrm{GHz}$

$1.3 \mathrm{~T} \quad 2.5-3.2 \mathrm{~T}$

Aspect ratio enhancement (ARE)

Number of coils (normal copper) 96

Coil current density

$1505 \mathrm{~A} / \mathrm{cmI}^{2}$

Kange of effective aspect ratio $10-50$

Neutral beam injection

Type of injection Perpendicular (vertical)

Energy of neutral beam $20 \mathrm{keV}$

Total input power $1 \mathrm{MW}$

Initial plasma parameter scaling studies can be carried out for approximately one year, the period scheduled for Phase 1 operations. The installation of neutral bcam injectors for Phase 2 experiments will follow the completion of Phase 1 . Once this is done, an initial plasma density of about $5 \times 10^{13} \mathrm{~cm}^{-3}$ obtained with ECH only will permit trapping a significant portion of the perpendicularly injected 20-keV neutral beam (see Appendix D). Preliminary calculations indicate that 
absorption of $\sim 300 \mathrm{~kW}$ of beam power in the toroidal plasma will raise the ion density to $\sim 10^{14} \mathrm{~cm}^{-3}$ with confinement times approaching $1 \mathrm{sec}$.

In Sect. 2.1, a discussion of the parameter decision chain for the EBT-II design is presented. In Sect. 2.2, the critical questions in the EBT program which will be addressed through the EBT-II device are discussed.

\subsection{DECISION CHAIN FOR SPECIFIC EBT-II PARAMETERS}

The reference design discussed in this section does not represent a fully optimized set of parameters; rather, it demonstrates an attractive possibility for the resolution of the critical EBT questions at relatively reasonable cost. The required levels of technology are already available or are the end product of existing industrial development contracts. The following discussions establish the technology control factors in the existing design study, give some indication of their system sensitivities, and provide the basis for the required final design tradeoff studies. Studies aimed at minimizing cost and maximizing effectiveness will proceed as the next step toward a detailed engineering design for the EBT-İI device.

\section{1 .1 Device Cost}

The device cost goal was originally set at $\$ 30$ million following discussions of budget projections through FY 1981 with the Department of Energy's Division of Magnetic Fusion Energy (DOE-DMFE). However, this goal was set for a device without ARE, neutral beam heating, or the prospect of breakeven. The more aggressive conceptual design described here is listed at approximately $\$ 70$ million, including a realistic $25 \%$ for contingencies (see Sect. 3.2 ).

\subsubsection{Fusion Plasma Relevance}

The magnetic field level, magnetic field configuration, and torus volume of EBT-II were chosen to permit collisionless neoclassical plasma confinement in a reactor-relevant density regime, i.e., $10^{13} \leq n_{e}: 10^{14}$. The remaining gross parameters of the device then follow readily. Target electron and ion temperatures were selected to place EBT-II Phase I near the projected reactor collisionality range; i.e., $T_{i}>0.5 \mathrm{keV}$ and $T_{e}>2 \mathrm{keV}$, with unenhanced aspect ratio $A \approx 20$, and neoclassical confinement times of roughly $0.2 \mathrm{sec}$, yielding an $n \tau$ of $210^{13} \mathrm{~cm}^{-3} \mathrm{sec}$. A more detailed discussion of EBT-II plasma parameter scaling is found in sect. 2.2.2.

\subsubsection{Near-Term Technological Feasibility}

The principal technological constraints are imposed by superconducting magnet and microwave source limitations. Because the conventional application of ECH requires that the electron cyclotron frequency $\left(\omega_{\mathrm{ce}}\right)$ he greater than the elestron plasma frequency $\left(\omega_{\mathrm{pe}}\right)$, the plasma density goal of $\mathrm{n} \geqslant 10^{14} \mathrm{~cm}^{-3}$ determines a minimum cutoff density of $1.5 \times 10^{14} \mathrm{~cm}^{-3}$ and a bulk microwave heating frequency of $120 \mathrm{GH}$. The probability of realizing 200-k.W r.w microwave sources at $120 \mathrm{GHz}$ was deemed much greater than that for higher frequency sources. This frequency choice also determines the ECH resonant magnetic field value $\left(B_{r}\right)$ of $4.3 \mathrm{~T}$. Since a mirror ratio $(M)$ of at least $2: 1$ on axis is required for relativistic annulus formation, this geometric mean resonant field value $\left(B_{r}=B_{\text {max }} / \sqrt{M}\right)$ 
results in a maximum field strength of 7.5-8.0 $\mathrm{T}$ in the current winding, thereby permitting the use of niobium-titanium (NBTi) superconducting toroidal field magnets. At this field (plasma density) level, a significant density scaling test is possible; reducing the field does not lead to sufficient reduction in cost to justify the limitations on plasma parameters and range of possible scaling tests. The choice of major radius, number of sectors, and aspect ratio enhancement magnet design within the constraints described above is then made so as to maximize particle confinement. Details of the magnetics design studies are given in Appendix $C$.

The development of very high power $\mathrm{Cw}$ microwave heating sources feasible for frequency scaling to $120 \mathrm{GHz}$ was subcontracted in 1975 to Varian Associates, Palo Alto, California. Sources from this promising program based on cyclotron resonant maser amplifiers (gyroklystrons) and oscillators (gyrotrons) are expected to make a good time mesh with the EBT-II machine construction program. These sources are expected to be $200-\mathrm{kW} \mathrm{cW}$ modules and to cost about $\$ 4 / W$ installed. The magnetic field geometry was chosen so as to supply power to the plasma at the required 1 W/cm ${ }^{3}$ with $1.6-M W$ input., thus satisfying a reasonable microwave cost allocation. A smaller magnetic vnlums wnuld rodura the cost, but the inward protrusion of the superconducting coil x-ray shield (see Sect. 3.1.3) would then grcatly interfere with collisionless tenrnital particle sirculation. The EBT II mierowave heatilly syslem ls described in Sect. 3.1.5.

It should be mentioned that the microwave tube development program for EBT-II is prcsently proceeding at an encouraging pace and upon its culmination is expected to provide the tube modules required for the ECH power distribution system. Operation of this system would be a scale demonstration of much of the heating technology for the EBT reactor.

\subsection{CRITICAL QUESTIONS}

The $t B I-1 \perp$ experiment will address a number of critical questions in the technological and sclentitic domains. The demonstration of requisite technology, the investigation of sraling laws, and the study of confinement at higher values of beta can be achieved in FRT-II.

\subsubsection{Deflunstration of Kequisite Technology}

\subsubsection{Heating}

Two of the more important technological questions which can be resolved in this experiment are the effectiveness of large-scale microwave ECH and neutral beam heating of a high density EBT plasma while maintaining control of the plasma particle feed.

Because the plasma parameters of EBT-II should approach thnse nf an EBT reastor, EBT II should provide an adequate test of both environmental compatibility and coupling efficiency of ECH. It should also permit an assessment of reliability, tractability, and operating costs of a large array of high power millimeter gyrntron sources.

\subsubsection{Equilibrium control}

The ambipolar electric field profile, which greatly influences EBT particle diffusion rates, can be modifled by all independent fon heat source. Neutral beam heating, which will be employed in Phase 2 (see Appendix 0 for details), will be used for potential profile control, plasma heating, and particle replenishment to obtain an optimized equilibrium. 


\subsubsection{Plasma feed}

Plasma particle replenishment is by no means the least significant use of neutral beams, although it is certainly their least studied function. The two principal particle sources for the EBT-II plasma will be the direct feed of energetic beam neutrals and the surface feed of cold gas from the neutral gas halo outside the hot electron rings. The cold gas halo, required for ring plasma stability, poses few problems other than those related to efficiency, but the bulk plasma replenishment of particles may require detailed spatial injection.

\subsubsection{Scaling Criteria for EBT-II}

The quiet T-mode of operation in EBT-I suggests that the loss rates in EBT are governed by classical and neoclassical processes. The theory of neoclassical transport in EBT is rather complicated, in part because of the complexities introduced by the ambipolar electric field. Thus, the theory of neoclassical transport has undergone several evolutions. Nonetheless, certain features of the theory have been invariant since the inception of the EBT program. By using these most clear-cut features of the theory, it is possible to develop semiempirical scaling criteria which can be used to predict the plasma parameters for EBT-II. Semiempirical scaling limitations imposed on plasma density by microwave cutoff are discussed first, followed by a discussion of some neoclassical scaling criteria.

Experience with several machines heated with ECH has shown that plasma heating is effective at densities approaching microwave cutoff. This implies that the upper limit on density, $n$, scales as the square of the magnetic field. Since there is a five-fold increase in the magnetic field between EBT-I and EBT-II, and since $n \cong 2 \times 10^{12} \mathrm{~cm}^{-3}$ in EBT-I, then the density of EBT-II Phase 1 should be approximately $5 \times 10^{13} \mathrm{~cm}^{-3}$. If neutral beam heating is used, then the microwave cutoff restriction for the central core plasma can be slightly reduced, and the density can be somewhat larger (i.e., $\left.\mathrm{n} \leqslant 10^{14} \mathrm{~cm}^{-3}\right)$.

Consideration is now given to those aspects of semiempirical scaling which are associated with neoclassical transport. Recent analysis of data from EBT-I shows remarkable agreement with one of the basic elements of neoclassical transport theory - the electron heat conduction coefficient. Even more recent is the derivation of improved expressions for the ion transport coefficients. The formal solutions are sufficiently complicated that implementation of these transport coefficients is not complete at this time. Instead of using premature approximations for these transport coefficients, semiempirical scaling has been chosen for predicting the plasma parameters in EBT-II. This limits the predictions to EBT-II Phase 1 operation, which does not employ neutraj beam injection. A yuditative discussion of the probable effects of neutral beam injection is included at the end of this discussion.

In EBT-I there was a minimum observed value of collisionality. In order to make conservative estimates for EBT-II, the collisionality can be assumed to be constant. The electron collisionality scales as $n B / T_{e}^{5 / 2}$, and $n$ scales as $B^{2}$, so that the collisionality scales as $B^{3} / T_{e}^{5 / 2}$. (Implicit in this scaling is that $e_{\phi} / T_{e}$ is constant.) Assuming constant collisionality, a factor of five increase in B leads to a factor of seven increase in $T_{e}$. For EBT-I, $T_{e}$ lies in the range of $300-600 \mathrm{eV}$, so that for EBT-II, $T_{e}$ lies in the range of 2.1-4.2 keV. Alternatively, if a factor of five decrease in collisionality is assumed, $\mathrm{T}_{\mathrm{e}} \leqslant 8 \mathrm{keV}$ is obtained.

In the above estimate for the electron temperature, it is implicit that the distribution of electrons in phase space is approximately constant in going from EBT-I to EBT-II. A similar assumption is embodied in the diffusive scaling criteria: 
$n \tau \sim A_{e r r}^{2} T_{e}^{3 / 2} ; A_{e f f} \cong A f_{A R E}$

The aspect ratio dependence comes from the fact that the neoclassical step size decreases as the major radius is increased. (In the limit of infinite aspect ratio, all the particle drift surfaces have circular cross sections, and there is no dispersion in the drift orbits.) Electrons tend to dominate the neoclassical energy loss because their collision frequency is larger than that of the ions by the square root of the ratio of the ion and electron masses. The relation $A_{\text {eff }} \cong A_{A R E}$ takes into account the fact that passing particles (of relatively high energy) contribute most to the neoclassical step size and that ARE coils can reduce this contribution.

Without ARE coils ( $f_{A R E}=1$ ) the aspect ratio is increased by a factor of 2.5 over EBT-I, and the electron temperature is increased by a factor of seven, so that $n \tau$ is increased from 1-2 $\times 1010$ $\mathrm{sec} / \mathrm{cm}^{3}$ in EBT-I to approximately $10^{12} \mathrm{sec} / \mathrm{cm}^{3}$ in EBT-II. With ARE coils, fARF lies in the range of $1.5-2.5$ and $n \tau$ is on the order of $10^{13} \mathrm{sec} / \mathrm{cm}^{3}$.

The power requirements fur these values of $n, \tau_{e}$, and $\tau$ can now be determined. The bulk heating power for EBT-II is $1.6 \mathrm{MW}$ while that of EBT-I is $60 \mathrm{~kW}$. The volume occupied by the toroidal core plasma is three to five times that of EBT-I, so that the average power density is increased by a factor of five to ten. The fraction of the total power absorbed (P) by the toroidal core plasma can be twice that of EBT-I, because the cold edge plasma can be less of an energy sink and because the ordinary mode is much more heavily damped at the higher temperatures of EBT-II.l Thus, the ratio of absorbed power densities is in the range

$P_{I I} / P_{I}=10-20$

The energy loss term which balances this input power density is

$\frac{3}{\overline{2}} \frac{n T_{e}}{l}=\frac{3}{\hat{2}} \frac{n^{2} \bar{T}_{e}}{n i}$.

With ARE coils the ratio of loss terms between EBT-II and EBT-I is less than or approximately equal to tên, so the power requirements are readily fulfilied. Without ARE coils the ratio of loss terms is somewhat less than 40. On this basis, the densities and temperalures without ARE coils would be slightly less than those given above (e.g., $\mathrm{n}=\sqrt{1 / 2} \cdot 5 \times 10^{13} \mathrm{~cm}^{-3}$ ).

In EBT-I, $T_{j} / T_{e} \cong 1 / 3$, which is just the value required to maximize the transfer of energy from electrons to ions via coulomb collisions. Thus, $T_{i}$ can be conservatively estimated from $T_{\dot{e}}$ However, there is a tendency for $T_{j}$ to become more nearly equal to $T_{p}$ becausse the longer lifetimes in FRT-IT permit increased equilibration.

Consideration is now given to some of the qualitative aspects of neutral beam injection in EBT-II. Because the ions can be heated directly, the ion temperature can exceed the electron temperature. This is thermodynamically impossible with ECH alone. Somewhat higher densities are possible because of reduced microwave cutoff limitations. (In the extreme case the microwaves need only sustain the hot electron rings and need not penetrate to the central core plasma.) Perhaps the most interesting feature is the possibility of controlling the electric field (E), because the neoclassical lifetime scales as $|E|^{2}$ for sufficiently large ambipolar electric field. 
One possibility for controlling the electric field stems from the fact that a second "state" having an electric field with the opposite polarity from that presently observed may be possible with neutral beam injection. To understand these two states it is necessary to realize that in the absence of an electric field the electron diffusive particle loss rate greatly exceeds the ion diffusive loss rate. This situation cannot persist in steady state because of conservation of charge. In EBT-I, the radial electric field is negative for radii less than that of the hot electron rings. The second state is characterized by a universally positive electric field.

For those used to determining the sign of the electric field by electrostatic arguments, the fact that the electric field in EBT-I is negative often comes as a surprise. The basic explanation for the negative electric field 1 ies in the particle drift motion. For this sign of the electric field, the $E \times B$ drift locally cancels the poloidal gradient- $B$ and curvature drifts for a class of ions on the tail of the distribution. Ions in this class have drift surfaces which have crescent- or bananashaped cross sections. The width of these banana-shaped orbits is sufficiently large that they can provide the dominant contribution to the neoclassical step size. The lowest energy for which this class of ions occurs is of the order of $e \Delta \phi$, and the fraction of ions in this class is approximately proportiona 1 to exp $-|e \Delta \phi| / T_{i}$. The magnitude of the electric field $(\sim \Delta \phi)$ adjusts so that there are sufficient particles in this class of ions for the ion and electron particle losses to be equal.

The state which is characterized by a positive electric field can be understood from electrostatic arguments. A positive electric field provides a radially inward force on the electrons which impedes their flow. For purely diffusive losses, the equivalent fluid statement is that electron particle fluxes proportional to density and temperature gradients are nearly canceled by a flux of electrons which is linearly proportional to the electric field (mobility). This allows the electron particle loss rate to be reduced to that of the ions.

For purely diffusive losses, this state with positive electric field tends to be unstable. ${ }^{2}$ For this case, the electric field tends to be proportional to gradients of density and temperature. For large electric fields, the transport coefficients are proportional to the inverse square of the electric field. Thus, the transport coefficients are roughly proportional to the inverse square of density and temperature gradients. Consequently, the diffusive mechanisms which are supposed to flatten sharp gradients become weakened, and the gradients tend to grow without bound. With neutral beam injection some of the losses are inherently nondiffusive, and the possibility exists for breaking some of the links in the positive feedback chain just described. (Even if this positive electric field turns out to be less than one might hope, there is still a benefit: an increased tendency for highly charged impurity ions to he exciuded from the central core plasma.)

Inevitably, neutral beam injection will be less than 100\% efficient in heating the toroidal core plasma. For high particle energyy and $v_{\|} / v$ in excess of 0.7 there are drift surfaces which intersect the walis of the device. With neutral beam injection, some of the fast ions will scatter in pitch angle (or $v_{\|} / v$ ) faster than they will slow down in energy, and some of these ions will drift to the wall. For an individual particle this is a much faster loss process than the random walk in nosition associated with diffusion. Thus, if there are sufficient ions undergoing this direct loss process, it can dominate the diffusive ion losses. (There are direct ion losses in EBT-I, but they are small because the distribution function of ions is small for energies in excess of the critical energy for the onset of this process.) For sufficiently energetic ions this process depends neither upon gradients of density and temperature nor upon the electric field.

Very preliminary calculations suggest that rather large electric fields could be generated by this process, which would lead to considerable enhancement of the confinement time. However, the stability of this state depends upon the detailed functional form of the transport coefficients. An adequate assessment awaits full implementation of the newly derlved liarisport coefficient3. 


\subsubsection{Confinement at Higher Values of Beta}

In EBT-I the value of $\beta_{\text {core }}$ [defined as $8 \pi n\left(T_{e}+T_{j}\right)$ core $/ B^{2}$ ] of less than $0.3 \%$ was significantly less than $\beta_{\text {annulus. }}$ For reactor operation, values of $\beta_{\text {core }}$ of $20 \%$ or more are desirable from an economic standpoint, so it is important in EBT-II to determine the necessary conditions for nonturbulent operation at higher values of $B_{\text {core }}$ than those obtained in EBT-I. If the anticipated scaling holds for EBT-II, values of $\beta_{\text {core }}$ as high as $10 \%$ can be achieved. A test of the ultimate beta limits may have to be deferred to a larger, more expensive device where the plasma confinement is high enough for $B$ core to approach $\beta_{\text {annulus }}$. Since $10 \%$ does exceed the value of $B$ annulus required for formation of the magnetic well, it may prove possible to test the critical beta value in EBT-II by reducing $\beta_{\text {annulus }}$. However, it would be fortuitous if this were to give definitive results.

A natural part of the study of beta limitations will be the search for the borders of the nonlurbulent operating regime. Experience with EBT-I indicates the importance of having firm control of the qas feed rates and particle influxes. Alsn, in ERT =I the importance of maintaining the symmetiy of the magnetic system and closure of the magnetic field lines to an accuracy of about $10^{-4}$ was shown. Errors below this quite feasible level have no observed effect. Above this level, lack of field line closure is readily compensated by a simple winding around the torus. Failure to compensate properly leads to a toroidal current in the torus, which causes slightly enhanced particle transport because of changes in the particle orbits, and to the onset of MHD instabilities caused by the plasma current parallel to B. (See Ref. 3 and Appendix B.) Appropriate compensation by vertical and horizontal field coils was observed to remove these problems in EBT-I, and simil lar coils will therefore he inrnrpnrated in the EBT-II design.

The beneficial contribution of the ambipolar electric fields has already been discussed. For EBT-II, there are three ways to control the potential and gain the improved confinement that results. First, the location of the resonant microwave heating region affects the potential strongly, so that varying the value of $B$ allows position control of the resonant zone. Similarly, use of additional microwave frequencies allows control over the resonant zone structure. Second, the compensation coils for field line closure provide some control over which particle orbits intersect the cavity walls near the mirror coils; therefore, control of the boundary conditions will determine the potential. Third, at the projected operating densities of EBT-II, it would be possible to provide local deposition of particles and energy by neutral beam injection. Such auxiliary heating changes the particle loss rates and therefore affects the potential.

\section{REFERENCES}

1. D. B. Batchelor, Status of the Theoretical Study of Microwave Heating in EBT. ORNL/TM-6320, Oak Ridge, Tennessee (to be published).

2. E. F. Jaeger et al., "Neoclassical Transport in the ELMO Bumpy Torus," Phys. Rev. Lett. $40(13)$, $866.869(1.978)$.

3. B. H. Quon, R. A. DandT, P. L. Colestock, and H. Ikegami, Effects of Global Field Error on ELMO Bumpy Torus, ORNL/TM-6075, Oak Ridge, Tennessee (October 1977). 


\section{DESIGN DESCRIPTION}

\section{1 MAJOR ELEMENTS}

The EBT-II experiment will be located on the second floor of B1dg. 9201-2 at the Y-12 Plant, as shown in Fig. 3.1. Figure 3.2 shows the layout of the equipment making up the EBT-II device.

Plan and elevation views of the EBT-II device are presented in Figs. 3.3-3.5. Table 3.1 presents the pertinent physical characteristics of the device. The EBT-II device is a 10.4-m-diam torus vacuum vesse1, composed of 48 magnet dewars, each containing a superconducting mirror coil, alternating with 48 wedge-shaped cavities. Primary pumping of the vacuum vesse1 is accomplished through the use of 48 cryocondensing pumps, one on each cavity, around the periphery of the torus. A roughing system, composed of cryosorption and turbomolecular pumps, is utilized for initial pumpdown of the vacuum vessel, regeneration of the cryocondensing pumps on the vacuum vessel, and evacuation of the superconducting magnet dewars.

The steady-state plasma is contained by the magnetic field generated by the mirror coils. Each coil consists of a liquid helium cooled superconducting winding enclosed in a stainless steel case. A system of 48 aspect ratio enhancement (ARE) coils is also provided for the purpose of adjusting the effective aspect ratio of the device. The ARE coils encircle each vacuum cavity and are of water cooled copper design.

Stabilization and partial heating of the toroidal plasma is provided by the injection of microwave energy at two or more frequencies into the toroidal vessel. The microwave power is transmitted from the power source (gyrotron) to the toroidal cavity via oversized waveguides having symmetrical connections to each cavity region. The gyrotron is a microwave power oscillator based upon principles of the cyclotron resonance masers.

Additional plasma heating will be provided by the addition of four neutral beam injectors, symmetrically located around the major radius of the device and vertically suspended from the roof of the enclosure. The neutral particles are perpendicularly injected into the cavity sections. An energy dump is provided on the cavily, diametrically opposite the injector, to absorb the beam not trapped by the plasma.

The torus structure is mounted on a concrete ring supported by concrete columns. The torus midplane is about $3.7 \mathrm{~m}$ above the operating floor. This arrangement permits access to the area inside the torus, which will be used for ancillary equipment. A mezzanine floor $1.25 \mathrm{~m}$ below the torus midplane provides operating access to diagnostic equipment.

The entire torus structure $\mathrm{w}_{1} \mathrm{Il}$ be surrounded by a $60-\mathrm{cm}$-thick concrete biological radiation shield. Entrance will be through a "darkroom maze" designed to attenuate the refiected radiation. A 10-cm-thick lead roof covers the structure. A control room adjacent to the shielded structure will house all machine control components and diagnostics.

Mechanical equipment associated with machine operation will be located reasonably close to the device where space is available. The helium refrigeration equipment required for cryogenic service will be located on the rouf of the building housing the experiment. Helium gas storage tanks wil1 be located outside the building to accommodate the gas equivalent content of the liquid helium system.

The gyrotron power supplies will be sheltered in an addition to the existing building. The vacuum pumping equipment, the gyrotrons for microwave power generation, and the microwave power transmission network will be located inside the experiment enclosure. Magnet power supplies and energy dumps will be located on the shielded roof. 
ORNL/DWG/FED 78-760

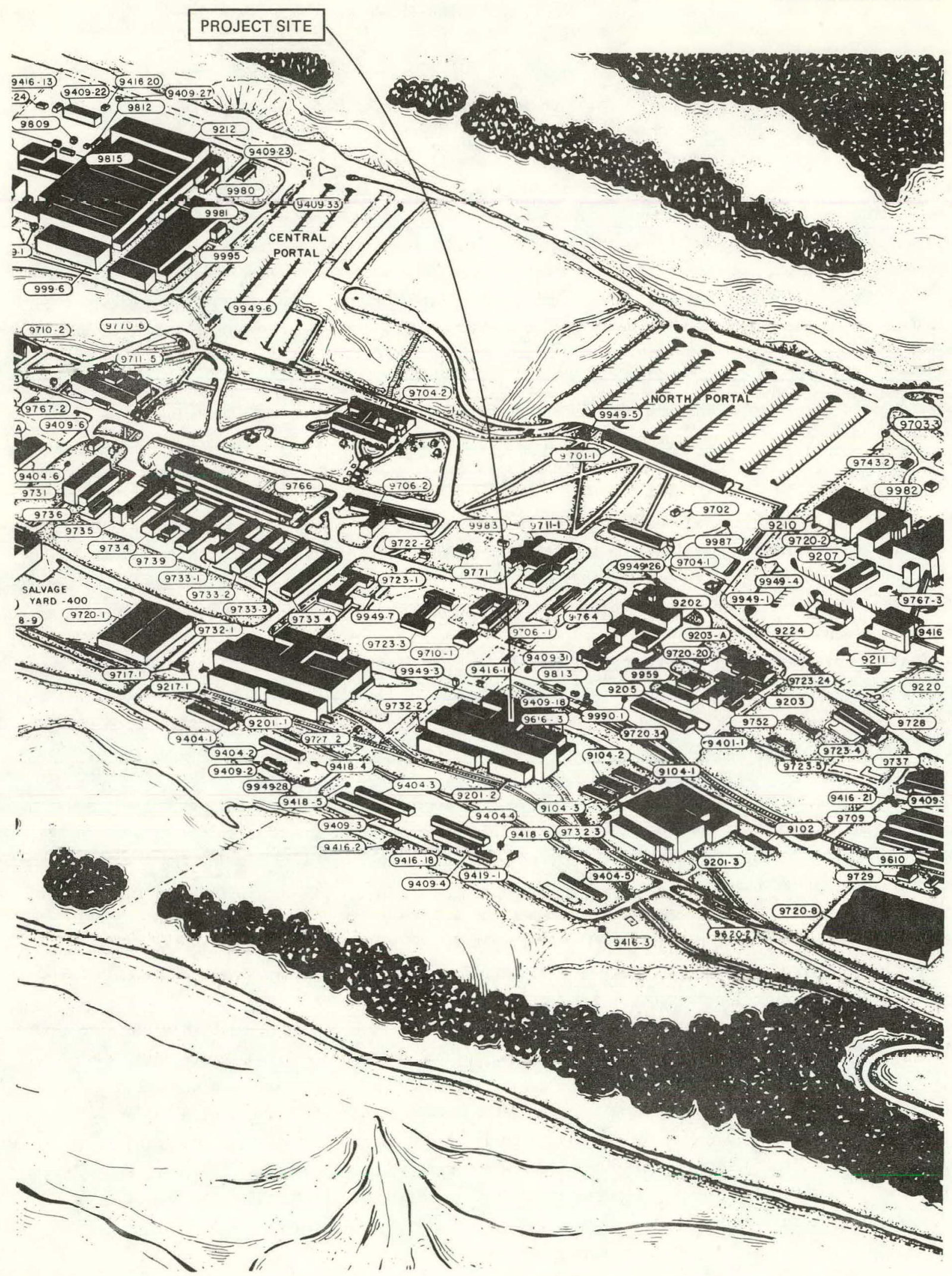

Fig. 3.1. EBT-II project location. 


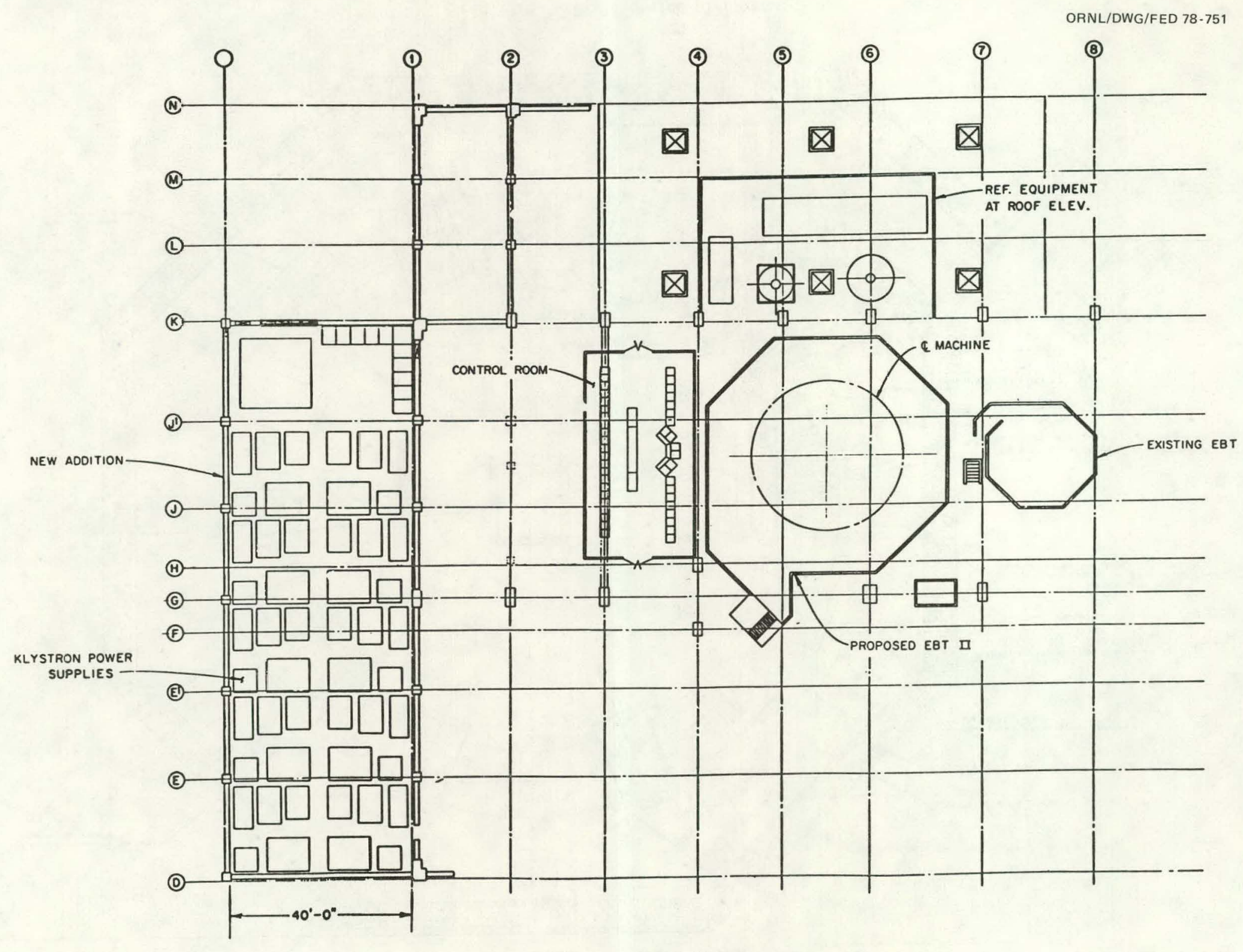

Fig. 3.2. EBT-II equipment layout. 


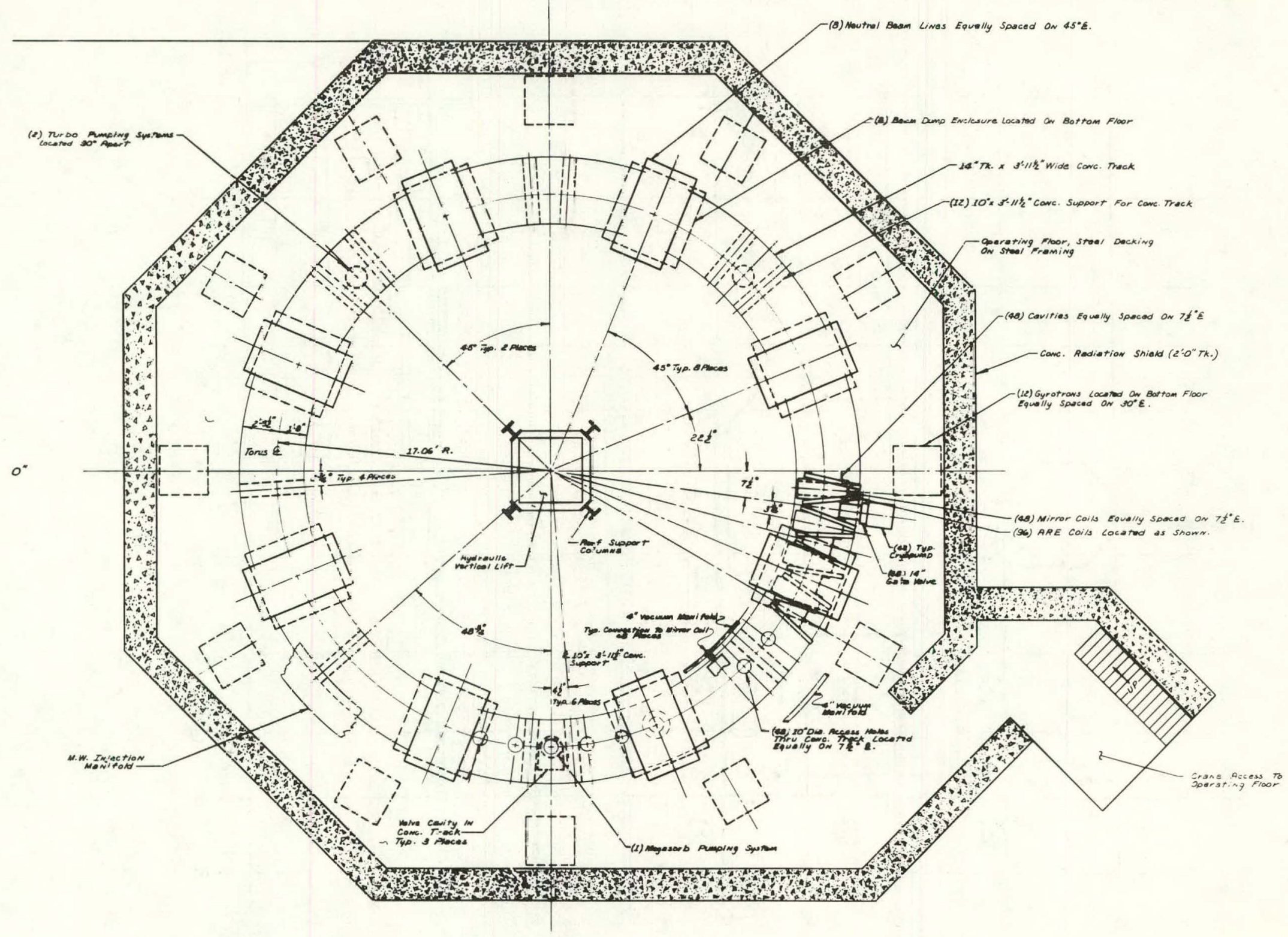

Fig. 3.3. EBT-II device plan view. 


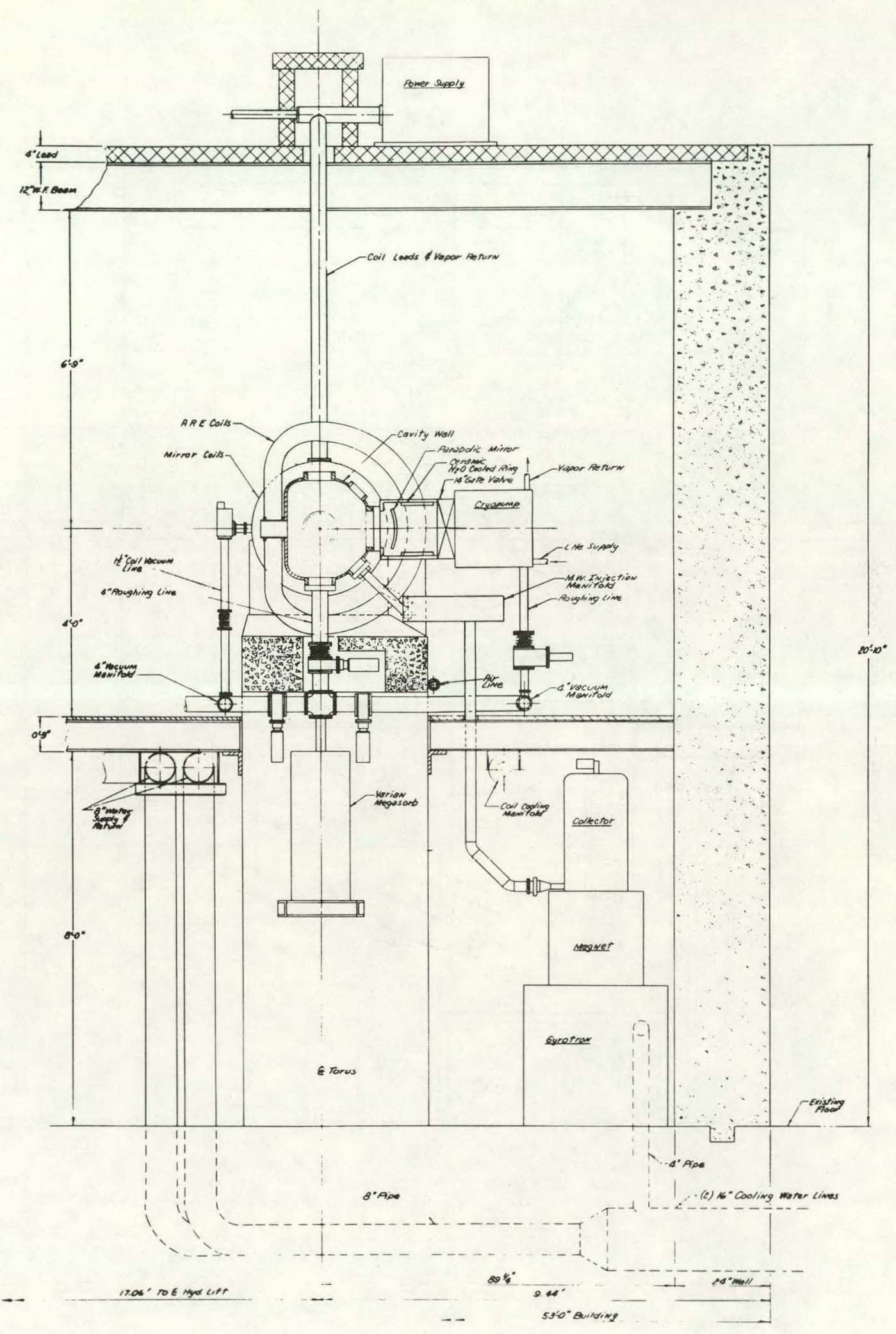

Fig. 3.4. Section elevation through typical cavity. 
ORNL/DWG/FED $78 \cdot 798$

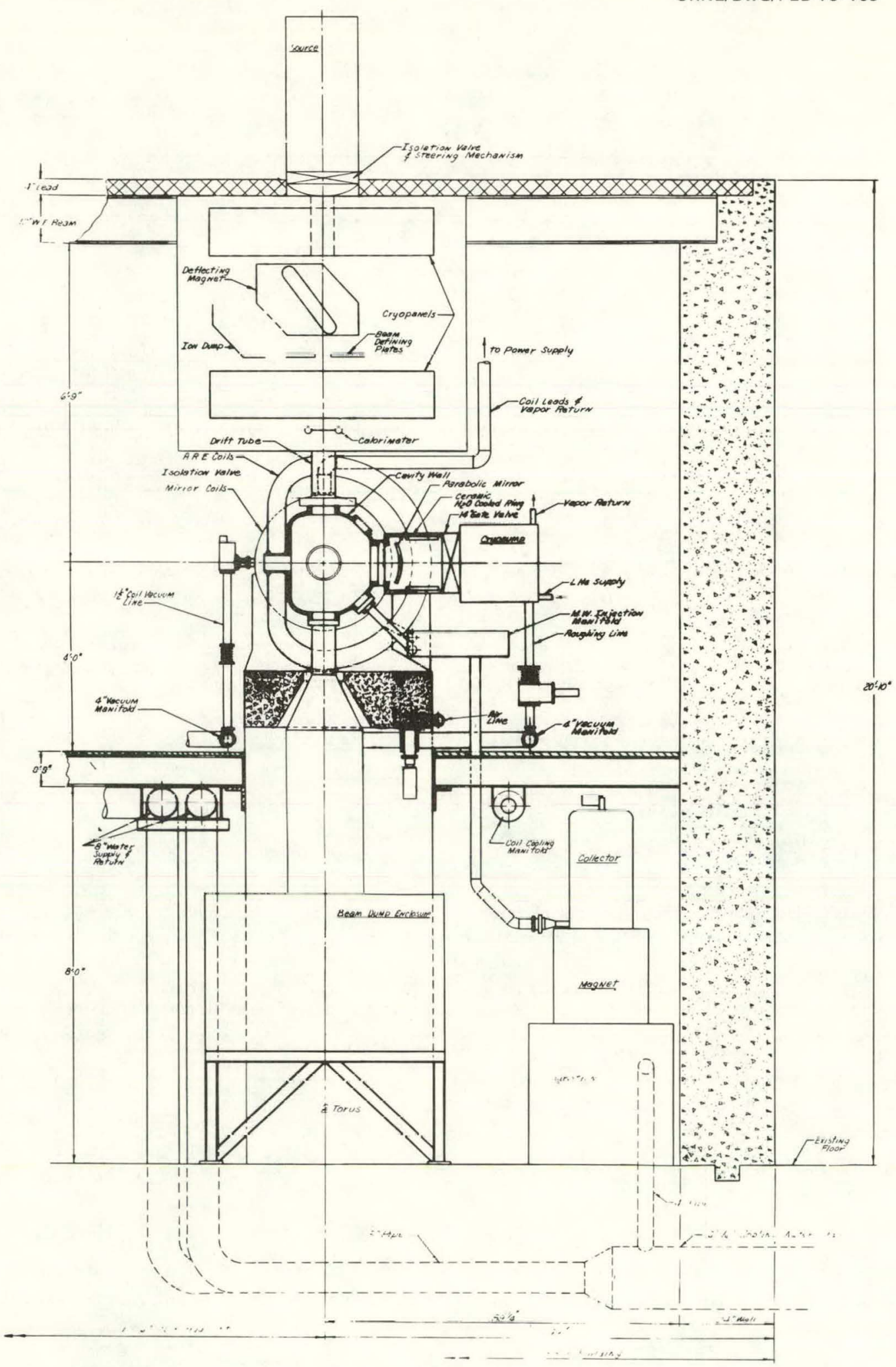

Fig. 3.5. Section elevation through neutral beam input cavity. 
Table 3.1. Physical characteristics of EBT-II

Torus

Torus major radius

Number of sections

Cavities

Dewars

Cavity volume

Dewar throat volume

Vacuum port diameter

Neutral beam injector port diameter

Mirror coils

Number of coils

Mean diameter

Winding area

Current density

Ampere-turns

Maximum field at winding

ARE coils

Number of coils

Mean perimeter

Winding area

Current density

Ampere-turns

Magnetics

Mirror ratio

Coil central field

Cavity central field

Torus vacuum system

Primary pumping system

Roughing system

UT timate pressure (no plasma)

Pumping speed per port

Net pumping speed

Neutral beam system

Number of injectors

Type of injection

Beam energy

Current

Source power

Microwave system

\section{$5.2 \mathrm{~m}$}

48

48

$4.5 \mathrm{~m}^{3}$

$0.97 \mathrm{~m}^{3}$

$24 \mathrm{~cm}$

$15 \mathrm{~cm}$

\section{8}

$55.2 \mathrm{~cm}$

$352 \mathrm{~cm}^{2}$

$6650 \mathrm{~A} / \mathrm{cm}^{2}$

$2.366 \times 10^{6}$

$7.8 \mathrm{~T}$

\section{6}

$3.24 \mathrm{~m}$

$102.2 \mathrm{~cm}^{2}$

$1505 \mathrm{~A} / \mathrm{cm}^{2}$

153,800

\section{ARE coils off \\ 1.95 \\ $5.85 \mathrm{~T}$ \\ $3.0 \mathrm{~T}$}

48 25,000-1iter/sec (hydrogen) cryocondensing pumps

1 84-1iter/sec cryosorption pump

2 1500-Iiter/sec turbomolecular pumps

1 50-cfm mechanical pump

$2 \times 10^{-7}$ torr

4050 liter/sec (hydrogen)

195,000 liter/sec (hydrogen)

4

Perpendicular

$20 \mathrm{keV}$

$12.5 \mathrm{~A}$

$1 \mathrm{HW}$

\begin{tabular}{cc} 
Resonant heating & Profile heating \\
\cline { 2 - 2 } & 4 \\
$120 \mathrm{GHz}$ & $90 \mathrm{GHz}$ \\
$1.6 \mathrm{MW}$ & $800 \mathrm{~kW}$
\end{tabular}

Number of gyrotrons

Microwave frequency

Total input power 


\section{1 .1 Vacuum Torus}

The aluminum vacuum dewars for the superconducting mirror coils are installed in a circular geometry with equal spaces between each coil and are attached to the torus support ring. The centerline of the coils is located on a radius of approximately $5.2 \mathrm{~m}$. Details of the design of the dewar will be presented later. Aluminum wedge-shaped spool pieces are inserted from the outer perimeter of the torus into the spaces between the vacuum dewars. Each side of the spool piece has a mounting flange (with 0-ring) through which machine screws are engaged into tapped holes in the dewar wall. The 0-rings are compressed by equal torquing of these bolts to effect a vacuum-tight seal between coil cases and spool pieces.

Each spool piece is noncircular in cross section, having a straight vertical side on the inner radius of the machine and a circular shape around the remainder of the torus. Two flanged ports are located on the vertical centerline, one directly above the other; a third and larger port is on the equatorial centerline and will be used for vacuum pump attachment. On either side of this vacuumm port, at a $45^{\circ}$ angle to the horizontal, are ports for microwave injection and for attachment of diagnostics. There is an additional rectangular slit penetration on the inner radius of the torus at the midplane for diagnostics.

The assembly of the two types of modules into a vacuum-tight torus requires caution in order to overcome the accumulation of fabrication tolerances which would prevent achieving a vacuum-tight chamber. The exact major radius of the torus will be left a dependent variable. Freedom of radial movement of the vacuum dewars must be permitted until the torus has been completely assembled; then the dewars will be fastened to the support structure. The vacuum dewars and spool pieces are water cooled to prevent significant temperature changes during machine operation.

\section{1 .2 Magnetics}

The EBT-II magnetic system, shown in Figs. 3.6 and 3.7 , consists of a system of toroidal magnets (mirror coils) and a system of aspect ratio enhancement (ARE) coils. The mirror coils generate a toroidal magnetic field to contain the stegadv-state plasma, and the ARE coils provide a method for adjusting the effective aspect ratio of the device without altering the mechanical aspect ratio. Aspect ratio enhancement is desirable, as noted in Sect. 2, because particle confinement time is proportional to the square of the aspect ratio.

Aspect ratio enhancement is accomplished by encircling the plasma with current loops that are positioned to induce a field curvature opposite that produced by the mirror coils (toroidal field). The aim of these coils is to cause a significant stretching of flux lines near the inner bore of the device to make them nearer the length of the outer flux lines. This is a characteristic of larger aspect ratio devices. It is desirable, therefore, to have the current element as close to the plasma as possible at the inner bore and far enough away elsewhere to have a minimal effect on the plasma. This is the basis for the D-Ehaped $\Lambda R E$ coil.

The system shown in Figs. 3.6 and 3.7 produces an aspect ratio enhancement factor of approximately two when the ARE coils are operating at a current level of $6.5 \%$ of the mirror coil current and in an opposing direction to the mirror coil current. Figure 3.8 shows the effect of the ARE coils on the toroidal field flux lines, and Fig. 3.9 shows their effect on the profile and primary resonance mod-B contours. As can be seen, significant stretching is attainable. For the conditions mentioned above, on-axis fields of $5.40 \mathrm{~T}$ in the coil throat and $2.45 \mathrm{~T}$ in the mirror cavity are produced, resulting in a mirror ratio of 2.20. With the ARE coils deenergized, these on-axis fields are $5.85 \mathrm{~T}$ and $3.0 \mathrm{~T}$, respectively, yielding a mirror ratio of 1.95 . 


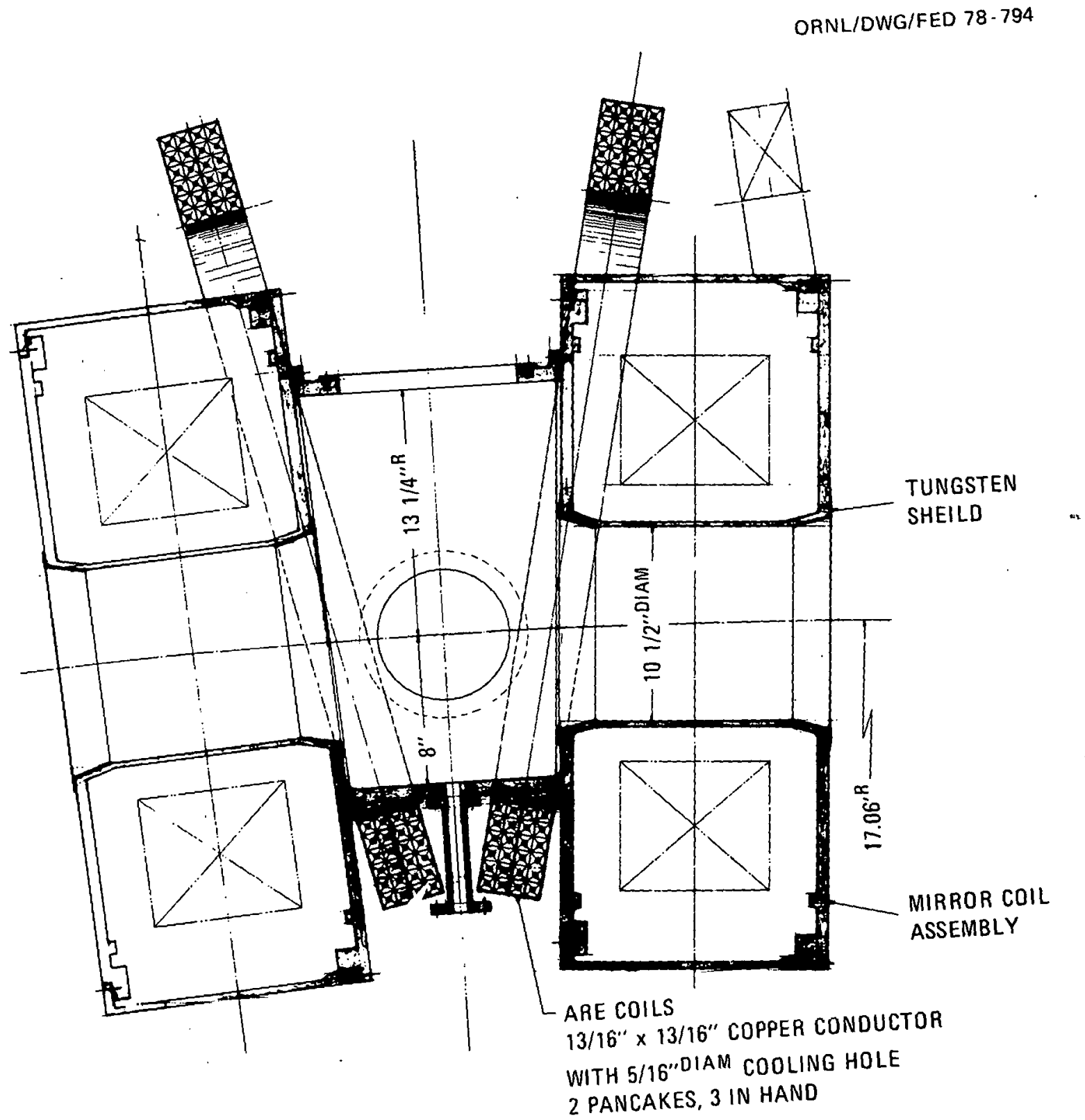

Fig. 3.6. Section plan through torus. 


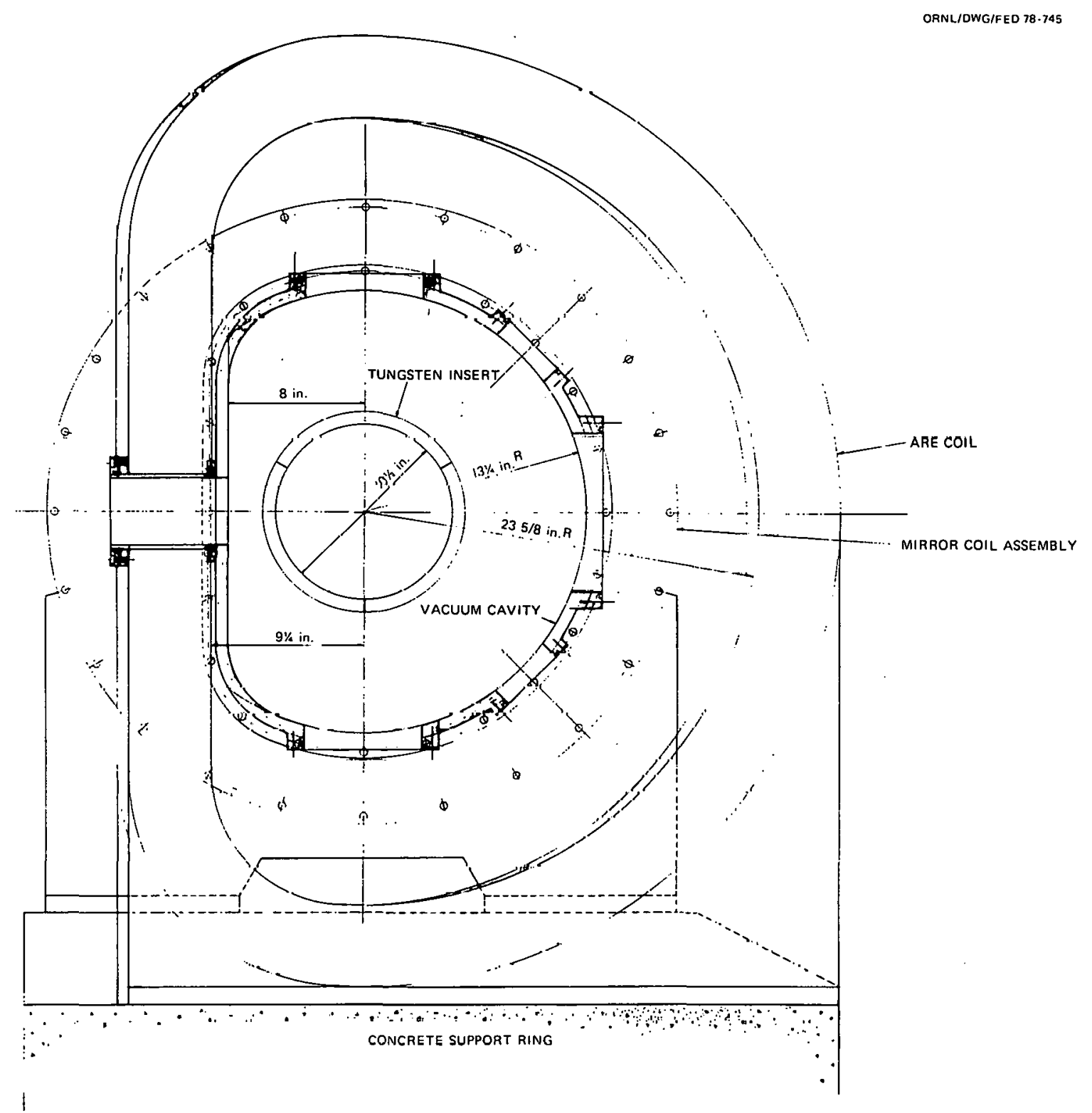

Fig. 3.7. Section elevation through torus. 


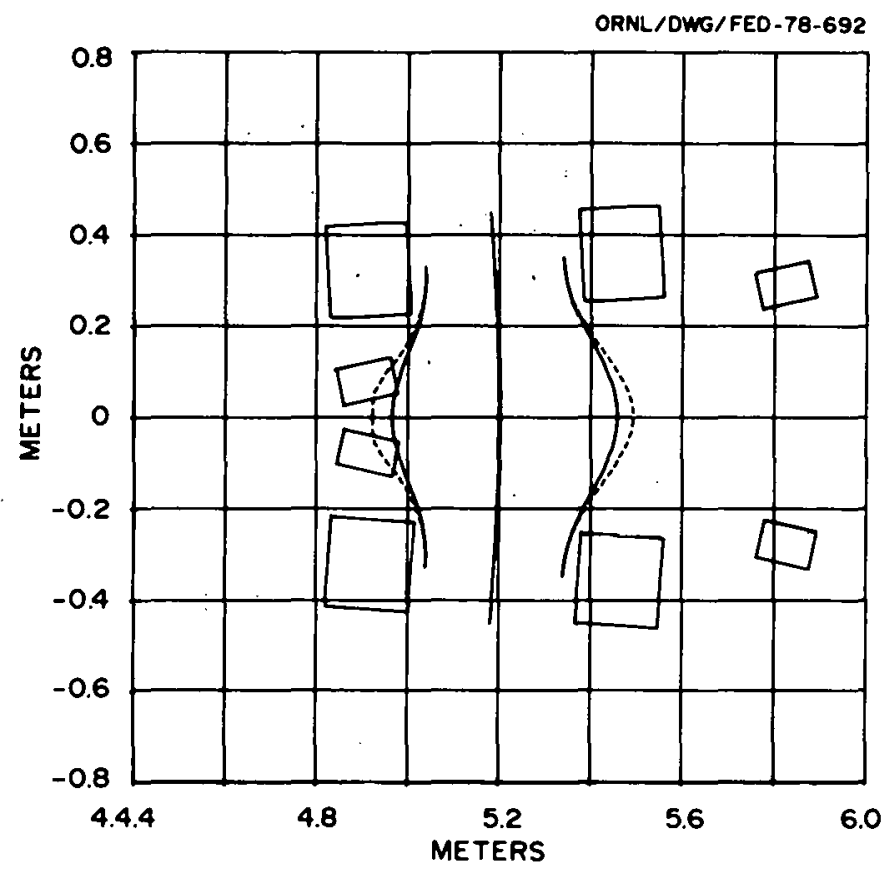

Fig. 3.8. ARE coil effect on magnetic flux lines. Solid lines: ARE coils deenergized; dotted lines: ARE coils energized.

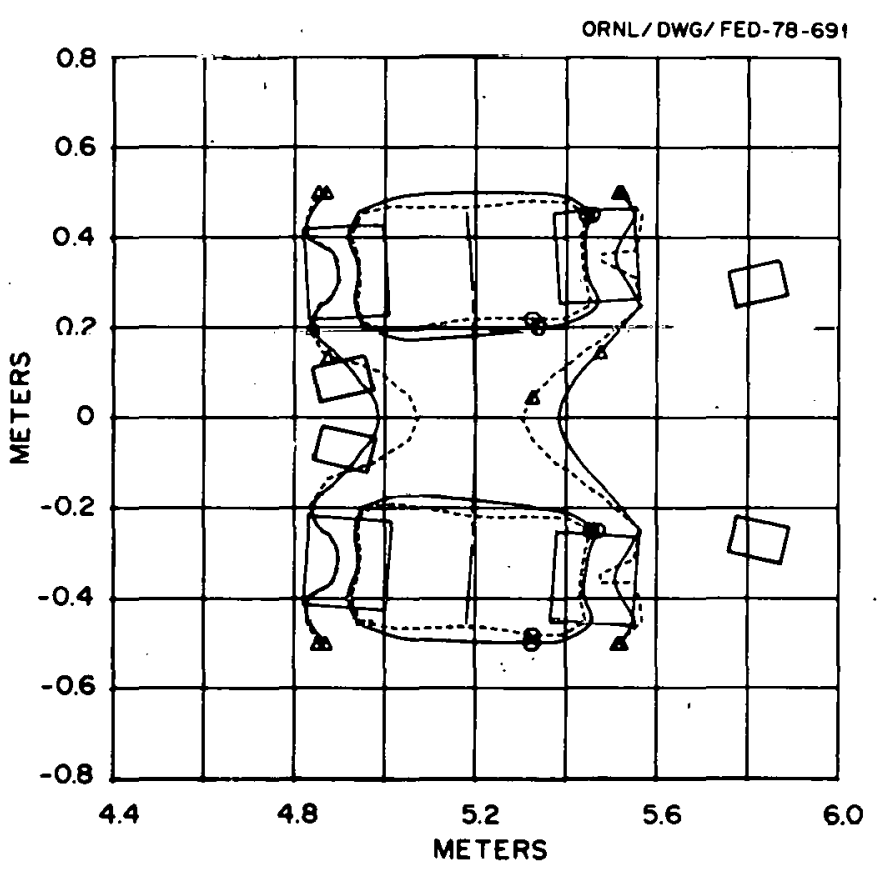

Fig. 3.9. ARE coil effect on mod-B lines. Solid lines: ARE coils deenerqized; dotted lines: ARE rnils energized. 


\subsubsection{Mirror coil system}

Configuration. The superconducting mirror coil system, shown in detail in Figs. 3.10 and 3.11 and described in Table 3.2, is composed of the following elements: (1) superconducting windings, (2) stainless steel coil case, (3) liquid nitrogen cooled shield, (4) super insulation layers, (5) water cooled uranium gamma shield, (6) internal stainless steel support structure, and (7) water cooled aluminum vacuum dewar housing.

The exact conductor configuration for the superconducting mirror coil winding will be determined from a test program, as described in Sect. 3.1.2.4. It is assumed that the winding is a potted NbTi coil with helium cooling passages around three sides of the rectangular coil section. The coil winding is contained in a stainless steel can with strips of compressive insulation transferring the coil magnctic loads to the coil case.

The mirror coil is completely surrounded by a liquid nitrogen barrier with sheets of reflective insulation on both sides to minimize thermal radiation leaks to lhe mlror cuil, which is at liquid helium temperature. A water cooled uranium gamma shield is alsu provided on the inner bore and diung the two sides of the coil to protect the coil from plasma emitted photon radiation. This radiatiun shield is discussed in more detail below.

The coil case has an integral stainless steel ring on each side of the case near its outer diameler. which is tightly wound with stainless steel ribbon approximately $1 / 2$ in. wide and 1 mil thick. This winding is built up to a height of about $1 / 2$ in. It is through these laminated rings that the gravity load and centering load are transferred to the dewar housing. The purpose of these wound laminations, which have a thermal conductivity approximately one fiftieth that of solid stainless steel, is to reduce thermal conductivity from the $4.2 \mathrm{~K}$ coil case to the liquid nitrogen cooled support structure.

The coil case is connected to the support structure through threaded studs which are tightened to center the coil case accurately in the dewar housing. The support structure has a machined rectangular slot which fits over a ring integral with the dewar housing. When the studs are tightened against the bands around the coil case, the inner radius of the slot bears on the bottom edge of the ring su lhat the coil is securely held in the dewar. Using 25 inreaded studs un eacli side of the coil can, the loads imposed by both gravitational load and centering force put a compressive load on these studs that is insignificant.

A rib approximately $1 \times 1 \mathrm{in}$. In cross section is located around the outer perimeter of the coil case. The split support structure bears against this rib through laminated washers, which present a high resistance to heat loss from the $77 \mathrm{~K}$ structure to the $4.2 \mathrm{~K}$ coil case. The two sides uf llie dewar housing are clamped against this bucking ring through the washers. While the clamps are in place, the outer housing of the coil case is slipped into place and bolted. The final coil case closure weld is made on the inside bore of the coil.

Power is supplied to the mirror coils via helium vapor cooled leads from individual power supplies located on the roof of the torus enclosure. Details of the electrical lead penetration through the vacuum dewar are shown in Fig. 3.11.

Gamma radiation shield. The superconducting mirror coils of the EBT-II device are to be shielded from high energy bremsstrahlung radiation by a nickel plated depleted uranium shield located on the sides and inner bore of each coil as shown in Fig. 3.10. The coil side shield is composed of 1.5-in.thick material, and the inner bore shield is made from 1 -in.-thick material. A removable lunisten (oir other metal) liner is also provided at the corners of the vacuum dewar throat to prevent erosion of the aluminum due to electron bombardment. Each shield contains cooling tubes through which demineralized water is circulated to extract a portion of the microwave energy added to the plasma. Peak temperature rises are to be 1 imited to approximately $100^{\circ} \mathrm{C}$. 


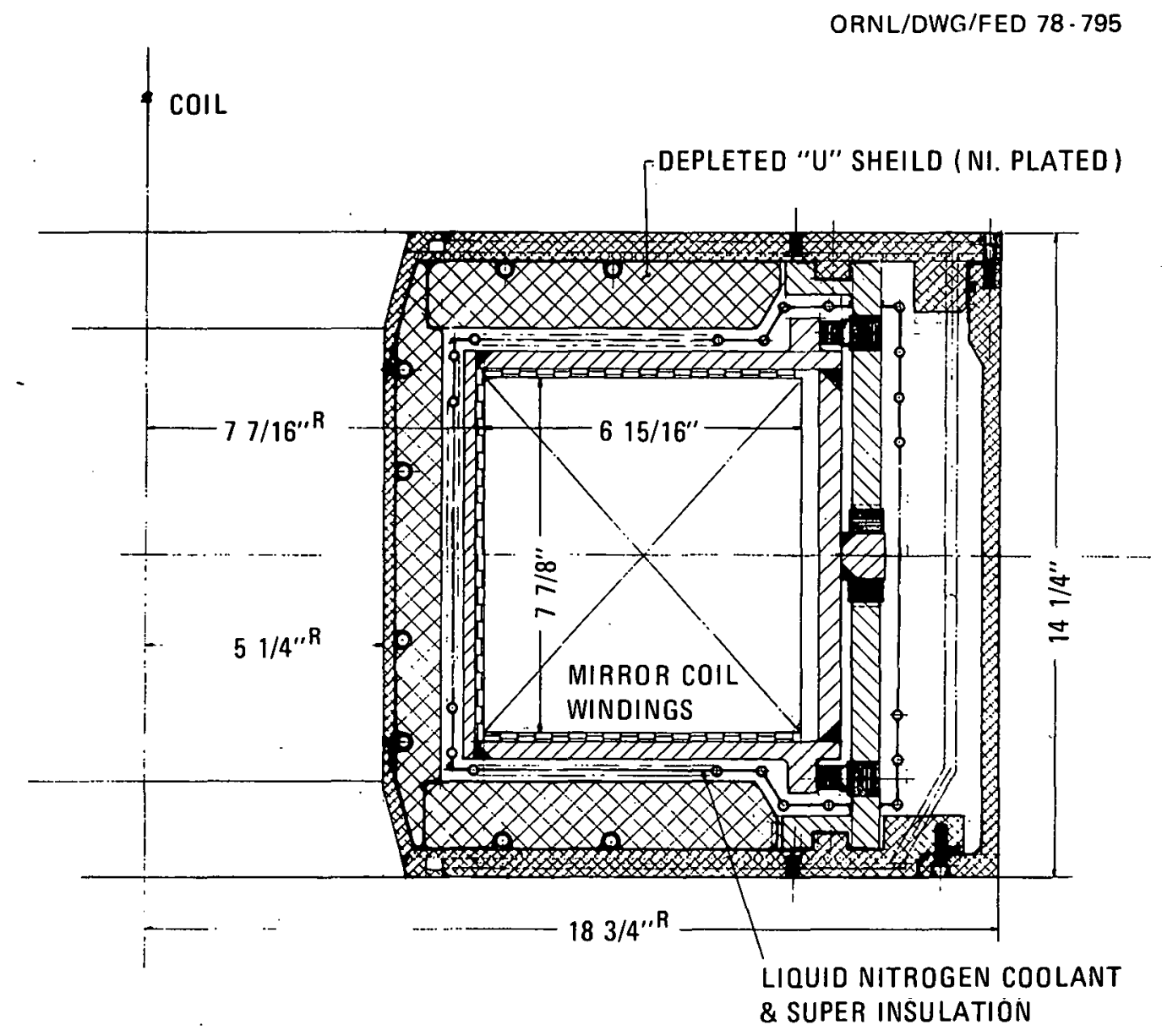

Fig. 3.10. Section plan through mirror coil. 


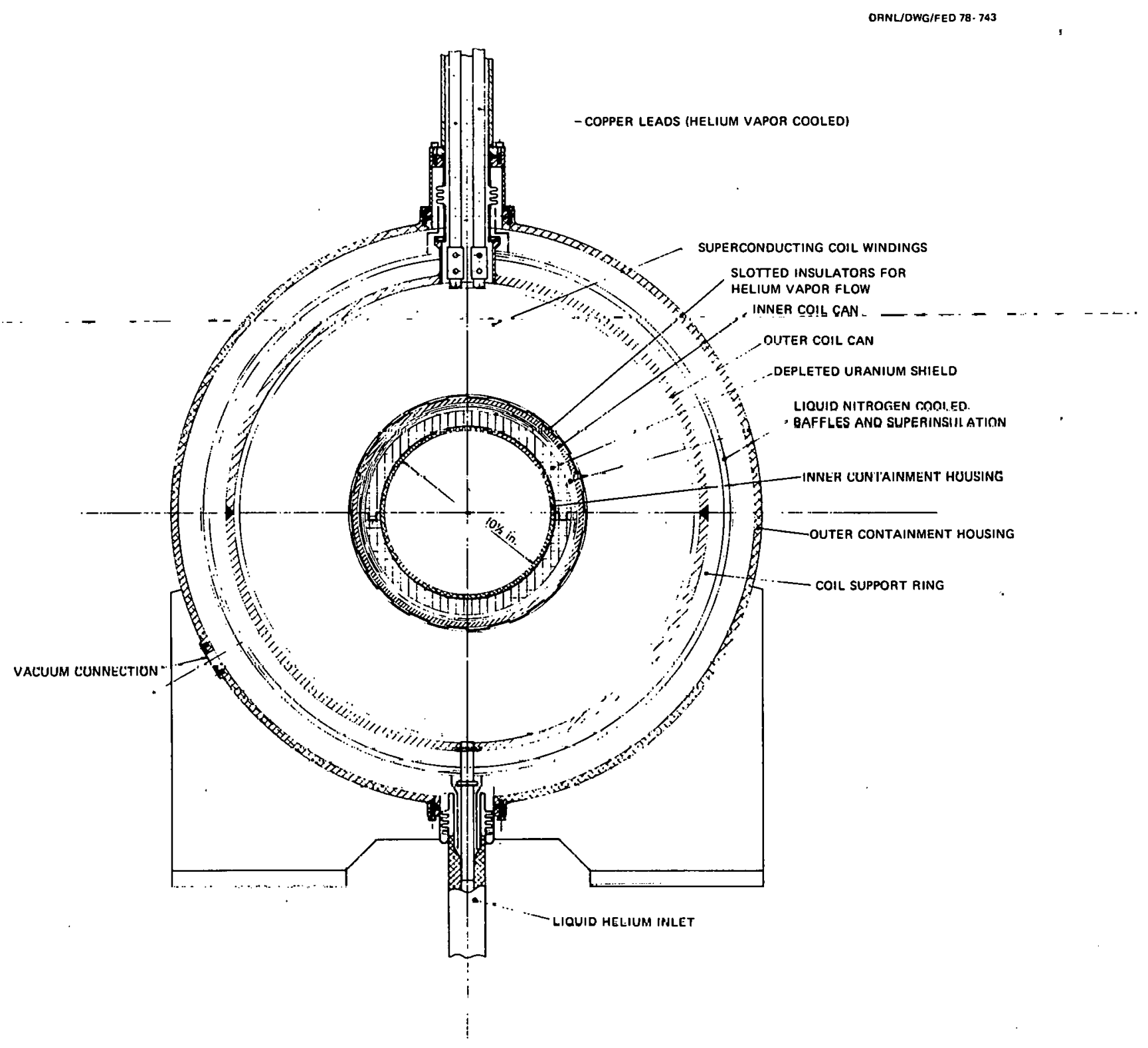

Fig. 3.11. Section elevation through mirror coil. 
Table 3.2. Mirror coil parameters

\begin{tabular}{ll}
\hline Number of coils & 48 \\
Coil shape & Circular \\
Peak field & $7.8 \mathrm{~T}$ \\
Operating temperature & $3.5 \mathrm{~K}$ \\
Winding inner diameter & $18.8 \mathrm{~cm}$ \\
Winding outer diameter & $36.4 \mathrm{~cm}$ \\
Winding width & $20 \mathrm{~cm}$ \\
Average current density (winding) & $6650 \mathrm{~A} / \mathrm{cm}^{2}$ \\
Ampere-turns & $2.37 \times 10^{6}$ \\
Cool ing mode & Pool-boiling \\
Stored energy (per coil) & $1.5 \mathrm{MJ}$ \\
Inductance (per coil) & $0.98 \mathrm{H}$ \\
Case material & $5 t a i n l e s s ~ s t e e l$ \\
Winding weight (per coil) & $540 \mathrm{~kg}$ \\
Case weight (per coil) & $166 \mathrm{~kg}$ \\
\hline
\end{tabular}

Uranium is used because its high specific gravity utilizes space most efficiently for attenuating the bremsitrahlung radiation emitted due to the interaction between electrons in the megaelectron-volt range and the aluminum. While other materials (such as gold, platinum, iridium, or tungsten) have comparable densities, they are impractical from an expense or refractory point of view; the uranium metal is worked routinely in the Oak Ridge complex and is available for handing costs only. The presence of uranium, however, requires that certain precautions be exercised with the design and operation of EBT-II because of neutron capture in $238 \mathrm{U}$ with its resulting radioactivity and because of the potential for a pyrophoric reaction of uranium upon exposure to an oxidizing agent. These potential problem areas will be eliminated or minimized in the EBT-II by control of the experiments and of the environment that might interact with uranium shield.

The plasma experiments will be performed with hydrogen and will not yield fusion neutrons. If other experiments are performed with deuterium, a detailed radiation safety analysis will be performed and reviewed to ensure adequate neutron shielding and to account for the D-D neutron induced activity in the depleted uranium shield.

The depleted uranium shield is enclosed in the aluminum dewar casing, which will contain cooling tubes through which demineralized water is circulated to remove the energy absorbed from radiation and particle bombardment. The aluminum casing will prevent direct contact between the uranium and the surrounding gas. Normally, the chambers around the uranium shielding will contain either plasma or vacuum insulation of the magnet cryogenic system. Hence, the only time any oxygen could reach the uranium metal is during a maintenance period when the system is cold. Since the uranium shielding is solid metal, there would be no pyrophoric reactions in the cold conductor.

The $x$-ray heating of the superconducting mirror coil could possibly cause coil quenching. Hence, an estimate of the heat input to the coil from plasma radiation was made. In estimating this heat load, the following assumptions were made.

1. The hottest conceivable electron annulus is $2 \mathrm{MeV}$.

2. The maximum microwave power dissipated in plasma rings is $P_{\mu}=1$ MW (one-half of total power).

3. All of the electron energy is converted to bremsstrahlung in the low $Z$ aluminum 1 iner.

4. All photons not attenuated by the uranium shield are dissipated in the coil. 
5. All annulus electrons are lost on dewar throat edges (see Fig. 3.12), thus making the edges intense ring sources of bremsstrahiung.

6. $F_{A}=0.2$ and $F_{B}=0.5$ are the fractional solid angles subtended by the coil windings. An additional factor of one-half was incorporated into $F_{B}$ to account for geometry.

7. There will be $3.8-\mathrm{cm}$-thick side shielding and $2.5-\mathrm{cm}$-thick inner bore shieiding.

8. The conversion efficiency of electron energy into photon energy is $C_{\text {ex }}=2.9 \%$ (nonrelativistic).

9. The uranium attenuation factors are $A_{B}=69$ for $2.5-\mathrm{cm}$-thick shielding and $A_{A}=350$ for $3.8-\mathrm{cm}-$ thick shielding.

Based on the above assumptions, the heat input to the coils can be calculated as follows:

$$
\begin{aligned}
P_{\alpha} & =P_{\mu} C_{e x}\left(\frac{F_{A}}{A_{A}}+\frac{F_{B}}{A_{B}}\right) \\
& =\left(1 \times 10^{6}\right)(0.029)\left(\frac{0.2}{350}+\frac{0.05}{69}\right) \\
& =37.5 \mathrm{~W}(48 \text { coils }) \\
& =0.78 \mathrm{~W} / \text { coil. }
\end{aligned}
$$

Although sufficient conservatism was incorporated into the shield design, provisions have been made for a backup system in the form of a shielded, U-shaped, water cooled skimmer which traverses a portion of the cavity periphery and can be assembled through an existing access port.

\begin{tabular}{|c|c|}
\hline Electrical lead loss. & ZuZ litêrs/hr \\
\hline Magnet joint resistive loss & $4 W$ \\
\hline \multicolumn{2}{|l|}{ Thermal radiation loss } \\
\hline Liquid helium surface 3 & $5 \mathrm{~W}$ \\
\hline Valves & $400 \mathrm{~W}$ \\
\hline Plasma gamma radiation & $50 \mathrm{~W}$ \\
\hline \multicolumn{2}{|l|}{ Conduction losses } \\
\hline Structural & $400 \mathrm{~W}$ \\
\hline Instrument leads & $30 \mathrm{~W}$ \\
\hline Total & $889 \mathrm{~W}+2021 \mathrm{jtchs} / \mathrm{hr}$ \\
\hline Totol plus $50 \%$ contingency & $1300 \mathrm{~W}+3001 \mathrm{itcrs} / \mathrm{hr}$ \\
\hline
\end{tabular}

Refrigeration requirements. The refrigeration requirements for the 48 superconducting mirror coils have been estimated at $1300 \mathrm{~W}$ at $4.2 \mathrm{~K}$ and $300 \mathrm{liters} / \mathrm{hr}$ of liquid helium for lead cooling. The heat losses are attributed to the following sources.

Coil quench protection. Each coil has an effective inductance of $0.98 \mathrm{H}$ and a stored energy of about $1.5 \mathrm{MJ}$ (72 MJ for 48 coils). The characteristic discharge time of a coil is about $2 \mathrm{sec}$ or longer. Accordingly, the coils will have an independent power supply protected by a diode in parallel and a $0.33-\Omega$ resistor in series. An automatic quench detector (voltage tap) for dump control will actuate the current dump system. Maximum coil temperatures less than $77 \mathrm{~K}$ will be reached during the quench and maximum terminal voltages will be less than $500 \mathrm{~V}$. These conditions impose no problems for superconducting magnets. 


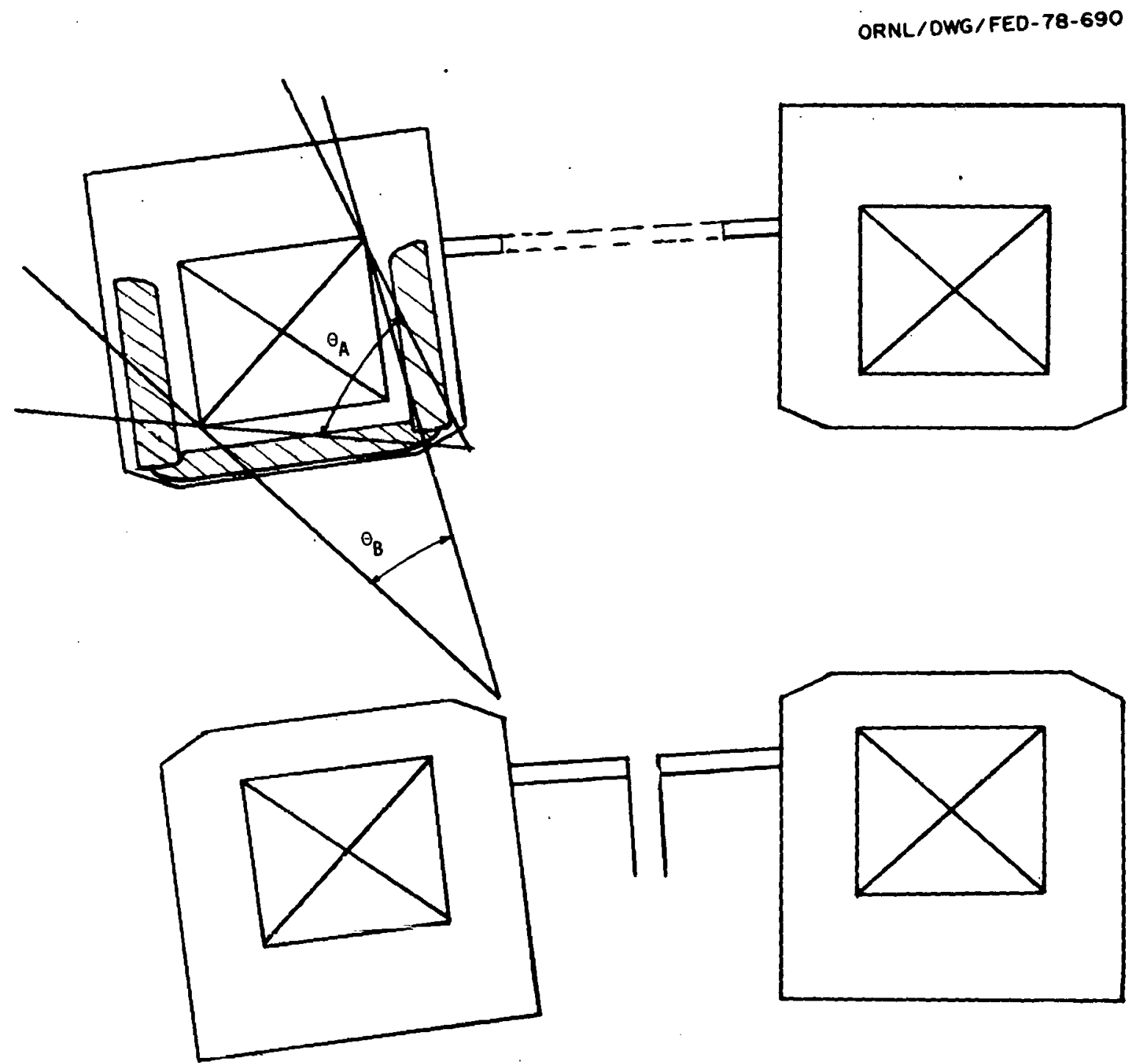

Fig. 3.12. Definition of shielding calculation nomenclature. $F_{A}=\theta_{A} / 360 ; F_{B}=\theta_{B} / 360$. 


\subsubsection{ARE coil system}

Configuration. The EBT-II ARE coil design is shown in Figs. 3.6 and 3.7. The design presented in this report assumes normal water cooled copper coils. It appears probable that superconducting ARE coils can be used in the same position as the copper coils. Such superconducting coils will be studied in detail later. The ARE coil parameters are listed in Table 3.3.

Each ARE coil consists of 24 turns of 13/16 $\times 13 / 16-i n$. copper with an integral 5/16-in.-diameter hole for cooling water circulation. The coils are wound three-in-hand into two double pancakes, which gives a coil cross section four conductors wide and six conductors thick. The 24 turns of the ARE coil are connected in series electrically. This winding scheme yields six coolant paths connected in par-

alle1. Turn insulation is provided by epoxy-fiberglass material with the coil being bonded into a unit by the epoxy compound. Each ARE coil is enclosed in a 0.25-in.-thick bolted stainless steel case. An ARE coil current equivalent to $6.5 \%$ of the mirror coil ampere-turns (see Table 3.2) requires magnet power for the $96 \mathrm{ARE}$ coils of about $15 \mathrm{MW}$. Water flow is adequate to cool the coils for up to $20 \mathrm{MW}$ of uuwer dissipation.

The ARE coils are D-shaped in order to position the winding as close as possible to the plasma on the torus inner radius. The vertical bore dimension of the coil is as small as possible in order to minimize the neutral beam drift tube length. The horizontal bore diameter is relatively large to provide necessary assembly clearance for insertion of the spool piece. This process involves moving the ARE coils as far as possible toward the machine center and making the connections between the spool pieces and the vacuum dewar walls. When the vacuum torus assembly is complete, the ARE coils are clamped into their support structure, moved into operating position, and secured.

Power is supplied to the ARE coils via water cooled buswork from existing motor-generator sets. Electrical and hydraulic connections for the ARE coils are made outside the coil case.

Table 3.3. ARE coil parameters

\begin{tabular}{ll}
\hline Number of coils & 96 \\
Coil configuration & D-shape \\
Conductor material & $13 / 16-i n .^{2}$ copper \\
Number of turns & 24 \\
Winding configuration & 2 pancakes, three-in-hand \\
Winding area & $8.25 \mathrm{ill} \times 12.38 \mathrm{cIII}$ \\
Mean perimeter & $3.74 \mathrm{~m}$ \\
Current density & $1505 \mathrm{~A} / \mathrm{cm}^{2}$ \\
lurreñt & $6408 \mathrm{~A}$ \\
Ampere-turns & 153,800 \\
Power per coil & $0.15 \mathrm{MW}$ \\
Total power & $14.7 \mathrm{MW}$ \\
Coolant flow rate (per coil) & $32.4 \mathrm{gpm}$ \\
Coolant temperature rise & $18^{\circ} \mathrm{C}$ \\
Voltage & $23.4 \mathrm{~V}$ \\
\hline
\end{tabular}

Support structure. The ARE coil support structure, which is fabricated entirely from stainless steel, is shown in Figs. 3.13 and 3.14. This support structure must be capable of adequately supporting the ARE coils for an ARE coil current either opposing or aiding the mirror coil current. The ARE coils are hinge mounted at three points around their periphery to rigid, cantilevered vertical support 


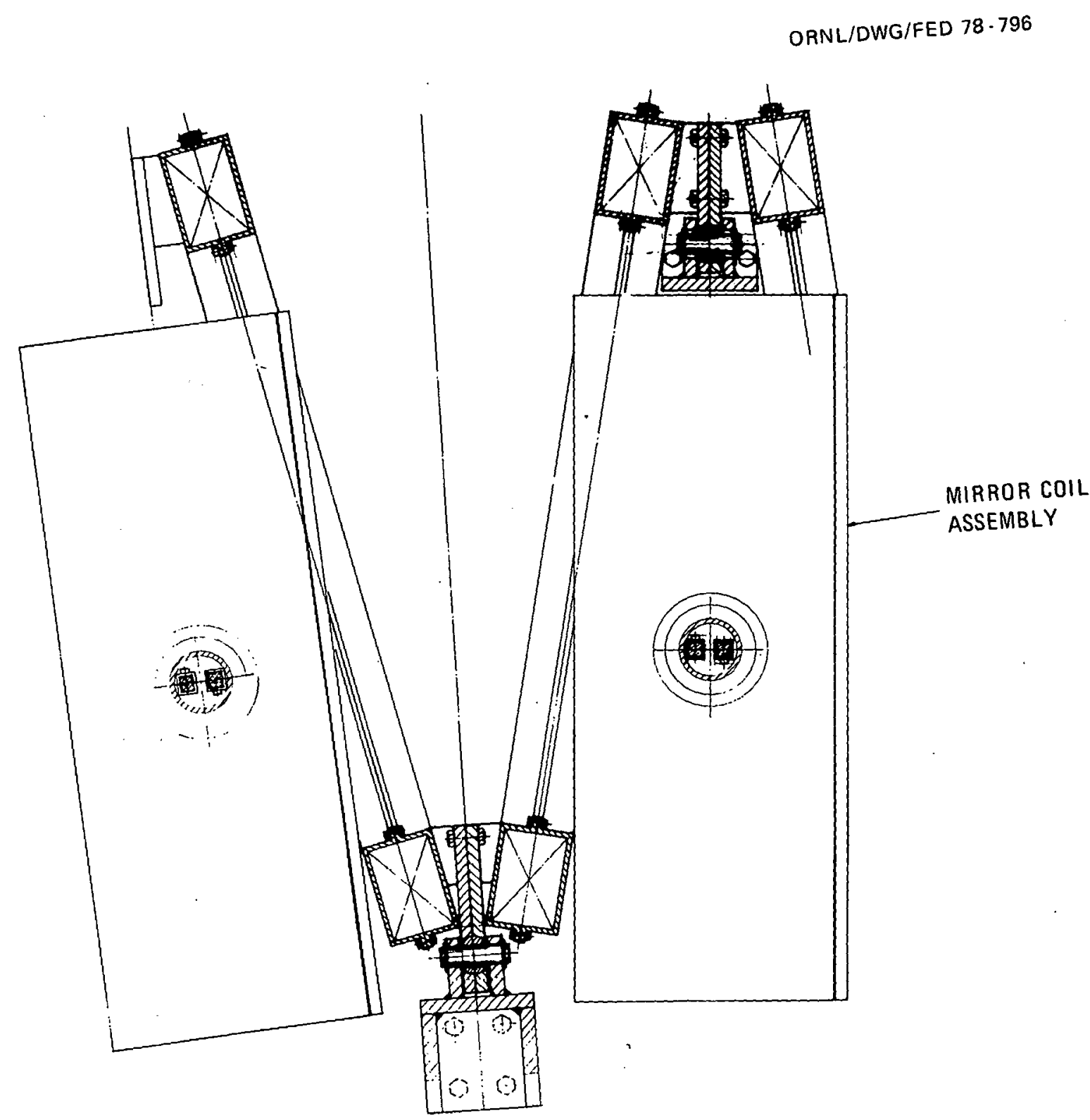

Fig. 3.13. Plan view of ARE coil restraint system. 


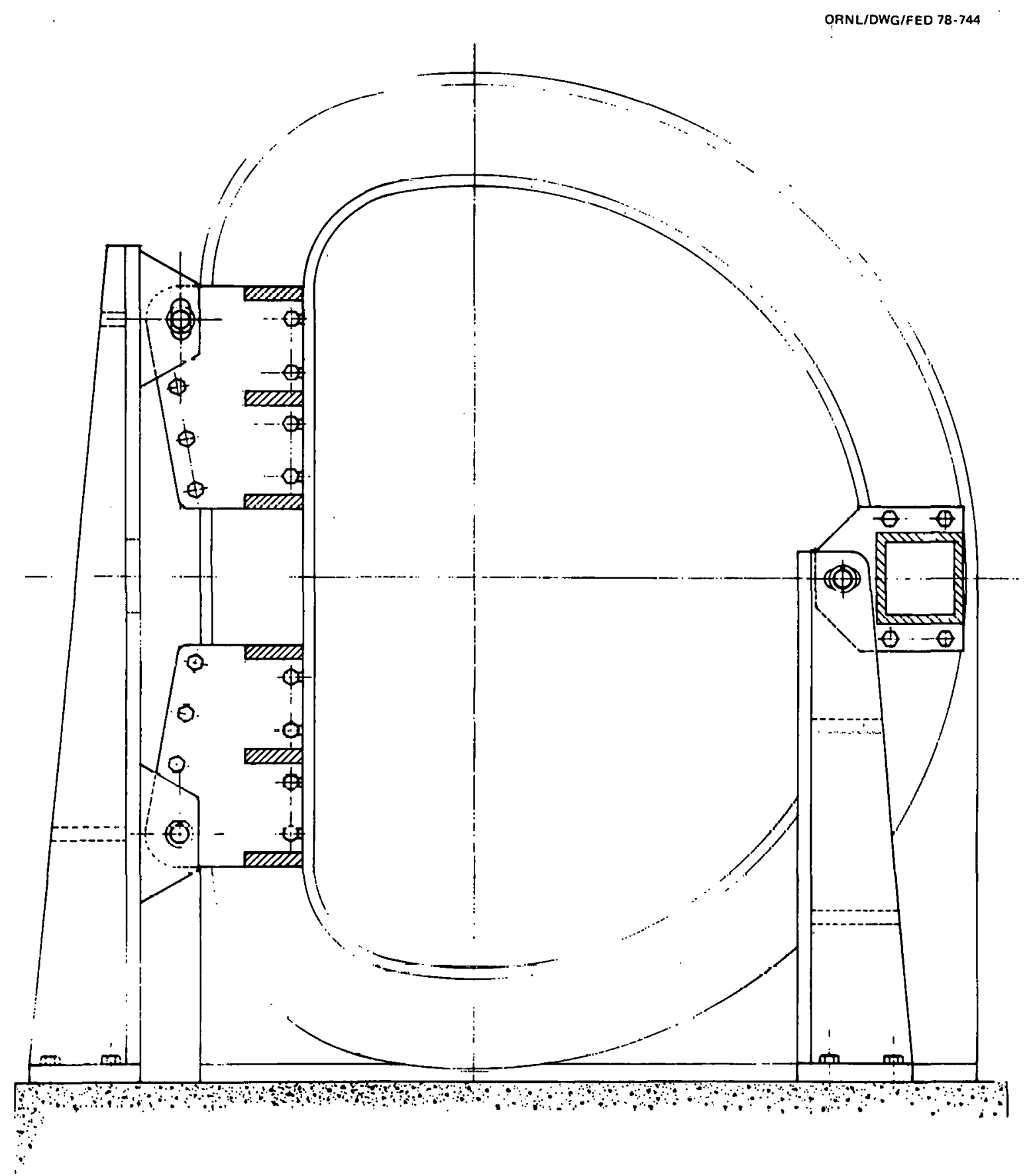

Fig. 3.14. Elevation of ARE coil restraint system. 
beams. The support beams are fastened to the concrete support ring with anchor bolts. Two hinge joints are located on the inner leg of the ARE coils, above and below the horizontal midplane, and another hinge joint is located on the outer coil leg at the horizontal midplane. The cantilevered support beams provide support for ARE coil gravity loads ( 800 1b per coil), centering forces (28,000 1b max per coil), and out-of-plane (fault) loads $(14,400$ lb max per coil). Out-of-plane forces are transferred through the support beams only during fault conditions. Details of the coil loading are presented in Sect. 3.7.2.3.

The two inner leg hinge joints are located between adjacent coils of an ARE coil set (the two ARE coils which encircle a cavity section). The hinge joints are formed by bolting together structural flanges which are welded to the side of each coil case on the inner leg. The structural flanges provide additional out-of-plane support to the coil inner legs, where the most severe out-of-plane loading is ericuuntered by the ARE coils during normal operation. The outer leg hinge joint is located between the coils of adjacent ARE coil sets. This hinge joint, like those on the inner leg, is formed by bolting together structural flanges which are welded to the ARE coil cases. Out-of-plane support for the coil outer leg is provided by the structural flange.

The ARE coils are mounted to the cantilevered vertical support beams with a pinned U-joint arrangement at each hinge location. Each hinge joint contains a ball joint (Fig. 3.13) which allows coil rotation in both radial and lateral directions. These are the only degrees of freedom allowed at the lower inner leg hinge joint. The upper inner leg hinge joint is slotted vertically to allow axial translation of the hinge point. The ARE coil centering forces are resisted entirely by these two inner leg hinge joints. The outer leg hinge joint is slotted horizontally to allow radial translation of the hinge point. Lateral translation is prevented at each hinge joint. This minimal restraint support system minimizes stresses in the ARE coils.

\subsubsection{Structural analysis}

Magnetic force analysis. Magnetic force calculations for the ARE coil-mirror coil system were preformed using the BARC8 computer code. BARC8 calculates magnetic forces by modeling the coils as monofilament current loops. Three cases were investigated:

case 1. ARE coil current opposing mirror coil current $\left(I_{A}=6.5 \% I_{M}\right)$,

Case II. ARE coil current aiding mirror coil current $\left(I_{A}=6.5 \% I_{M}\right)$,

Case III. ARE coil current opposing mirror coil current with one mirror coil faulted $\left(I_{A}=6.5 \% I_{M}\right)$.

The resulls uf lhe dilalysts are presented in lable 3.4 and Figs. 3.15 and 3.16 . Table 3.4 presents a summary of the resultant in-plane (centering force) and out-of-plane loads on both the mirror coils and ARE coils. As is evident from Table 3.4, reversal of the ARE coil current causes a change in the direction of the centering force on both the ARE coils and the mirror coils, which indicates the use of bidirectional suppurts. The centering force on the mirror coil is outward when the ARE coil current is in the same direction as the mirror coil current. It must also be noted that mirror coil faulting conditions are less severe on tie ARE coils than normal operating conditions. Figures 3.15 and 3.16 show the in-plane and out-of-plane load distributions around the periphery of the coils. The most ilipurtant point to be ascertained from these plots is that both the in-plane and out-of-plane loadings on the ARE coils are most severe at the straight inner coil leg.

Stress analysis. Preliminary analyses, sufficient to establish firmly the structural integrity of the EBT-II magnetic design, have been performed. Analyses were performed on both mirror coils and ARE coils, utilizing the magnetic load distributions presented in the preceding section.

The superconducting mirror coil stress analysis was performed by hand calculation assuming a thick wall ring loaded by uniform internal pressure. The loading used in the analysis corresponded to Case II 


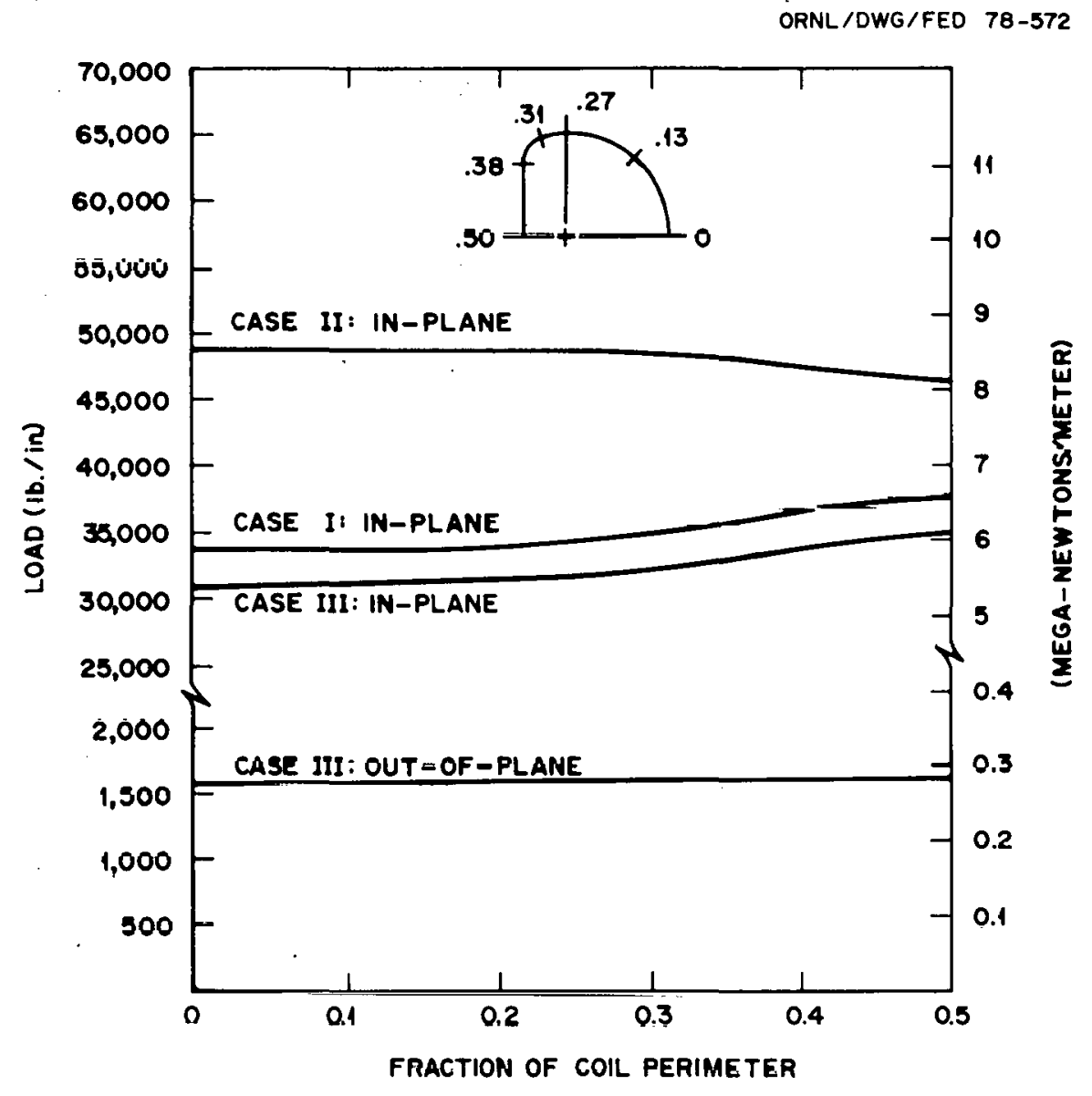

Fig. 3.15. Mirror coil magnet1c load distributiun. 

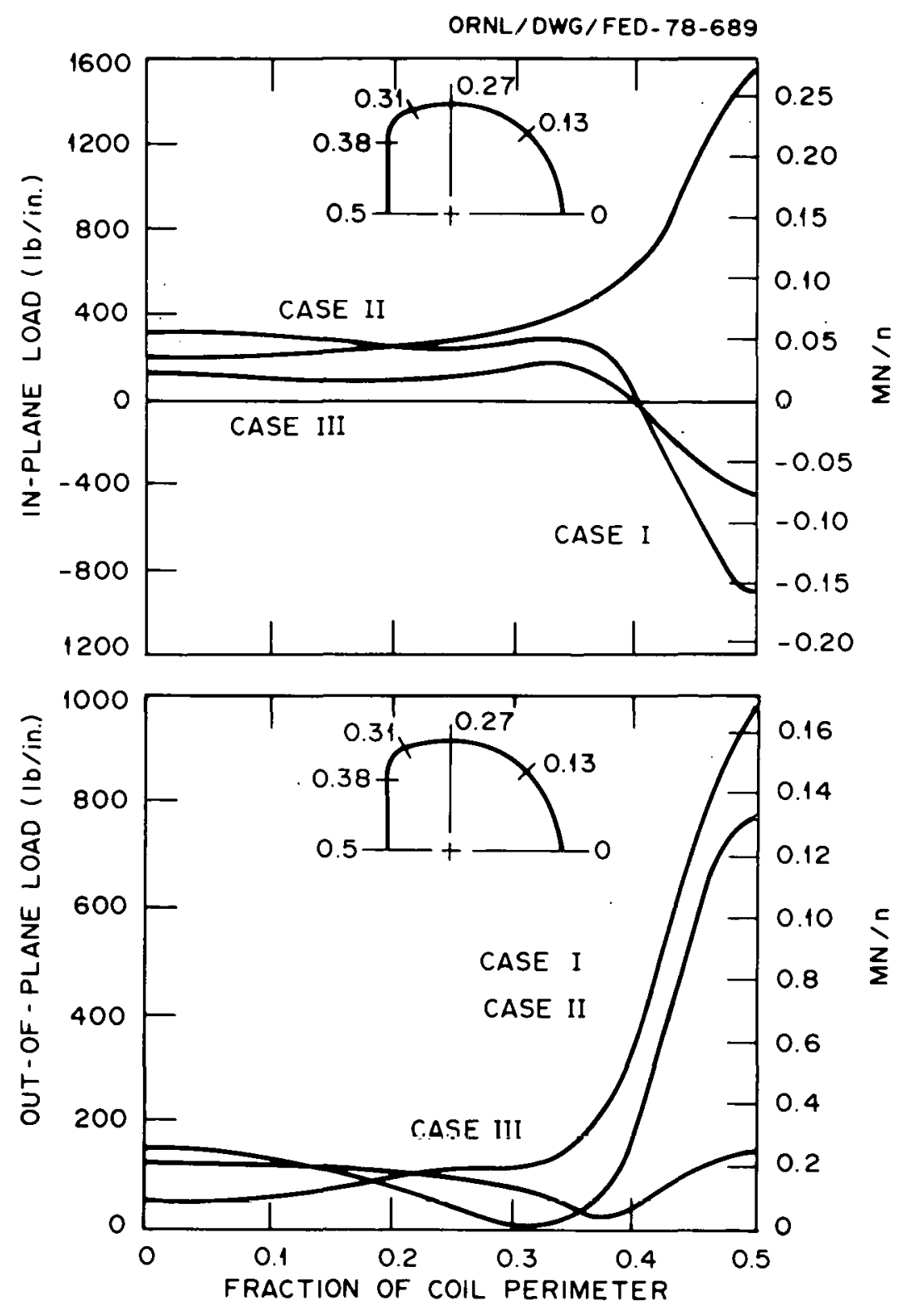

Fig. 3.16. ARE coil magnetic load distribution. 
in Fig. 3.15, and it was assumed that the coil winding was independent of the coil case (all load taken by winding). This conservative analysis showed the conductor to have a maximum hoop stress of 15,700 psi, which corresponds to a hoop strain of $0.087 \%$. This strain level is under the operating 1 imit for both $\mathrm{NbTi}$ and $\mathrm{Nb}_{3} \mathrm{Sn}$ superconductors.

Table 3.4. Summary of resultant magnetic forces

\begin{tabular}{lcccc}
\hline & \multicolumn{2}{c}{ Mirror coil } & \multicolumn{2}{c}{ ARE coil } \\
\cline { 2 - 5 } Case & $\begin{array}{c}\text { Centering force } \\
(1 \mathrm{~b})\end{array}$ & $\begin{array}{c}\text { Lateral force }^{b} \\
(1 \mathrm{~b})\end{array}$ & $\begin{array}{c}\text { Centering force }^{a} \\
(1 \mathrm{~b})\end{array}$ & Lateral force $^{b}$ \\
(1b)
\end{tabular}

Negative value denotes force away from device.

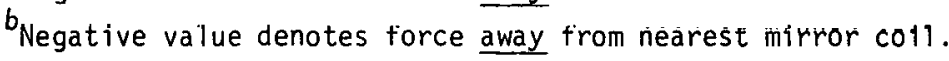

The ARE coil stress analysis was performed using SAP $V$, a three-dimensional, finite element computer program. The coil finite element geometry was represented by the section of coil encompassing the three hinge joint mount locations, as shown in Fig. 3.17. The stainless steel coil case, copper conductors, and epoxy interfaces were represented by 64 brick elements, 128 plate elements, and 29 boundary elements. The load conditions representing the most severe in-plane and out-of-plane combined loading (Case II, Fig. 3.16) were used in the analysis. The difference between the model shown in Fig. 3.17 and the actual design configuration is the simulation for the structural flanges on the side of the coil case. The model conservatively has no flanges, which results in higher stresses on the coil inner leg than actually exist. The deflected shape of the ARE coil model was calculated and is shown in Fig. 3.18. Coil support reaction forces and coil peripheral stress distributions were also calculated and are shown in Figs. $3.19,3.20$, and 3.21 .

As can be seen from Figs. 3.20 and 3.21, the maximum stresses in the coil occur at the coil inner leg. The 3850-psi hoop stress in the copper windings and the 7460-psi hoop stress in the stainless steel case are both well below their respective limits. The maximum shear stress of 1170 psi in the epoxy layer between conductors is below the 1500-psi shear allowable for epoxy. (If the windings were not contained in a structural case, this shear limit would be exceeded and the windings would delaminate.) As noted, the actual stresses in the coil inner leg will actually be less than those indicated by the analysis, due to the presence of the inner leg structural mounting flanges.

No attempt was made in this study to generate a restraint system which was structurally compatible with the support loads shown in Fig. 3.19 .

\subsubsection{Test and procurement}

Objectives. The objectives of the test and procurement activity are to obtain reliable, costeffective magnets that have been thoroughly tested for EBT-II and to deliver them to the EBT-II site. The activity is divided into a prototype program, an acquisition program, and an acceptance program.

The EBT devices require a very high degree of symmetry in the vacuum fields, so the mirror coils should all be identical. Also, management of a program to build a large number of different magnets would be costly and time-consuming. 


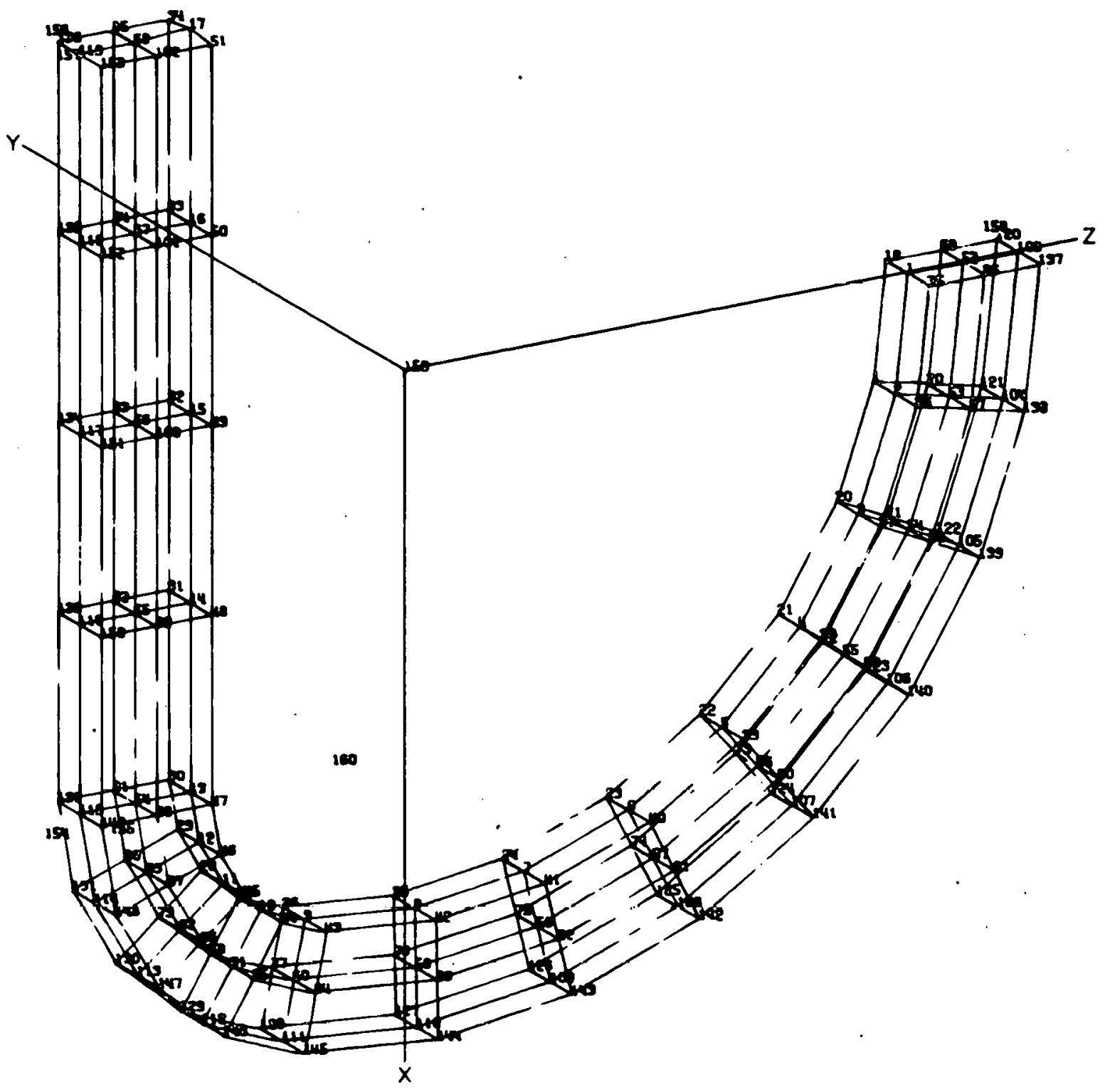

Fig. 3.17. Finite element model of ARE coil. 


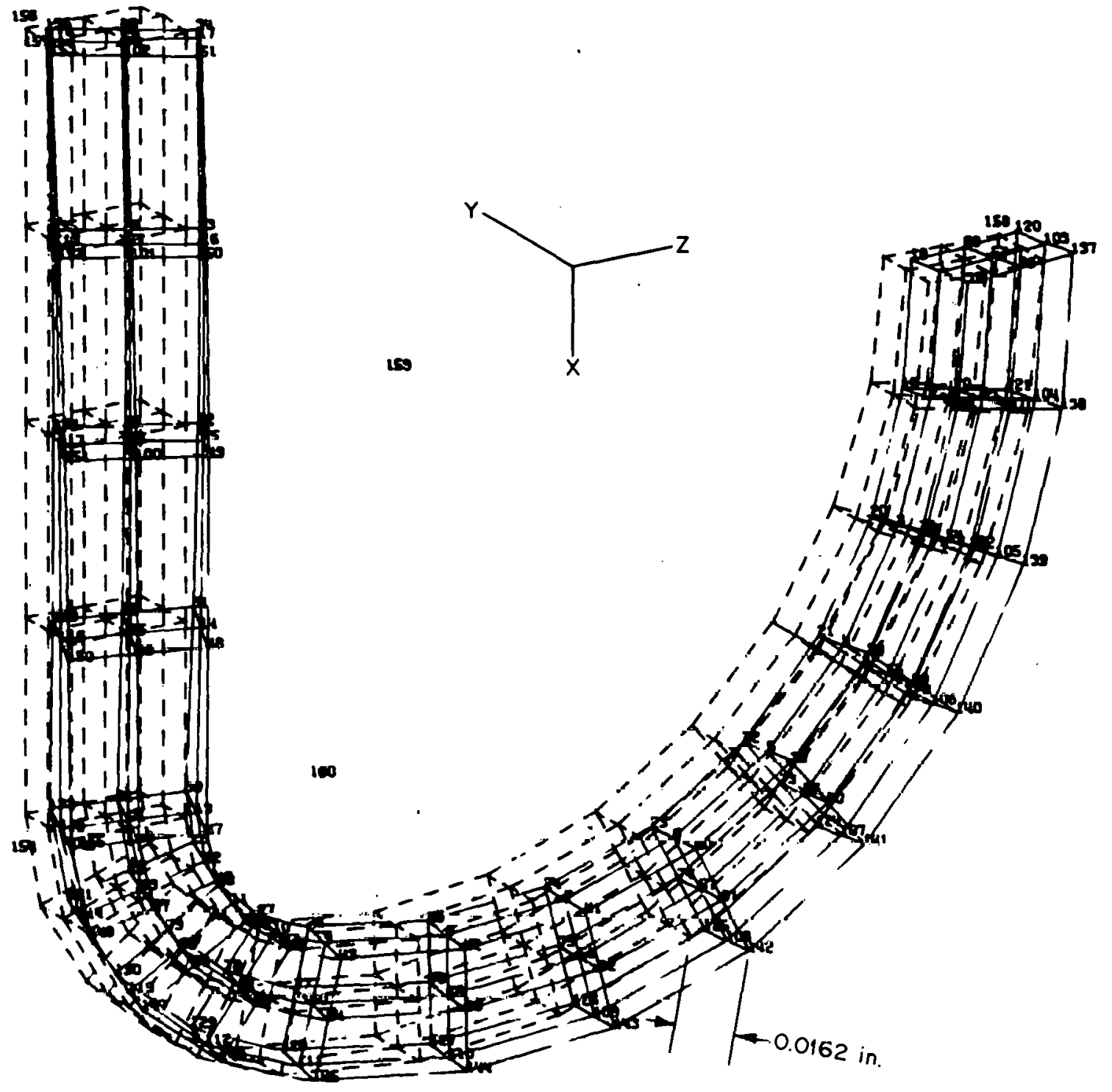

Fig. 3.18. Deflected ARE coil model. 
45

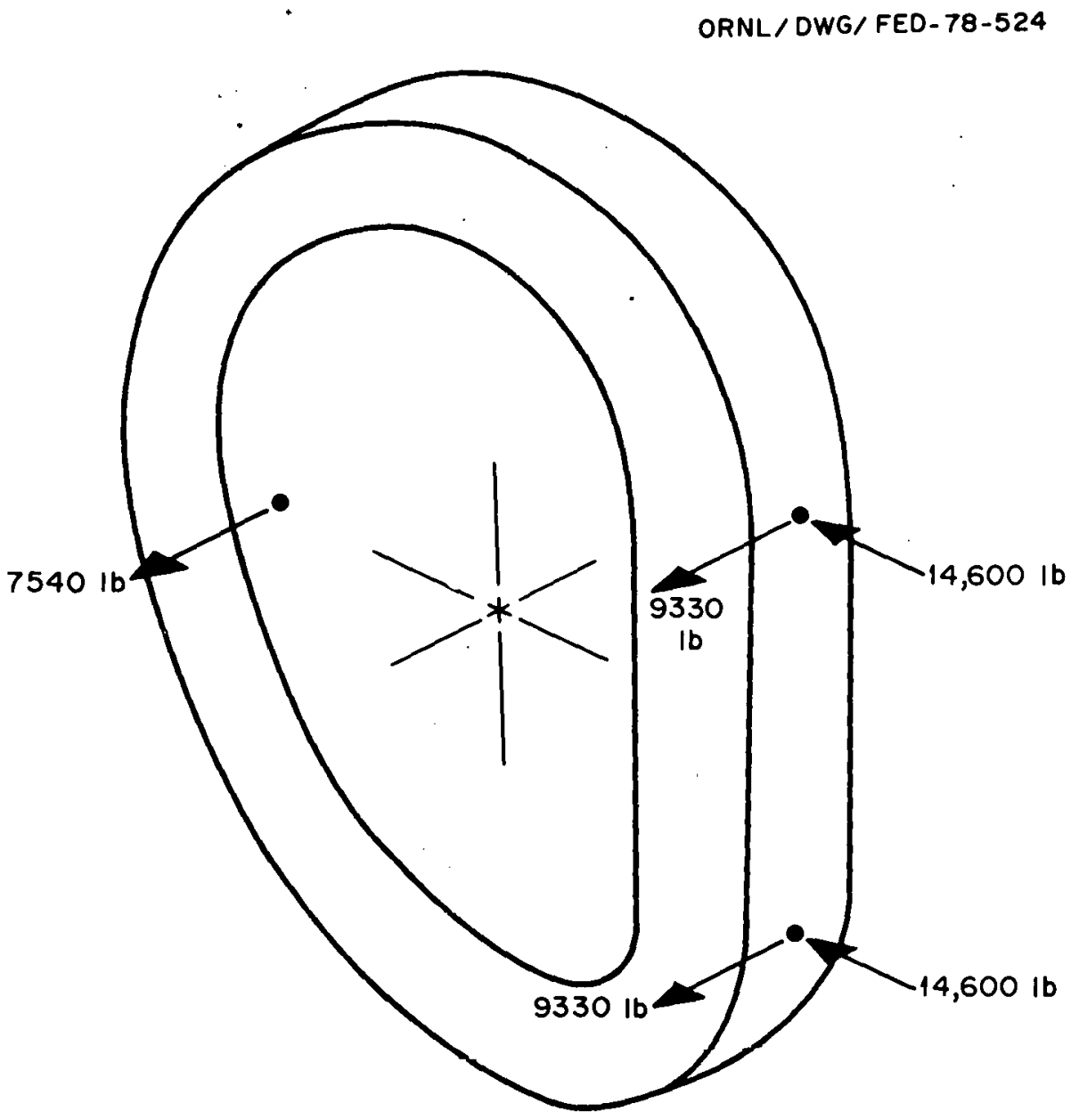

Fig. 3.19. Reactions at ARE coil support locations. 


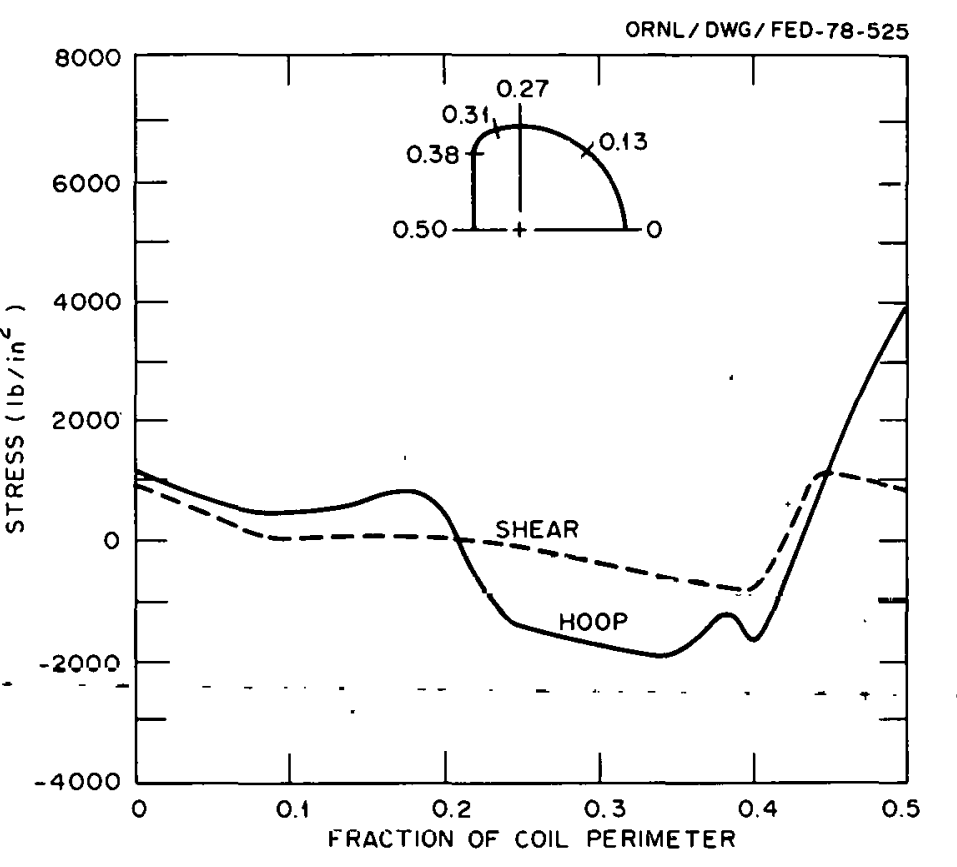

Fig. 3.20. ARE coil winding stress distribution.

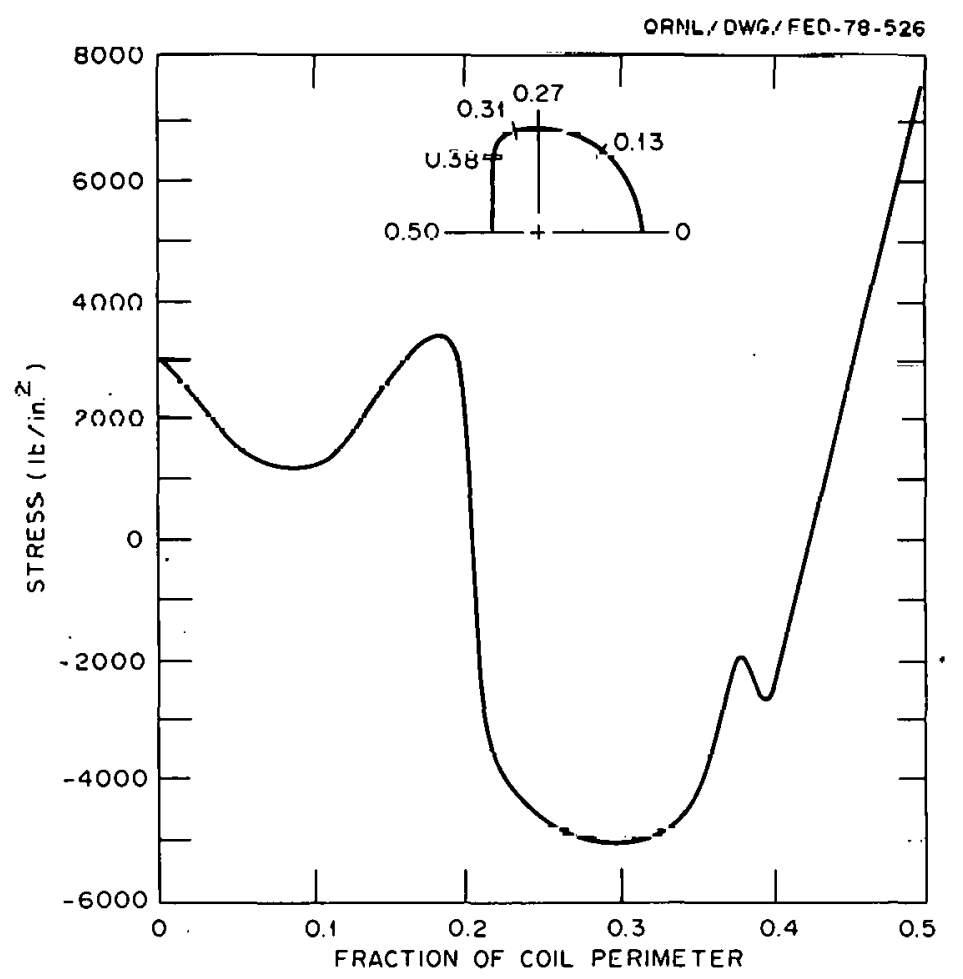

Fig. 3.21. ARE coil case stress distribution. 
The time schedule for manufacture and delivery of the mirror coils will be too short for one manufacturer to deliver the 55 to $60 \mathrm{NbTi}$ magnets, so two manufacturers will be used. Work may be shifted from one coil manufacturer to another if one's schedule starts to slip. This will help ensure competitive delivery and cost bidding. More than two manufacturers would be harder and more costly to manage.

The decision to have one magnet design and use two manufacturers implies that at least one of the manufacturers will be making someone else's design. Therefore, the design should be well defined and thoroughly tested and should not contain proprietary features supplied by only one manufacturer. The prototype program will supply a well-defined, tested design to be bid on by the superconducting magnet manufacturers, and the acquisition program will put the contracts out for bid and oversee the manufacturing of the complete set of mirror coils. The acceptance program will then test each magnet as it is completed and deliver it to the. EBT-II site.

There are two other jobs in addition to the superconducting mirror coils: the dewars and the ARE coils. These jobs will be bid separately. Since it has not been decided whether the ARE coils will be superconducting or resistive, they present a dual problem. If they are superconducting, then two manufacturers will be used, as in the mirror coil case. If they are resistive (the most likely case), there will probably be only one magnet maker since the ARE coils are smaller and simpler to make and will not therefore be a pacing item.

Subtask description. The three subtasks - the prototype program, the acquisition program, and the acceptance program - are detailed below.

1. Prototype program: The purpose of the prototype program is to specify the design of the magnets and dewars, to construct the prototypes, and to test the prototypes to ensure that they meet the EBT-II specifications. The data generated will be used to define the acceptance tests for each magnet and dewar.

The design phase of the prototype program must deal with the overall interface of the magnet system with EBT-II, the design of the tests and test facility, and the design of the prototype magnets. When the magnet design is fixed, then the tests and test facility can be designed. The test stand will use three or possibly four mirror coils. (The final decision has not been made yet.) The test program sequence, the design of the test facility, and the tests will be done at ORNL.

It has not been decided yet whether to buy magnet designs from industry and incorporate features of those designs into an ORNL design or to use an ORNL design. In either case, ORNL will be involved in the design process, and manufacturers other than the designer can bid on the final magnet construction contract.

Since the procedure for obtaining the prototype coil is a departure from the document submitted in 1977, a few words on the reason for the change are in order. Time will be saved in writing specifications, bidding, and selecting a vendor. Constructing the prototypes in-house ensures complete knowledge of all the details and ensures that the manufacturing details are provided equally to all prospective vendors of one system. Also, the prospective vendors of the magnets for the system are bidding on an equal basis with no one having had an advantage over any other through prototype construction.

Prototype dewars, superconducting mirror coils, and ARE coils will be made at ORNL. This will ensure that the manufacturers have a tested design to build and that all problems are well known. It will also give ORNL maximum flexibility in designing the instrumentation and diagnostics for the prototype tests.

The prototype tests will be proof tests and will provide performance data. The dewar test will provide data on heat leaks, cooldown stresses, and coil movement during cooldown. The field accuracy tests on the mirror coils will be made with and without the dewar to establish the source of potential 
trouble. The mirror coils will be operated with and without the ARE coils to prove superconducting operation and mechanical integrity. The ARE coils will also require field accuracy tests. Then the whole system will have to be tested. Finally, the prolection circuits will be proof tested and each coil will undergo a quench without the discharging of all the other coils. The test facility will be used again in the acceptance test phase of the program.

There are two major candidates for the mirror coil design: potted and ventilated coils. In ventilated coils, liquid helium is able to percolate in intimate contact with the windings; usually $50 \%$ are not covered by insulation. The ventilated coils can remove a larger steady-state $x$-ray heat load than the potted coils, but there is considerable industrial experience with potted coils of the type required. One design of each type will be tested.

2. Acquisition program: The mirror coils, ARE coils, and dewars for the EBT-II system will be procured from industry. The acquisition program will combine the prototype design data, manufacturing data, and test data into bid packages and a quality assurance plan; oversee the bidding process; select the manufacturers; and ensure that time schedules, cost estimates, and quality assurance objectives are met.

3. Acceptance program: The prototype test facility will be used to test each supcrconducting coil before it is delivered to the EBT-II site for installation. The acceptance test criteria will be established in the prototype program.

\subsubsection{Vacuunt Pumping Systems}

The EBT-II primary vacuum system performs two functions:

1. It must initially reduce the pressure in the torus and remove the high $Z$ impurity gases from surface outgassing during operation.

2. It must handle the additional gas load when operating with neutral beam injection. In addition, the beam dump pumps must remove all of the injected neutral hydrogen which passes through the plasma and is not trapped.

In both cases, pumping is accomplished by cryocondensing the gas on liquid heliull couled cryopanels operating at 4.2-5K. The cryocondensing pumps will have a liquid nitrogen cooled chevron baffle and a liquid helium cooled cryocondensing surface to pump hydrogen and air. The liquid nitrogen cooled chevrons will trap water and carbon dioxide.

The rough vacuum system performs three functions:

1. It reduces the pressure in the torus and cryopumps at each startup to $10^{-5}$ torr (this will normally be done by the zeolite cryopump).

2. It pumps down the helium dewars surrounding the superconducting coils prior to cooldown.

3. It regenerates the cavity and beam dump cryopanels (this will be done when the experiment is not in operation).

The design criteria are as follows.

1. The plasma vacuum vessel is a nonbaked aluminum vessel with Viton 0 -ring seals.

2. The ultimate pressure will be $2.5 \times 10^{-5}$, torr, including hydrogen.

3. Hydrogen for pressure regulation will be admitted through a piezoelectric valve which is controlled by an ionization gage pressure regulator. Normal pressures will be in the range of $10^{-5}$ torr.

4. Pumpdown will require no more than several hours.

5. Base pressure in the superconducting coil vacuum dewars will be $10^{-5}$ torr. 
6. The cryopumps on the cavities and beam dumps will be regenerated when the device is not operating.

7. Backstreaming from neutral beam injection and dump ports will be 1 imited to 0.5 torr -1 iters/sec, less if possible.

Characteristics of the proposed system are listed in Table 3.5. A schematic of the system is shown in Fig. 3.22.

Table 3.5. Vacuum system characteristics

\begin{tabular}{|c|c|}
\hline Torus volume & $4.5 \mathrm{~m}^{3}$ \\
\hline Torus surface area & $44.4 \mathrm{~m}^{2}$ \\
\hline Outgassing rate & 0.044 torr-liter $/ \mathrm{sec}$ \\
\hline $\begin{array}{l}\text { Net pumping speed (hydrogen) per } \\
\text { port }\end{array}$ & 40501 iter/sec \\
\hline $\begin{array}{l}\text { Total pumping speed (hydrogen) for } \\
48 \text { ports }\end{array}$ & $195,0001 \mathrm{iter} / \mathrm{sec}$ \\
\hline Base pressure (hydrogen) & $2 \times 10^{-7}$ torr \\
\hline Primary pumping system & $\begin{array}{l}4825,000-1 \text { iter/sec hydrogen cryocon- } \\
\text { densing pumps }\end{array}$ \\
\hline \multirow[t]{3}{*}{ Roughing system } & $\begin{array}{l}\text { One } 84-1 \text { iter/sec liquid nitrogen cooled } \\
\text { cryosorption pump }\end{array}$ \\
\hline & Two 1500-1iter/sec turbomolecular pumps \\
\hline & One 50-cfm mechanical pump \\
\hline
\end{tabular}

\subsubsection{Microwave Heating Systems}

Twelve 200-kW gyrotrons will be located within the torus enclosure to provide power for plasma heating and stabilization. Each tube will be mounted on the lid of an oil tank, with the high voltage gun completely immersed in an oil bath. The tanks will be spaced $30^{\circ}$ apart around the perimeter of the machine. Eight of the gyrotrons, operating at a frequency of $120 \mathrm{GHz}$, will generate $1.6 \mathrm{MW}$ of bulk heating power while the remaining four tubes will be used to supply a total of $800 \mathrm{~kW}$ of profile heating at about $90 \mathrm{GHz}$. (The exact frequency has not yet been determined: it is of this order or less.) Each quarter of the torus will be powered by a single 90-GHz tube located between two 120-GHz tubes so that a symmetric array of power devices is installed around the machine. This layout will simplify the design of a suitable microwave power distribution system and will facilitate the integration of additional power sources at some later date, if they are required.

\subsubsection{Gyrotron development}

A gyrotron (cyclotron resonance maser) is currently being developed by Varian Associates to provide bulk heating on EBT-S at $28 \mathrm{GHz}$ and $200-\mathrm{kW} \mathrm{cW}$ power. This device will be the prototype for the higher frequency tubes required for bulk $(120 \mathrm{GHz})$ and profile $(90 \mathrm{GHz})$ heating on EBT-II, which will be developed by scaling the EBT-S tube in frequency. An oscillator rather than an amplifier is proposed in this design report because this approach represents the path of lowest technical risk. 
ORNL/DWG/FED $78-749$

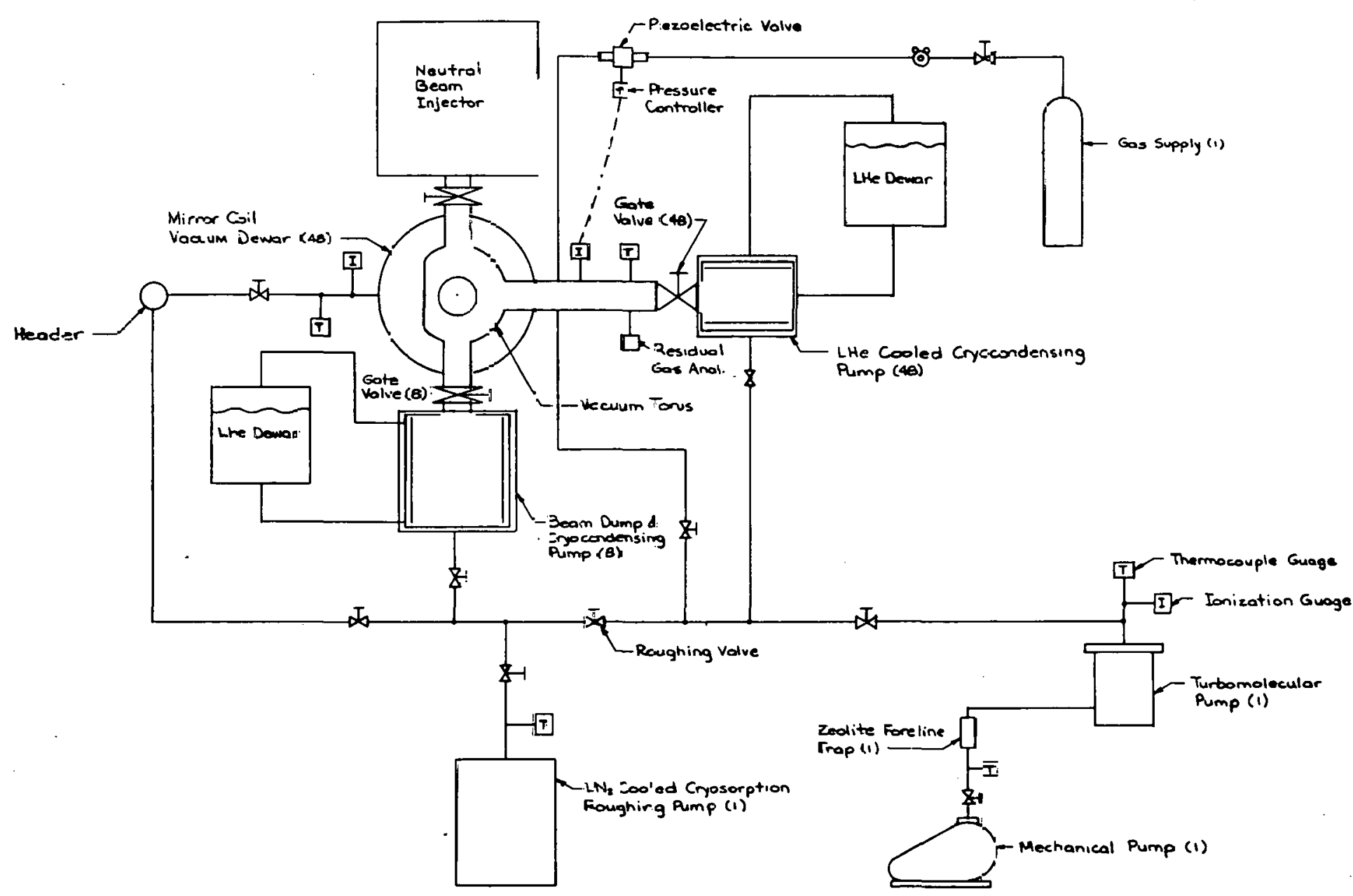

Fĭg. 3.22. Vecul'm system flow ciagram. 
Research in this category of ultrahigh frequency microwave generation has been stimulated by the recognition of the interesting characteristics inherent in this specific type of microwave tube:

1. output power is generated in circular electric modes which can be propagated in oversized cylindrical waveguides (typically ten or more wavelengths in diameter) with the very low power losses characteristic of this mode family, and

2. typical tube dimensions can be greater than one wavelength because the beam-microwave coupling is fundamentally different from that employed in conventional microwave tubes (klystrons, traveling wave tubes, etc.).

Since the operating frequency of a gyrotron is directly proportional to the focusing magnet axial field strength, an increase in frequency by a factor of three to five will require a three- to fivefold increase in the on-axis magnetic field. Therefore, the $120-\mathrm{GHz}$ and $90-\mathrm{GHz}$ tubes will require superconducting magnets which will be cooled by the torus cryogenic system. 0ther tube cooling requirements (except those for the electron gun and power supply interface components) will be met by the demineralized water system connected to the gyrotrons by a series of manifolds that will supply u560 gpm per tube. 0il-to-air heat exchangers, located outside the torus enclosure, will cool the electron guns and other oil tank components.

\subsubsection{Distribution system}

A power dividing system will be developed, based on quasi-optical techniques, to distribute the microwave output from each gyrotron to an integral number of cavities. Power will be transmitted in the low loss circular electric modes.

Profile heating at $90 \mathrm{GHz}$ will be supplied by four tubes, each of which will feed 12 cavities. Bulk heating power at $120 \mathrm{GHz}$ will be supplied by the remaining eight tubes that will be coupled to six cavities each. Bulk and profile heating power will be combined and injected into each cavity at the lower $45^{\circ}$ port with a single waveguide link.

A layout of the proposed power distribution system shows that both three-way and two-way power dividers must be developed to operate at both 90 and $120 \mathrm{GHz}$. The presence of the EBT-S tube at 0ak Ridge will allow experimental verification of various quasi-optical power divider designs at $28 \mathrm{GHz}$. Also, since the EBT-II tubes will operate in the same circular electric modes, designs developed and tested at $28 \mathrm{GHz}$ with the EBT-S tube can be directly scaled to the higher frequencies required for EBT-II.

High power windows will be required to mechanically isolate the plasma vacuum vessel from the waveguide system. These windows will be essentially the same as those developed to seal the vacuum at the gyrotron waveguide output and are to be developed along with the high frequency tubes.

\subsubsection{Vacuum and neutral beam systems isolation}

It will be necessary to shield the cryogenic vacuum systems and neutral beam source region from scattered microwave energy to prevent evolution of condensed gases on the cryopanels and to eliminate the possibility of interference with neutral beam production and propagation. This will be accomplished in the torus vacuum system by installing parabolic reflectors in each of the 48 cryopump ducts. These mirrors will be sized to allow the vacuum conductance required to maintain system pressure with the installed pumping speed and neutral beam gas feed rates. Water cooled annular dielectric rings will be 
designed to absorb any microwave power that is scattered past the reflectors. Analytical calculations have demonstrated that reflectors of the required size will not significantly degrade the vacuum system conductance.

Isolation of the neutral beam dump cryopanels and source region from the scattered microwave energy will be accomplished through the use of water cooled power absorbing annular rings similar to those used in the torus system. Reflectors obviously cannot be used in these systems, but as the ratio of their entrance areas to the total torus internal wall surface area is very small, the microwave power loss will be negligible.

Detailed design of both the reflectors and water cooled dielectric loads will require an investigation of the properties of the relevant materials at the very high frequencies proposed, as well as a good estimate of the power absorbing efficiency of the plasma itself. The gyrotron development program will generate the required materials property data, and experience with EBT-S should allow a reasonable estimate of the microwave energy absorption efficiency of the plasma for mechanical design purposes.

\subsubsection{Neutral Beam Heating Systems}

The addition of neutral beam heating to EBT-II constitutes Phase 2 of the experiment; the beam lines will not be installed until most of the Phase 1 experiments are complete. Therefore, the design criteria for the neutral beams do not need to be as firm as for the other systems at this time. tvery effort has been made to plan for a neutral beam injection system which will allow significant plasma ion energy enhancement at a reasonable cost, but the details are left somewhat vague since the confinement results obtained from Phase 1 experiments will undoubtedly influence the design parameters of the neutral beam system.

\section{1 .5 .1 Summary of design criteria}

1. Neutral beam injection will be perpendicular to the plasma (vertically down) at the middle of the vacuum cavity (spool piece).

2. One megawatt of extracted beam power will be supplied initially by four beam lines.

3. The neutral particles will have $20-k e V$ energy.

4. The beam 11 nes will operate $\mathrm{cw}$.

5. A beam dump capable of handling full power will be located $180^{\circ}$ from the injection port. All particles hitting the beam dump will be pumped from the system.

6. Maximum total gas streaming rates from all beam lines and dumps will be 0.5 torr-liters/sec.

\subsubsection{Operating characteristics}

Each beam line will operate at $250 \mathrm{~kW} \mathrm{dc}(20 \mathrm{kV}, 0-12.5 \mathrm{~A})$ for a total of $1 \mathrm{MW}$ of installed or extracted power. Efficiencies of $50 \%$ to $75 \%$ are expected for these low power beams, so a total. of $2750 \mathrm{~kW}$ of neutral beam power can impinge on the plasma from four beam lines. rour beam 1 ines were chosen on the basis of realizable dc operation.

Not all of the incident power is absorbed by the plasma, and the goal for this design study is to provide sufficient incident power so that $300 \mathrm{~kW}$ can be absorbed by the toroidal plasma for Phase 2

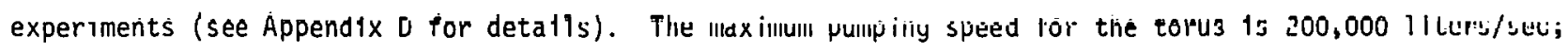
this steady-state gas flow determines the wall pressure. Detailed confinement studies are necessary to determine the operating pressure required to maintain the plasma during neutral injection. 
Vertical injection from above the torus appears to be the best injection scheme for the following reasons. The canted, D-shaped ARE coil design limits access to the vacuum cavity along the inner radius. Locating a port for the beam dump $180^{\circ}$ from the injection port can be obtained only with vertical injection. The neutral beam source is a high maintenance item and needs to be located on the roof. Aligning the source to assure maximum power impinging on the plasma can be accomplished with this scheme.

Two 15-cm-diam ports on opposite sides of the spool piece serve as the injection port and the beam dump port. The beam lines will be designed for a focal point which allows the entire beam to pass through the lower port onto a beam dump.

\subsubsection{Component description}

Gas cell neutralizing efficiency and beam divergence are unknown and introduce uncertainties in the design of the deflecting magnet and ion dump. The cold gas flow from the source is an uncertainty which affects the sizing of the cryopanels in the same way. These unknowns make it difficult to accurately size the system.

With the same beam optics at $20 \mathrm{keV}$ as at $40 \mathrm{keV}$, the beam line length from the source electrodes to the focal point will be approximately $3 \mathrm{~m}$.

Neutral beam source. The neutral beam source will be a modified duopIGatron ion source operating at $20 \mathrm{kV}$ and $12.5 \mathrm{~A}$. It will produce $20 \mathrm{-keV}$ ions continuously. The source will be shielded from stray magnetic fields and will be located on the enclosure roof at a workable level. It will be isolated from the vacuum enclosure with a valve and steering mechanism.

Vacuum enclosure. The vacuum enclosure is the central beam line component and is $\sim 1 \times 1.8 \times 1.6 \mathrm{~m}$. The gas cell is $230 \mathrm{~cm}$ long and will be capable of $90 \%$ neutralization. Large cryopanels will be positioned to either side of the cell to maintain the proper pressure. The bending magnet and ion dump will be 50-70 cm long and will be sized to handle 20-keV ions. Water cooled copper plates will occupy a few centimeters of beam line length to define the beam. Large cryopanels to maintain low pressure in the drift tube and a flat swirl tube calorimeter will occupy the end of the box.

Drift tube and isolation valve. The drift tube and isolation valve will be approximately $35 \mathrm{~cm}$ long. The drift tube will be water cooled, with a microwave absorbing inner surface to minimize microwave penetration into the beam line.

Beam dump. The beam dump will dissipate the energy of all beam particles not interacting with the plasma. It will also have sufficient pumping for all particles entering the dump. Each dump will be designed for $12.5 \mathrm{~A}$ of particles. To pump all of these particles will require approximately $6 \mathrm{~m}^{2}$ of surface area, which can be accomplished with four $1 \times 1.5-\mathrm{m}$ cryopanels. The beam dump will consist of a $35-\mathrm{cm}-$ long, 15-cm-diam tube that will bolt to the lower $15-\mathrm{cm}$ port and extend beyond the bottom of the ARE coils. This tube will be connected to a conical transition tube which extends through the concrete pad. The cone shape reduces beam interaction with the wall. The dimensions of the final tube extending to the beam dump enclosure will be determined from the beam divergence. The enclosure will contain the cryopanels and the dump target and will be $1.5 \mathrm{~m}$ wide, $1.5 \mathrm{~m}$ long, and $1 \mathrm{~m}$ high. This configuration has been chosen for maximum cryopanel surface in a minimum volume and good vacuum conductance from the beam target to the cryopanel surfaces. The target will be a water cooled copper plate positioned at the bottom of the enclosure and designed to handle about $200 \mathrm{~kW}$ of incident energy. The beam dump enclosure will rest on a steel support frame. 


\subsubsection{Electrical Systems}

The electrical loads for EBT-II will consist of dc power supplies for the gyrotron beams and gun anodes, for the toroidal field coils and gyrotron magnets, and for the neutral beam injectors; motorgenerator sets for the ARE coils; primary power for the helium refrigerator compressors; and normal building and auxiliary services for the experiment area, control room, and microwave power supply building. The electrical power load demand for EBT-II is expected to be 31 MVA. The estimated loads are shown in Table 3.6 .

Table 3.6. Estimated loads

\begin{tabular}{|c|c|c|c|}
\hline S.ysleII & $\underset{k \cup \mathcal{K}}{\text { Connected }}$ & $\begin{array}{l}\text { Demand } \\
\text { factor }\end{array}$ & $\begin{array}{c}\text { Demand } \\
\text { k.VA }\end{array}$ \\
\hline \multicolumn{4}{|l|}{ Gyrotren power supplics } \\
\hline $\begin{array}{l}\text { Beam power } \\
\text { Gun anode }\end{array}$ & $\begin{array}{r}12,000 \\
500\end{array}$ & $\begin{array}{l}1.0 \\
0.5\end{array}$ & $\begin{array}{r}12,000 \\
250\end{array}$ \\
\hline Toroidal field coil power supplies & 500 & 0.2 & 100 \\
\hline Gyrotron magnet power supplies & $500^{\circ}$ & 0.2 & 100 \\
\hline \multicolumn{4}{|l|}{ Neutral beam power supplies } \\
\hline $\begin{array}{l}\text { Accel } \\
\text { Snurre }\end{array}$ & $\begin{array}{r}1,000 \\
200\end{array}$ & $\begin{array}{l}0.5 \\
1.0\end{array}$ & $\begin{array}{l}500 \\
200\end{array}$ \\
\hline ARE coil motor-generator sets & 16,000 & 0.9 & 14,800 \\
\hline Helium refrigerator & 2,500 & 1.0 & 2,500 \\
\hline Control room & 100 & 1.0 & 100 \\
\hline Lighting & 50 & $i .0$ & 50 \\
\hline Auxiliary services & 400 & 0.25 & 100 \\
\hline Total & $\overline{33,75 n}$ & & $\overline{30.700}$ \\
\hline
\end{tabular}

\subsubsection{Primary power distribution $(13.8 \mathrm{kV})$}

Electrical power for EBT-II will be supplied from an existing 50-MVA, 161- to 13.8-kV transformer. Two 13.8-kV power circuit breakers wili be added to the existing switchyear in Bldy. 9201-2. Ohe of these circuit breakers will be used for control and protection of a feeder for the gyrotron power supplies. The other circuit breaker will be used to supply four unit substations, as described in Sect. 3.1.6.2. Metal clad feeder bus, rated at $2000 \mathrm{~A}$ and $15 \mathrm{kV}$, wili be used to extend the main bus from the existing switchgear to the EBT-II circuit breaker. The feeder to the EBT-II power supply building will consist of ethylene-propylene-rubber insulated 15-kV shielded cable in rigid conduit. The power distribution one-line schematic is shown in Fig. 3.23.

\subsubsection{Secondary power distribution (2.4 kV and $480 \mathrm{~V})$}

The helium refrigerator system will be supplied by a $2500 \mathrm{kVA}, 13.8$ - to 2.4-kV 3-phase unit substation. The unit substation will consist of a fusible 15-kV disconnect switch, a 13.8- to 2.4-kV, 3-phase oil filled transformer, and metal clad switchgear.

Low voltage power required by the toroidal field supplies, gyrotron magnet supplies, gun anode supplies, neutral beam power supplies, and auxiliary equipment will be provided by three 2500-kVA, 
ORNL/DWG/FED $78-756$

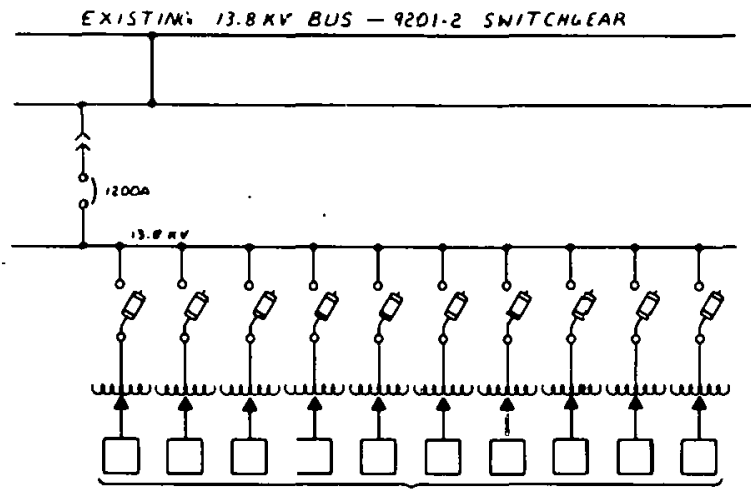

GYBOALYSTRON POWER SURPLITS
EXTENCEO $13.8 \mathrm{KV}$ BUS

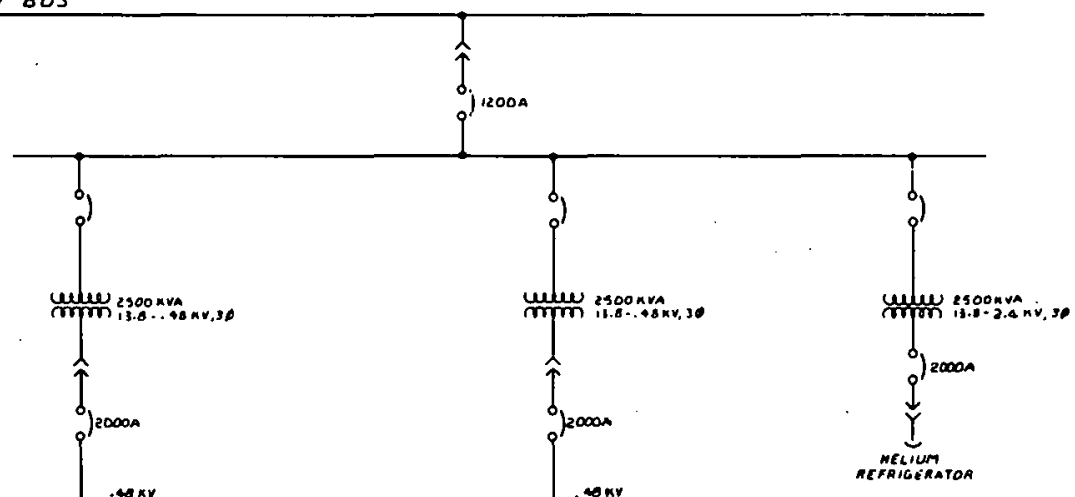

ज

Fig. 3.23. Switch gear diagram. 
13.8- to $0.48-k V, 3$-phase unit substations. Power to these loads will be transmitted by $600-V$ cable in rigid conduits to motor control centers and plug-in busway. This power distribution is shown in Fig. 3.23.

\subsubsection{DC power supplies}

Special purpose dc power supplies will be required for EBT-II in the following categories: high voltage power supplies for the gyrotron beam and gun anode, high voltage power supplies for the neutral beam accel grid and isolated power for the source, and high current power supplies for toroidal field coils and gyrotron magnets.

High voltage power supplies will be used to power 12 microwave generators to be used for bulk and profile heating on EBT-II. These power supplies will consist of two stacked, variable voltage, regulated power supplies. Each beam supply wili have a nominal rating of $100 \mathrm{kV}$ at $10 \mathrm{~A}$ and each gun anode supply will be nominally rated at $40 \mathrm{kV}$ at $1 \mathrm{~A}$. A one-line scllellatic of the gyrotion power supply ts shown in Fig. 3.24. The design and configuration of these power supplies will be based on the prototype power supply developed for EBT-S. An equipment arrangement of 12 gyrotron power supplies for EBT-II is shown in Fig. 3.25 .

The four accel power supplies for the neutral beam injectors will have a nominal rating of $20 \mathrm{kV}$ at $12.5 \mathrm{~A}$. These supplies will be variable voltage and wili include a modulating regulator at the output of the supplies. A one-line diagram of a typical neutral beam accel power supply for this application is shown in Fig. 3.26. Isolated power for the neutral beam source will have the following parameters: arc supply, $150 \mathrm{~V}$ at $400 \mathrm{~A}$; filament supply, $12 \mathrm{~V}$ at $200 \mathrm{~A}$; magnet, $6 \mathrm{~V}$ at $75 \mathrm{~A}$. This power will be provided by an appropriate isolation transformer to the voltage isolated source deck. A one-line diagram of this system is shown in Fig. 3.26 .

Both the superconducting toroidal field coils and superconducting gyrotron magnet coils will be powered by special purpose high current dc power supplies. Each supply will have provisions for remote programming, remote sensing, automatic crossover, overcurrent cutoff, and reverse voltage protection. This type of supply is readily available from several manufacturers. Each of the 48 toroidal field coils will be powered by a separate power supply with a nominal rating of $10 \mathrm{~V}$ at $1500 \mathrm{~A}$. Each of the 12 gyrotron magnets will require four supplies with nominal ratings of $10 \mathrm{~V}$ al $600 \mathrm{~A}$. These supplies will be located in close proximity to the coils.

\subsubsection{Motor-generator sets}

Existing motor-generator sets in B1dg. 9201-2 will be used to power the AKE co1ls. Four 2.5-MW, $350 \mathrm{~V}$ at $7140 \mathrm{~A}$ and two 3.0-MW, $350 \mathrm{~V}$ at $8571 \mathrm{~A}$ generators will be available for use on EBT-II. Each of the 96 ARE coils has a requirement of $22 \mathrm{~V}$ at $6400 \mathrm{~A}$. Each generator will be used to drive 16 ARE coils connected in series.

\subsubsection{Grounding}

A building ground grid will be provided for structural steel and equipment grounding in the new power supply building. The ground grid and all equipment grounding will be in accordance with Sect. 5.12 of Union Carbide Corporation General Design Criteria Y-EF-538. 


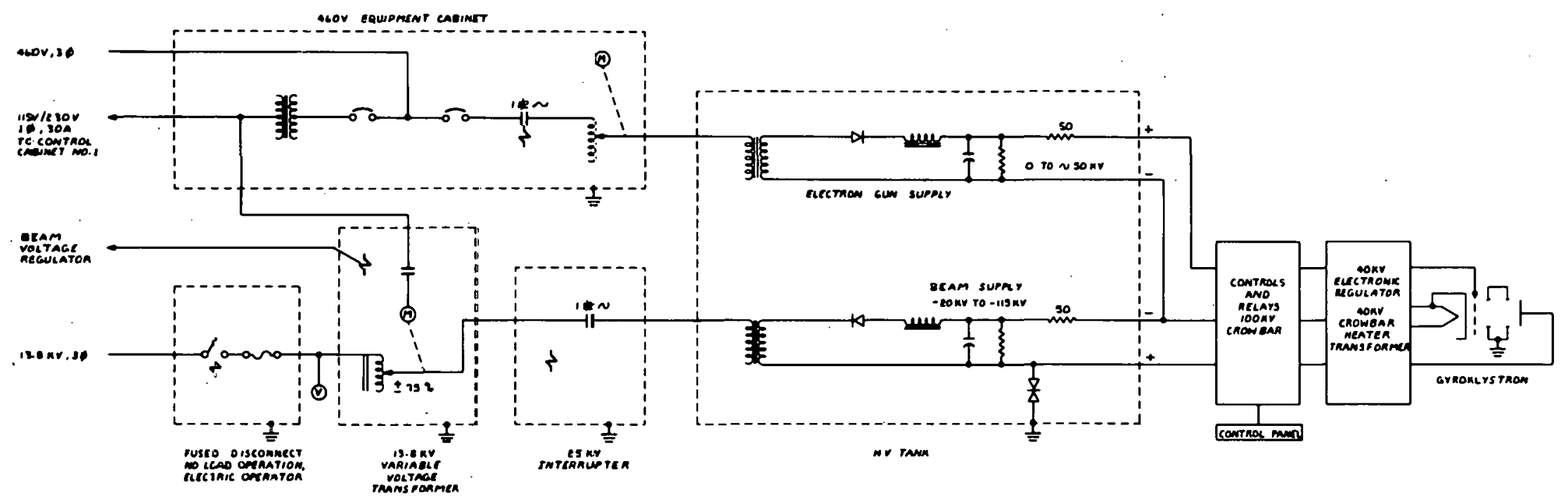

Fig. 3.24. Gyrotron power supply schematic. 

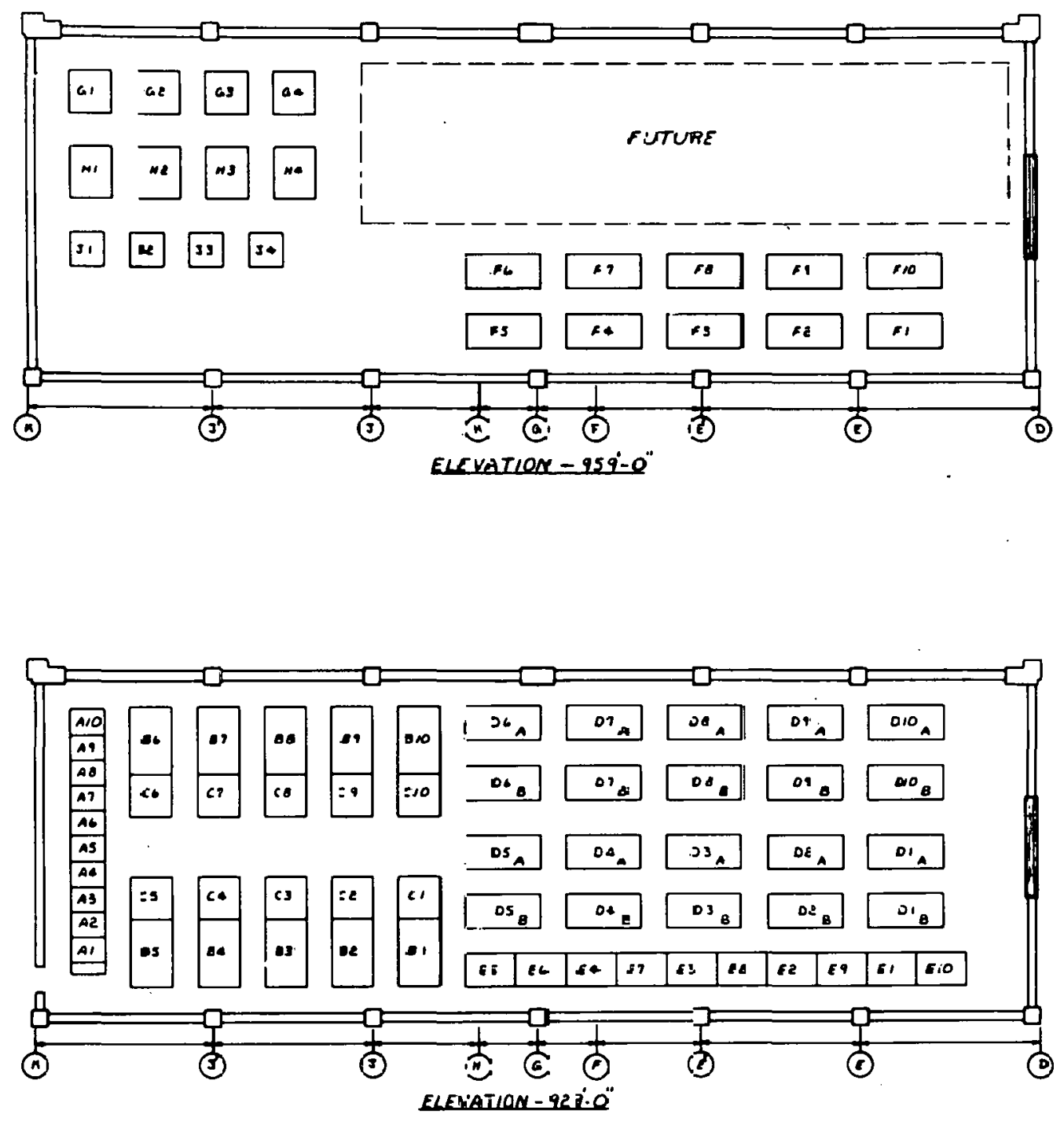

\section{EQUUDMEAT LEGEND}

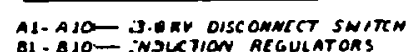

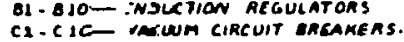

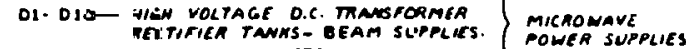

GI-EJE- : OWTAOL CABINETS

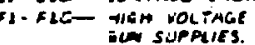

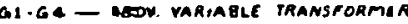

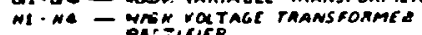

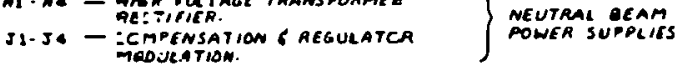

Fig. §.25. Fower supply building layout. 

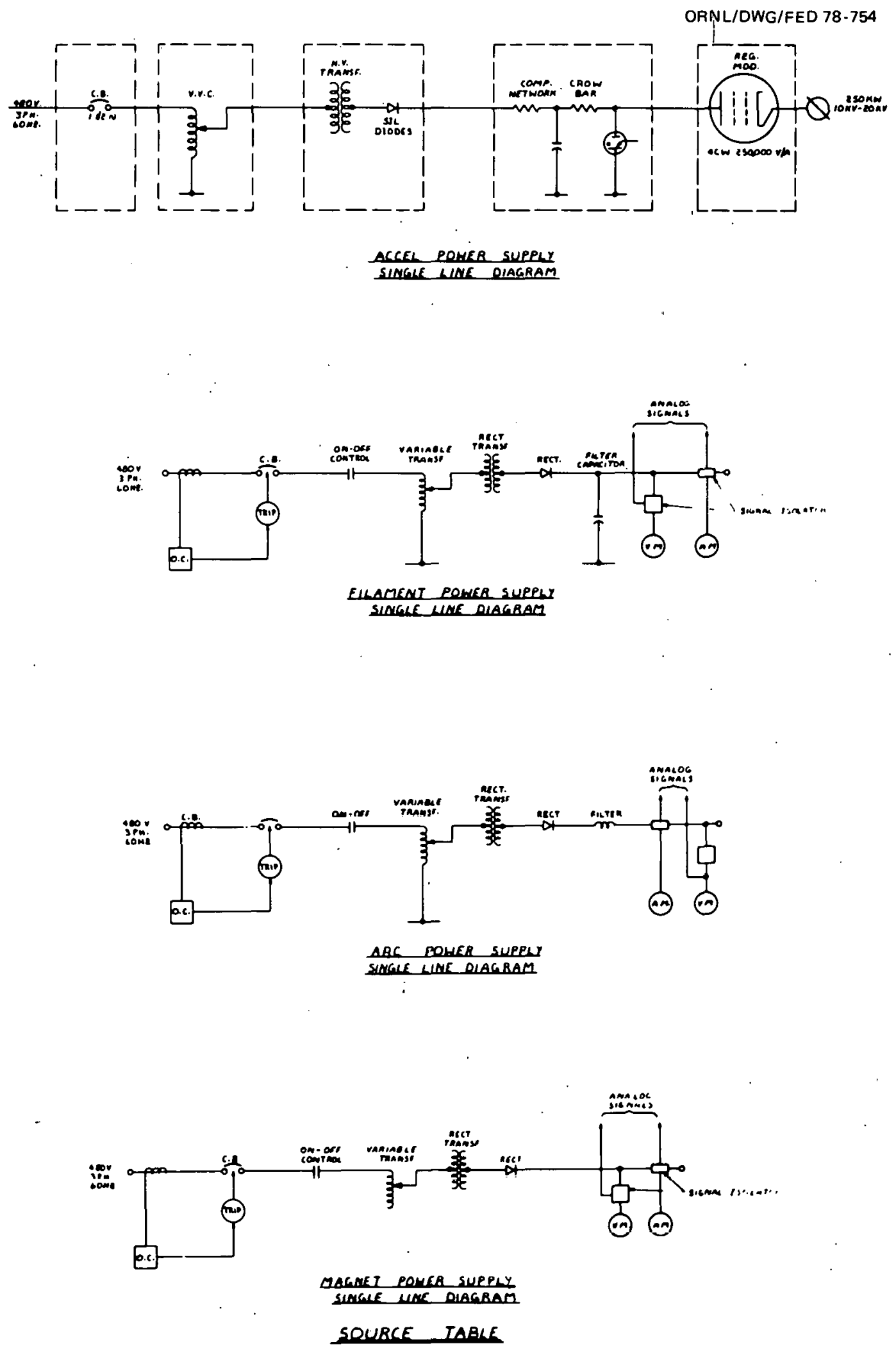

Fig. 3.26. Neutral bcam power supply schematic. 


\subsubsection{Lighting and receptacles}

High pressure sodium lighting will be used throughout the new power supply building. Illumination levels will be in accordance with current Department of Energy guidelines. Emergency exit lights will be installed where required. Duplex convenience receptacles will be installed throughout the new building. All lighting and receptacles will be in accordance with Sect. 5.13 of Y-EF-538.

\subsubsection{Fire protection}

The existing Bldg. 9201-2 fire alarm system will be extended to provide coverage for the power supply building. The fire alarm system will be in accordance with Sect. 2.5 of Y-EF-538.

\subsubsection{Communication and alarm systems}

The existing public address system and emergency evacuation system in Bldg. 9201-2 will be extended to provide coverage in the new power supply building.

\subsubsection{Wire, conduit, and raceway systems}

All wire, conduit, and raceway systems will be installed in accordance with Sect. 5.14 of Y-EF-538.

\subsubsection{Electrical equipment and circuit identification}

The circuit and equipment identification system will comply with Sect. 5.15 of Y-EF-538.

\subsubsection{Instrumentation and Control}

The control and monitoring of EBT-II will be accomplished by a combination of manual and digital devices with each subsystem capable of operation in both a manual and a computer assisted mode. The instrumentation requirements can be divided into the basic tasks of machine operations and plasma physics experiments. The emphasis here will be on instrumenting the machine for safe and reliable operation. It is considered desirable to form an ongoing record of both the essential machine parameters and data from the various experimental plasma diagnostics and provide different options for data reduction. This discussion of instrumentation of the plasma diagnostics is 1 imited to the features necessary to accomplish this aim.

Instrumentation for basic machine operation is divided into eight subsystems: auxiliary systems, neutral beam injectors. ARE coils, superconducting toroidal field coils, main control console, machine diagnostics, data acquisition, and radiation safety and interlocks. The instrumentation requirements are summarized in Table 3.7.

\subsubsection{Auxiliary systems}

The auxiliary systems consist of all utilities and services necessary for the successful and safe operation of EBT-II. As presently scoped, these systems consist of a vacuum system, liquid nitrogen supply, liquid helium supply, cooling water supply, and the necéssáry interlock c1rcultry to monitor and control the systems.

Vacuum systems. The EBT-II vacuum will be established by a system of roughing and turbomolecular pumps and 48 liquid helium cryocondensing pumps, one at each cavity (see Fig. 3.27). In addition, there will be four beam dumps, located diametrically opposite each of the proposed neutral beam injectors, 
Table 3.7. Proposed EBT-II instrumentation

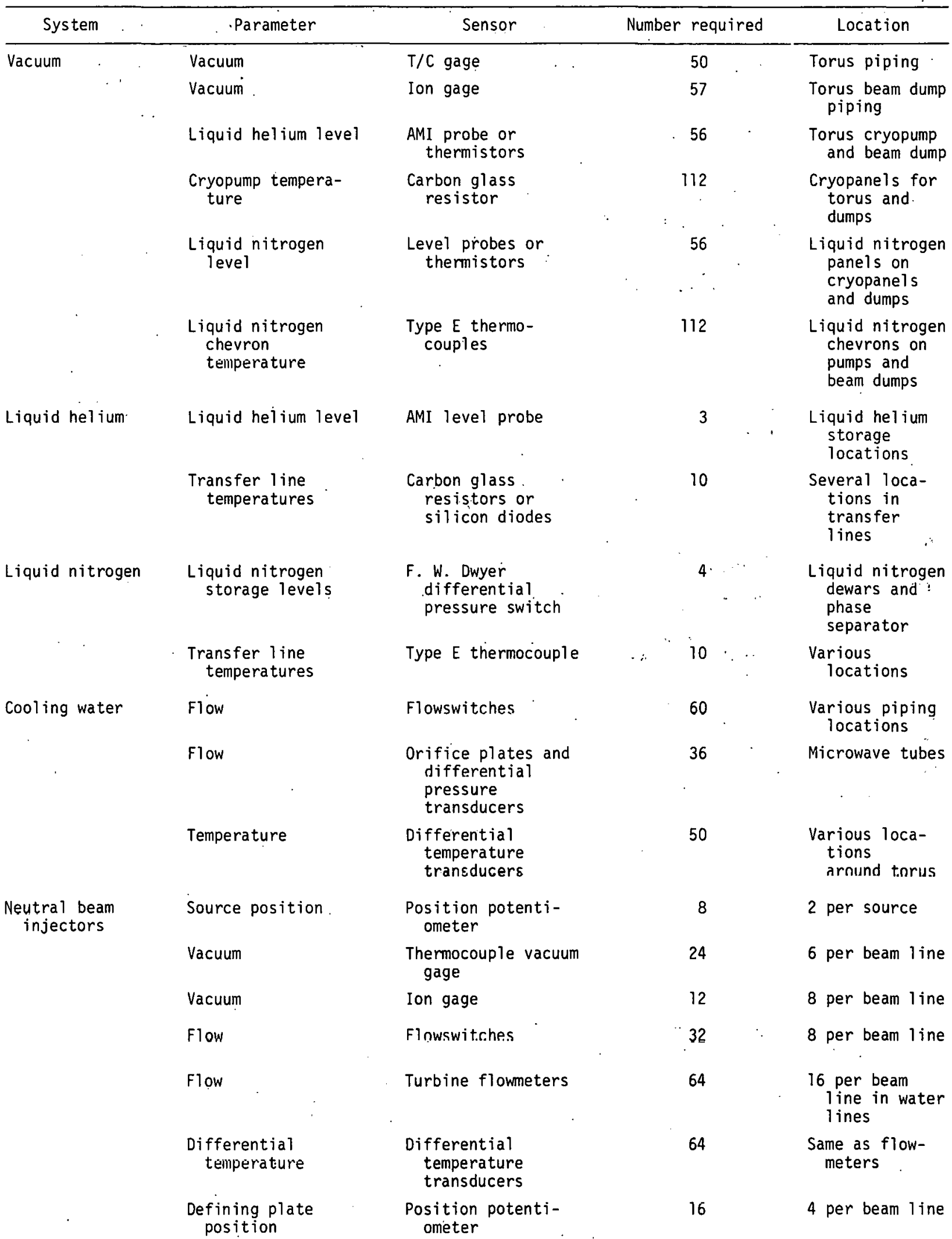


Table 3.7. (continued)

\begin{tabular}{|c|c|c|c|c|}
\hline System & Parameter & Sensor & Number required & Location \\
\hline \multirow[t]{5}{*}{$\begin{array}{l}\text { Neutral beam } \\
\text { injectors }\end{array}$} & Magnetic field & Hall probes & 8 & $\begin{array}{l}2 \text { per bending } \\
\text { magnet }\end{array}$ \\
\hline & $\begin{array}{l}\text { Liquid nitrogen } \\
\text { chevron tempera- } \\
\text { ture }\end{array}$ & $\begin{array}{l}\text { Type } E \text { thermo- } \\
\text { couples }\end{array}$ & 80 & $\begin{array}{l}\text { Liquid nitrogen } \\
\text { chevrons }\end{array}$ \\
\hline & $\begin{array}{l}\text { Liquid hel ium panel } \\
\text { temperature }\end{array}$ & $\begin{array}{l}\text { Carbon g.lass } \\
\text { resistors }\end{array}$ & 16 & $\begin{array}{l}\text { Liquid helium } \\
\text { panels }\end{array}$ \\
\hline & $\begin{array}{l}\text { Liquid nitrogen } \\
\text { level }\end{array}$ & $\begin{array}{l}\text { F. W. Dwyer } \\
\text { differential } \\
\text { pressure switch }\end{array}$ & .8 & $\begin{array}{l}\text { Liquid nitrogen } \\
\text { dewar on each } \\
\text { cryopump }\end{array}$ \\
\hline & Liquid helium level & AMI level probe & 8 & $\begin{array}{l}\text { Liquid hel ium } \\
\text { dewar on each } \\
\text { cryopump }\end{array}$ \\
\hline \multirow[t]{3}{*}{ ÁRE COIIS } & Current & $\begin{array}{l}\text { Current transducer/ } \\
\text { shunt }\end{array}$ & 96 & Current lead \\
\hline & Voltage & Potential taps & 96 & Magnet leads \\
\hline & Magnet field & Hall probes & 96 & $\begin{array}{l}\text { ARE coil } \\
\text { assembly }\end{array}$ \\
\hline \multirow[t]{7}{*}{$\begin{array}{l}\text { Superconducting } \\
\text { toroidal } \\
\text { field colls }\end{array}$} & Liquid helium level & $\begin{array}{l}\text { Discrete position } \\
\text { thermistor }\end{array}$ & 96 & 2 per magnet \\
\hline & $\begin{array}{l}\text { Vapor cooled lead } \\
\text { temperature }\end{array}$ & $\begin{array}{l}\text { Carbon glass } \\
\text { resistor }\end{array}$ & 96 & 1 per lead \\
\hline & $\begin{array}{l}\text { Vapor cooled lead } \\
\text { temperature }\end{array}$ & $\begin{array}{l}\text { Platinum resistance } \\
\text { thermometer }\end{array}$ & 96 & 1 per lead \\
\hline & $\begin{array}{l}\text { I iquid nitrngen } \\
\text { shield tempera- } \\
\text { ture }\end{array}$ & $\begin{array}{l}\text { Type E thermo- } \\
\text { couples }\end{array}$ & 86 & 2 per shield \\
\hline & $\begin{array}{l}\text { Liquid helium } \\
\text { temperatures }\end{array}$ & $\begin{array}{l}\text { Carbon glass } \\
\text { resistors }\end{array}$ & 96 & 2 per magnet \\
\hline & Magnet. quench & Rnġnwski coị & 48 & 1 per magnet \\
\hline & Magnet quench & Potential taps & 48 & 1 per magnet \\
\hline
\end{tabular}



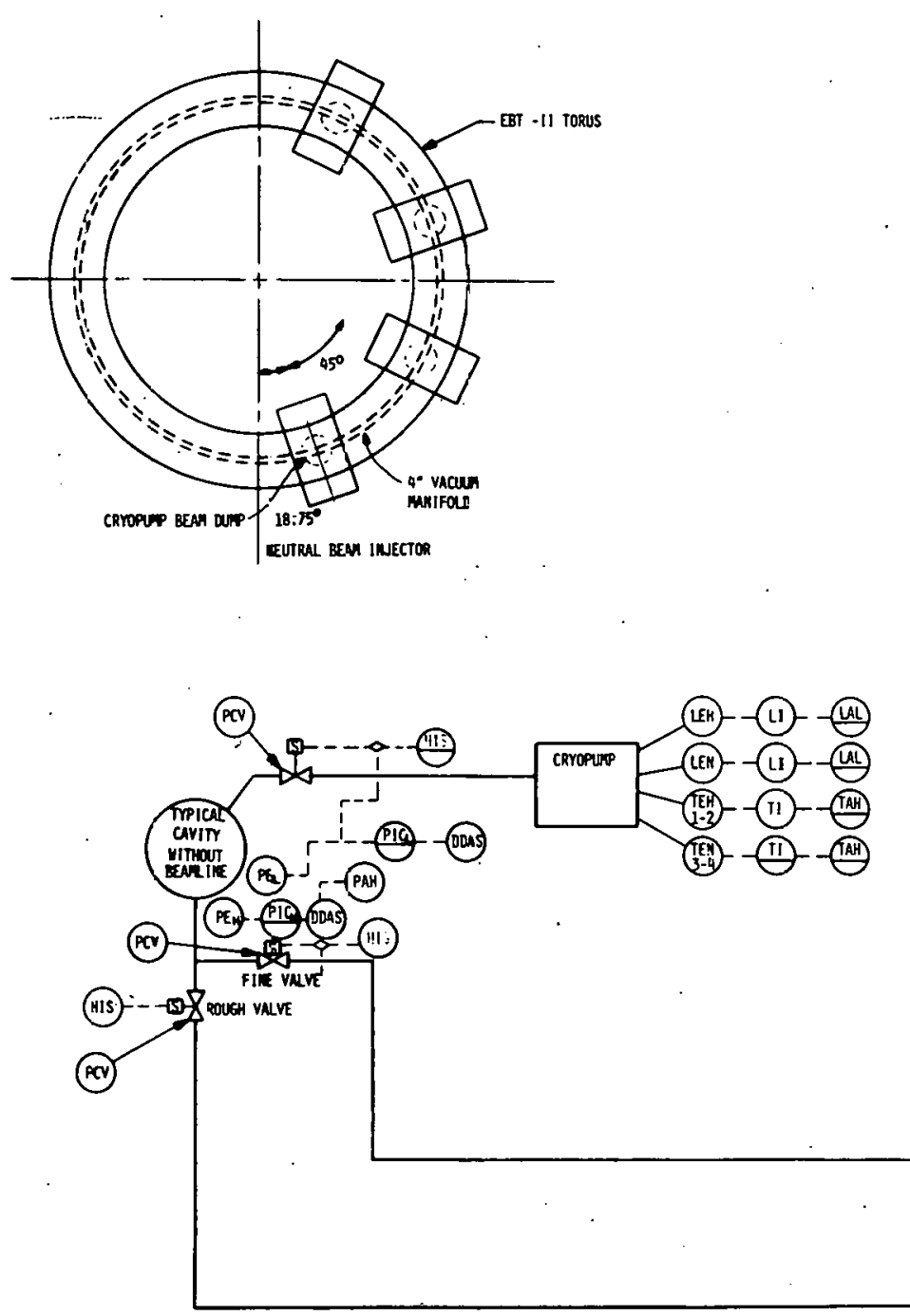

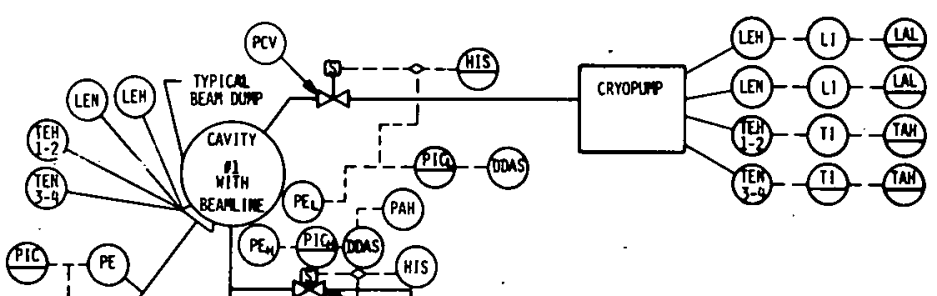

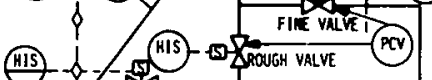

(IIS)

(ख)

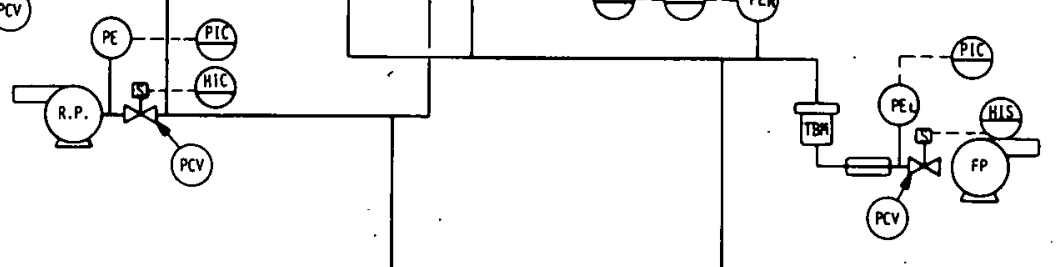

Fig. 3.27. Vacuum system instrumentation diagram. 
which will also be pumped by cryocondensing liquid helium pumps. The vacuum system instrumentation is shown in Fig. 3.27. It will consist of both standard vacuum instruments and the necessary instruments to monitor the cryopumps. The vacuum instruments will be a combination or thermocouple gages and ion gages to monitor both the rough and high torus vacuum. The cryopump wilt be monitored with liquid helium level probes, carbon glass resistors, and thermocouples. The liquid helium levels will be monitored by either a continuous reading superconducting probe or a set of discrete level thermistors. The sensors for the liquid helium will be those previously specified for the Large Coil Project. The vacuum system 'will be controiled by a programmable logic controller (PLC) and lhe uulpuL ur LIAis cuiltroller will act as a permissive interlock into the total operation plan for the EBT-II.

Liquid helium system (see Fig. 3.28). The liquid helium for EBT-II will be furnished by a closed loop refrigerator liquefier to be purchased on a performance specification. The refrigerator will be supplied to EBT-II with all instruments and controllers necessary for operation of the system, with the controllers ready for interfacing to a digital computer system designed by Union Carbide Corporation's Nuclear Division (UCC-ND). Also, UCC-ND will supply the liquid level monitoring for liquld licilum storage and temperature sensors for monitoring the transfer lines. The liquid helium storage levels will be monitored by AMI superconductiny pröbés or a differential pressurc lcvel measurement. Temperatures will be monitored by silicon diodes, carbon glass resistors, or a combination of both. In addition, a direct digital control system, based on a Digital Equipment Corporation (UEC) microcomputer and identical to the system proposed for the refrigerator liquefier on the Large Coil Project, will be used.

Liquid nitrogen (see Fig. 3.28). The liquid nitrugell for cooldown and cryopump chevrons will be supplied from an exterior trailer system. The instrumentation will consist of an automatic level controller on the phase separators based on a differential pressure measurement and on Type $E$ thermocouples placed on the transfer lines. The level controller will control cryogenic rated solenoid valves. The valves to be utilized will be the same as those used on the Medium Energy Test Facility and proposed for the Large Coil Project. The liquid nitrogen system will be monitored and controlled by a PLr.

Cooling water. All cooling water lines shall be monitored by a system of in-line flow switches and (where applicable) by differential temperature transducers, either the delca-T thermuplles ur a UCC-ND system presently under development. These signals will be monitored by a logic controllor and will provide permissives for machine operation.

Environmental considerations. The auxiliary systems are essential to the safe operation of EBT-II and in view of this, each critical variable or signal will be evaluated for signal isolation and magnetic field effects. The signals will then be conditioned to account for or correct the environmental effects. The techniques required for this either have been used ur dre beilly devieluped for other projes.t.s.

\subsubsection{Neutral beam injertors}

The neutral beam injectors will be instrumented as shown in Fig. 3.29. The instrullenlation will be based on designs proven in other neutral beam systems, except that the EBT-II sources are $\mathrm{cW}$ instead of pulsed. The source will be rated for $20 \mathrm{kV}$ at $12.5 \mathrm{~A}$. There will be an isolated source table and modulator capable of $\mathrm{Cw}$ operation. The source table will consist of a filament power supply, gas source valve power supply, arc power supply, dild auxiliary magnet power supply. The mudulator will be a high voltage switch tube capable of operating $\mathrm{cw}$ at the rated voltage and current, complete with all necessary controls. The modulator and source table will be monitored and controlled by a series of optical isolation links which allow monitoring and control from the control room. The transducers to be utilized will be determined during the detailed design. 
ORNL/DWG/FED 78-753

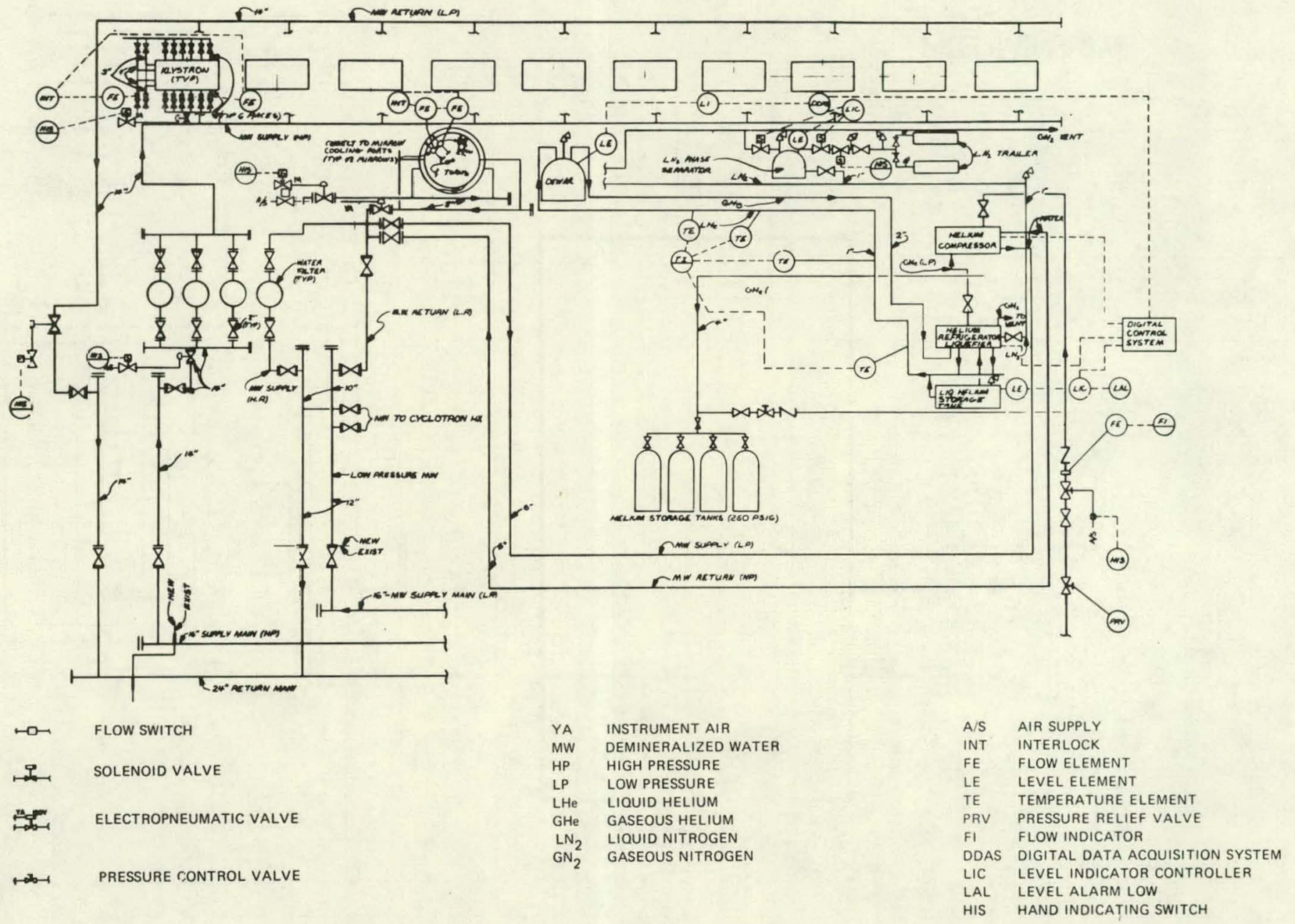

Fig. 3.28. Fluid flow instrumentation diagram. 


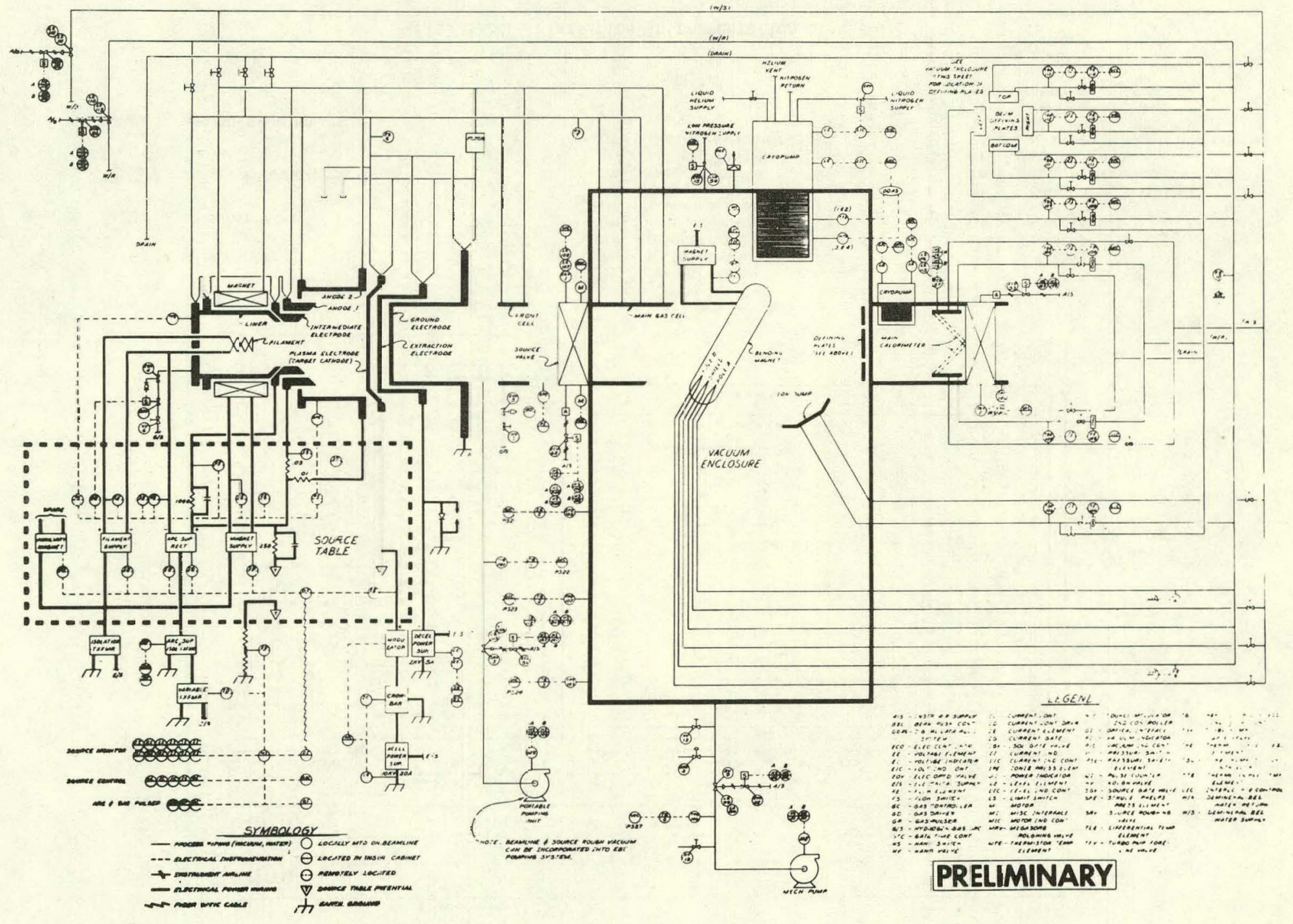

Fig. 3.29. Nestral beam line instrumentation diagram. 
The process instrumentation furnished on the beam lines will consist of a source gimbal controller, vacuum system controls, power monitoring instrumentation, cryogenic instrumentation for the cryocondensing pumps, aperture plate controls capable of remote operation, cooling water flow switches, and a magnetic field monitor.

The source will be gimballed by a dc servomotor operated from the control room. The source position will be indicated on a bidirectional digital panel meter and determined by an isolated potentiometer mechanically linked to the drive motor. The vacuum system will be monitored by a combination of thermocouple and ion gage controllers. In addition, a residual gas analyzer will be shared among the beam $I$ ines and EBT-II vacuum system. The output of the vacuum instrumentation will act as input to a logic interlock controller to guarantee safe operation of the beam lines and sequential operation with EBT-II. The beam line source power measurement will be made by a combination of differential temperature transducers and turbine flowmeters mounted in the cooling water lines and providing input to the digital data acquisition system for power calculations. This system has been utilized on previous beam lines. The beam line aperture plates will be controlled with the same system as the source gimbal. The cryopumps will be monitored by a combination of thermocouples, cryogenic thermistors, and liquid helium and liquid nitrogen level probes.

The beam lines will be supplied as individual modular units; each line will stand alone. They will be individually controlled by PLC's which act as input to a central PLC for all four beam 1 ines and provide the permissive interlocks to the EBT-II control system.

\subsubsection{Aspect ratio enhancement coils.}

The aspect ratio enhancement (ARE) coils (see Fig. 3.30) will be normal coils with a nominal current of $6400 \mathrm{~A}$. The instrumentation to these coils will consist of a Hall probe magnetic field monitor, a coil voltage measurement, and a coil current shunt. The signals will be conditioned with isolated amplifiers and stored on a digital data system. In addition to the instrumentation, a protection circuit will be provided to safely discharge the coils in case of power failure or accident. The coils will be controlled and all alarm conditions monitored by a logic controller.

\subsubsection{Superconducting toroidal field coils}

There is a total of 48 superconducting toroidal field or mirror coils (see Fig. 3.31). Each coil will have an individual power supply with a remote programming capability to allow the digital data system to be used to controi the fields. The coils shall also act as input to the logic rontrol system to provide safe and efficient control of the overall EBT-II experiment. Each coil will have the following instrumentation (based on techniques developed for the Large coil Program). The liquid helium level will be monitored by thermistors spaced up the coil sides to provide discrete measurement points. The vapor cooled leads will be monitored with a combination of carbon glass resistors and platinum resistance thermometers. The liquid nitrogen chevrons will be monitored with thermocouples and liquid helium temperatures with carbon glass resistors. In addition, voltage taps and Rogowski coils will be used to quench the coil. Each of these items will use the signal conditioning circuitry designed for the Large Coil Program.

\subsubsection{Master control console}

The main control console will contain indicators and analog inputs which present the status of the variọus subsystems. A set of status lights will indicate that each step to be performed has the necessary interlocks, as indicated by the PLC's. A set of machine timers will be provided to allow the various experiments to obtain correlated data when necessary. 


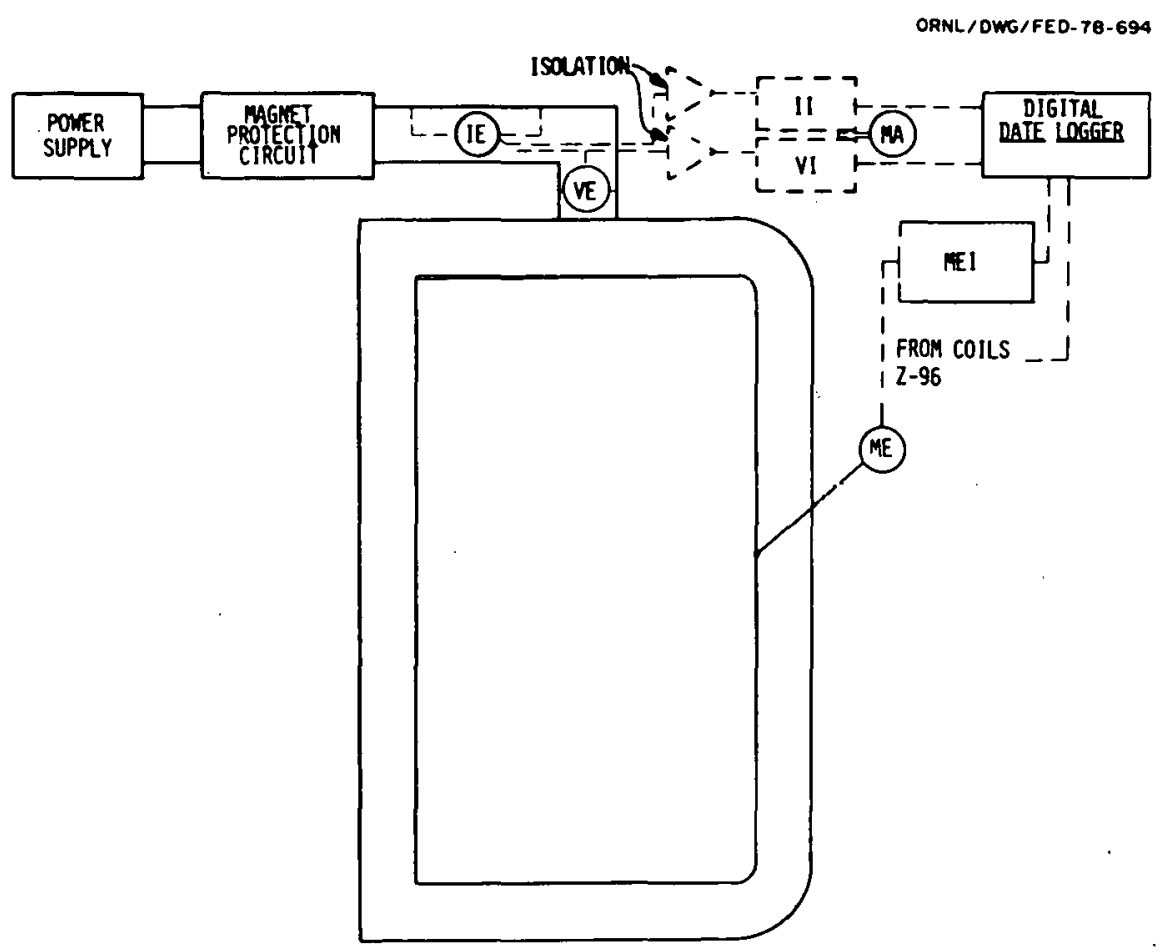

Fig. 3.30. ARE coil instrumentation schematic.

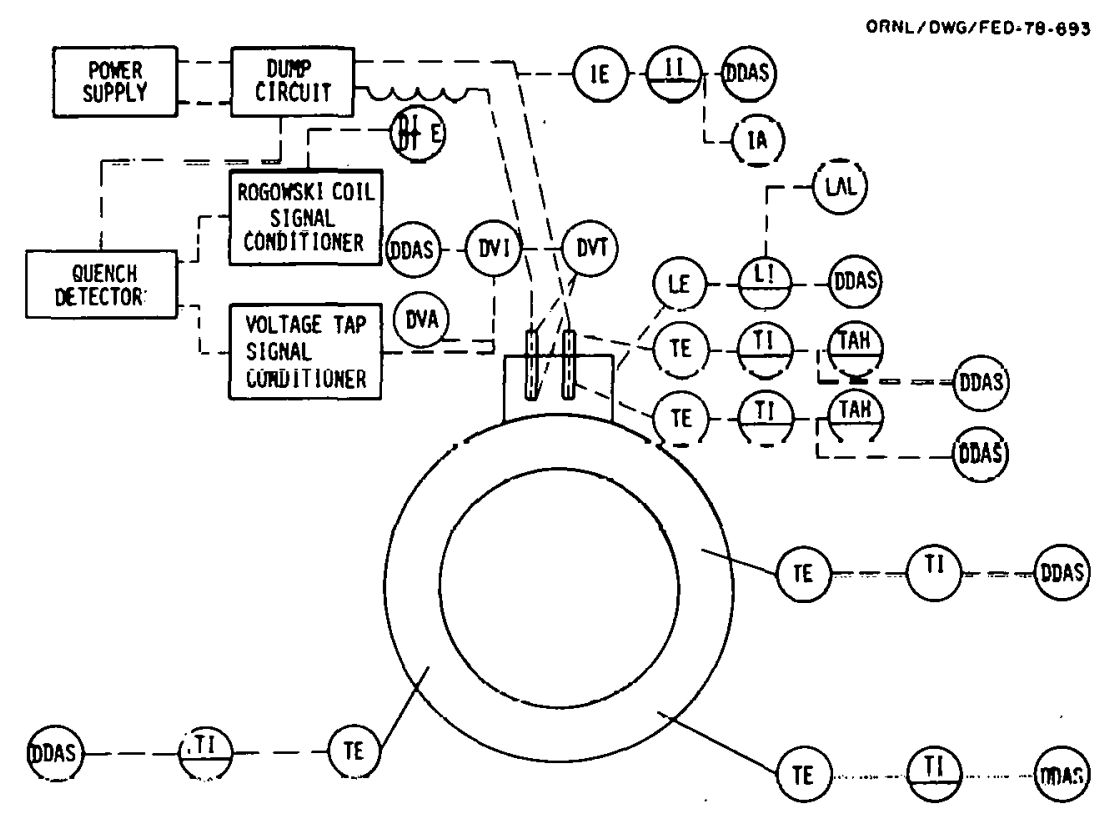

Fig. 3.31. Mirror coil instrumentation schematic. 
There will be a cathode ray tube (CRT) display upon which data can be examined from all on-1ine experiments and machine operations. A keyboard interface to the main data acquisition and control system will allow the operator to examine any data necessary for machine operation. In addition, a set of digital panel meters with front switches will permit viewing of selected parameters.

\subsubsection{Machine diagnostics}

The machine diagnostics consist of those transducers necessary to operate the machine in a safe and reliable manner over its full dynamic range.

Included in this category are the diamagnetic loops, toroidal current sensors, selected thermocouples and strain gages, a 1-mm interferometer for density measurements, and a measurement of the vacuum in each cavity. Analog inputs from these devices will be available at the main control console and will act as input to the main data acquisition system. Controllers for changing the gas density and trim magnet settings will be present at the main control console as mentioned earlier. Provisions will be made on the machlne and in the data acquisition system for additional diagnostics as specified by the experimenters.

\subsubsection{Data acquisition and interlocks}

EBT-II will be divided into a series of modules for purposes of data acquisition and interlocking. The interlocking function and the data acquisition functions will be separate, as will the low speed or slowly varying and the high speed or rapidly varying signals. The EBT-II interlock functions will consist of system inputs from the neutral beam system, the auxiliary systems, the microwave system, and the superconducting magnet systems to provide for the safe, sequential, and orderly operation of EBT-II. The interlock functions will be those compatible with relay logic, limit switches, pushbuttons, flow switches, and other process equipment. Because traditional relay logic is inflexible, hard to modify, and, with the number of interrelated subsystems, not practical for a system as complex as EBT-II, the interlocks will be handled by a system of solid-state PLC's. The system will have complete optical isolation of inputs and outputs and can be easily modified as experiments change. Each subsystem of ERT-II will be broken down into as small a module as is practical for control purposes; each of the subsystems is discussed.

The initial number of neutral beams is four with the possibility of an increase to eight, depending on the experimental requirements. The neutral beams will be furnished as modules including power supplies. For this reasuil, each ncutral beam system will be interlocked through a PLC. The controller will accept inputs from the process variables in the form of contact closures, flow switches, limit switches, and vacuum gage outputs and will provide the necessary front panel indications to the operator to guarantee the startup and operation of the beam line. The output of the PLC is compatible with digital computers; it communicates with the computer through a standard data link allowing either local or remote startup of one through four beam lines.

The interlock functions for the micrnwave systems, superconducting magnet power supplies, and auxiliary systems will also be handled by PLC's. The description is the same as for the neutral beam injectors and in each installe, the control scheme will he made as modular as possible to prevent any component failures from unnecessarily preventing machine operation.

The EBT-II data acquisition system must be evaluated in terms of scanning speeds required. There is a large number of relatively constant output signals that have no bearing on the experimental results and are provided to verify correct machine operation. These comprise all the signals relating to the operation of the cryogenic systems and most of the facility variables. It is proposed that these 
signals be input through a slow speed scanner into a DEC 16-bit microcomputer system. This system shall act as a stand-alone unit dedicated to verifying that the facility and cryogenic systems are functioning and to alerting the operator through a series of multilevel alarm points of any system abnormalities. In addition, the microcomputer shall communicate with the main facility through a standard RS-232C communication 1 ink.

In previous discussions with refrigerator liquefier vendors, it was decided that large-scale refrigerator liquefiers require some method of centralized automatic control and data acquisition. This will be accomplished by specifying that all refrigerator controls be compatible with a digital computer (basically the system proposed for the Large Coil Programs), based on a DEC microcomputer that acts as a stand-alone system and communicates with the central EBT-II data system through an RS-232C interface.

The central EBT-II digital computer will be a medium-sized lo-bit minicumpuler with disc storage and a 9-track magnetic tape unit. The real-time operating system shall have multitask, multiterminal capabilities with an extended FORTRAN IV compiler and floating point hardware. In general, nn critical real-time monitoring or control functions will be assigned to this system; its primary purpose is to provide an output data set containing the machine operating conditions and plasma diagnostic data. In addition, the system will provide CRT displays of these data with intermediate analysis and/or data reduction as well as hard copy listings. The system will be able to send controlling outputs, which are not part of a critical control function, to machine subsystems as desired. A block diagram of this system is shown in Fig. 3.32, and the overall data system is shown in Fig. 3.33. Interface to the plasma physics experiments will be CAMAC based, utilizing either parallel or serial data transfer. The structure of CAMAC is such that both the experiment and the data acquisition systell will receive minimal impact when changes are required, which permits more capability for data acquisition under a variety of conditions.

\section{$3.1 . \%$ Radiation and personnel safety}

A system of instruments and interlocks will be provided to protect personnel against radiatinn and inadvertent entry to any hazardous area during an experiment. Kirk key-type interlocks will regulale operation of the high voltage, use of laser diagnostics, and energizing of the ARE or toroidal field colls. Unless this system is actịve, the nespssary permissives for the PLC'3 eannot be mel and llie system cannot be operated. In addition, each source gage on the beam lines will have a key operated interlock. The radiation monitors will use a scintillation-type detector and a ratemeter with a front adjustable alarm feature. If neutrons are generated by D-D or D T reactions, $A$ sel ur BF dutectors and associated counters will be provided.

\subsubsection{Structural Considerations}

\subsubsection{Torus enclosure}

The EBT-II experiment enclosure wili be located on the second floor of Bldg. 9201-2, as shown in Fig. 3.2 (between columns 4,7, $H$, and $K$ ). The enclosure will consist of a 53-ft-wide octagonal shield ring of 2-ft-thick by 21-ft-high concrete walls. The roof over the enclosure will be shieided with a 4-in.-thick layer of lead. An operating platform will be provided with a 100-lb/ft ${ }^{2}$ floor 1 ive load capacity approximately $9 \mathrm{ft}$ above the second floor level, and a 2-ton hoist will be provided to.assist in moving equipment either to or from the operating platform.

The experiment coil supports will consist of a series of 10- x 48-in., 10-ft-high concrete columns, each capped with a 48-in.-wide by 15-in.-thick continuously reinforced concrete ring. 
ORNL/DWG/FED 78.747

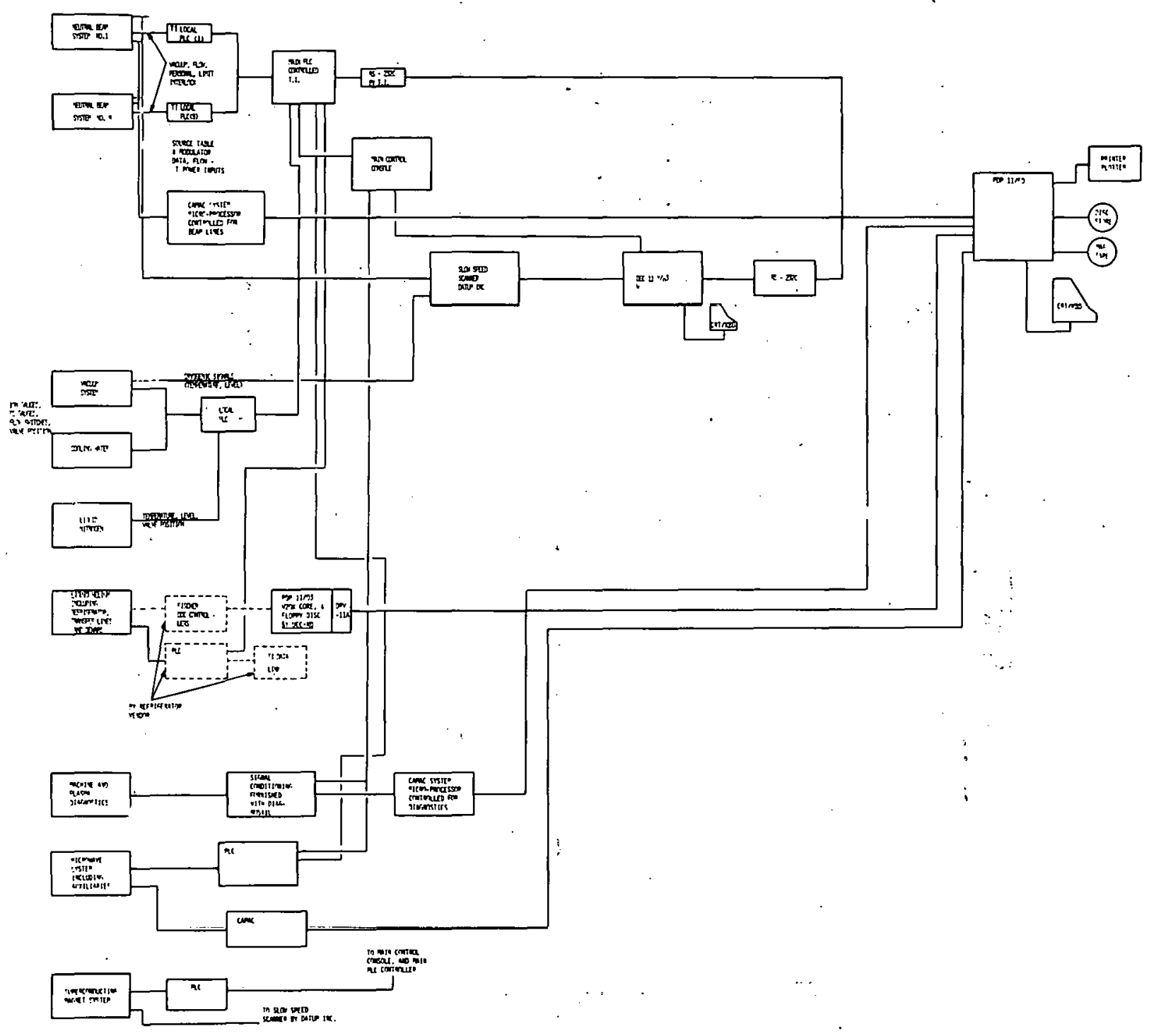

Fig. 3.32. Data acquisition system schematic. 
ORNL/DWG/FED $78 \cdot 750$

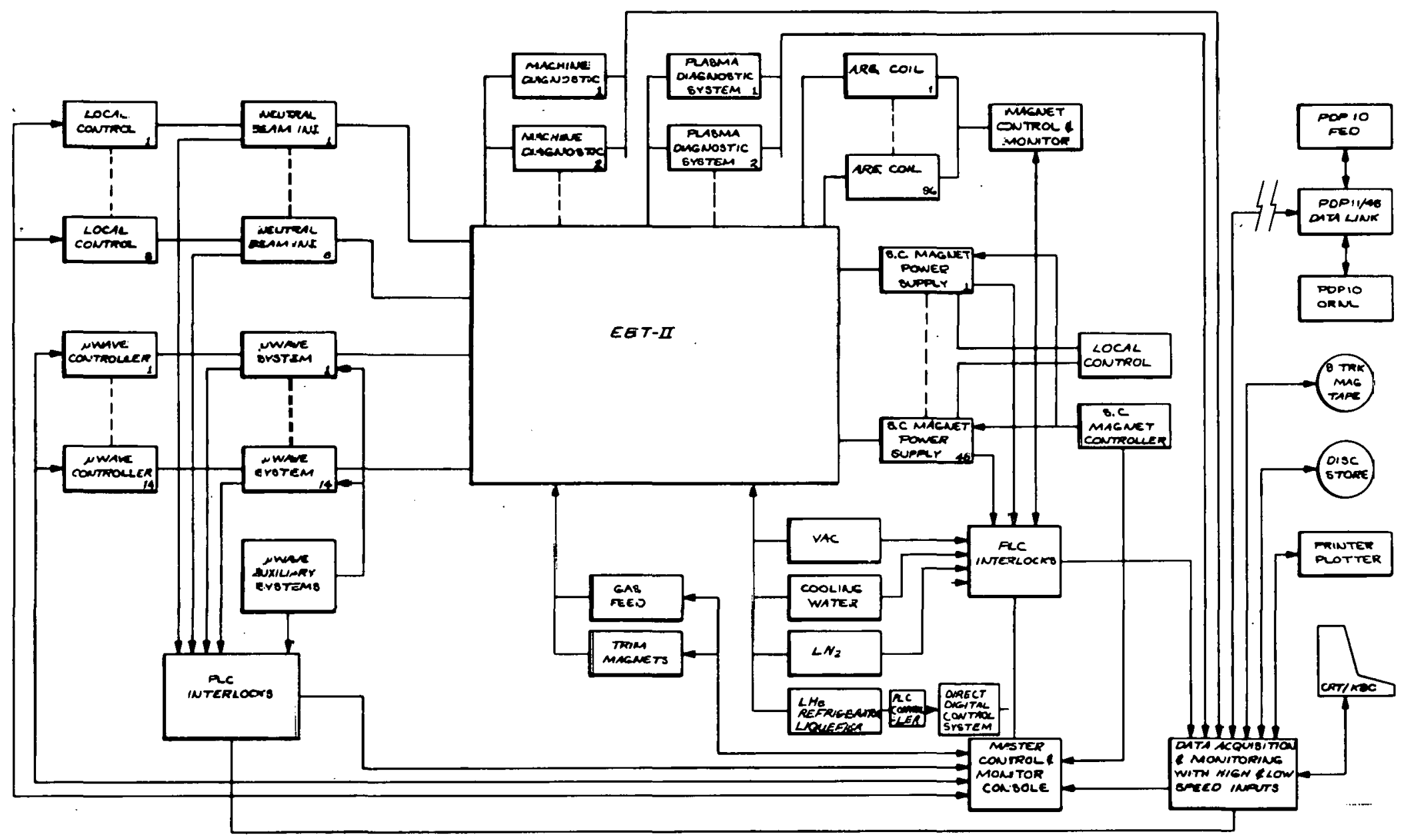

Fig. ¿.33. Data system schema-ic. 
Additional structural support for the experiment enclosure is necessary and will consist of steel framing placed under the concrete floor slab and 13 new steel columns extending down to the building foundation level, where they will bear on individual spread footings. The operational live load on the second floor is $300 \mathrm{lb} / \mathrm{ft}^{2}$.

\subsubsection{Mechanical equipment enclosure}

The helium refrigeration system enclosure (see Fig. 3.2) will be located on the low control bay roof, as shown in Fig. 3.2 (between columns $4,7, K$, and $M$ ). It will consist of a 25-ft-high steel framed enclosure with corrugated metal roofing and siding.

The new equipment inside the enclosure will be supported by a special structural framing system which will tie in directly to the building columns. In addition, there will be an acoustical isolation system consisting of a 2-in.-thick layer of styrofoam board topped with a 4-in.-thick reinforced concrete wear surface over the entire floor area inside the enclosure.

Access will be through one 3-ft-wide by 7-ft-high personnel door and one 6-ft-wide by 8-ft-high set of double swinging doors. Concrete ramps shall be provided at each door.

The helium refrigeration compressor shall be located on a reinforced concrete pad on the ground at the northwest corner of $81 \mathrm{dg}$. 9201-2, alongside the liquid nitrogen trailers. The compressor will be protected with a steel chain link fence and five standard bumper guard posts.

A 2-ft-thick by 15-ft-wide by 90-ft-long reinforced concrete support slab will be provided for the high pressure helium storage tanks approximately $70 \mathrm{ft}$ southwest of Bldg. 9201-2 (see Fig. 3.34). In addition, three 24-ft-high steel tube pipe supports shall be provided for the helium supply line from the tanks to B1dg. $9201-2$.

\subsubsection{Control room}

The control room for EBT-II will be located on the second floor of Bldg. 9201-2, adjacent to the experiment enclosure, as shown in Fig. 3.2 (between columns 2, 4, $H$, and $K$ ). The facility will consist of a 45-ft-ling by 24-ft-wide, metal paneled, structural steel frame enclosure with access through an 8-fl-high by $6-f t-w i d c$ set of double swinging hollow metal doors in both the north and south end walls. The room will have a suspended acoustical ceiling.

\subsubsection{Power supply enclosure}

The 10-MW power supply vault addition to the west end of Bldg. 9201-2 will be $120 \mathrm{ft}$ long by $42 \mathrm{ft}$ wide, with two 25-ft-high stories. The structure will be a reinforced concrete frame with monolithic one-way slab and beam construction. Exterior walls shall be 1 -ft-thick reinforced concrete block, providing 4-hr fire protection.

The allowable floor live loads shall be $300 \mathrm{lb} / \mathrm{ft}^{2}$ at each level, with the footings and columns sized to accommodate a future third level at the same capacity.

Access shall be provided by three 3-ft-wide by 7-ft-high personnel doors and one 12-ft-high by 10-ft-wide overhead rollup duor at the ground floor (1-in. level) with two sets of exterior metal stairs (one at each end of the building) and two personnel doors at the second level.

A 15-ton capacity bridge crane and motor operated hoist will be provided on each floor. Each one will be operational over the entire building. A $12 \times 15-\mathrm{ft}$ opening will be provided through the second floor at the north end of the building. 
ORNL/DWG/FED 78-758

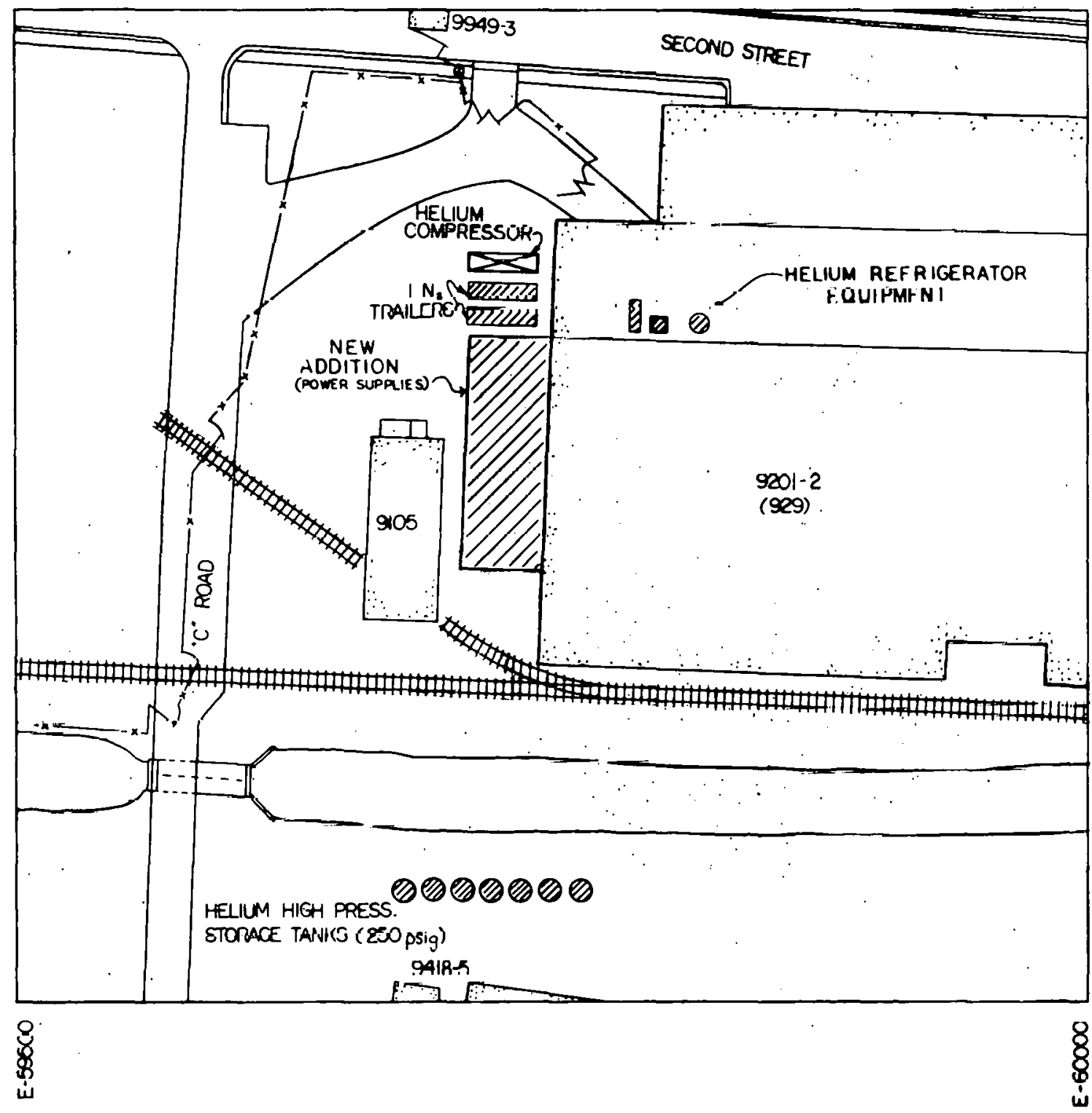

$N-29500$

$N-29000$

Fig. 3.34. Helium refrigerator equipment location. 
Prior to installation of the vault, the existing stairwell on the west wall of Bldg. 9201-2 and the remains of Bldg. 9732-2 shall be site cleared. The existing doors in the west wall of $81 \mathrm{dg}$. 9201-2 will be maintained, with the exception of the stairwell exit at the second floor level. This must be raised $5 \mathrm{ft}$ and a steel ramp must be provided to compensate for the difference in elevation between the two floors. The existing windows on the west end of the building, between columns $D$ and $K$, will be removed and the openings filled with brick.

\subsubsection{Mechanical Systems}

\subsubsection{Cryogenic systems}

A helium refrigerator/liquefier system and a liquid nitrogen system will be purchased and installed to supply the cryogenics required by the superconducting coils, neutral beam dumps and cryopanels, torus cryopumps, and focusing solenoids.

The helium refrigerator/liquefier will have a nominal capacity of 500 liters/hr of liquid helium at $4.2 \mathrm{~K}$ plus $1750 \mathrm{~W}$ of refrigeration at $4.2 \mathrm{~K}$. Major components include the cold box, compressors, a 4000-gai liquid helium storage tank, seven 25,000-gal high pressure helium gas storage tanks, and vacuum jacketed helium transfer pipe with and without liquid nitrogen tracing. The cold box and 1 iquid helium storage tank will be located on the low bay roof (see Fig. 3.35) to minimize the length of cryogenic helium transfer piping. The helium compressors will be located just west of B1dg. 9201-2. The high pressure (250-psig) helium gas storage tanks will be located outside the building, and gas will be transferred to and from these tanks via a 4-in. pipe. The system will be connected to the plant helium gas system to provide initial helium charging and makeup gas.

Liquid nitrogen will be used for initial coil cooling (to 77K), coil thermal shielding, torus cryopumps, neutral beam dumps, and cryopanels and for intermediate cooling within the helium refrigerator/liquefier. The system includes two 4000-gal liquid nitrogen trailers, a phase separator, and vacuum jacketed transfer piping. The trailers will be located just west of Bldg. 9201-2. The cryogenic helium and nitrogen systems are shown schematically in Fig. 3.36.

\subsubsection{Utility piping}

Piping utilities for EBT-II will include high and low pressure demineralized water, instrument air. and low pressure steam and condensate (see Fig. 3.36).

The 12 microwave generators will require approximately $550 \mathrm{gpm}$ each of high pressure demineralized water for a total requirement of $6600 \mathrm{gpm}$. A total of $200 \mathrm{gpm}$ of high pressure demineralized water will be supplied to the neutral beam injectors, and $75 \mathrm{gpm}$ will be supplied to the microwave reflectors. New spool pieces will be installed on the ends of the main building headers to facilitate new 16-in. supply and return branch lines to the EBT-II site. Four 1650-gpm capacity water filters will be purchased and installed in parallel upstream of the microwave generators.

Approximately 70 linear feet of 8-in.-diam low pressure demineralized water supply and return pipe now used for cyclotron heat exchanger coolant will be replaced with 12-in. pipe to accommodate both EBT-II and cyclotron requirements. At the end of the replacement pipe run, tees will be installed to branch to the cyclotron heat exchangers, and 12-in. pipe supply and return headers will continue to the EBT-II site. Three 1650-ypm filters will precede supply branches to the ARE coils (3110 gpm), the torus coils (340 gpm), and the hel ium compressor (1500 gpm).

Steam piping will be installed from the reducing station at the northwest corner of B1dg. 9201-2 to the west building addition. The pipe will provide $60-16 / h r$ stcam at 25 psig for heating the new building addition for the power supply enclosure. 


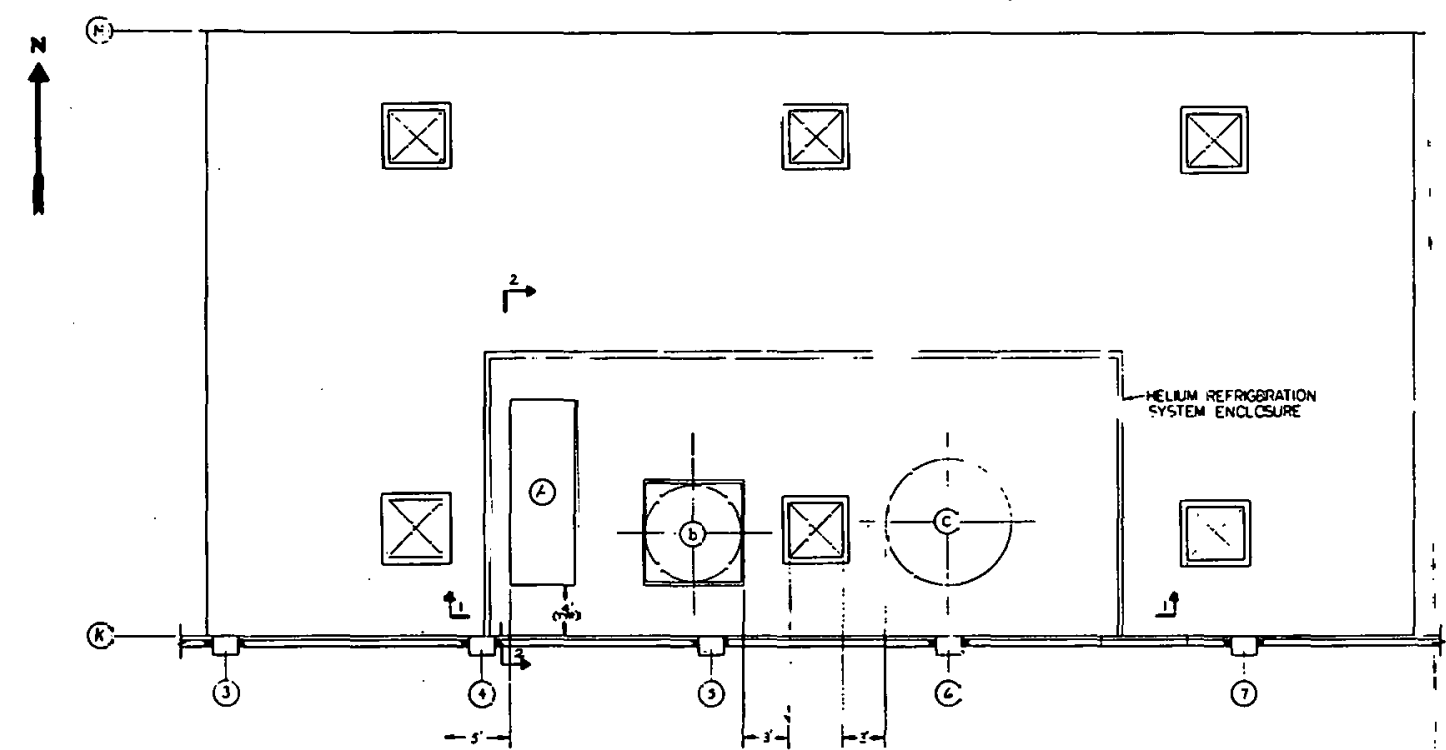

FARTILL ROOF PLAN
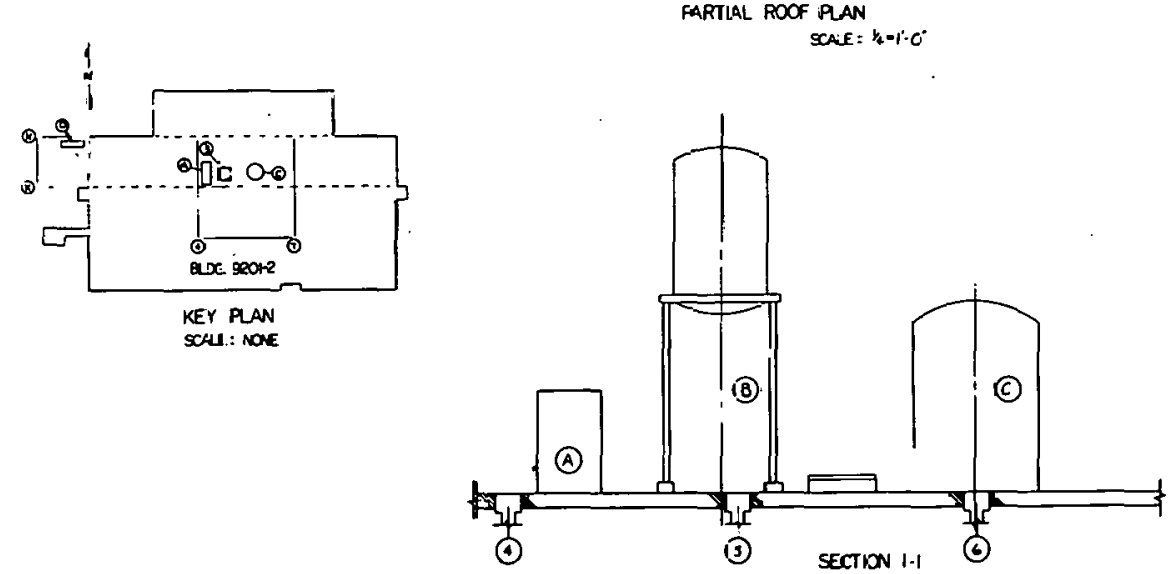

EQLIPRENT SOEORE

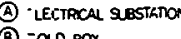

(D) 201100 Hessor

Fig. 3.35. Cutside equipment location.

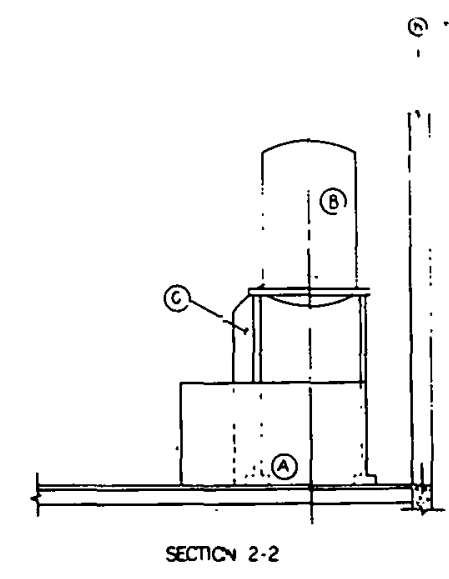




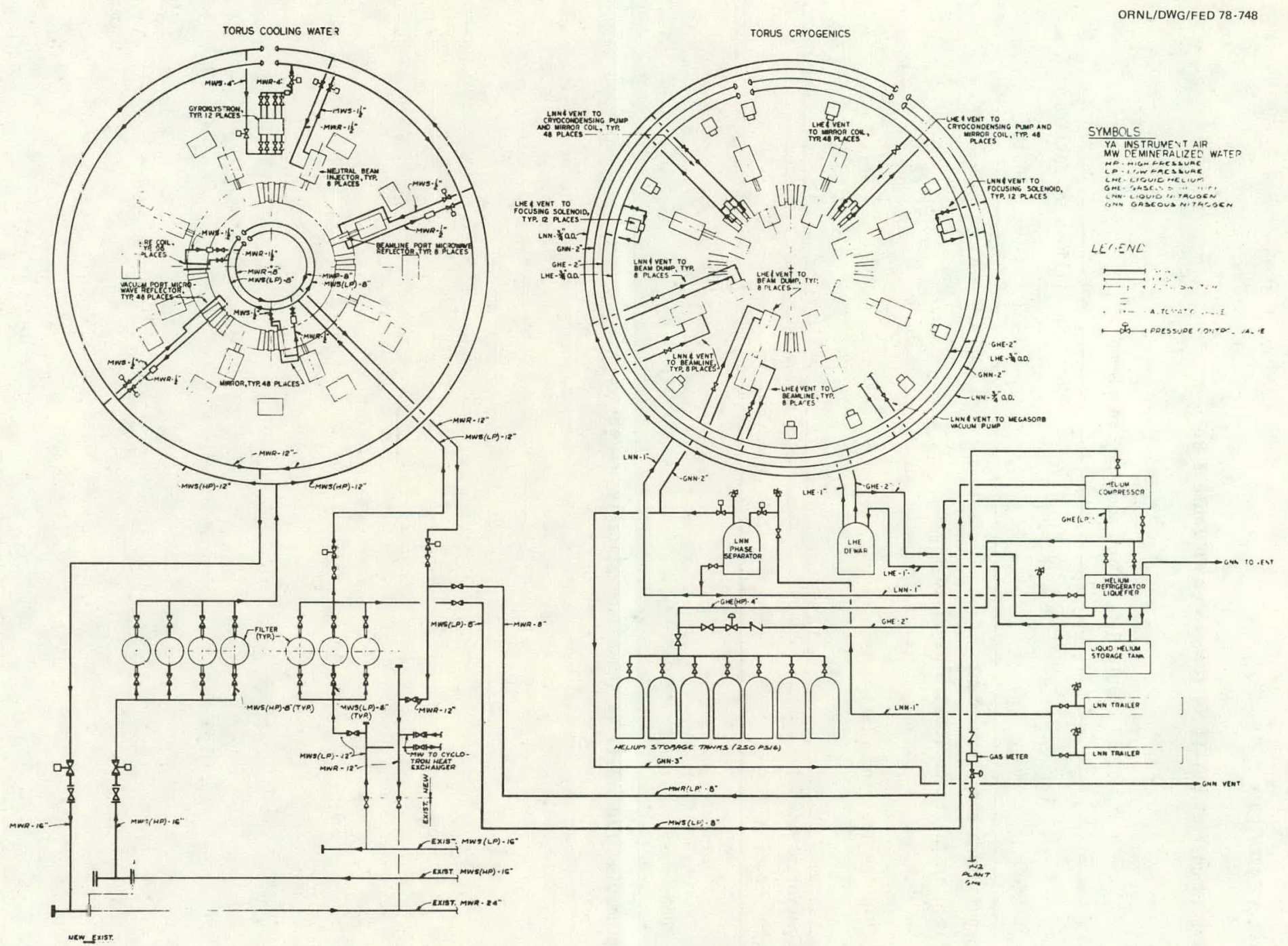

Fig. 3.36. Fluids flow system schematic. 
Instrument air piping from the $\mathrm{Y}-12 \mathrm{Plant}$ system will be used as required for valve operators and instrumentation.

\subsection{COST SUMMARY OVERVIEW}

The estimated cost of EBT-II is summarized in Table 3.8 .

Table 3.8. Cost summary overview

\begin{tabular}{|c|c|c|}
\hline & \multicolumn{2}{|c|}{ Cost (in millions of dollars) } \\
\hline & 1978 & 1981 \\
\hline Manufacture and procurement & 23.8 & 30.7 \\
\hline \multirow[t]{2}{*}{ Field installation (cost plus fixed fee) } & 10.7 & 14.0 \\
\hline & 34.5 & 44.7 \\
\hline \multirow[t]{2}{*}{ UCC-ND Engineering (25\%) } & & 11.2 \\
\hline & & 55.9 \\
\hline Contingency $(25 \%)$ & & 14.1 \\
\hline Total & & 70.0 \\
\hline
\end{tabular}

\subsection{PROGRAM PLAN}

The program plan for EBT-II is shown schematically in Table 3.9. 
- Table 3.9. Program plan

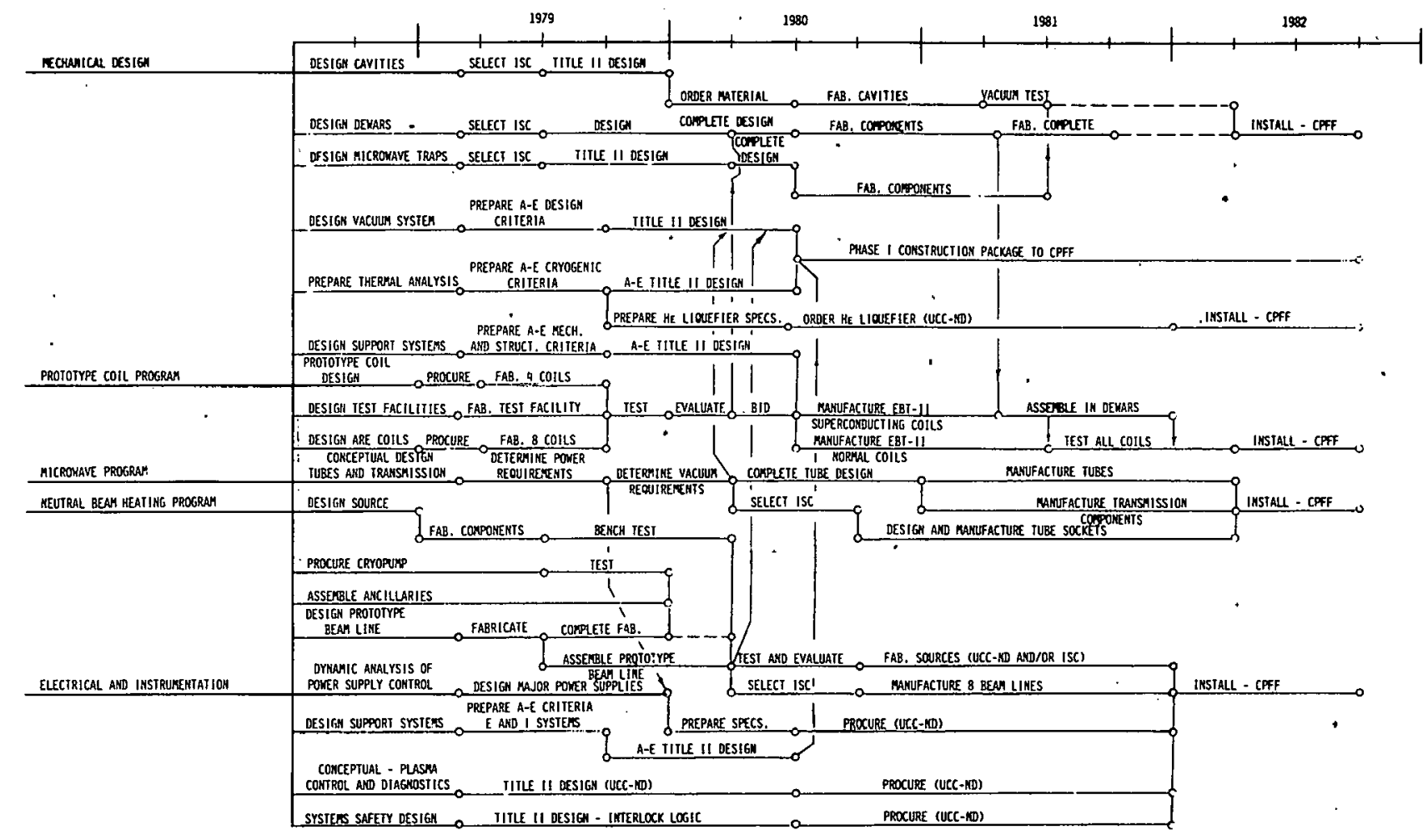


THIS PAGE

WAS INTENTIONALLY

LEFT BLANK 


\section{SAFETY MEASURES}

\subsection{SAFETY ACTIVITIES}

\subsubsection{Division and Plant Safety Activities}

The EBT-II facility will be located in Bldg. 9201-2 at the $\gamma-12$ Plant and will be operated by the Fusion Energy Division of Oak Ridge National Laboratory (ORNL). It will thus be subject to the safety programs of both $Y-12$ and ORNL.

The $Y-12$ safety policy is defined as follows:

"It is the policy of the $\gamma-12$ Plant to establish an effective accident prevention program and to maintain the necessary staff, service, and advisory groups to assist line supervision who are responsible for the safety of employees and equipment. Whenever our safety objective conflicts with our other objectives, safety shall be our first consideration."

This policy, is implemented by the Plant Superintendent, by a Central Safety Committee consisting of division and shift superintendents, by three other safety committees at lower levels, by a Safety Department, and by a Radiation Safety Department.

Within the Fusion Energy Division, a permanent Division Safety Officer provides liaison to and ensures compliance with the $\gamma-12$ Safety Program and oversees the Division safety activities. The Fusion Energy Division safety program implements the $Y-12$ safety program with procedures specific to controlled thermonuclear reaction (CTR) experiments.

\subsubsection{Department of Energy Safety Requirements}

The Department of Energy (DOE) safety requirements are stated in D0E Manual 0550, "Operational Safety Standards." All phases of the EBT-II project will be carried out in compliance with these requirements.

\subsubsection{Preparatory Safety Activities}

A formal Safety Analysis Report will be prepared and submitted to the $Y-12$ Central Safety Committee and the DOE Oak Ridge Operations office. They must review and approve the report before operation can begin.

Prior to initial operation, a detailed check of the experiment will be performed to verify that all equipment is in good operating order, that all controls and instrumentation are operating properly, and that the field installation has been done correctly. A committee of qualified technical personnel from outside the EBT-II operating group will review the engineering design from a safety standpoint and witness the check of the experiment.

\subsection{SAFETY PRECAUTIONS}

\subsubsection{Industrial Safety}

Al1 equipment will be fitted with safety devices and/or protective guards to prevent operator injury. Piping, pressure vessels, and electrical work will conform to applicable codes and standards. (Union Carbide Corporation General Design Criteria Y-EF-538 specifies 13 standards applicable to electrical systems.)

Any hazardous electrical areas will be interlocked with the entrance key captured on the master control pane1. Removal of this key shuts down the machine. Also, an emergency trip control which shuts 
down the 13.8-kV breakers leading to the power supplies will be under the control of the operating engineer.

Operations involving liquefied gases will use the procedures, protective clothing, and equipment developed for handling cryogenic liquids. Hydrogen flammability hazards will be controlled by established procedures.

\subsubsection{Microwave Safety}

The device is designed to totally contain the injected microwave energy. The vacuum vessel metalto-metal seals are of special design to ensure microwave containment as well as vacuum integrity. All openings for diagnostic access and vacuum pumpout will have traps to contain the microwaves at the injected frequencies.

All acccss doors through the biological shield around the device will be interlocked with the microwave power supplies. The interlock system will be redundant and designed such that all access doors must be locked and the keys inserted in a pancl in the control room before the microwave power supplies can be energized.

\subsubsection{Seismic Considerations}

The new portions of the facility will be designed in accordance with the seismic design criteria for DOE facilities to prevent unacceptable hazards to persons or property.

\subsubsection{Fire Protection}

Building 9201-2 is equipped with automatic sprinkler systems for protection of the building and equipment. Fire alarm boxes and sprinkler annunciator alarms are connected through the $Y-12$ Plant fire alarm system to the fire and guard headquarters and to the plant emergency control center, which is manned at all times.

In addition to the fire alarm system, all fire doors and alarms associated with the water distribution system are monitored by the plant monitoring system, which provides information on the exact location of an alarm and on planned procedures in a matter of seconds. (The system is presently being computerized and expanded to include the firm alarm boxes and sprinkler annunicator alarms for all ORNL-occupied buildings in $Y-12$, including B1dg. 9201-2.)

The Y-12 Plant Emergency Squad, consisting of 13 persons, responds to all alarms with a pumper, a fully equipped emergency truck, and an ambulance. Another pumper and a $\mathrm{CO}_{2}$ truck serve as backup. Personnel and equipment from ORNL, the Oak Ridge Gaseous Diffusion Plant, and the City of Oak Ridge respond to larger emergencies under mutual aid agreements.

\subsection{RADIOLOGICAL SAFETY}

The biological shielding for E⿱一土'-11 is based on a dose rate of $0.25 \mathrm{mrem} / \mathrm{hr}$, which is within the limits required for unrestricted occupancy. The radiological hazards have been determined by approximate methods based on worst case assumptions. A detailed analysis using modern computer codes may permit a reduction of the shielding requirements. The EBT-II experiment will use hydrogen routinely and will only use deuterium after a major hazards review and incorporation of necessary protection in the core plasma. 


\subsubsection{Hydrogen Plasma}

The main source of radiation with an ionized hydrogen plasma will be that due to bremsstrahlung from hot electrons in the annuli striking the aluminum side walls of the cavities. All other sources will be much lower in either energy (less than $100 \mathrm{keV}$ ) or intensity and will therefore be rapidly attenuated in any shielding adequate for the hot electron bremsstrahlung.

In the present design, the main operating console is nearer the machine than any other point in the control room; therefore, the dose rate is determined at this point, which is approximately $11 \mathrm{~m}$ from the center of the torus. The calculations assume a point source located at the center of each cavity; however, the geometrical factor which increases the effective shielding is ignored, as are the shielding effects of the machine components and supporting structure.

In determining the heat input to the superconducting coils, a maximum of 1 MW of microwave power was assumed to dissipate in the electron annuli and be converted to bremsstrahtung in the cavity liner with a conversion efficiency of 2.9\%. A similar assumption will be made here with the corresponding result that the biological shield will have a wide margin of safety. Also, the photon energy spectra of Edelsack et al. ${ }^{1}$ are used where the maximum electron temperature is taken to be 2 MeV.

The contribution to the dose rate due to the bremsstrahlung can be reduced to less than 0.25 $\mathrm{mrem} / \mathrm{hr}$ by a combination shield which consists of a shield around the cavities of $3.8-\mathrm{cm}-\mathrm{thick}$ uranium plus an enclosing concrete wall $61 \mathrm{~cm}$ thick. This is calculated by numerically integrating the 2-MeV bremsstrahlung spectrum with and without the attenuation produced by the shielding. ${ }^{2}$

\subsubsection{Deuterium Plasma}

Current plans do not call for the use of deuterium in EBT-II. If plans change, neutron shielding will be required for personnel safety. In addition, shielding will be required for some machine components, such as the superconducting coils.

\section{REFERENCES}

1. E. A. Edelsack, W. E. Kreger, W. Mallet, and E. Scofield, Health Phys. 4, 1-15 (1960).

2. E. P. Biizard, "Nuclear Kadiation Slitelding," in Nuclear Engino.pring Handbook, McGraw-Hill, New York, 1958. 


\section{THIS PAGE}

\section{WAS INTENTIONALLY LEFT BLANK}


5. BRIEF ANALYYSIS OF ENVIRONMENTAL IMPACT

The EBT-II facility will be a major upgrade of a similar existing facility; thus, its primary environmental impact will consist of increased requirements in electrical power generation and distribution and in the disposal of waste heat.

The proposed installation will require about $31 \mathrm{MW}$. This amount is available at the EBT-II site (Bldg. 9201-2 of the $Y-12$ Plant). Since the power to the project will be interruptible, the project will have no significant effect on power generating reserves in the area.

Waste heat will be dissipated through existing water-to-air cooling towers. The total waste heat from EBT-II represents about 50\% of the design capacity of the cooling towers. The fogging resulting from disposal of the waste heat is not considered significant.

There will be no visual impact. The device and most of the supporting systems will be inside Bldg. 9201-2. The cooling towers and power supplies to be used are screened from public view by intervening structures.

No material of strategic importance will be used. Most of the construction materials could be recovered after several years of operation.

A biological shield around the device will limit the radiological emanation from the device to an acceptable level (as defined by Department of Energy standards). 
THIS PAGE

\section{WAS INTENTIONALLY \\ LEFT BLANK}


APPENDIX A

STABILITY THEORY 
THIS PAGE

\section{WAS INTENTIONALLY LEFT BLANK}


The basic premise of EBT devices is that plasma currents produced by microwave heated, hot electron annuli can provide macroscopic stability for plasma confinement in a steady-state bumpy torus. Both: theoretical studies of the principle and experiments on EBT-I in the early 1970's have demonstrated the existence of a macroscopically stable plasma confinement regime. On the basis of the same theoretical considerations, EBT-II is also projected to be macroscopically stable. In order to improve our understanding of the requirements for macroscopic stability, MHD modes have been investigated in considerable detail, both analytically and numerically. Other drives for instability, including microinstabilities, have also been investigated to a lesser extent. In this appendix, the results of these theoretical stability considerations are summarized.

A comparison of the parameters of EBT-I and those projected for EBT-II yields the interesting result that most of the dimensionless parameters which influence stability change little throughout the sequence from EBT-I to EBT-II, and also to the projected EBT reactor. In particular, the following dimensionless parameters are constant or change in the direction of increased stability:

1. annular beta,

2. ratio of gyroradii to various scale lengths,

3. field errors,

4. aspect ratio,

5. ratio of cold to hot electron density,

6. ratio of electron to ion temperature,

7. ratio of perpendicular to parallel temperature,

8. ratio of electron plasma frequency to cyclotron frequency, and

9. ratio of ambipolar potential to temperature.

Insofar as stability is concerned, the most significant changes between EBT-I and EBT-II are in the toroidal core beta, which is projected to be approximately $10 \%$ compared to $0.33 \%$ to $0.5 \%$ in EBT-I, and, to a lesser extent, in collisionality. Present theoryl predicts that macroscopic stability can be obtained for beta of the toroidal core plasma ( $\beta_{\text {core }}$ ) comparable to that of the hot electron ring beta $\left(\beta_{\text {annulus }}\right)$ (e.g., 50\%). The approximate constancy of many of the dimensionless parameters is very encouraging in view of the positive results obtained experimentaliy on EBT-I.

The sources of free energy for instability which are presenl in EBT devices are:

1. finite beta $\left(j_{1}\right.$ or $\left.\nabla p\right)$,

2. parallel current,

3. collisions,

4. trapped particles,

5. centrifugal forces associated with equilibrium $E \times B$ rotation,

6. lossy regions of velocity space, and

7. anisotropy.

Only the more important drives for instability have been investigated theoretically in any detail, and in the following sections the calculations which have been carried out are reviewed. Inciuding all the above drives simultaneously in a full electromagnetic stability analysis remains to be performed.

The potential instabilities for EBT can be broadly classed as drift waves or drift waves coupled to other waves. The simplest forms of drift waves are MHD interchanges and ballooning modes. This analysis apparently contains the dominant drives for stability/instability and allows insight into those radial profiles which are stable.

This analysis is followed by a brief discussion of the effect of parallel current on MHD stability and a simple stability condition is derived. The motivation for this analysis is provided by field 
error measurements on EBT-I (see Appendix B). While it was shown that the net parallel current can easily be nulled, it was also possible to enhance the parallel current. The main interest derives from the possibility that by inducing field errors and parallel current, the general class of drift instabilities may be studied.

These low frequency, MHD instabilities apply to the toroidal core plasma. Vlasov treatments are required to justify this analysis and to study the stability of the hot electron annuli. Thus, these calculations briefly summarize and illustrate the types of density and temperature profiles required for the simultaneous stability of the toroidal core plasma and the hot electron rings. Finally, some recent calculations are discussed which take into account the other drives for instability mentioned earlier, such as trapped particles and collisions.

\section{A. 1 MAGNETOHYDRODYNAMICS}

MHD analysis presently provides the most geometrically detailed method of studying the stability properties of the toroidal core plasma. The model used ${ }^{2}$ is slightly nonstandard in that the hot electron rings are regarded as rigid. That is, the hot electron ring pressure and current are regarded as fixed equilibrium quantities, while the toroidal core plasma currents and pressures are allowed to deviate from the equilibrium values. Thus, for example, the total current is written

$\vec{j}_{\text {total }}=\vec{j}_{e}+\vec{j}+\vec{j}$,

where $\vec{j}_{e}$ is the hot electron ring current, $\vec{j}$ is the toroidal core equilibrium current, and $\delta \vec{j}$ is the perturbed toroidal plasma current.

The formalism² developed for the rigid annulus is applied in detail to a bumpy cylinder (a bumpy torus in which toroidal effects are neglected). The formalism is sufficiently close to that of standard $M H D^{3}$ that the stability criteria can be generalized to include toroidal effects and go over smoothily. ${ }^{4}$

In the axisymmetric case, it is convenient to use cylindrical coordinates $(r, \theta, z)$ as well as flux coordinates $(\psi, \theta, \ell)$. Here $\psi$ is the flux function $(B=\nabla \psi \times \nabla \theta)$, which is constant along field lines, and $\ell$ is arc length along field lines. The sufficient condition for stability reduces to

$$
\int \frac{d \ell}{B}\left[\frac{1}{\mu_{0}}\left(\frac{1}{r} \frac{\partial X}{\partial \ell}\right)^{2}+\frac{\partial p}{\partial \psi} D X^{2}\right]+\frac{\gamma p}{U+\mu_{0} \gamma p \int\left(d \ell / B^{3}\right)}\left(\int \frac{d \ell}{B} D X\right)^{2}>0
$$

where

$$
\begin{aligned}
& x=r B \xi \psi, \\
& \xi \text { is the piasma aispiacement, } \\
& D=-\left(\frac{\partial \ln B}{\partial \psi}+\frac{\kappa}{r B}+\frac{\mu_{0}}{B^{2}} \frac{\partial p}{\partial \psi}\right),
\end{aligned}
$$

and the curvature $\kappa$ is given by $k=\hat{e}_{\psi} \cdot \partial \hat{b} / \partial \ell$, where $\hat{e}_{\psi}$ and $\hat{b}$ are unit vectors in the $\psi$ - and $B-d i r e c-$ tions, respectively, and $\gamma$ is the ratio of specific heats. Here $U \equiv \int d \dot{l}, B$.

The expression given here for $D$ is formally the same as that obtained in the standard case. ${ }^{3}$ The

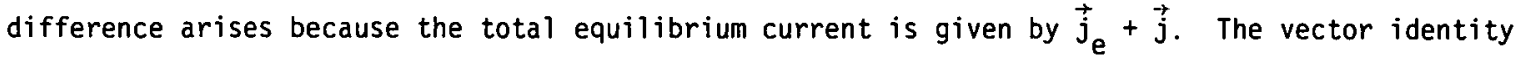

$\nabla \ln B=\vec{\kappa}+\frac{1}{B^{2}} \vec{B} \times(\nabla \times \vec{B})$ 
when combined with $\mu_{0}(\vec{j} e+\vec{j})=\nabla \times \vec{B}$ and $\vec{j} \times \vec{B}=\nabla_{p}$, yields

$\frac{\mu_{0}}{B^{2}} \frac{\partial p}{\partial \psi}+\frac{\partial \ln B}{\partial \psi}=\frac{1}{r B} \kappa-\mu_{0} j_{e} / B$.

Thus the driving term for the instability can be written

$D=\mu_{0} j_{e} / B-2 \kappa /(r B)$

Thus, the usual drive for ballooning modes (curvature) is modified by the hot electron ring current.

Ballooning modes tend to concentrate (or balloon) in regions of negative curvature. It is converient to study these modes by using the Euler equation,

$\frac{1}{\mu_{0}} \frac{\partial}{\partial \ell}\left(\frac{1}{r^{2}} \frac{\partial X}{\partial \ell}\right)+\left(\Lambda-\frac{\partial p}{\partial \psi} D\right) X=\frac{D \gamma p}{U+\mu_{0} \gamma p \int\left(d \ell / B^{3}\right)} \int \frac{d \ell}{B} D X$.

The procedure is to calculate numerical equilibria and then use a shooting code to determine the sign of the lowest eigenvalue $(\Lambda)$ on each of a closely spaced grid of field lines. If $\Lambda>0$ on all field lines, stability is assured.

The stability results will, of course, depend upon the details of the pressure profile. To gain insight into the type of profile which is likely to be stable, it is useful to examine perturbations for which $\partial X / \partial \ell=0$. These flutelike modes have a simple analytic expression for stability:

$\left(p^{\prime}+\gamma p \frac{u^{\prime}}{U}\right)\left(U^{\prime}-\mu_{0} p^{\prime} \int \frac{d \ell}{B^{3}}\right)>0$.

It will be noticed that for $p^{\prime}=\partial p / \partial \psi \sim \partial p / \partial r<0$, the first factor governs stability if $U^{\prime}=$ $\partial U / \partial \psi>0$, while the second factor governs stability if $U^{\prime}<0$. We also note that if $p^{\prime}=0$, then stability is indicated.

The hot electron annuli produce minima in $B$ and maxima in $U$, as indicated in Fig. A.1. In the central portion of the plasma where $d U / d r \sim U^{\prime}>0$, the stability condition for the flutelike modes is

$p^{\prime}+\gamma p \frac{U^{\prime}}{U}>0$

which yields as a marginal stability condition

$\mathrm{pu}^{\gamma}=$ const .

In the outer portion of the hot electron rings, $U^{\prime}<0$, so that here the stability condition reduces to

$U^{\prime}-\mu_{0} p^{\prime} \int \frac{d \dot{x}}{B^{3}}<0$

or

$$
\left|p^{\prime}\right|<\frac{\left|U^{\prime}\right|}{\mu_{0} \cdot \int_{B^{3}}^{\frac{d \ell}{2}}}
$$




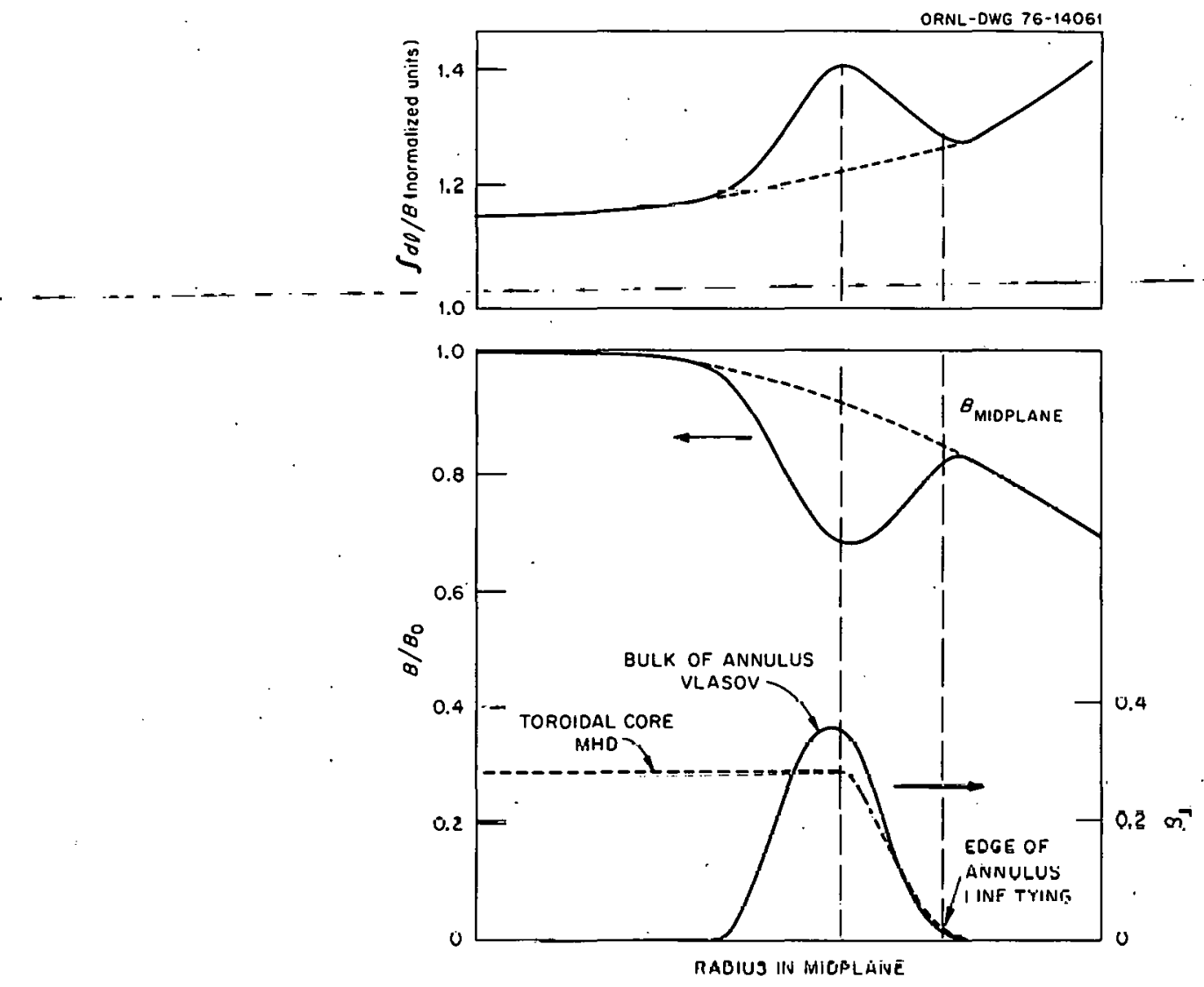

Fig. A.1. Effect of hot elertron annuli on $B$ and $U$. 
If these stability conditions are violated for the flutelike modes, then one can guarantee that the more stringent stability requirements for the ballooning modes will not be satisfied.

In the ballooning mode stability calculations carried out to date, no serious attempt was made to optimize the shape of the toroidal core pressure profile. Instead, the toroidal core pressure was taken to be constant $\left(p^{\prime}=0\right)$ in the region of positive $U^{\prime}$ and to fall to zero in the outer region of the rings where $U^{\prime}<0$. In this outer region, the pressure was taken to be a simple poiynomial in $\psi$, $p \sim\left(\psi-\psi_{1}\right)^{2}\left(\psi-\psi_{2}\right)^{2}$

The results of these calculations are indicated in Fig. A.2. At high values of the hot electron annulus beta, the toroidal core beta can be comparable to the annular beta before MHD stability is no longer guaranteed. Annuli of long axial extent produce longer regions of minimum- $B$, and somewhat larger values of toroidal beta can be achieved, as indicated in Fig. A.2. At high beta, the stability boundary is relatively insensitive to the width of the hot electron rings.

At low values of beta, the stability boundary is sensitive to the width as well as the length of the rings. Notice that the stability curves pass through zero at low but finite values of annular beta. This is just the value required to produce well defined minima in $B$. For annular beta below this value; the toroidal core plasma should be unstable. This value of annular beta is associated with the experimentally observed transition between the noisy C-mode and the quiet $T$-mode of operation.

It is encouraging that the pressure profiles assumed for the stability calculations are in qualitative agreement with the densities and temperatures obtained by the more recent neoclassical transport simulations. Calculations are in progress to determine the optimum toroidal core pressure profiles which maximize the toroidal core beta. Preliminary indications are that they will not differ significantly in shape from those obtained from the simulation model.

\section{A.2 A SIMPLE ESTIMATE OF THE EFFECT OF PARALLEL CURRENT}

The parallel current was first observed experimentally and is apparently produced by small field errors $\left(\delta B / B \sim 10^{-4}\right.$ ) which can be globally nulled (or enhanced) by simple corrector coils. The total current is small compared to the familiar ohmic heating current in tokamaks [e.g., reduced by a factor of $10^{-3}$ from that of the Oak Ridge Tokamak (ORMAK), which had a comparable cross-sectiorial area). Parallel current is a well known drive for instability (e.g., kinks in a tokamak). While the radial profile of the parallel current is presently unknown, it is reasonable to assume that if the total current is small, the local currenl density is also small. IInder these circumstances the usual resonant condition for kinks $(\vec{k} \cdot \vec{B} \cong 0$ or $m-n q \cong 0)$ yields a flutelike condition. Thus, we are led to include parallel current as a perturbation to the analysis for flutelike and ballooning modes discussed in the previous section.

In examining the modified energy principle, ${ }^{2}$ we find that introducing parallel current leads to a single additional term in $\delta w:$

$\delta W=\frac{1}{2} \int d^{3} \times\left[\frac{1}{\mu_{0}} Q^{2}+\vec{j}_{\|} \cdot(\vec{\xi} \times \vec{Q})+\ldots\right]$.

Here $\vec{Q}$ is the perturbed magnetic field, $\vec{j}_{\|}$is the equilibrium parallel current, and $\vec{\xi}$ is the plasma displacement. In specifying equllibrid, the toroidal current $\left(2 \vec{j}_{\|}\right)$is an independent quantity in addition to the pressure tellsor for the hot electron rings and the scalar pressure for the toroidal core plasma. Thus, $\vec{j}$ can be associated with the hot electron rings and/or the toroidal core plasma. 


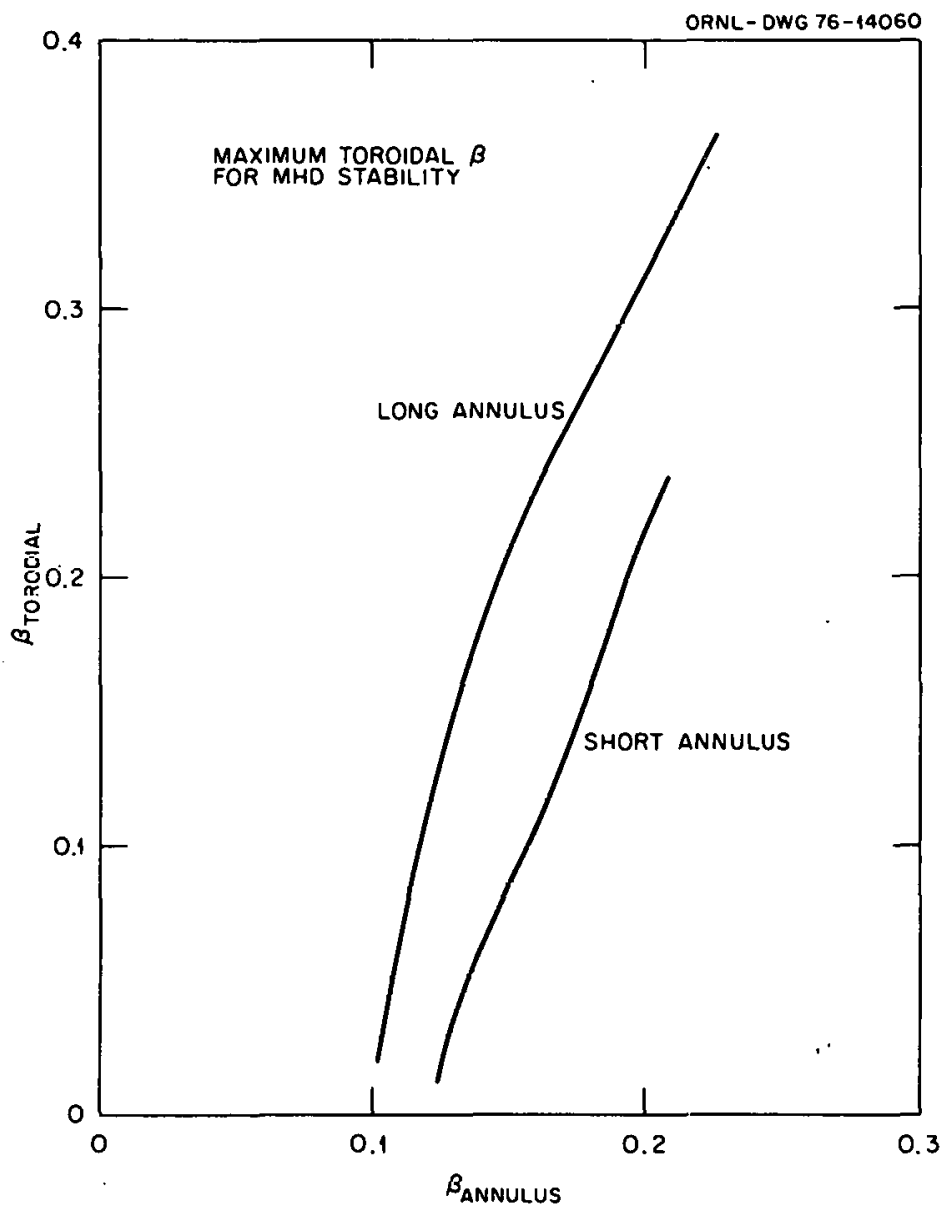

Fig. A.2. Maximum stable toroidal beta as a function of Bnnnilus for a long annulus and for a shnit nmulus. 
The new term introduced by the parallel current can be combined with other terms in sw in a variety of ways to produce slightiy different stability criteria. Here, a specific example is given which is quite simple:

$\frac{1}{\mu_{0}} Q_{\perp}^{2}+\vec{j}_{\|} \cdot(\vec{\xi} \times \vec{Q})=\frac{1}{\mu_{0}}\left|\vec{Q}_{\perp}-\frac{1}{2} \mu_{0} \vec{j}_{\|} \times \vec{\xi}\right|^{2}-\frac{1}{4} \mu_{0} j_{\|}^{2}\left|\overrightarrow{\xi_{L}}\right|^{2} \cong \frac{1}{\mu_{0}} Q_{1}^{2}-\frac{1}{4} \mu_{0} j_{\|}^{2}\left|\xi_{\perp}\right|^{2}$.

Now insert the $\xi_{\theta}$ which minimizes $\delta W$ in the absence of parallel current $\left[\xi_{\theta} \sim(1 / m) \xi_{\psi}\right]$, and obtain

$\int \frac{d \ell}{B}\left[\frac{1}{\mu_{0}}\left(\frac{1}{r} \frac{\partial X}{\partial \psi}\right)^{2}+\left(\frac{\partial p}{\partial \psi}-\frac{\mu_{0}}{\psi} \frac{j_{\|}^{2}}{r^{2} B^{2}}\right) x^{2}\right]+\frac{\gamma p}{U+\gamma p \int\left(d \ell / B^{3}\right)}\left(\int \frac{d \ell}{B} D X\right)^{2}+0\left(\frac{1}{m^{2}}\right)+0\left(\frac{j_{\|}}{m}\right)>0$.

For the purposes of this specific example, it is sufficient to examine the flutelike modes $(\partial X / \partial \ell=0)$, although the Euler equation is readily obtained. It is found that in place of Eq. (7), we obtain

$\left(p^{\prime}+\gamma p \frac{U^{\prime}}{U}\right)\left(U^{\prime}-\mu_{0} p^{\prime} \int \frac{d \ell}{B^{3}}\right)-\frac{1}{4} \mu_{0} \int \frac{d \ell j_{\| l}^{2}}{r^{2} B^{3}}>0$

as a condition for stability. This condition shows explicitly the destabilizing effect of parallel. current. In terms of marginal stability, it lowers the permissible magnitude of the pressure gradient.

since the pressure profiles were not known to high precision in EBT-I, it is difficult to obtain accurate estimates of the threshold for parallel current to induce instability. Qualitatively, one expects that if the pressure gradient rises above those tolerable for stability, then the system will relax rapidly to marginal stability. A crude estimate of the current density required in EBT-I to induce instability is $10 \mathrm{~A} / \mathrm{cm}^{2}$. This is about a factor of ten times the average current density (total current/total cross-sectional area) obtained from experiment. Thus, it must be assumed that the parallel current is concentrated within approximately $10 \%$ of the total cross section.

Since the distribution of the parallel current density produced by field errors is unknown, it may even be hypothesized that it is in alternate directions in different regions of the plasma. Thls can produce high current densities locally. However, the higher the local current density, the shorter its spatial "wavelength." For such high oscillatory parallel current densities, there will be nontrivial shear in the magnetic field, which shuuld have some stabilizing influenre. To analyze such a complicated hypothesis accurately would probably require much more effort than is presently warranted.

\section{A.3 STABILITY OF THE HOT ELECTRON RINGS}

The stability of the hot electron rings requires Vlasov treatment because the characteristic poloidal drift frequencies can be comparable to or greater than the ion cyclotron frequency. 5 Recent calculations ${ }^{6}$ indicate that the rings are stable provided the ratio of cold to hot electron density 15 sufricienlly high (c:g., 10). Those slah mndel calculations are presently restricted to stability of the hot electron rings since the ion temperature was set to zero. A similar Vlasov model, inciuding ion temperature, has been used to justify the rigid annulus MHD model discussed earlier.

While more work clearly needs to be performed in this area, some conclusions can be drawn from the results to date. Apparently there is a symbiotic relation between the toroidal core plasma and the hot electron rings; the toroidal core plasma provides the cooler electrons to stabilize the rings, while 
the hot electron rings provide the minimum-B necessary to stabilize the toroidal core plasma. An example of how the densities and temperatures can be arranged for stability is shown in Fig. A.3.

While the figure illustrates that the region inside the outer edge of the hot electron rings is stable, it also shows that the cold plasma outside the hot electron rings is potentially unstable. Within the context of MHD, 1 ine tying can stabilize this region. Nonetheless, there are a number of instabilities on the outside which cannot be totally stabilized, and this region is observed experimentally to be noisy. Although in EBT-I this region occupied a rather large fraction of the total vululle, there appears to be no stability criterion which would preclude reducing the fraction of the total volume considerably. However, some small volume of noisy cold plasma may be required for stability, and such a region may also be useful in screening impurities from the central portion of the plasma, as observed in EBT-I.

\section{A. 4 DISSIPATIVE AND OTHER INSTABILITY DRIVES}

A s imple buulice-averaged dritt kinetic model' was used to investigate the electrostatic stability properties of low frequency drift waves in the frequency orderings appropriate for the EBT device. Care has been taken to model (at least in an elementary way) the features which distinguish the physics of EBT from those of tokamaks: the large magnitude and velocity space dependence of the magnetic precessional frequency $(\Omega)$, the relatively small collisionality $\left(v_{j} / \Omega_{j}\right)$, the enhancement. of the cffective collision frequency for passing particles, and the closed nature of the field lines. The equations for the structure of modes along field lines have not yet been solved. Instead, the consequences of two assumptions were investigated. First, it was assumed that the variation of the potential had a much larger scale length than the length of a single sector and that the average of the potential around the torus vanished. In this case, the passing particles respond completely adiabatically to the drift wave. Second, the case of pure flute modes was investigated. Here the passing particles also had a nonadiabatic response. In each case, for normal gradients $\left[\omega_{*} / \Omega>0\right.$, where $\omega_{*}=\left(\ell T_{e} / m_{e} \Omega_{e}\right)(r / n)(d n / d r)$ is the electron diamagnetic drift frequency], instability was pussible for sufficiently large $\omega_{\star} / \ell \Omega$. The modes had a dissipative character in that the growth rates and the region of possible instability in $\omega_{\star}-\Omega$ space increased with decreasing collisionality. It was also found that the lidynitude and velocity space dispersion of $s$ are significant stabilizing influences, since in most cases the modes are completely stabilized for $\omega_{A} / p, \Omega<1$ For the caco of reveraed gradierils $\left(\omega_{*} / s<0\right)$, stabllizy is greatly enhanced, and growth rates decrease with decreasing $v / \Omega$.

Much additional analysis of the linear and nonlinear behavior will be necessary in order to assess whether these modes are of any real importance in scaling the FRT roncept to a reactor dcvicc. The results of the present study must be considered to be encouraging in that the modes can be stabilized by proper tailoring of the $\omega_{*}$ and $\Omega$ profiles.

Drift instabilities at higher frequencies have also been considered briefly, although no detailed calculations have been performed. The lower hybrid drift instability, which plays a role in high heta dcvices such as theld ulnches and reversed field pinches, appears not to be relevant for EBT. This is because EBT is a steady-state device in which both electrons and ions have the same $\vec{E} \times \vec{B}$ drift velocity. When the relative $\vec{E} \times \vec{B}$ drift between electrons and ions vanishes, the growth rate of the lower hybrid drift instability goes to zero, 8 and the real frequency becomes comparable to the ion cyclotron frequency. In this limit the lower hybrid drift instability goes over to a drift cyclotron or Mikhailovskii-Timofeev mode. The drift cyclotron mode requires that the real part of the frequency be closely tuned to a multiple of the ion cyclotron frequency $\left[\mathrm{e} . g .,\left(\omega_{r}-\Omega_{j}\right) / \Omega_{i} \leq \sqrt{m_{e} / m_{i}}\right]$. In machines having strong magnetic field gradients along the field lines, such as EBT or mirror machines, the mode can be stabilized by detuning of the particle resonances along the field lines. Much more work is needed in this area of high frequency drift instabilities. 


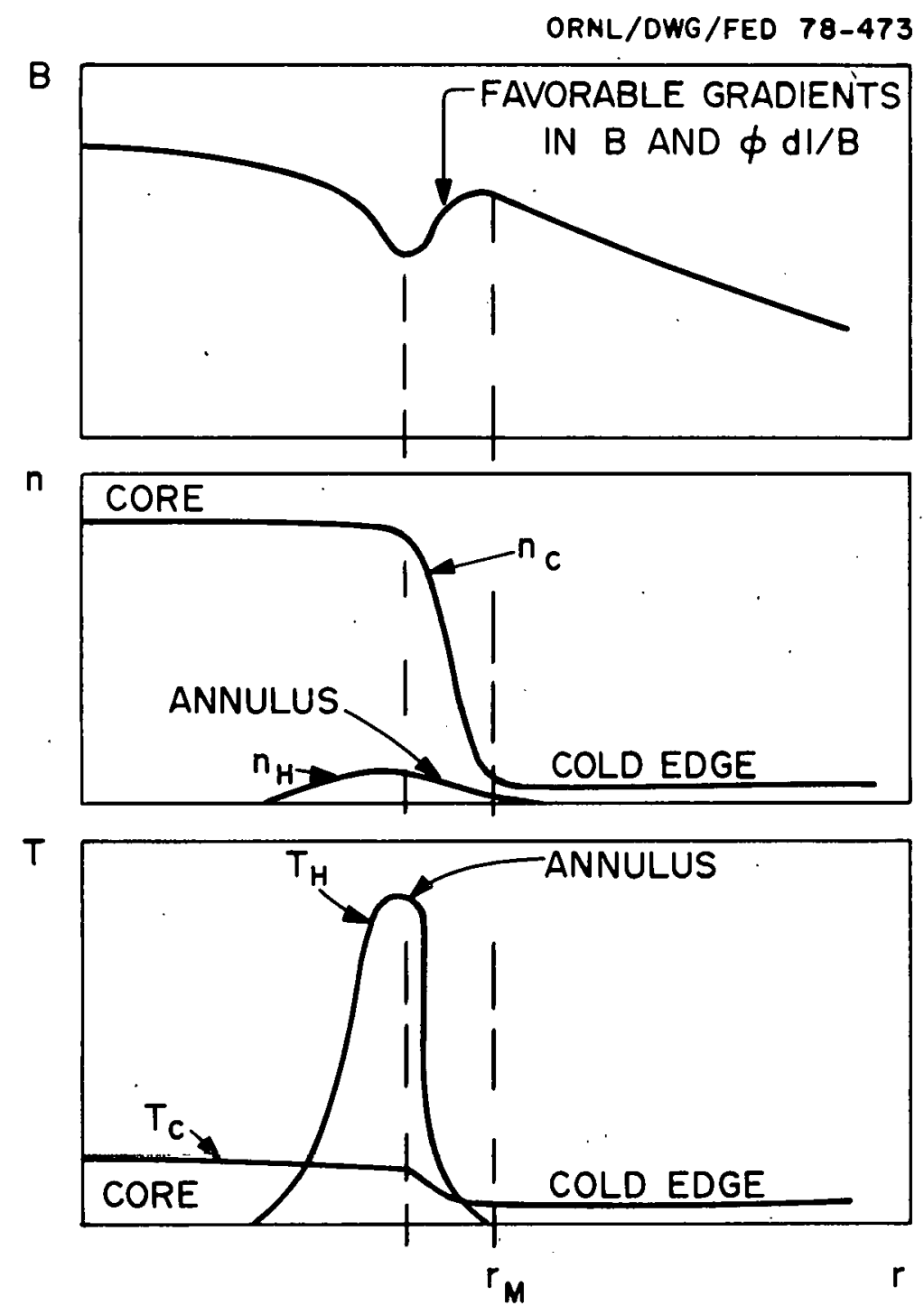

Fig. A.3. Comparison of densities and temperatures for annulus and toroidal core plasma. 
Plasma rotation due to the $\vec{E} \times \vec{B}$ drift in the ambipolar electric field is potentially a drive for instability, particularly for straight field line devices such as theta pinches. However, on the basis of MHD and estimates using a Vlasov model, terms involving curvature and gradients in $B$ are found to dominate the small destabilizing effects of $\vec{E} \times \vec{B}$ drift by a factor of about $10^{3}$. The centrifugal effects are therefore not expected to play an important role in EBT.

Estimates of the stability properties of any plasma depend upon details of equilibrium profiles. In making the above estimates, it has been necessary to assume that the equilibrium properties of EBT-II will be similar to those encountered in EBT-I.

\section{REFERENCES}

1. D. B. Nelson and C. L. Hedrick, Macroscopic Stability and B Limits in the ELMO Bumpy Torus, ORNL/TM-5967, Oak Ridge, Tennessee (December 1977); tọ be pụḅ]ịṣhed in Nụçl. Fuṣion.

2. J. L. Jöhnisón, R. M. Kulsrud, and K. E. Weimer, Mlasina Phiys. 11, 463 (1969).

3. I. B. Bernstein, E. A. Freeman, M. D. Kruskal, and R. M. Kulsrud, Proc. R. Soc. London Ser. A 244, 17 (1958).

4. D. B. Nelson and G. 0. Spies, Phys. Fluids 17, 2133 (1974).

5. G. E. Guest, C. L. Hedrick, and D. B. Nelson, Phys. Fluids 19, 871 (1975).

6. R. R. Dominguez and H. L. Berk, Phys. Fluids 21, 827 (1978).

7. D. B. Batchelor and C. L. Hedrick, A Preliminary Investigation of Trapped Particle Instabilities in EBT, ORNL/TM-6318, Oak Ridge, Tennessee (May 1978).

8. J. P. Freidberg and R. A. Gerwin, Phys. Fluids 20, 1311 (1977). 
APPENDIX B

DETAILED SUMMARY OF EBT-I EXPERIMENTAL RESULTS 
THIS PAGE

\section{WAS INTENTIONALLY LEFT BLANK}


From the point of view of a reactor design, a bumpy torus confinement geometry has many attractive features: symmetric magnet coils, large aspect ratio for convenient access and shielding, and the possibility of steady-state operation. However, even the most superficial examination of the unfavorable magnetic field curvature leads one to suspect the plasma would be MHD unstable. In 1971, a specific bumpy torus, 1 the ELMO Bumpy Torus (EBT-I), was proposed. It was designed to circumvent the MHD stability problem by using the diamagnetism of energetic electron annuli produced by electron cyclotron heating $(\mathrm{ECH})$ to modify the unfavorable magnetic curvature.

Construction on EBT-I was begun in 1972, and experiments were designed to examine three basic questions:

1. Can stable, diamagnetic electron annuli be generated by electron cyclotron heating in a bumpy torus?

2. If such annuli form, is the toroidal plasma confined stably by the resultant modification of the magnetic curvature?

3. Can the stable plasma equilibrium be sustained in steady state in a bumpy torus?

Experiments were begun in September 1973 and almost immediately, affirmative responses were found to each of the three fundamental questions. The evidence for these conclusions is presented in Sect. B.2.

When these proof-of-principle experiments were confirmed, a series of studies was initiated with the available heating power $(\approx 30 \mathrm{~kW}$ at $18 \mathrm{GHz})$ in order to document the plasma parameters in the stable, toroidally confined regime. It was soon determined that an extensive diagnostic development program was required, since the plasma parameters fell in a range which stressed the technological limits of the conventional measurement techniques. At the same time, simple estimates of confinement properties indicated that with higher applied power, a modest improvement in plasma temperatures could be expected, thus alleviating the diagnostic difficulty to some extent. Also, initial scaling of these properties with power would become possible.

As a consequence, experiments aimed at detailed studies of plasma parameters were initiated, with added flexibility resulting from a doubling of the available microwave power. Further, upon the addition of available microwave power sources at $10.6 \mathrm{GHz}$, independent control over the diamagnetism of the annular electron component was demonstrated; these sources were also added for routine use to the system.

With this configuration, after a technological upgrading of the entire diagnostic array, the plasma parameters in the stable, toroidal plasma were documented (see Sect. B.4). The relatively high density $\left(n_{e} \geq 10^{12} \mathrm{~cm}^{-3}\right)$, high temperature $\left(T_{e} \sim 300 \mathrm{eV}, T_{\mathfrak{i}} \sim 100 \mathrm{eV}\right)$, toroidally confined plasmas which were observed inspired additional theoretical effort. The resulting plasma models led to predicted plasma properties which, when extrapolated to higher magnetic fields and applied power, looked very attractive. The plasma models also suggested that a wider range of measurements should be undertaken, including a study of the influence of the ambipolar electric field, which is generated as a consequence of unequal loss rates of ions and electrons.

These measurements led to modifications in the theoretical models and to a deeper understanding of the fundamental confinement process in EBT-I (and its possible successors).

In this appendix, the experimental results from EBT-I's four years of operation are described. A more complete discussion of these results may be found in Ref. 2 .

\section{B. 1 PHYSICAL DESCRIPTION AND OPERATION}

The physical dimensions and operational parameters of EBT-I are listed in Table B.1. A layout of the facility is shown in Fig. B.l and a photograph of the machine in Fig. B.2. The magnetic field was 
ORNL / OWG 73-5045R4

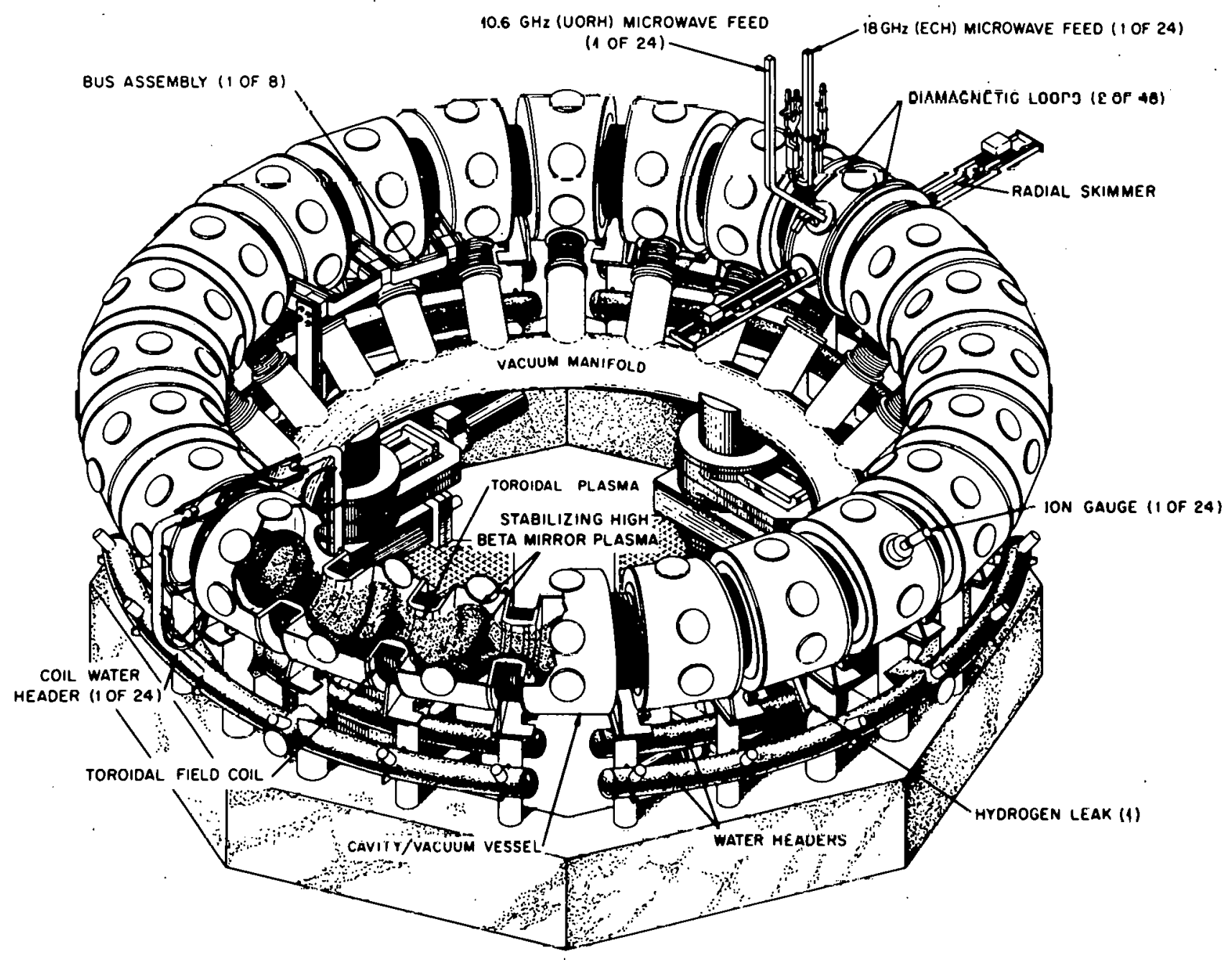

Fig. B.1. Schematic lavout of EBT-II. 


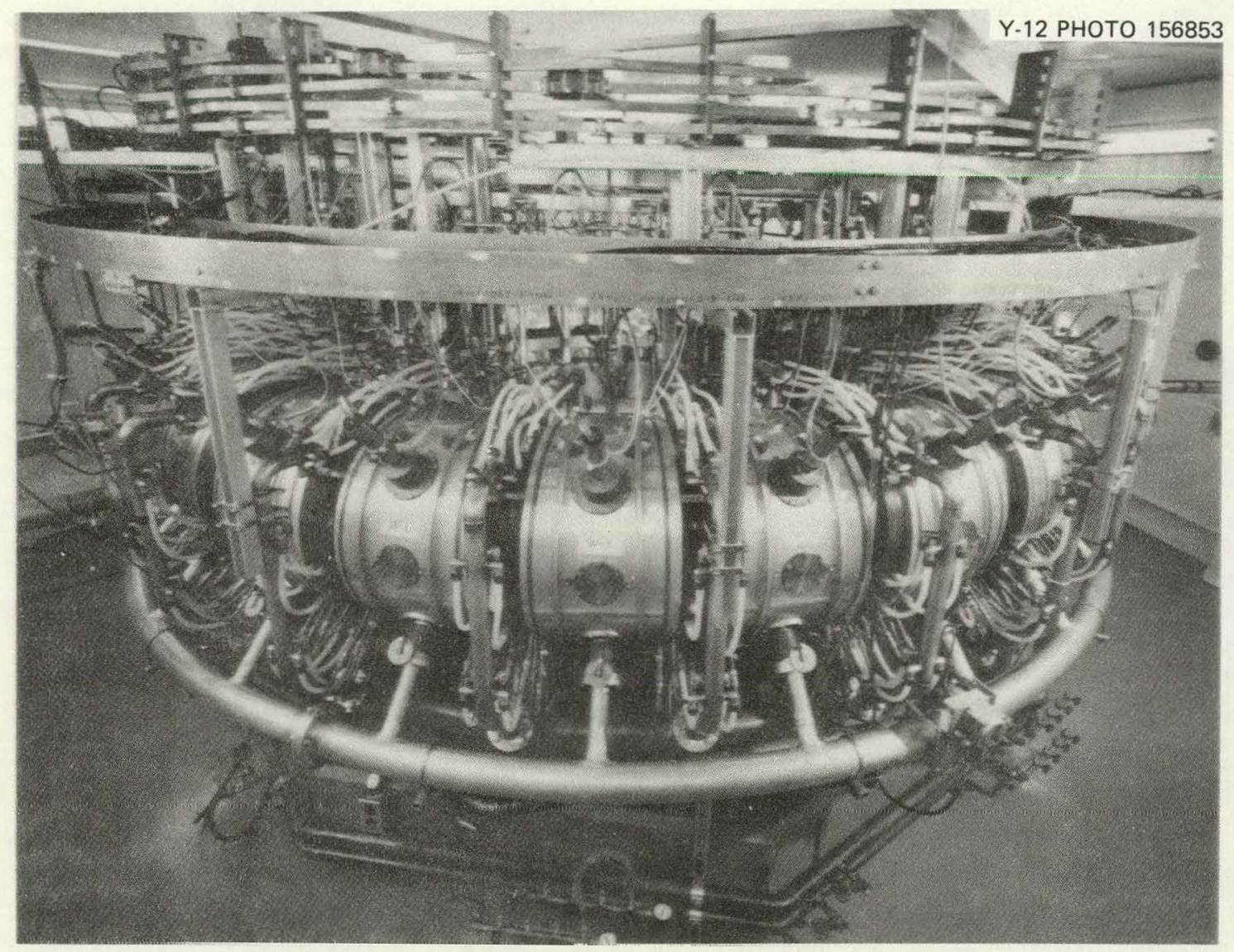

Fig. B.2. Photograph of EBT-I. 
Table B.1. Physical dimensions and operating parameters

\begin{tabular}{ll}
\hline Magnetic field & $6.5 \mathrm{kG}$ midplane, $13 \mathrm{kG}$ mirror \\
Magnetic field power & $10 \mathrm{MW}$ \\
Torus volume & $\cong 1351$ 1iters \\
Major radius & $150 \mathrm{~cm}$ \\
Aspect ratio & $\cong 8: 1$ \\
Microwave power (cW) & $60 \mathrm{~kW} \mathrm{cW}, 18 \mathrm{GHz}$ \\
ECH microwave source & $30 \mathrm{~kW} \mathrm{cW}, 10.6 \mathrm{GHz}$ \\
LORH microwave source &
\end{tabular}

produced by 24 magnetic coils arranged about a torus, each pair producing a 2:1 magnetic mirror ratio on the axis. The aluminum vacuum chamber in each toroidal bump was pumped into a toroidal vacuum manifold connected to four baffled, $25-\mathrm{cm}$ diffusion pumps operated with high quality "Santovac 5" pump 0i1. Each magnetic mirror sector was fed with microwave power at both $10.6 \mathrm{GHz}$ and $18 \mathrm{GHz}$ - the distribution system included four 18-GHz klystron amplitiers and three 10.6-GHz klystron amplifiers.

The power at each frequency was controlled by adjusting ine output of the low power reflex oscillator which constituted the source for the various amplifiers. Additionally, control of the power at $18 \mathrm{GHz}$ into each toroidal sector was provided through the control of a ferrite device which could be used to shunt any fraction of the power into a sector into a dummy load. These independent controls were designed into the system in case difficulties arose due to inadequate balance of power about the torus. Although this worry proved to be unfounded, the convenient remote power controls were of great assistance for diagnostic flexibility. The entire facility was contained in a 15-cm-thick lead shielded room to protect personnel from the hard $x$ rays produced by the energetic electrons in the annulus when they struck the walls.

A cross section of one toroidal section in the equatorial plane is shown in Fig. B.3. Both magnetic flux lines and surfaces of constant $|B|$ are also indicated. The magnitude of the magnetic field was selected so that cyclotron resonance was established for the applied frequency for bulk heating along surfaces approximately midway between the field minimum in the midplane and the maximum field, as illustrated in Fig. B.3. Under these conditions, because of the usual radial magnetic gradient in mirror geometry, surfaces were generated which had half the resonance field value at radii of $\cong 15 \mathrm{~cm}$. Electrons passing through these surfaces were then in resonance at the second harmonic of the electron cyclotron frequency and were found to be effectively heated to high energies - thus producing annuli of precessing energetic plasma at these radii. To control the energy density in these annuli, supplementary power was applied at a frequency $(10.6 \mathrm{GHz})$ which was at fundamental cyclotron resonance close to the locations of second harmonic resonance described above, as illustrated in Fig. B. 3 for $B_{00}=$ $0.45 \mathrm{~T}$. It was possible, therefore, to have a measure of independent control over the stored energy of the annulus (i.e., beta) and the bulk heating power and thus to provide a range of operating parameters not available from a single frequency source alone.

EBT-I was generally operated CW, and feedback control of the critical operational parameters was provided to ensure a constant steady state. To operate the device, the magnetic field was adjusted to the desired level and the operating gas, usually hydrogen (although deuterium, helium, nitrogen, argon, and even air were all used as operating gases), was fed into one of the toroidal bumps so that the pressure was raised to $\approx 10^{-5}$ torr. As the microwave power was raised, a plasma was formed and the gas recirculated to the walls until an equilibrium was reached between gas returning from the walls and losses out of the plasma. When this equilibrium was established, it was found that the ion gages in 


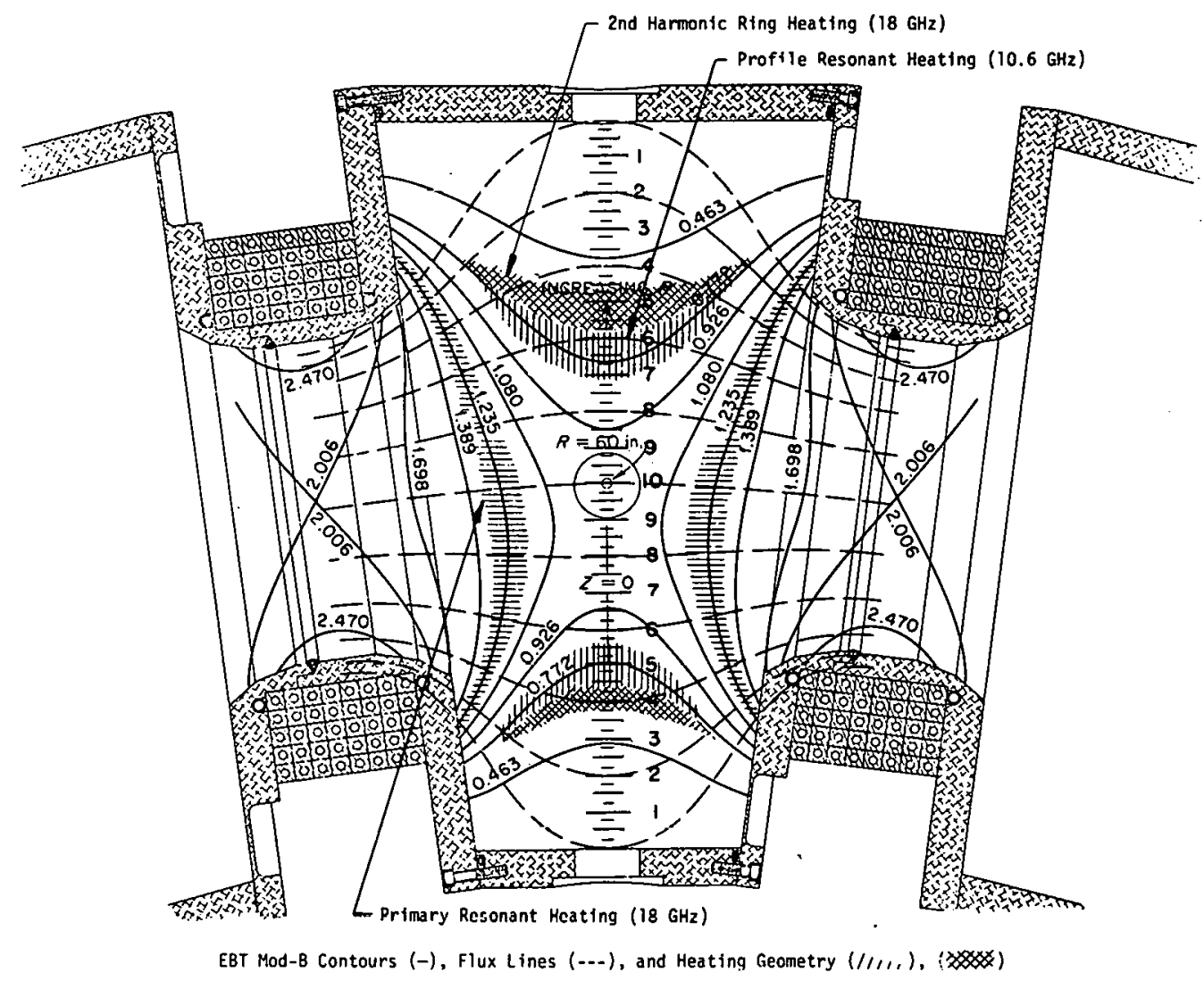

Fig. B.3. Section through EBT-I in the horizontal midplane. Solid lines indicate surfaces of constant $|B|$; dotted lines indicate flux 1 ines. Shaded areas are regions of cyclotron resonance for electrons when $B_{0}=4.55 \mathrm{kG}$. 
each sector read the same, indicating that the circulation of plasma about the torus had introduced equal losses to the walls in each sector, and the plasma was in equilibrium with these recirculated particles. The controlled gas feed input was, then, just a "makeup" gas for the small fraction of circulating atoms which managed to be pumped out of the device. The desired plasma conditions could then be established by adjusting the profile power at $10.6 \mathrm{GHz}$, the bulk heating power at $18 \mathrm{GHz}$, and the ambient gas pressure.

\section{B.2 EARLY EXPERIMENTS IN EBT-I}

\section{B.2.1 DEMONSTRATION OF VALIDITY OF EBT CONCEPT}

Confinement of plasma in a spatially and periodically modulated toroidal magnetic field (bumpy torus) previously received very little experimental attention because of anticipated MHD instability as a consequence of the unfavorable magnetic field gradients. In EBT, it was proposed that the toroidal plasma could be stabilized by generating poloidal plasma currents in steady state which would cancel the negative field gradients in each "bump" of the torus. These currents were expected to arise from the diamagnetism resulting from annuli of energetic electrons which, as was seen in mirror machine experiments at ORNL, are a consequence of electron cyclotron heating in this geometry.

Construction of the EBT facility was approved in 1972 and completed in September 1973. Initial experiments were begun with microwave power fed into only four toroidal bumps to examine the conditions required to establish stable, diamagnetic annuli in this novel toroidal configuration. On the very first day of experiments (September 13, 1973) finite stored energy in the four cavities was observed and found to decay stably when the power was interrupted (Fig. B.4).

There followed a period of intense activity in which the next critical question was addressed: does the diamagnetism of the hot electron annulus modify the magnetic curvature sufficiently to stabilize the toroidal plasma?

An affirmative response to this question was determined by examining the level of density fluctuations seen in a microwave interferometer as a function of stored energy $\left(W_{\perp}\right)$ contained in the electron annulus (Fig. B.5.). At high neutrai pressure and modest microwave heating power, $W_{L} \approx u$, out a significant average plasma density $\left(n_{e} l\right)$ was measured with the interferometer. The fluctuation ievel under these conditions was high, $\Delta(n \ell) /(n \ell) \approx 1$. This regime of operation was designated the $C$-mode (for cold plasma operation). As the neutral pressure was lowered, the stored energy in each sector increased (the energy in one particular sector is illustrated in Fig. B.5). ne fell slightiy as the feed gas was reduced, but the fluctuation level $\Delta(n \ell) / n \ell$ fell dramatically to almost thermal levels in optimum cases $\left(\Delta n \ell / n \ell \sim 10^{-3}\right)$ when $B \geq 0.1$, a value consistent with theoretical predictions of the requirements for stability of a toroidally confined, Maxwellian plasma. In this regime, designated T-mode (for toroidal plașa), the electron and ion temperatures were found to be relatively high. In Fig. B.5 this is illustrated by $30 \mathrm{eV}<\mathrm{T}_{i}<70 \mathrm{eV}$, determined from the measured energy distribution of charge exchange neutrals. These facts constituted a proof-of-principle demonstration of the EBT concept.

\section{B.2.2 MEASUREMENT OF THE LOCATION OF THE TOROIDAL PLASMA}

The physical size and location of the plasma were measured using several diagnostics. The loration of the diamagnetic annulus was determined by inserting a water cooled skimmer probe into one of the toroidal sectors from both the outside and the inside of the torus. The probe contained an ionization chamber which was sensitive to locally generated $x$ rays produced as the probe intercepted the energetic electrons in the annulus. In Fig. B.6, the observed current in the ionization chamber is shown as a 


\section{Sy opower \\ THERMONUCLEAR DIVISION}

DATA SHEET

Sept $13 / 73$

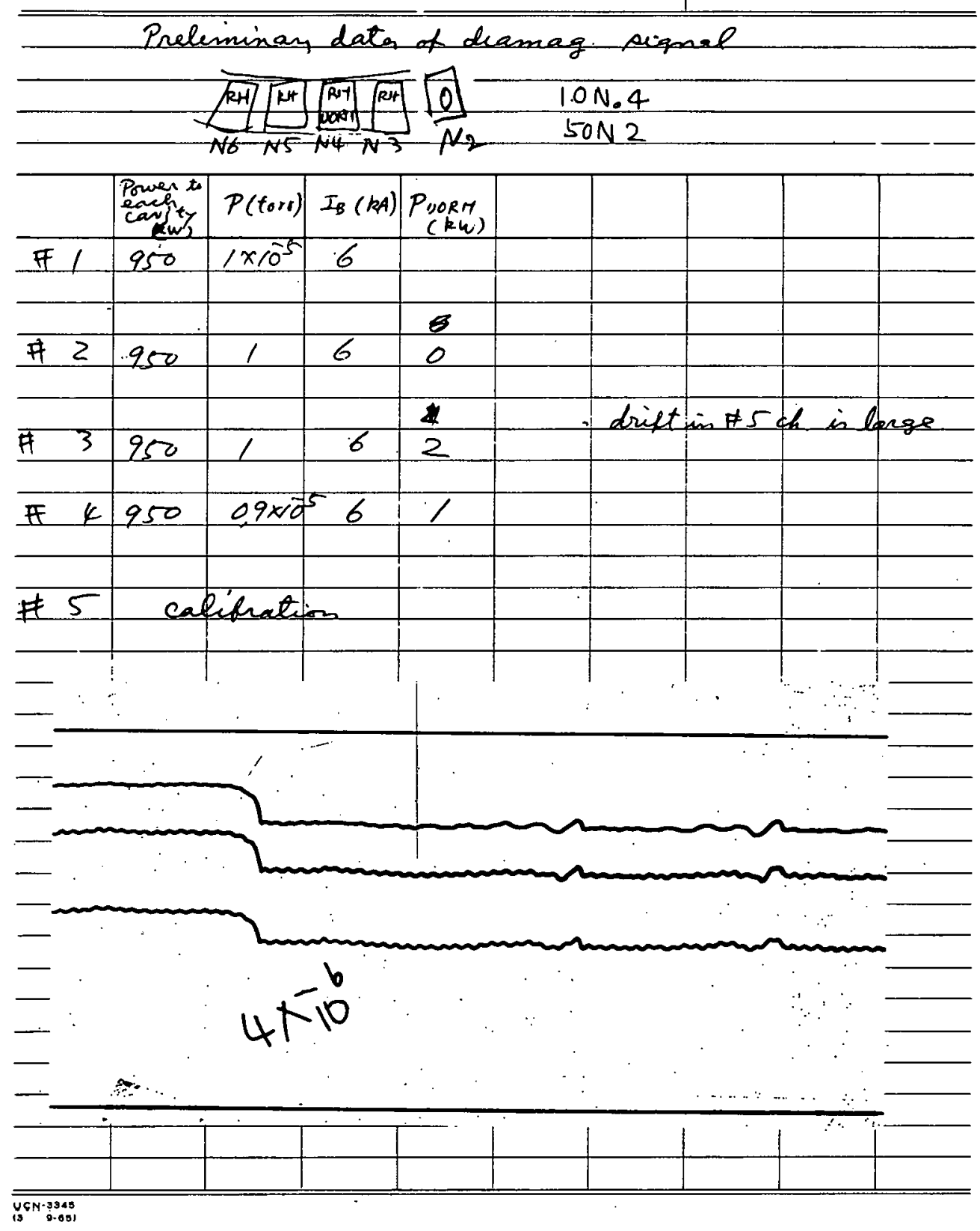

Fig. B.4. Data sheet of September 13, 1973 showing $950 \mathrm{~W}$ of 18-GHz power delivered to each of four toroidal sectors. At bottom, tracings from diamagnetic loops in three of the sections indicating decay of diamagnetism following turnoff of plasma. 


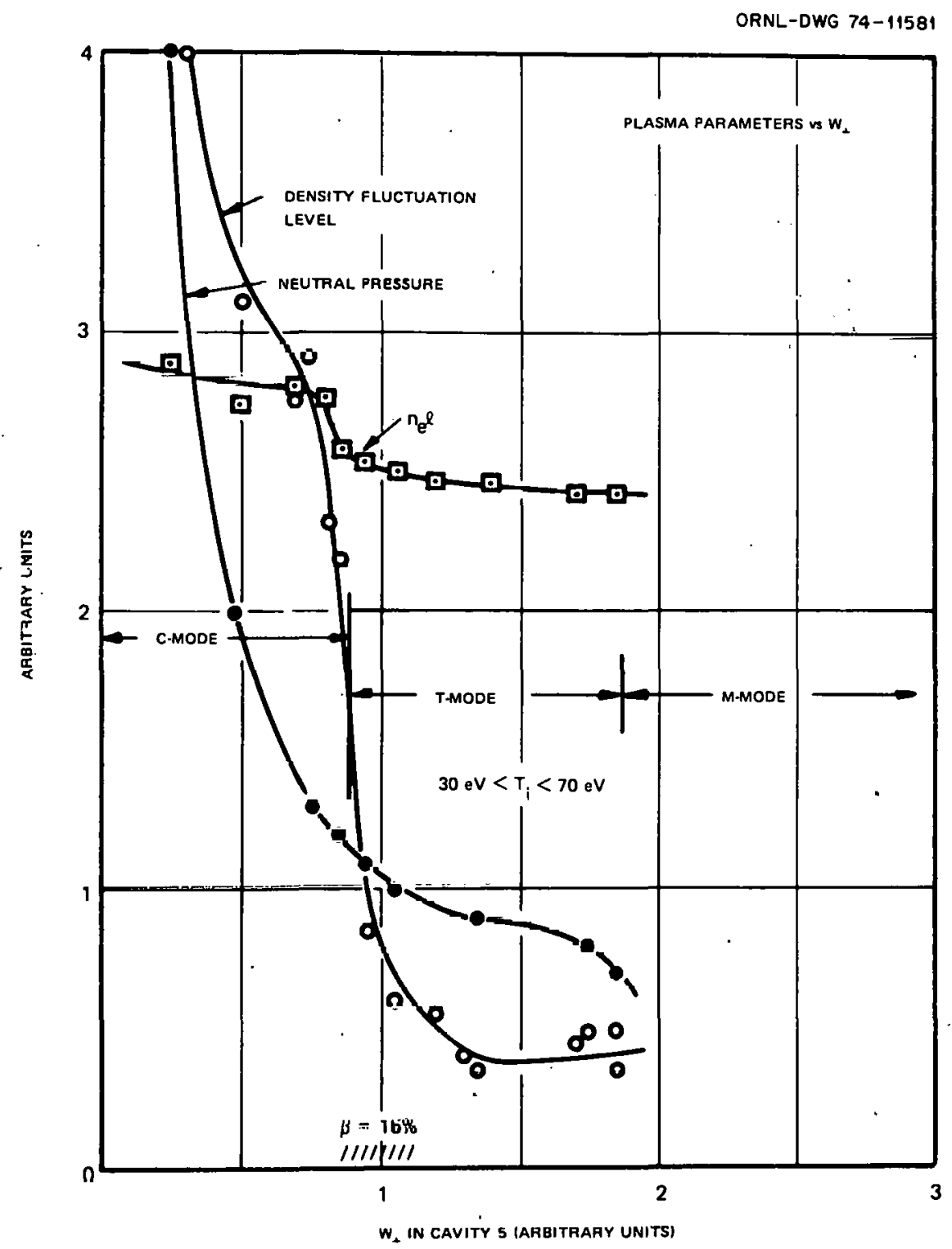

Fia. B.5. Plasma parameters męąured in EBT-I ae a function of observed stored energy $W_{1}$. Solid dots are for neutral pressure, open dots are for density fluctuation level, squares are for line density, $n_{e^{l}}$. 
ORNL/OWG/FED-78-337 SKIMMER PROBE ION CHAMBER CURRENT DIAMAGNETIC FLUX VS SKIMMER PROBE RADIUS

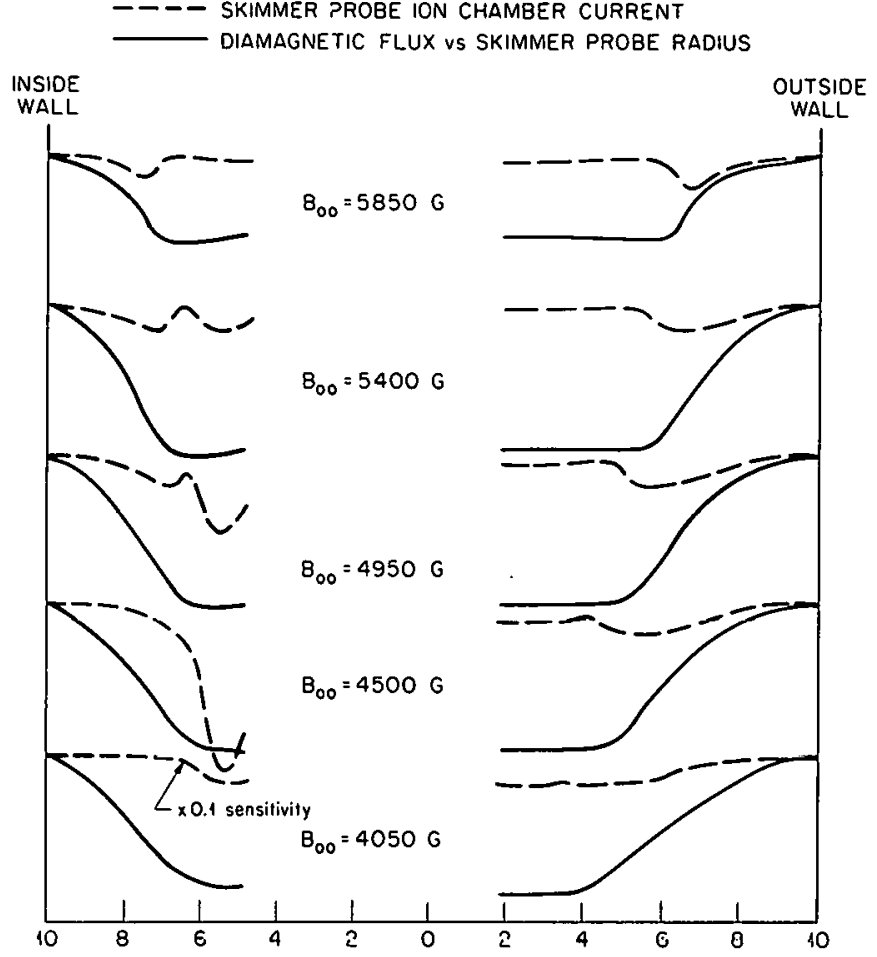

Fig. B.6. Measurements of location of annulus with various magnetic field strengths using a skimmer probe containing an ionization chamber to intercept energetic electruns. Sulfd 11nes: observed diamagnetic flux as a function of probe position. Dotted lines: jon chamber current. 
function of the probe location. Also shown is the observed diamagnetic flux measured in that sector. At $B_{00}=0.5 \mathrm{~T}$ (magnetic field strength at a coil pair midplane on axis), the presence of energetic electrons was measured inward to a radius of $\approx 12 \mathrm{~cm}$ when the probe was inserted from the outside wall. The diamagnetic flux half value point was $r \approx 13 \mathrm{~cm}$. The outer point where energetic electrons were intercepted was $r \approx 20 \mathrm{~cm}$. Detailed analysis of the shape of the pressure profile which produced these data is complicated by changes in equilibria produced by the probe itself, but a simple plot of the range of $\Delta \phi$ as a function of magnetic field is shown in Fig. B.7. Also shown is a curve of the points where the values of magnetic field in the midplane are at the second harmonic of electron cyclotron resonance for the applied $18-\mathrm{GHz}$ power. The experimental points indicate that hot electrons were found near these locations; the relatively narrow radial extent demonstrates the annular character of this component of the plasma.

The location of the toroidally confined plasma was determined from measurements of ion saturation current to a Langmuir probe (in a nonpowered toroidal sector). The current observed as a function of radial position is shown in Fig. B.8. For comparison, the calculated drift surfaces for passing particles (i.e., surfaces of constant $J=\oint p_{\|}$ds with $v=v_{\|}$in the midplane) are also shown. The probe (although water cooled) overheated when inserted too close to the axis so data were only available for $r \geq 10 \mathrm{~cm}$ on the outside of the torus. Clearly, the maximum observed saturation ion current lay well within the electron annuli and approximately in the vicinity of closed drift surfaces. Later measurements showed the existence of toroidally directed fast electrons within the region of closed drift surfaces when the magnetic field errors were not cancelled (as in these experiments) and this may account, in part, for the low currents seen at the center of the region with closed drifts. In any case, from these measurements it was seen that the toroidal plasma was spatially located inside the diamagnetic annuli and flowed stably about the torus.

\section{B.3 STABILITY OF THE ELECTRON ANNULUS}

The electron annulus plasma component in ËB̈ was observed to be stable when the ambient neutral density was sufficiently high. A curve typical of early measurements of the stable decay of the diamagnetism following interruption of the applied power is shown in Fig. B.9, in this case indicating a decay time of $\approx 0.25 \mathrm{sec}$. As the ambient neutral density was lowered, a point was reached in which fast spikes were noted in the diamangnețic sịanal, correspondinq to randomlv occurring instability of the electron annuli. These fluctuations occurred independently in the various mirror sections. A comprehensive experimental examination of the stability boundaries and conditions required for stability has not yet been undertaken, but the behavior described above is consistent with the requirements of appreciable cold plasma density for annulus stability ( $\left.n_{\text {cold }} / n_{\text {hot }} \geq 1\right)$. A significant fact is that stability of the annular plasma was observed even at low magnetic fields when the pressure profile peaked close to the toroidal axis (see Fig. B.7). Under these conditions, the flux lines through the annuius region did not intercept a wall. Thus, except for the outer edges of the annular plasma which could contain only a small fraction of the stored energy, "iine tying" to metal surfaces as a mechanism for stabilization must be ruled out. It is sufficient to remark here that no contradiction with theoretically predicted behavior was noted experimentally.

\section{B.1 MEASUREMENT OF PLASM PARAMETERS IN T MODE}

\section{B.4.T ELECTRON DENSITY}

The line density of electrons was measured using a 70-GHz microwave interferometer with the phase shift for null mixed output mechanically servoed to yield a precise analog output which could be 
ORNL-DWG 75-6396R

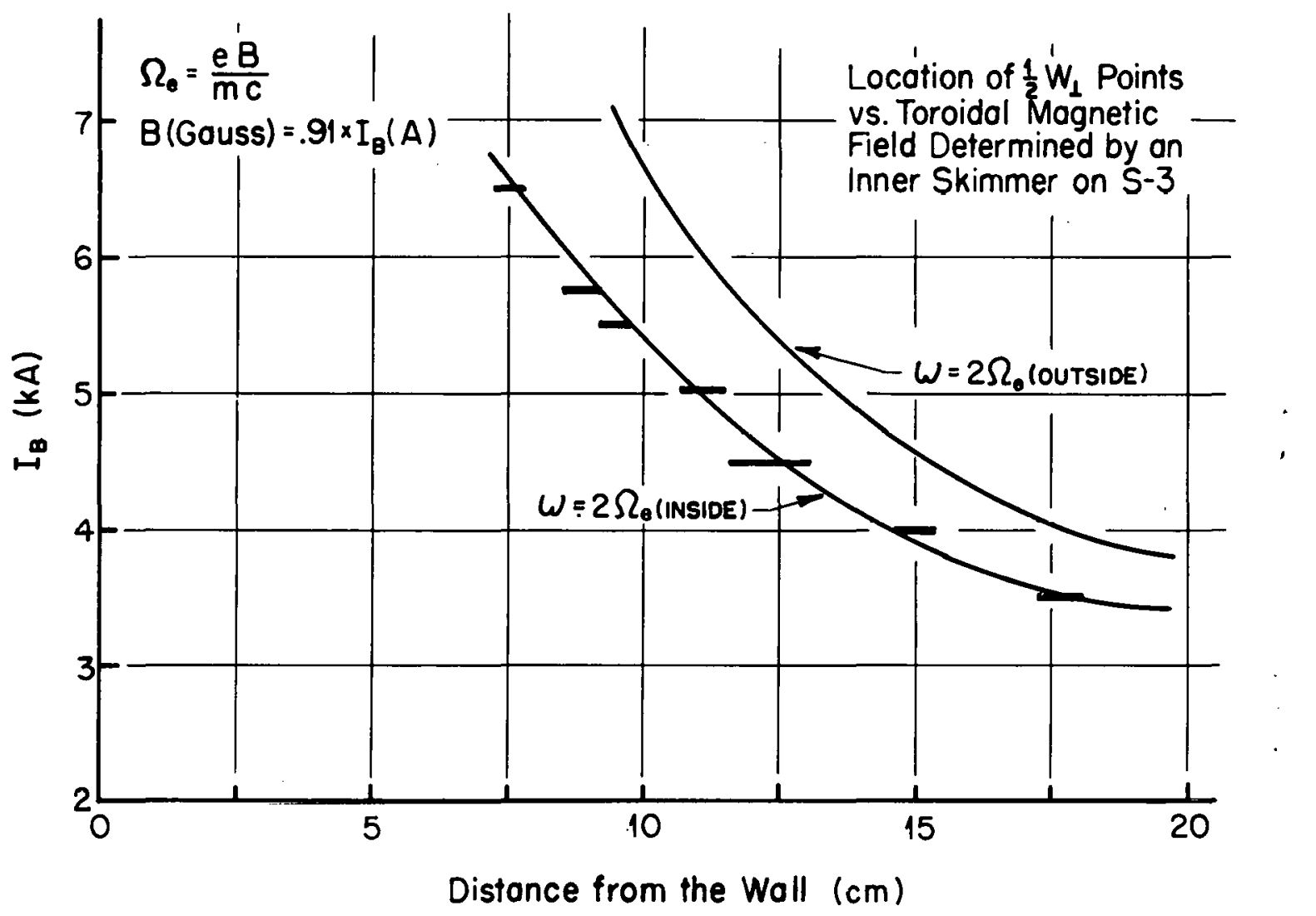

Fig. B.7. Location of electron annulus from data in Fig. B.3 as a function of magnetic field $\left(I_{B}=\right.$ magnet current). The solid curves indicate calculated location for $\omega=2 \Omega e$ for the applied frequency $(\omega=18 \mathrm{GHz})$. 
ORNL - DWG 74-10544

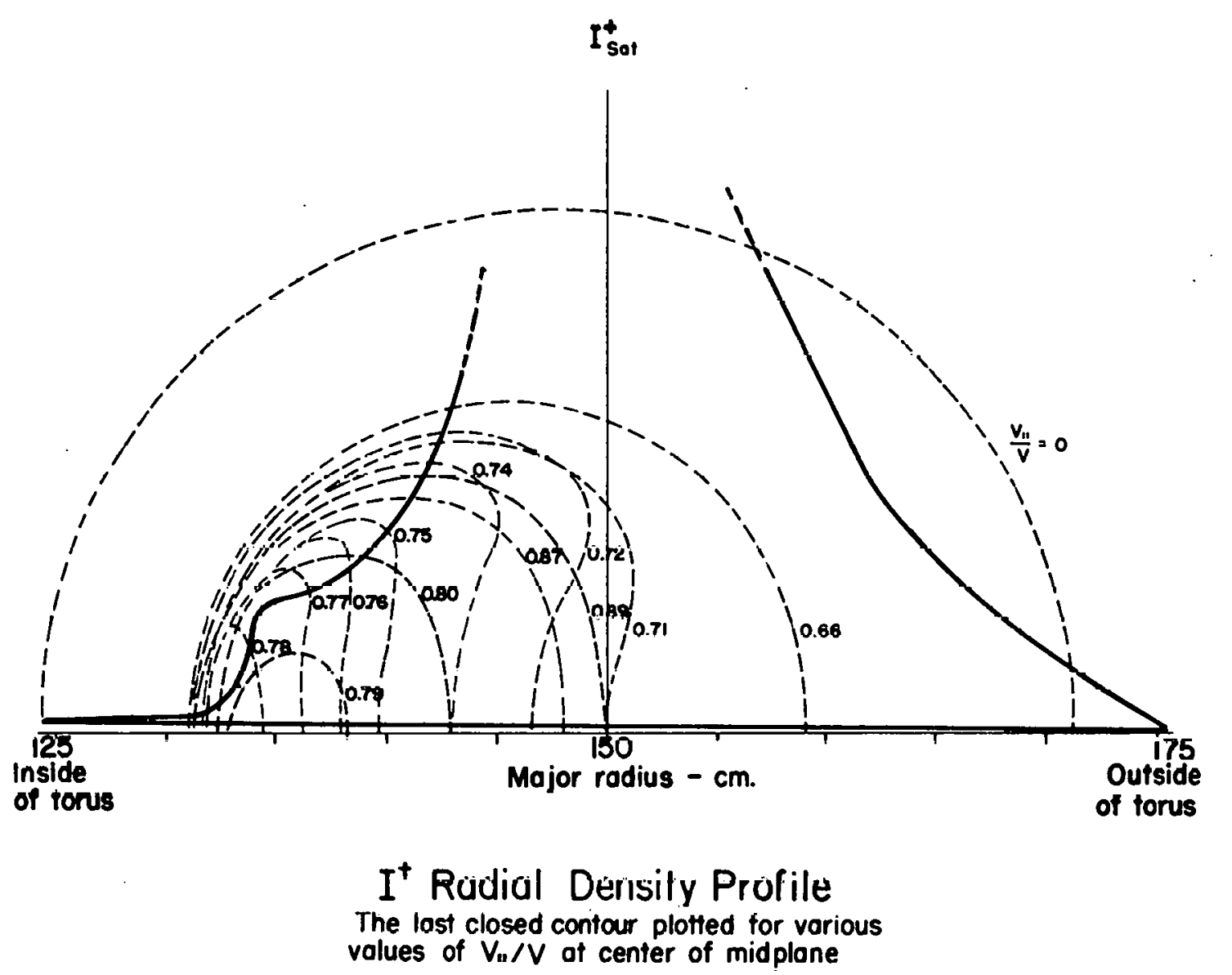

Fig. B.8. Saturation ion current measured with Langmuir probes as a function of radius. Dotted curves show closed drift surfaces for particles with various values of $v_{\| l} / v$ in the midpl ane. 


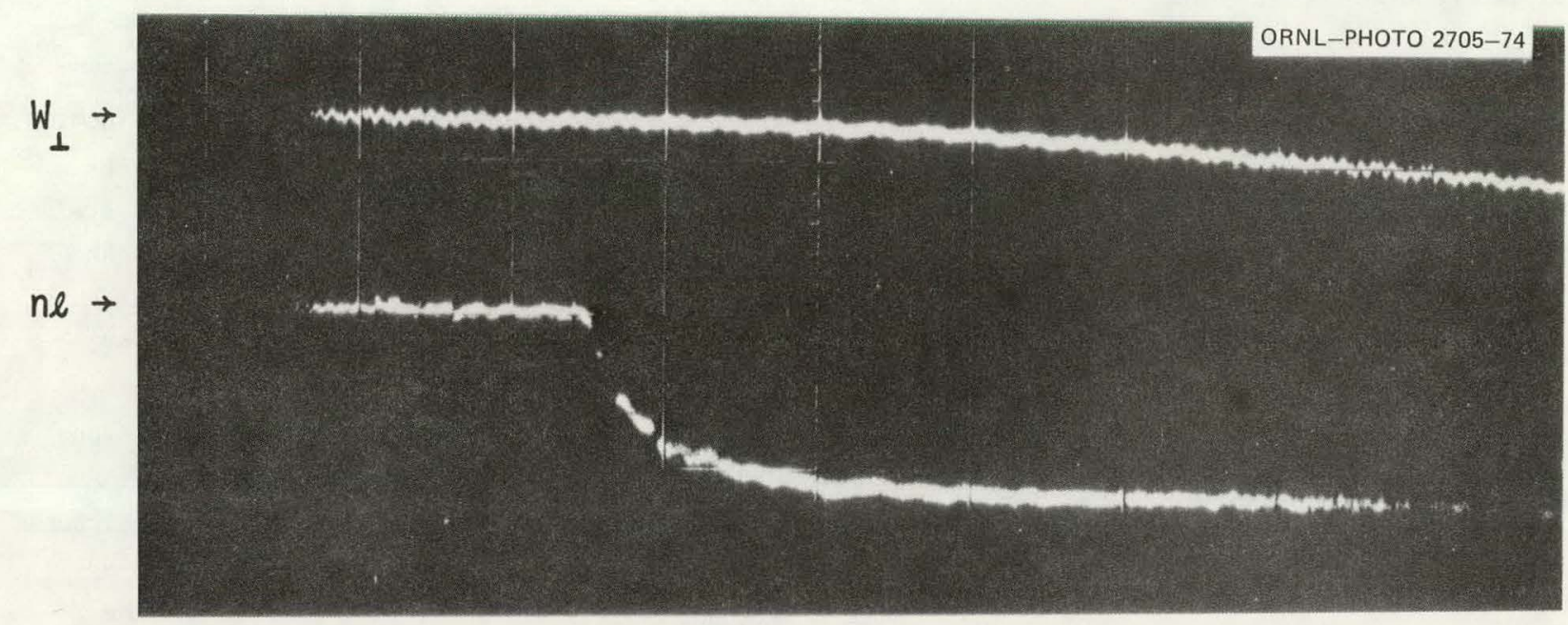

\section{$50 \mathrm{msec} / \mathrm{cm}$}

Fig. B.9. Tracing of decays of $W_{1}$ (measured with diamagnetic loop) and $n_{e} l$ (measured with interferometer) following turnoff of microwave power. Horizontal scale is $50 \mathrm{msec} / \mathrm{cm}$. 
continuously displayed. The plasma diameter was taken from the I angmuir probe data (Fig. B.8), which indicated $\ell \cong 20-30 \mathrm{~cm}$. From the observed phase shifts in T-mode,

$\mathrm{n \ell}=2-5 \times 10^{13} \mathrm{~cm}^{-2}$.

As described below, the density could also be deduced from bremsstrahlung measurements. These measurements yielded densities somewhat lower than the values quoted above, but since the temperature profile was certainly not flat and the enerqy range availahle for measurement was limited, this result is not surprising. In this discussion of the behavior of the toroidal plasma as a function of power or pressure, the relative density from bremsstrahlung observations normalized at the maximum values observed with both diagnostics has been used.

\section{B.4.2 ELECTRON TEMHERAIURE}

Two techniques were used to cstablish the electron temperature in the reginn of rlnsed $\mathrm{drift}$ surfaces during operation of EBT in the stable T-mode. An experiment was carried out to detect. Thomson scattered light at $90^{\circ}$ using a 1-1 repetitively pulsed ruby laser as a light source. The receiver optics and geometry are shown in Fig. B.10. Data were obtained with only $20 \mathrm{~kW}$ of heating power at $18 \mathrm{GHz}$. At the limited plasma density $\left(\mathrm{n}_{\mathrm{e}} \sim 10^{12} \mathrm{~cm}^{-3}\right)$ and in the presence of a large plasma light background signal, it was difficult to obtain data over a broad range of operations. However, the experiment was valuable in establishing, unambiguously, a warm electron component with $T_{e} \sim 150 \mathrm{eV}$.

The principal diagnostic tool used for electron temperature determinations was a "windowless" lithium drifted, silicon barrier [ $\mathrm{Si}(\mathrm{Li})]$ detector which was collimated to observe the bremsstrahlung radiation from the toroidal electron distribution. The detector geometry is shown in Fig. B.11. A typical count rate vs energy spectrum (Fig. B.12) illustrates the complications which had to be circumvented to establish the electron temperature. Since the anticipated temperatures were helow 500 eV, it was necessary to subtract the continuum produred hy the energetic clectrons in the annulus and lie aluminum $k_{\alpha} x$-ray 1 ine at $1.49 \mathrm{keV}$. The exponential decrease in photon intensity with energy and the modest electron temperature constrained the critical range of useful information to between 500 and $1200 \mathrm{cV}$. In this range, the efficiency of the detector was strongly influenred by material which condensed on the liquid nitrogen cooled detector face. Efficiency calibration of the system in this range was carried out in place by operating EBT so that the energetic annulus was produced but no appreciable toroidal plasma could be present. This was accomplished by energizing only those magnetic. field coils in the mirror section viewed by the detector. The known continuum shape at low photon energies produced by the energetic electrons constituted an ideal radiation standard. The differences between observed count rates and those predicted from the nhservatinns at higher energy resultcd in efficiency corrections which could be applied to the data when the facility was subsequently operated in $\mathrm{T}$-mode. A typical reduced spectrum with $T_{e}=400 \mathrm{eV}$ is shown in Fig. B.13. The furmuld fur intensity $[I(E)]$ is

$I(E) d E=3.73 \times 10^{-21} n_{e}^{2} l T^{-1 / 2} e^{-E / T} e d E(k e V / s e c)$,

where $n_{e}$ is in (centimeters)-3, $\ell$ is in centimeters, and $E$ and $T_{e}$ are in kilnelertron-volts; $\ell$ is the length of plasma and the constant includes the geometric corrections for solid angle viewed. Both the density and the electron temperature can be derived from the data. From observations with various operating conditions, the range of electron temperatures in EBT in T-mode lay between 150 and $600 \mathrm{eV}$. 
ORNL-DWG 75-18281

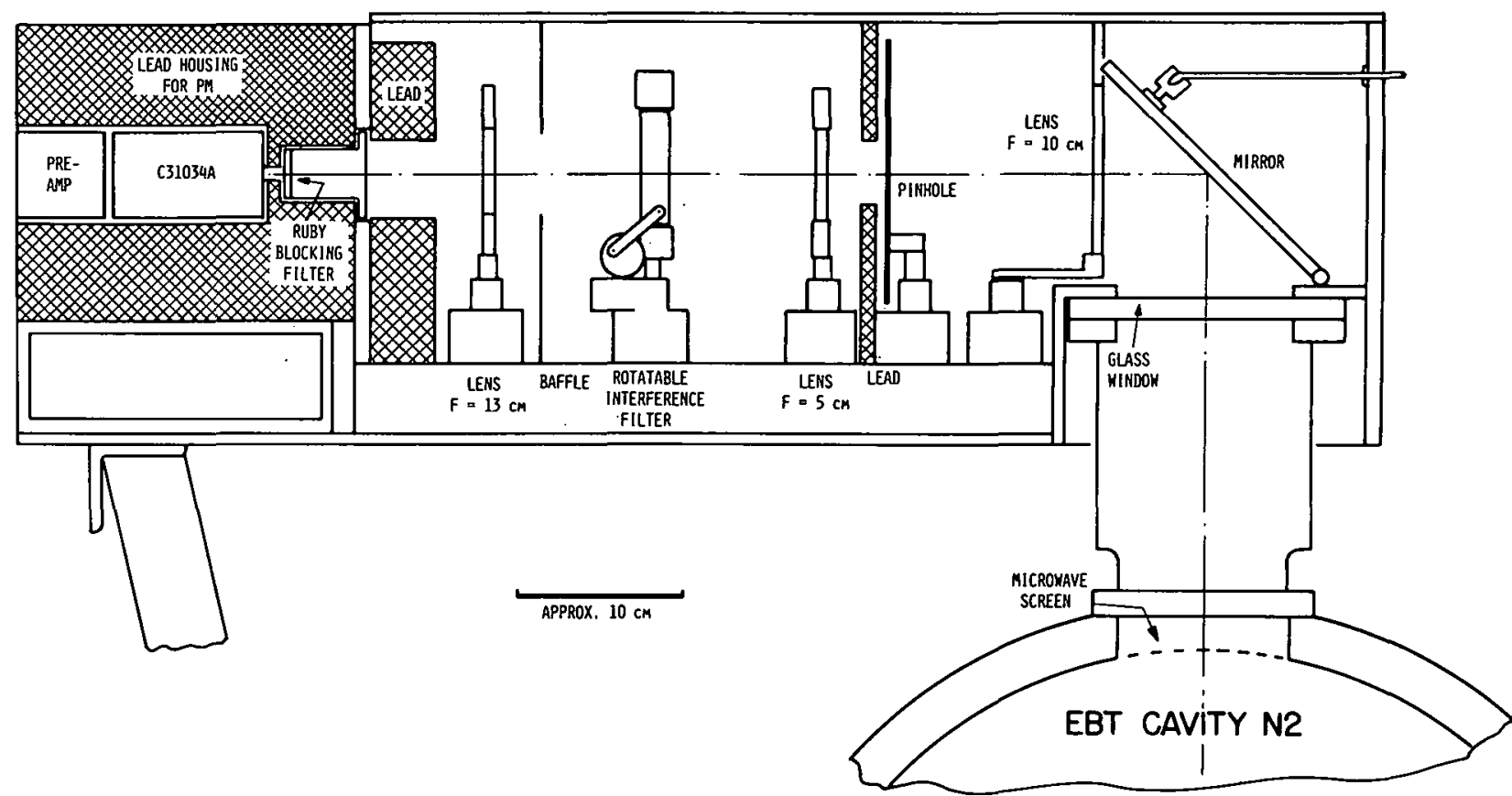

Fig. B.10. Receiver optics for Thomson scattering measurement of ruby laser light at $90^{\circ}$.

ORNL/DWG/FED -77352

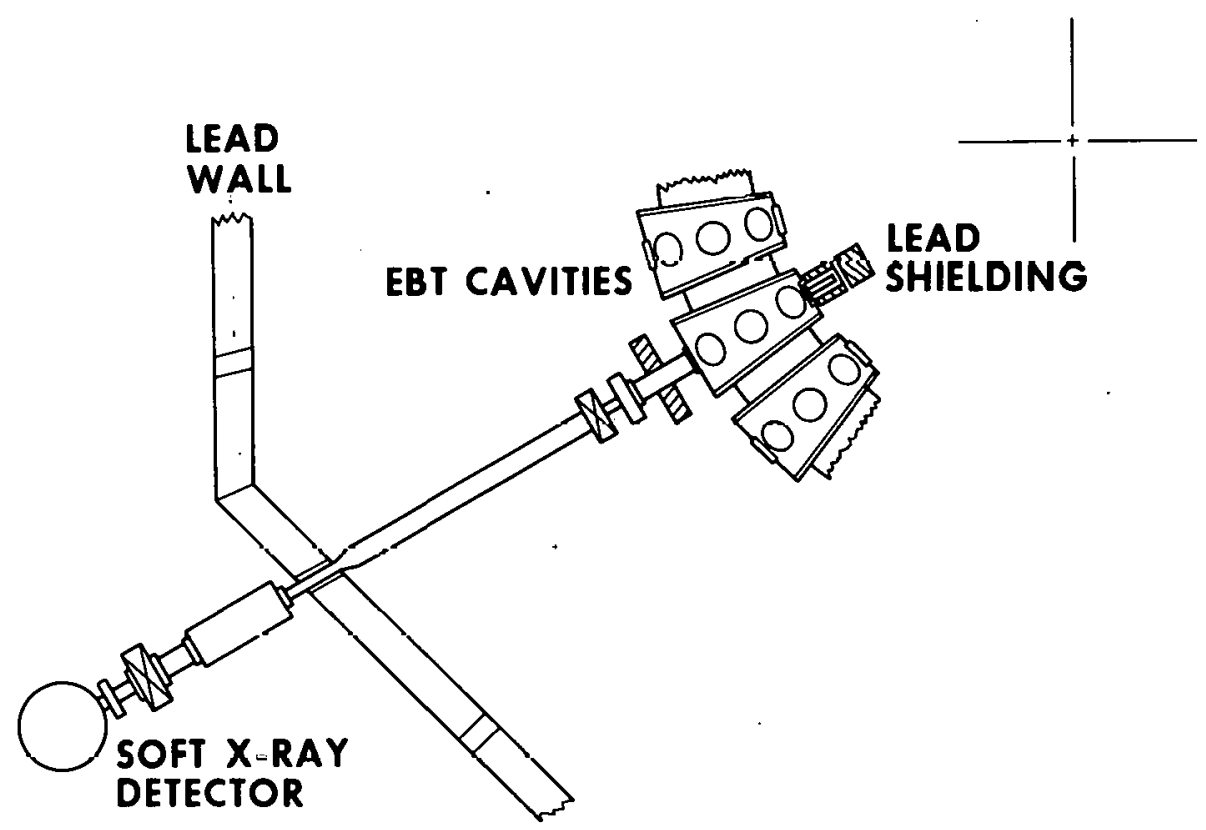

Fig. B.11. Geometry used with $\mathrm{Si}(\mathrm{L} i)$ detector for viewing bremsstrahlung in $\mathrm{EBT}-\mathrm{I}$. 


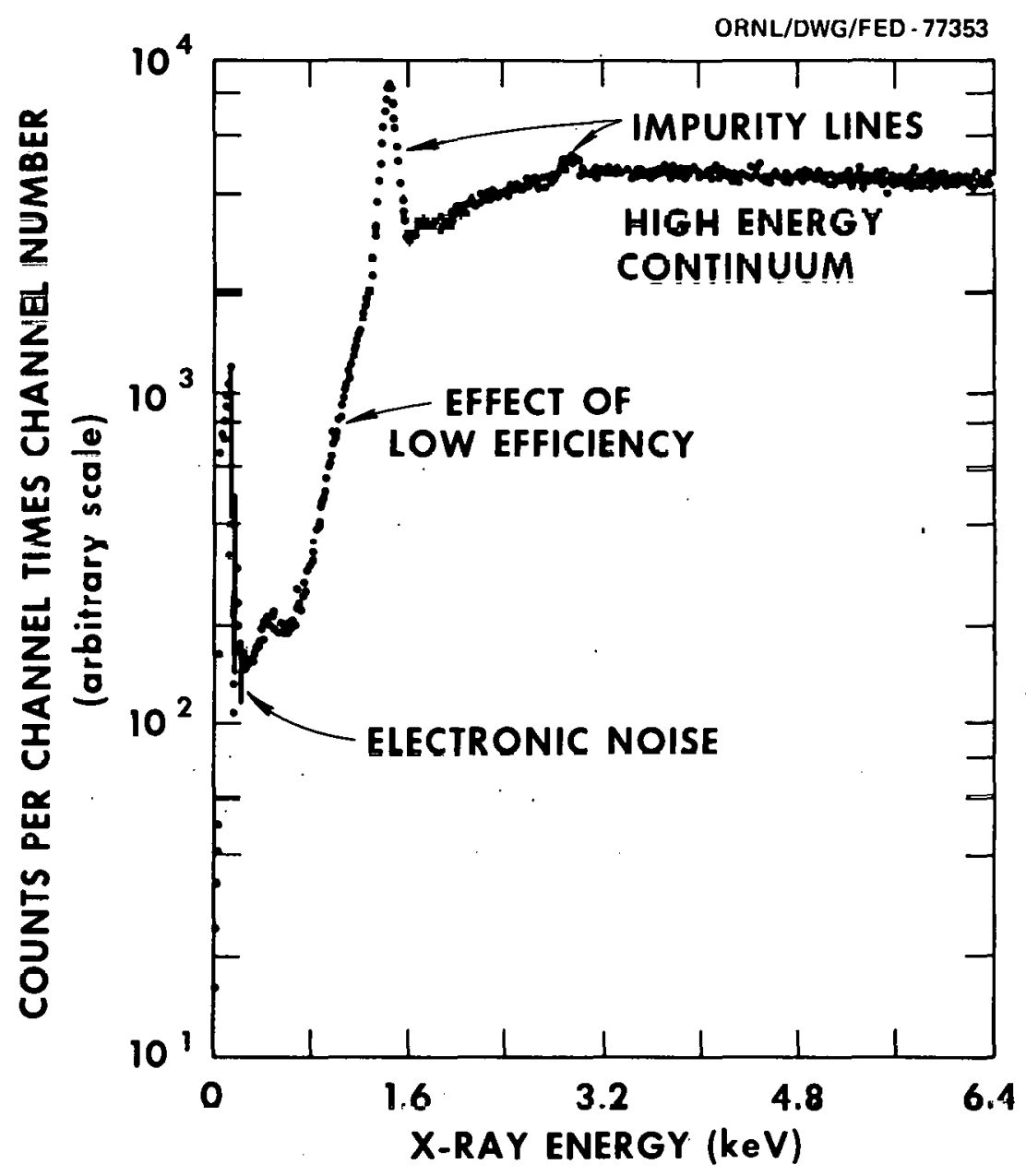

Fig. B.12. Count rate spectrum obtained with $S i(L i)$ detector collimazed to view line through equatorial plane. 


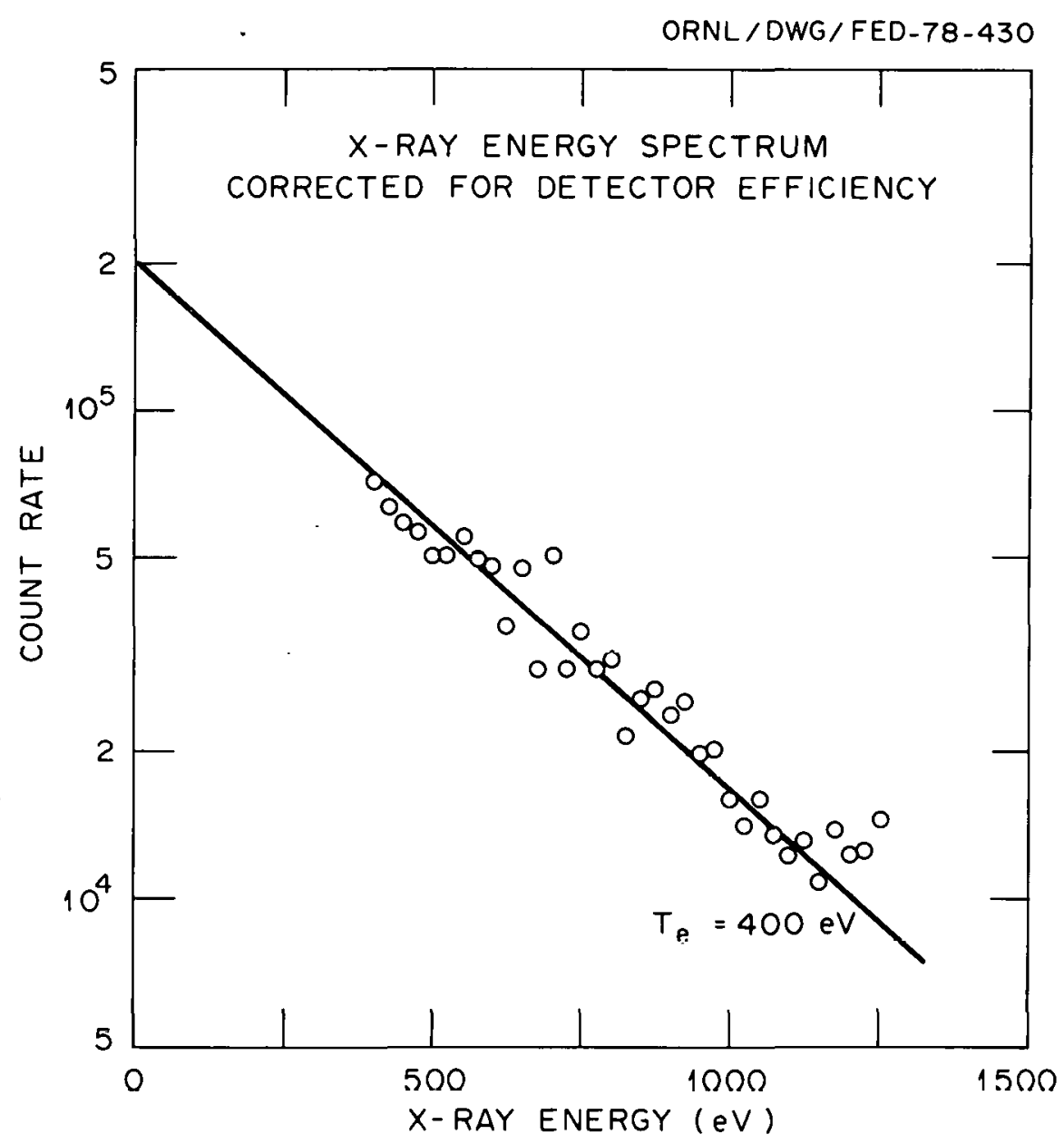

Fig. B.13. Count rate distribution obtained with Si(Li) detector after correction for efficiency. 


\section{B.4.3 ION TEMPERATURE}

The ion temperature in EBT-I was determined by analysis of the energy spectrum of the energetic $H^{0}$ or $D^{0}$ atoms escaping from the plasma as a result of charge exchange collisions between plasma ions and background neutral gas. Four different charge exchange neutral spectrometers were used on EBT-I, each one sampling neutral particles escaping perpendicular to the toroidal magnetic field in the midplane of a toroidal bump. Energy analysis on all the spectrometers was accomplished by converting the neutral

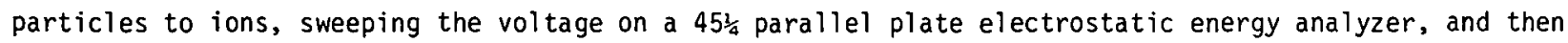
detecting the ions with a channel electron multiplier. Three of the spectrometers employed a differentially pumped nitrogen stripping cell to convert the neutral particles to positive ions; the fourth used a cesium charge exchange cell ${ }^{3}$ to convert the escaping neutral particles to negative ions. One spectrometer employed a velocity filter for mass analysis of the esraping nelltrals, and one spectrometer was a 4-beam device sampling four different chords of the plasma.

The typical geometry used in the charge exchange measurements is shown in Fig. B.14, with the neutral particle conversion cell in this case being a cesium cell. Calibration of the entire system was carried out with a low energy neutral particle beam whose intensity was calibrated against an ion beam at the same energy using a secondary emission detector as the primary detector. The secondary emission coefficient for hydrogen ions and neutrals was compared in a still unpublished experiment by Barnett, and the variation of this parameter with energy was included in a recent reevaluation of the data. The recalculated efficiency for the system, using the nitrogen and cesium cells, is shown in Fig. R. 15 .

Typical spectra of charge exchange neutrals for the same plasma measured with the cesium cell and nitrogen stripping cell, corrected for efficiency, are shown in Fig. B.16. Analysis of the data using the cesium cell, which has larger apertures and much higher efficiency, particularly at the lower energies, indicates a higher average energy and a lower $\overline{n_{j} n_{0}^{l}}$ than were obtained from the nitrogen cell data. In both cases, the spectra were non-Maxwellian, as one would anticipate from the nonuniform neutral density and ion temperature profiles. The anticipated cesium cell advantages as a consequence of the higher efficiencies at lower energy have not yet been fully exploited because of a disturbing residual background count rate produced by photoelectrons. Further, the cell efficiency may be somewhat questionable at the lower energies because the calibration beam diameter was considerably smaller than the aperture size, leading to a possible overestimate of cell efficiency because of uncompensated scattering in the cell for uncentered particles. These uncertainties are expected to be resolved in tuture experiments.

Ion temperature measurements with both analyzers were made over a range of operating conditions in EBT-I. Data were taken over the range from C-mode through many combinations of pressure and power in $T$-mode to the $T-M$ mode transition. Central ion temperatures in T-mode ranged from $\approx 30 \mathrm{eV}$ near the transition between $C$ - and $T$-modes to $\approx 150 \mathrm{eV}$ at the lowest pressure.

The value of $\overline{n_{j} n_{0}^{l}}$ which is derived from the spectrum is of significance since it establishes whether the bulk of the ion population is observed (e.g., as contrasted with a possible ion runaway population). The value obtained from analysis of the nitrogen stripping cell data, $\overline{n_{j} n_{0} l} \sim 1.2 \times$ $10^{22} \mathrm{~cm}^{-5}$, is somewhat lower than spectroscopic estimates of $n_{0} n_{e}$ at the plasma center if one assumes that the hottest ions are confined nearer the axis with $\ell \cong 10 \mathrm{~cm}$. However, the nonuniform temperature profile introduces an additional uncertainty in the analysis which can only be removed by spatially resolved measurements of the neutral flux.

\section{B.4.4 SPACE POTENTIAL}

Confinement of plasma in the toroidal EBT geometry differs fundamentally from that in tokamaks because of the absence of a poloidal magnetic field component. In EBT the unequal radial fluxes of 


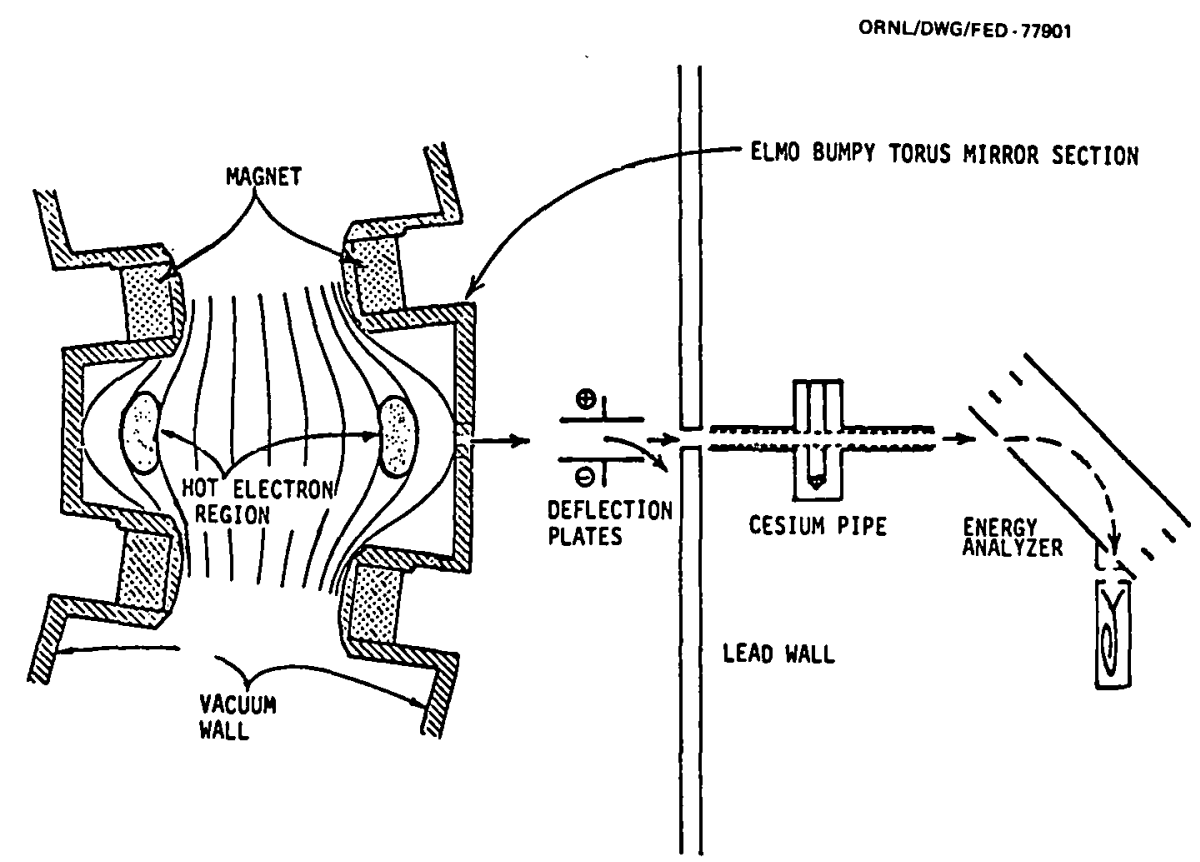

ENERGY ANALYZER SYSTEM ON ELMO BUMPY TORUS

Fig. B.14. Schematic arrangement of system used to detert. charge. exchange neutral particles from EBT-I. The distance from the center of the toroidal sector to the cesium cell reionizer is approximately $2.5 \mathrm{~m}$. 


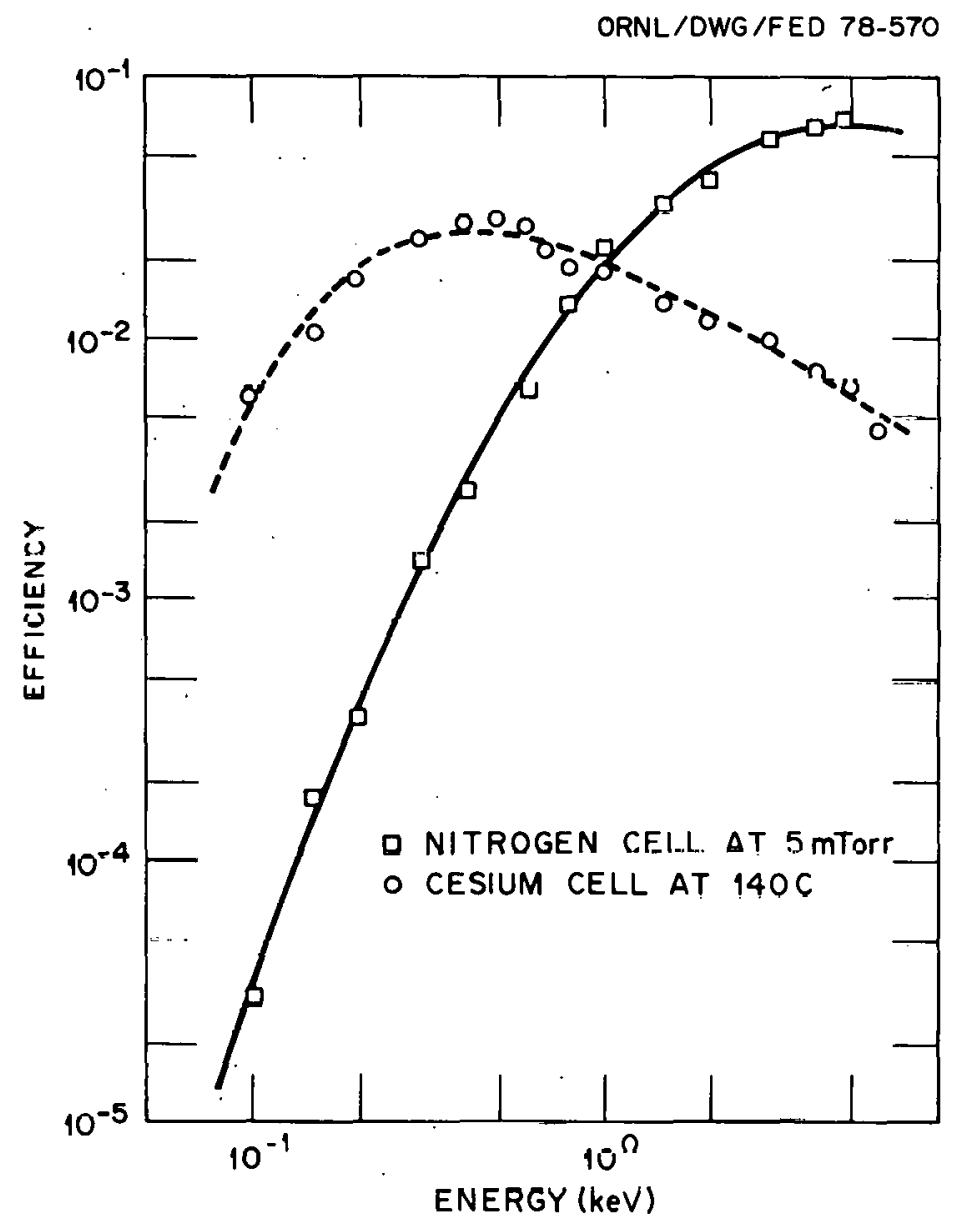

Fig. B.15. Efficiency of nitrogen stripping cell and cesium heat pipe charge exchange cell as a function of incident neutral particle energy in geometry used for ion temperature masurements. 

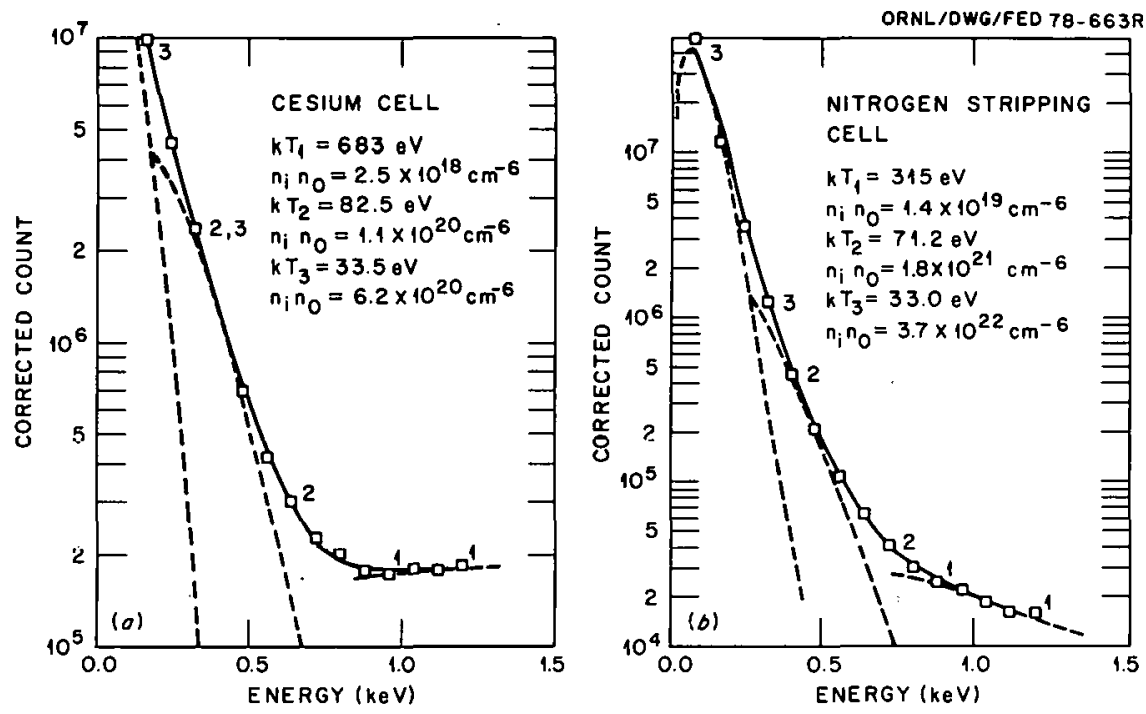

Fig. B.16. Typical count rate spectrum of neutral particle energy obtained with (a) the nitrogen and (b) the cesium spectrometers under T-mode conditions (microwave power: $40 \mathrm{~kW}$ at $18 . \mathrm{GHz}, 8 \mathrm{~kW}$ at $10.6 \mathrm{GHz}$; midplane magnetic field, $5 \mathrm{kG}$; neutral pressure, $5.1 \times 10^{-6}$ torr). The ordinate is the raw count divided by spectrometer efficiency. The curves are fit with three Maxwellian components. The solid curve is the sum of the three component fits. 
ions and electrons generate a self-consistent radial electric field which controls the ambipolar diffusion rate, whereas in tokamaks the unequal radial particle fluxes drive a toroidal current which performs the same function. Therefore, it was of fundamental importance to the understanding of plasma confinement in EBT that the space potential be measured as a function of position.

R. L. Hickok and colleagues ${ }^{4}$ developed a technique for measuring space potential using a heavy ion beam probe. The energy of singly ionized heavy ions entering a plasma with space potential $\phi_{S}$ is reduced by $e_{S}$. Doubly ionized ions produced by collisions with electrons in the plasma emerge with energy $2 \mathrm{e} \phi_{S}$ greater. The difference in energy between the injected beam energy and secondaries is e $\phi_{S}$. The ion orbits could be calculated precisely and those secondary ions which enter the detector traced back to a locus of points in the plasma. The intersection of these points with the beam path established the point in space where the secondary ionization took place (Fig. B.17). By varying the beam energy and incident angle, the entire two-dimensional space in the midplane could be traced out, producing a map of the electric field in the plasma.

The rather considerable adaptation of this technique to the stcady state mode and plasma parametcr regime of $E^{\prime} l^{\circ}$ is depicted in the overall ion beam system (Fig. B.18): The ion beam (generally $\mathrm{Rb}^{+}$, a though $\mathrm{K}^{+}$and $\mathrm{Na}^{+}$were sometimes used) was generated and accelerated by a gun structure mounted above the midplane of a cavity in EBT. The beam passed through the sweep chamber and could be swept across the plasma (in the plane of the figure) or along the plasma (out of the plane of the figure). To minimize noise problems, the beam was usually chopped by driving the beam out of the plane with a high voltage pulse on the chop plates, and synchronous detection was used.

In practice, the energy difference between singly and doubly ionized particles was measured using a parallel plate electrostatic energy analyzer. Small changes in energy which occur due to secondary ionization at positions where $e_{s} \neq 0$ result in a deflection of the particles in the analyzer from their initially centered position; the voltage change on the top plate required to recenter them was proportional to this energy difference.

Calibration of the system was provided by nulling the acceleration voltage against the voltage on the analyzer required to center the primary beam ions directed onto the detector system and then using precision resistors to divide the analyzer voltage by precisely a factor of two for measuring potentials using $Z=2^{+}$lons; similar precision resistor ratios were used for nigher charge states. Closed loop feedback of the analyzer voltage tracked the beam for changes of the accelerator gun voltage using narrow band phase-sensitive detection at the beam chopping frequency to maximize the signal-to-noise ratio and accuracy.

The result of analysis of a typical scan with variable beam energy and incident angle with the plasma in the stable T-mode is shown as a potential map in Fig. B.19. Under optimum confinement conditions, the ambipolar electric field was nearly axisymetric, pointed radially inward, and of sufficient magnitude to strongly influence the poloidal drifts. The symmetry shown in Fig. B.19 was critically dependent on error field global nulling (see Sect. B.4.7) and T-mode conditions. For example, in C-mode, the scans were qualitatively different (Fig. B.20), the potentials were smaller, the electric field was reversed, and the symmetry was significantly reduced.

\section{B.4.5 IMPURITIES}

Radiation in the visible and vacuum uv was measured in order to determine the density of impurities and neutral hydrogen and to establish their spatial distributions. A 2.2-m Rowland circle grazing incidence spectrometer was used to measure resonance line radiation down to $\leqslant 50 \AA$. A $0.125-m$ Ebert spectrometer was used in the uv $(1200 \AA<\lambda<2300 \AA)$ with two scanning mirrors (Fig. B.21) to study the principal line radiation from carbon and aluminum as a function of spatial position. Analysis of 


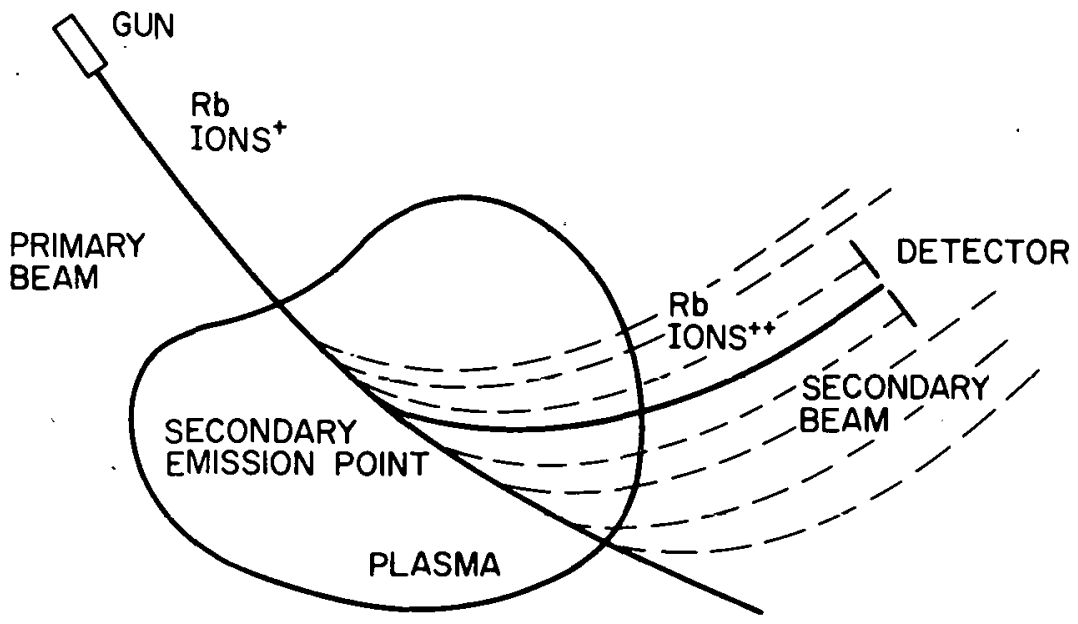

Fig. 8.17. Schematic drawing showing path of injected $\mathrm{Rb}^{+}$ions and orbits of secondarily ionized $\mathrm{Rb}^{++}$ions from various points along beam path. 


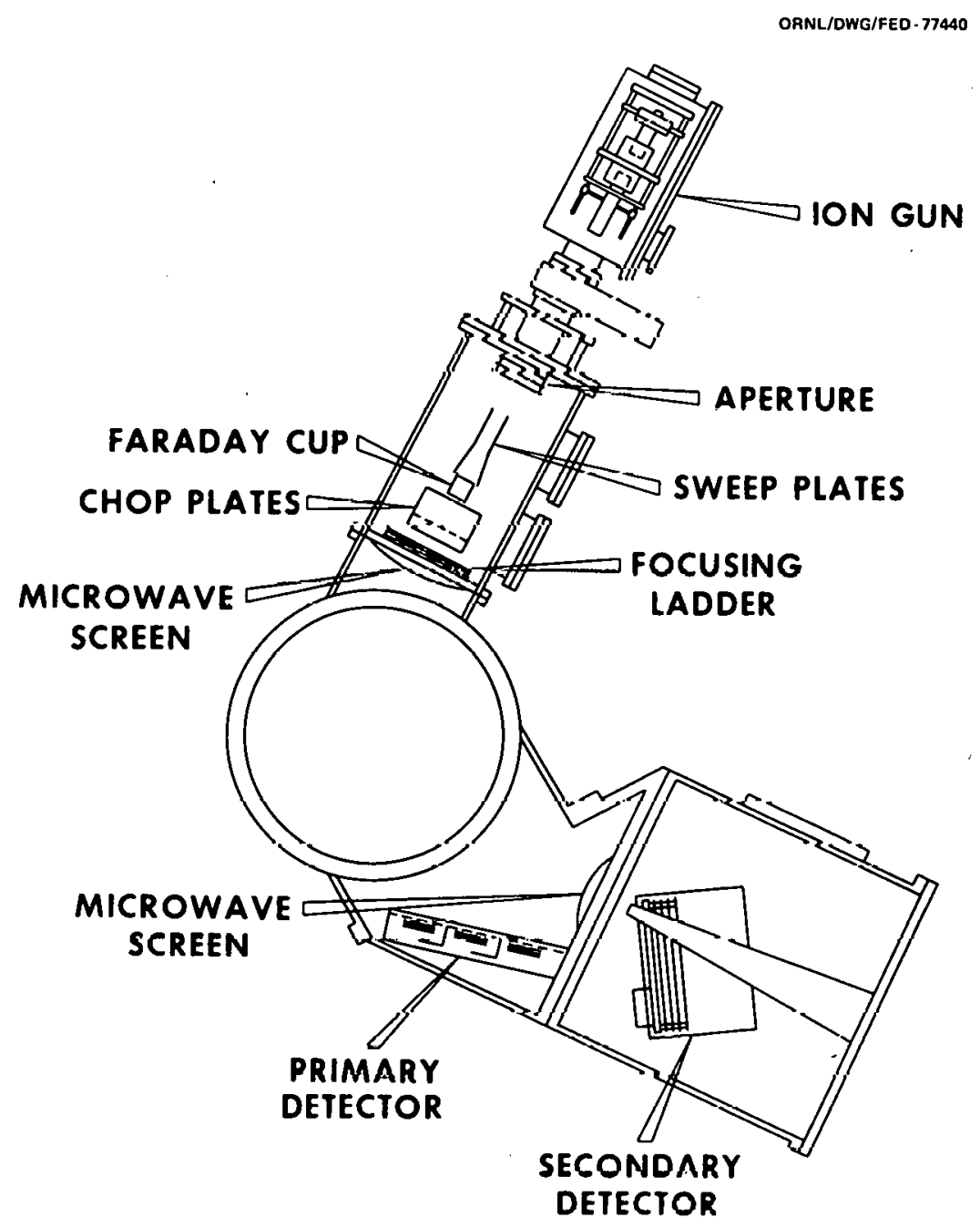

Fig. B.18, General layout of heavy ion bcam probc system used in EBT-I. Secondary detector is a graded field, parallel plate energy analyzer. 

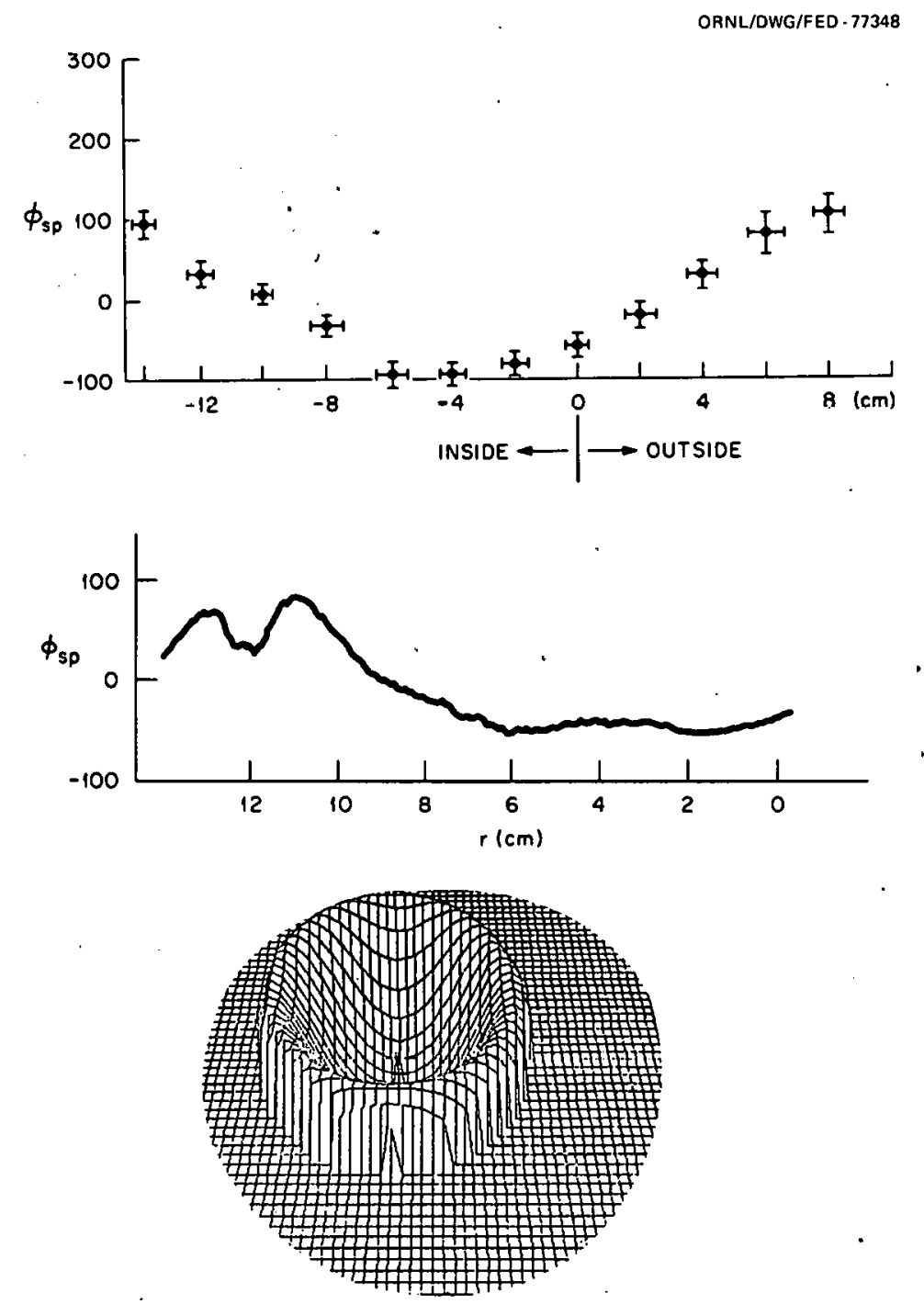

Fig. B.19. Space potential measured in EBT-I as a function of position in the midplane (T-mode conditions). The computer generated perspective view includes data taken at various energies and angles of beam incidence and then "spline fits" the data to present the smoothed display. No data are taken outside the points where the maximum potentials are found. 

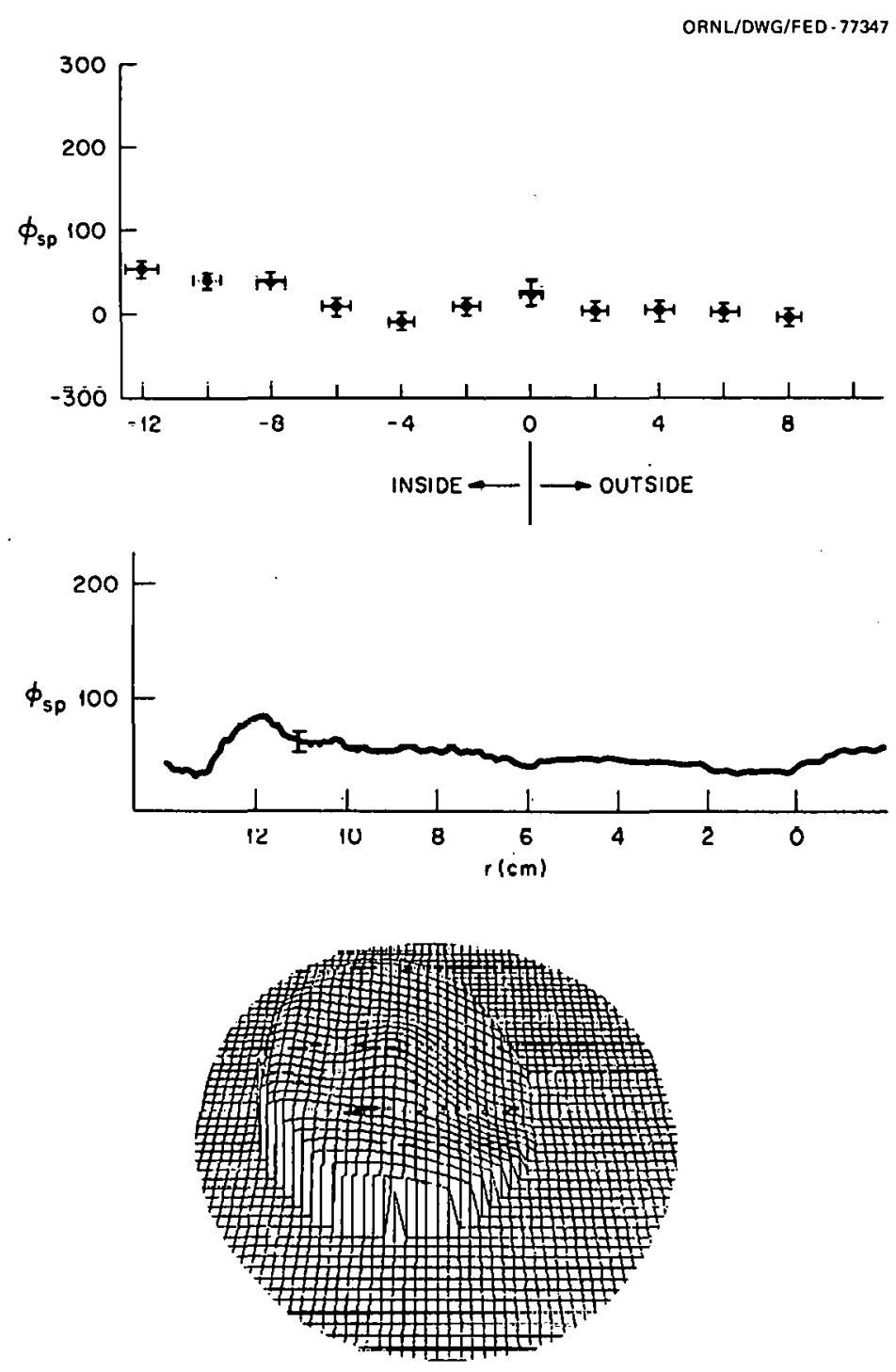

Fig: B.20. Spoce potentiol mensulped in EBT=I as a function of position in midplane ( $\mathrm{C}$-mode conditions). 


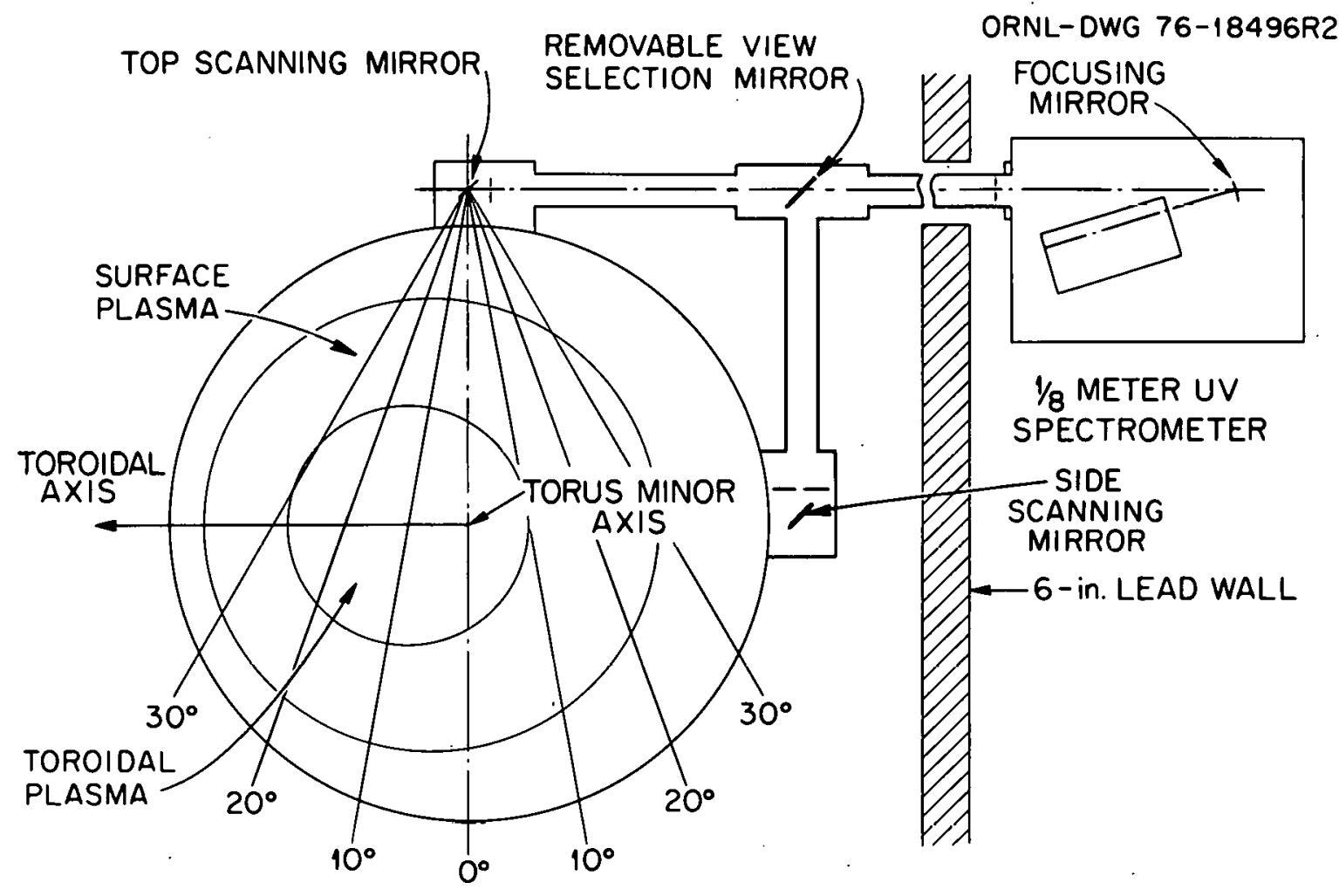

Geometry for Optical Scanning.

Fig. B.21. Geometry used for spatial scanning of uv light from plasma in midplane of EBT-I. Distance along 1 ight path to spectrometer focusing mirror is $22.75 \mathrm{~m}$. 
these data was carried out using an iterative algebraic reconstruction technique (ART), similar to methods developed for medical tomography, which permitted reconstruction of the light emission distribution even for asymmetric cases. 5 Finally, a $0.5-\mathrm{m}$ Ebert spectrometer was used in the visible region to continue the aluminum and carbon studies and to look at the ratio of line intensities from He 0 to measure the electron temperature in those regions in which this technique was relevant.

To determine the impurity densities from the observed light intensities, the electron density and temperature spatial distributions must be known. As established from the Langmuir probe measurements, besides the annulus, the EBT plasma consisted of two spatially separated electron distributions. These components differed in both energy and density because of their entirely different confinement properties. The toroidal plasma; near the axis of the device, was confined on closed drift surfaces, and its confinement time was therefore controlled by a radial diffusive transport rate out to flux lines which. ultimately intercept the vacuum walls. In contrast, the plasma which lay outside the radius of the annulus was confined on drift surfaces containing flux lines which intersected the vacuum walls. Thus, the confinement time of these plasma particles was controlled by their polnidal presession ratep onto these open flux lines and the diffusion time in velocity space which permitted them to attain sufficient $v_{\|} / v$ to escape the mirror trap.

The measurements showed that in the stable T-mode, the surface plasma had an electron temperature of $\approx 30 \mathrm{eV}$ and an average density of $\approx 3 \times 10^{11} \mathrm{~cm}^{-3}$. Impurity ions were also found in the toroidal plasma, although for all species the density was observed to be lower than that seen in the surface plasma.

The densities observed at various radii in the surface plasma, multiplied by the electron density, are 1 isted in Table 8.2 for a typical stable T-mode operation. The rate equation at equilibrium for a particular charge state, $j$, is

$\frac{d n_{j}}{d t}=0=n_{j-1} n_{\theta}\left\langle\sigma v>{ }_{j-1}^{j}+n_{j+1} n_{e}\left\langle\sigma v>j_{j+1}^{j}-n_{j} n_{e}\left\langle\sigma v>\underset{j}{j+1}-n_{j} n_{c}\left\langle\sigma v>{ }_{j}^{j-1}-\frac{n_{j}}{{ }^{r} j}\right.\right.\right.\right.$,

where $\sigma v_{j}^{j+1}$ is the ionization rate coefficient from state $j$ to $j+1, \sigma v_{j}^{j-1}$ is the recombination rate coefficlent, and $\tau_{j}$ is the ion continement time in state $j$. Neglecting recombination for the densities and temperatures in EBT-I, the value of $n_{e} \tau_{j}$ could be calculated from measured values of the ratio $n_{e} n_{j} / n_{e} n_{j-1}$. The values of $n_{e} \tau_{j}$ obtained are also listed in Table B.2. Table B.3 lists the impurity ion densities. Table B.2. Product of electron density with carbon ion density $\left(n_{e} n_{j}\right)$
and ion lifetime $\left(n_{e^{\tau}}\right)$ in the surface plasma region of EBT

\begin{tabular}{llll}
\hline Species & $\begin{array}{c}\text { Radius } \\
(\mathrm{cm})\end{array}$ & $n_{e^{n}}\left(\mathrm{~cm}^{-b}\right)$ & $n_{e^{\tau} j}\left(\mathrm{~cm}^{-3} \mathrm{sec}\right)$ \\
\hline C II & 10 & $1.5 \times 10^{20}$ & \\
& 15 & $0.1 \times 10^{20}$ & $1.2 \times 10^{8}$ \\
C III & 10 & $1.6 \times 10^{20}$ & $1.4 \times 10^{8}$ \\
& 15 & $1.4 \times 10^{20}$ & $1.0 \times 10^{8}$ \\
C IV & 10 & $3.6 \times 10^{19}$ & $0.8 \times 10^{7}$ \\
\hline
\end{tabular}


Table B.3. Impurity ion densities in the region of the toroidally confined plasma $\left(n_{e}=1.5 \times 10^{12} \mathrm{~cm}^{-3}\right)$

\begin{tabular}{cc}
\hline Ion charge state & Density $\left(\mathrm{cm}^{-3}\right)$ at $r=0$ \\
\hline C I I & $1.0 \times 10^{8}$ \\
C III & $1.2 \times 10^{8}$ \\
C IV & $3.0 \times 10^{7}$ \\
C V & $<1.5 \times 10^{7}$ \\
AI II & $1.6 \times 10^{8}$ \\
AI III & $4.6 \times 10^{8}$ \\
\hline
\end{tabular}

These figures imply confinement times of considerably less than $1 \mathrm{msec}$ for the impurity ions in the surface plasma. In effect, the surface plasma acted as a divertor to ionize incoming neutral impurities and direct them to the walls before they diffused into the toroidal plasma where they could affect confinement. The divertor effect was enhanced by the presence of the large positive potential barrier observed at the position of the electron annulus (Fig. B.19), which had to be overcome by impurity ion heating if impurities were to reach the toroidal plasma. In the short lifetime of the impurity ions, such heating could not be accounted for by classical processes.

To illustrate the spatial reconstruction technique, the neutral aluminum emissivity is shown in Fig. B.22. The deep "hole" in the profile illustrates the effectiveness of the surface plasma in ionizing incoming neutral atoms. Figure B.23 shows the reconstructed light emission profile from singly ionized aluminum. The peak at the center could not be accounted for from the neutral particle source in Fig. B.22. The explanation for the AI II peak is that sputtered aluminum atoms from the walls in the mirror throats, which were not seen in the midplane, penetrated the toroidal plasma as unexcited neutrals and were then ionized. Excitation of these ions followed as they streamed along flux lines to the midplane and the subsequent deexcitation light was seen by the spectrometer.

\section{B.4.6 NEUTRAL HYDROGEN}

Neutral hydrogen played an important role in the plasma equilibrium since it was the source of particles which sustained the plasma against losses to the walls. Spectroscopic measurements of light helped to determine the neutral density distribution of hydrogen and the average hydrogen atom velocity. The latter measurement was particularly significant since the mean free path for neutral penetration, $\lambda$, depended on the atomic velocity as

$\lambda=\frac{v_{u}}{n_{e}\left(\langle\sigma v\rangle_{i o n}+\langle\sigma v\rangle_{c x}\right)}$,

with $v_{0}$ the atom velocity, $\langle\sigma v\rangle_{i o n}$ the ionization rate coefficient, and $\langle\sigma v\rangle_{c x}$ the charge exchange rate coefficient averaged over the electron velocity distribution. Particle losses out of the plasma center had to be balanced by ionization, so

$\frac{n_{e}}{\tau}=n_{e} n_{0}\langle\sigma v\rangle{ }_{i o n}$.

As pointed out in Sect. B.3., for stability of the annulus, it was necessary that the cold plasma density (and therefore neutral density for its support) in the surface plasma must be relatively high 

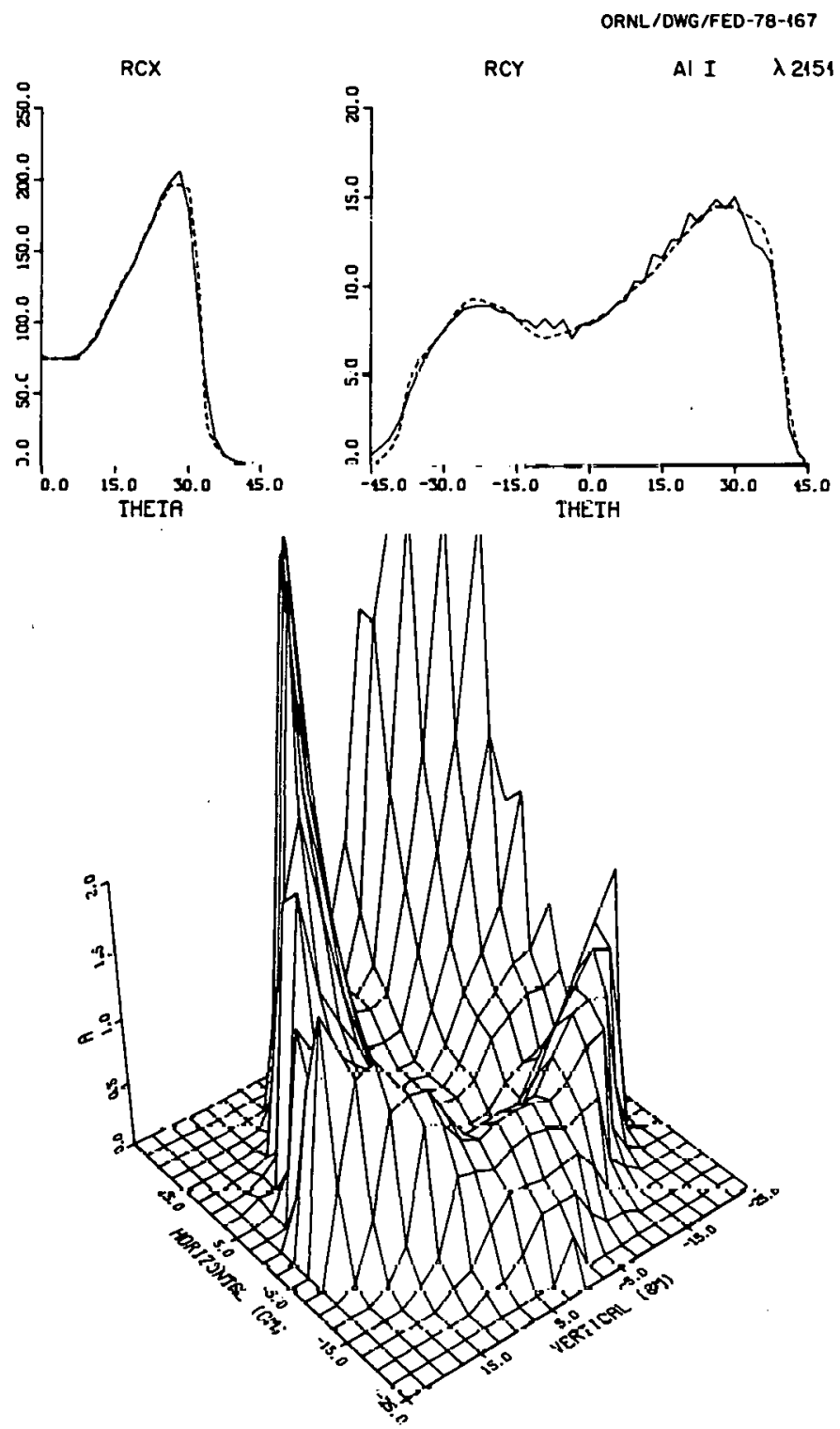

rig. B.22. Light intensities from Al I at $\lambda=2151$ \& as a function of anglo of view ( $\theta$ ) from top (RCY) and side (RCX) ports. Lower drawing is reconstructed emission distribution in midplane calculated using iterative algebraic reconstruction technique. 

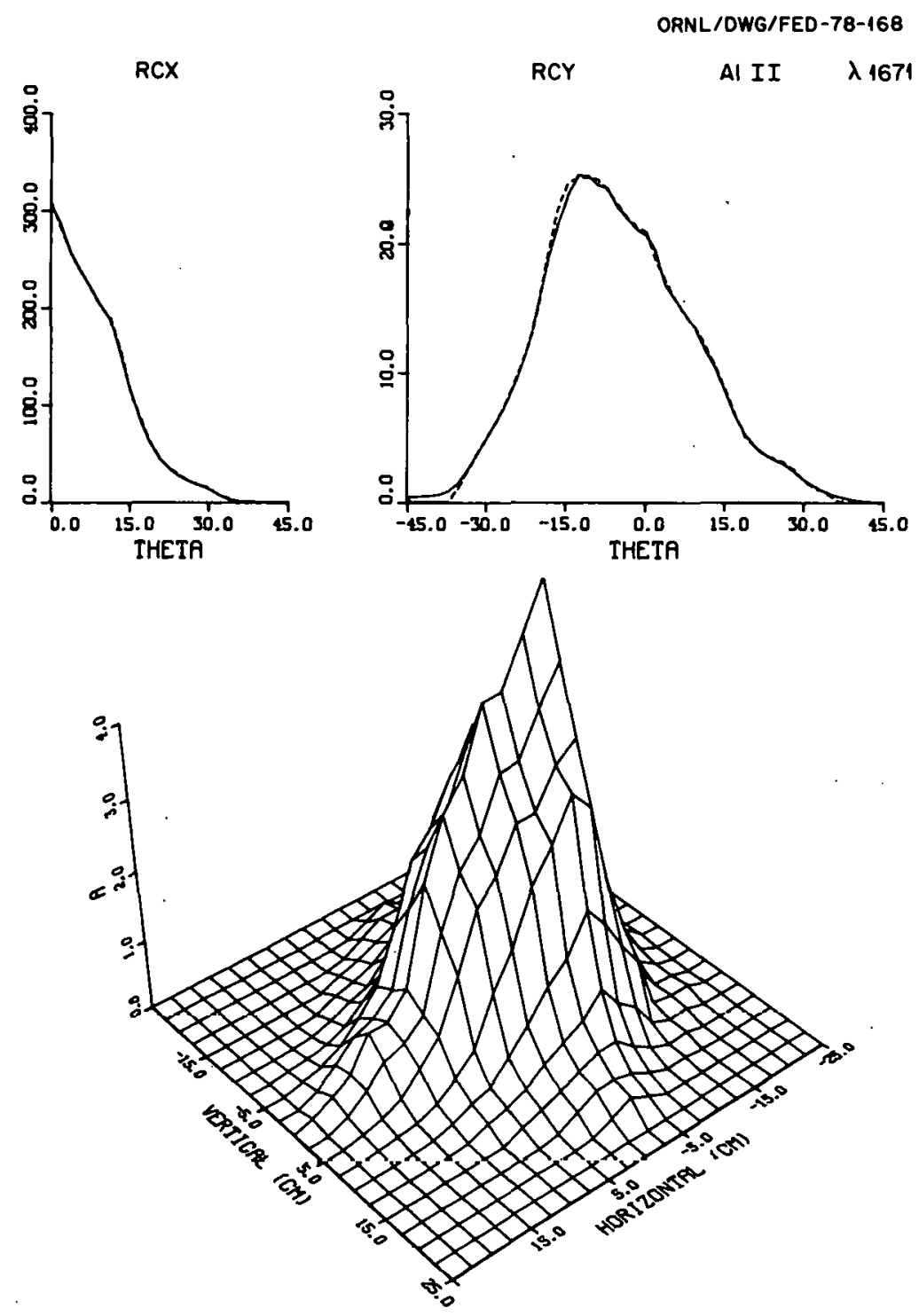

Fig. B.23. Light intensities from A1 II at $\lambda=1671 \AA$ and reconstructed emission distribution in midplane calculated using iterative algebraic reconstruction technique. 
and, for long confinement time, $n_{0}$ at the plasma center must be minimized. The ratio of $n_{0}$ (center) to $n_{0}$ (surface) was determined by the neutral velocity since the mean free path was of the same order of magnitude as the plasma radius.

By measuring the line shape of emitted $H_{\alpha}$ light at $\lambda=6563 \AA$ with a high resolution Fabry-Perot interferometer, it was possible to determine the mean velocity of excited atoms. The observed profile (Fig. B.24) was made up of several fine structure lines, which yielded an estimated mean energy of $0.5 \mathrm{eV}$ when Doppler spread was applied to each of them and the result compared with the observation. This energy was considerably lower than if one assumed that the neutral particles entered the vacuum volume initially as molecules which were dissociated in the surface plasma, producing a flux of hydrogen atoms with a mean energy of $\approx 4-9 \mathrm{eV}$.

The relatively low atom energy observed could be understood by considering the fact that these atoms recirculated between the plasma and the wall many times before being pumped by the diffusion pumps. As a consequence, the measurement implied that atoms were not recombined into molecules in the time they spent on the plasma wall. The details of the chemistry of the hydrogen and the relatively pure surface aluminum metal are not well known, but the low recombination rate implied that atoms were relatively immobile in the metal lattice. This conclusion was reinforced by the large amounts of hydrogen which eventually diffused out of the walls after the experiment was terminated, the amount continuing for a period as long as several hours if the experiment was operated for a long time.

The low atom velocity resulted in a nonuniform atomic density profile in EBT. Without detailed information on the electron density profile, however, the reconstructed light emission profile [at Lyman- $\alpha$ wavelengths, for example (see Fig. B.25)] could not be simply correlated with the detailed spatial distribution of hydrogen. It was possible to estimate $n_{0}(r=0)$, the hydrogen density at the center of the plasma, however, using the absolute calibration of the mirrors and spectrometer carried out at Johns Hopkins University and the peak electron density measured in the toroidal plasma $\left(n_{e}=2 \times\right.$ $10^{12} \mathrm{~cm}^{-3}$ ). It is also important to note that the mirror reflectivities (the mirrors are MgF coated for high reflectivity in the uv) were found to change over a short period of time when exposed to the plasma. To alleviate this problem, the mirrors were rotated so they did not "see" the plasma unless they were being used to take data. In spite of this precaution, when the mirror reflectivities were recalibrated after the data run, they were found to have decreased by a factor of approximately five. During the last few runs at which data were taken, under similar plasma conditions, the count rates were reproducible, and for this reason the last calibration was used in establishing absolute numbers. The value obtained from scans in the stable T-mode was $n_{0}(r=0) \cong 1 \times 10^{10} \mathrm{~cm}^{-3}$, which is at least a tactor of two higher than would be expected trom power balance estimates (see Sect. B.b). Ihe iterative reconstruction technique used in the analysis could overestimate the emission in the center, but it would be surprising if the amount were greater than a factor of two. More work is needed to resolve this apparent difference.

From the observed light distribution, assuming the electron density in the surface plasma region is approximately $3 \times 10^{11} \mathrm{~cm}^{-3}$, the neutral density in this region was estimated as $n_{0} \sim 5 \times 10^{10} \mathrm{~cm}^{-3}$, aga in a factor of two or more higher than anticipated.

\section{B.4.7 TOROIDAL CURRENTS AND FIELD ERROR EFFECTS ON EBT}

Magnetic perturbations in closed field confinement systems can cause field lines to spiral out of the confinement regions and lead to enhanced plasma losses, instabilities, and anomalous convection. ${ }^{6}$ Even though the inherent field errors in EBT were relatively small, their effects on the plasma behavior could have been deleterious because of the long lifetime of the ring-ștabilized plasma and the absence of toroidal currents. 


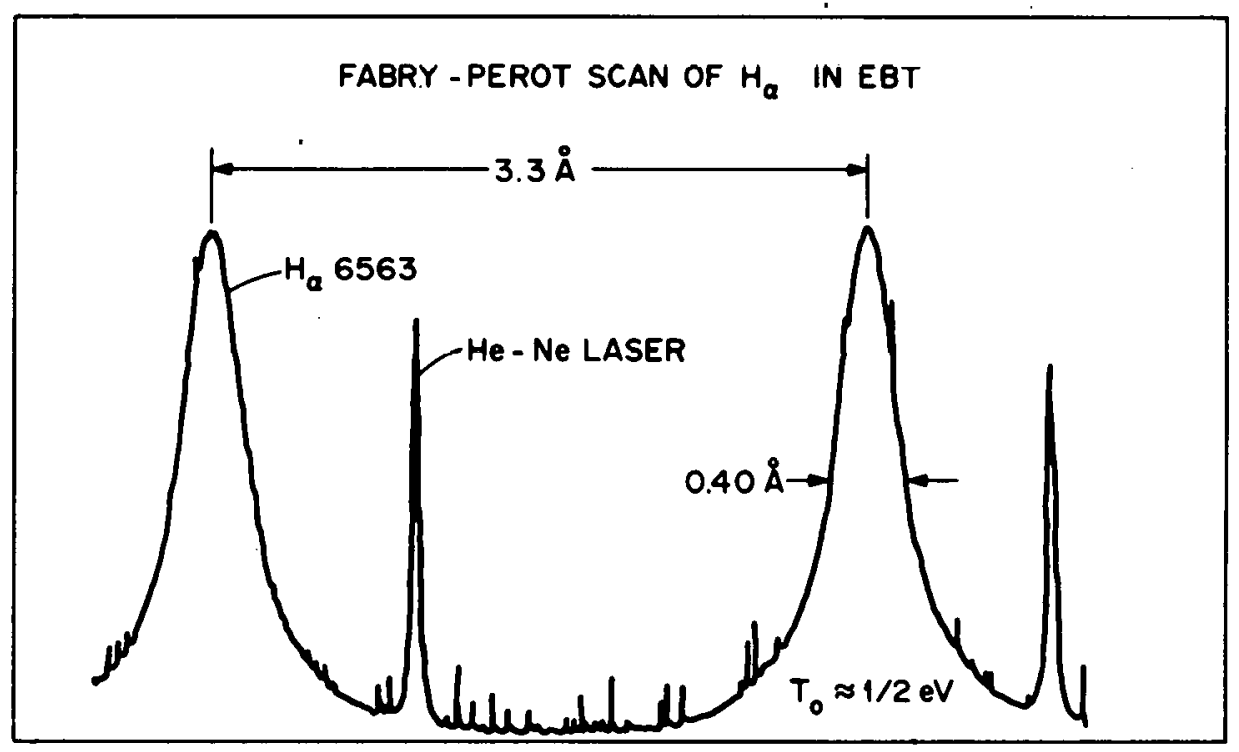

Fig. B.24. Wavelength scan of $H_{\alpha}$ light using Fabry-Perot interferometer. For resolution comparison, the light from a HeNe laser is also shown. 

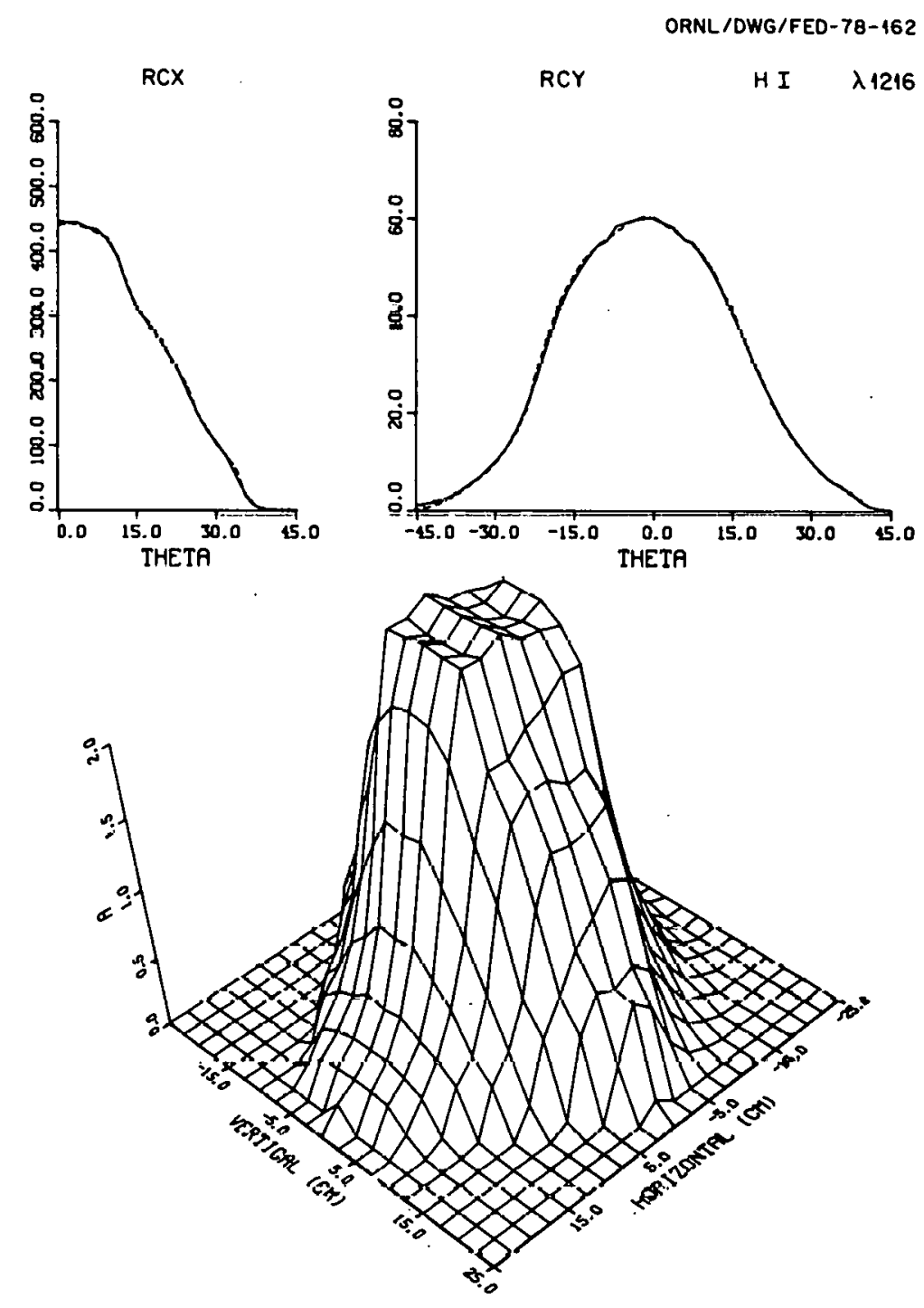

Fig. B.25. Light emission profiles from hydrogen atoms (H 1) at $\lambda=1216 \mathrm{~A}$ (see lig. 13.13 for definitions). 
The vacuum field error was measured in EBT using an electron beam probing technique. The estimated total field error was

$\frac{\Delta B}{B} \cong \frac{\Delta r}{2 \pi R} \leqslant 1.5 \times 10^{-4}$

where $\Delta r$ is the displacement of field lines about the "unperturbed" positions and $R$ is the major radius; measurements were taken for $B \cong 0.2 \mathrm{~T}$. The measured total field error included contributions from the earth's magnetic field $(\approx 20 \%$ in $\Delta B)$, local perturbations, and electron drifts. The field errors resulting from imperfections in coil winding, bus bars, ferromagnetic materials, etc., were found to be quite small (by orders of magnitude) compared with those in many other closed field confinement devices because of the simple coil geometry and careful design and construction. In order to minimize the $n=0, m=1$ component error field, two pairs of current loops were wound around the torus as schematically shown in Fig. B.26. The loops were about $50 \mathrm{~cm}$ from and parallel to the minor axis; each loop consisted of a 20-turn coil capable of carrying a current up to $30 \mathrm{~A}$. The transverse magnetic field generated by these loops could be used to cancel the average error field or to increase the field error, thus permitting studies of plasma stability and confinement with the error field as a parameter.

The plasma was found to exhibit a small net toroidal current ( $2100 \mathrm{~A}$ ) related to the field error effect. This toroidal current was experimentally monitored by two toroidal coils surrounding the torus (see Fig. B.26); the resulting measurements are shown in Fig. B.27. The effect of magnetic field error cancellation is evident in this figure from the large reduction of toroidal current. The residual global error was taken to be zero at the minimum toroidal current; thus, the original error field $\Delta B_{0}$ could be estimated from the current required for null toroidal current,

$\frac{\Delta B_{0}}{B} \cong-\Delta B_{1} \cong \frac{I}{2 \pi r B} \cong 3 \times 10^{-4}$

which is close to the integral vacuum field error measured by the electron beam probe.

Assuming that the error field produces an additional radial component to the otherwise axisymmetric bumpy magnetic field, a toroidal current in the plasma will be driven by the component of radial electric field lying along the magnetic flux lines,

$j_{\|}=\frac{E_{\|}}{n_{\|}}=\frac{E_{r}}{n_{\|}} \frac{\Delta B}{B}$,

where $n_{\|}$is the resistivity $n_{\|}=\sqrt{m} e^{/ 2 \pi}\left(Z e^{2} / 32 \varepsilon_{0}\right)\left[\ell n \Lambda /\left(k T_{e}\right)^{3 / 2}\right]$. Integrating $j_{\|}$across the plasma cross section yields the toroidal current,

$$
\begin{aligned}
I & =\int j_{\|} d \theta \\
& =\int_{0}^{a} r d r \int_{-\pi / 2}^{\pi / 2} d \theta \frac{E_{\perp}(r)}{2 \pi \eta_{\|}(r)}\left(\frac{1}{R_{0}+r \cos \theta}-\frac{I}{R_{0}-r \cos \theta}\right) \Delta r \cos \left(\theta-\theta_{0}\right) \\
& \cong \frac{1}{2}\left(\frac{a}{R}\right)\left(\pi a^{2}\right)\left\langle\frac{E_{\perp}}{\eta_{\|}}\right\rangle \frac{\Delta B}{B},
\end{aligned}
$$

where the directional cosine of the field error, $\cos \theta_{0}$, and the sign of current density, $j_{\|}=j_{\|}(r, \theta)$, have heen taken into account explicitly. Given a typical set of EBT plasma parameters $\left[T_{c} \cong 200 \mathrm{eV}\right.$, $E_{\perp}(r) \approx k T e^{/ e a}$, and $\left.\Delta B / B \cong 1 \times 10^{-4}\right]$. Eq. (8) gives a total toroidal current of $\approx 150 \mathrm{~A}$, which is in 
10.6 GHZ (LORH) MICROWAVE FEED (1)

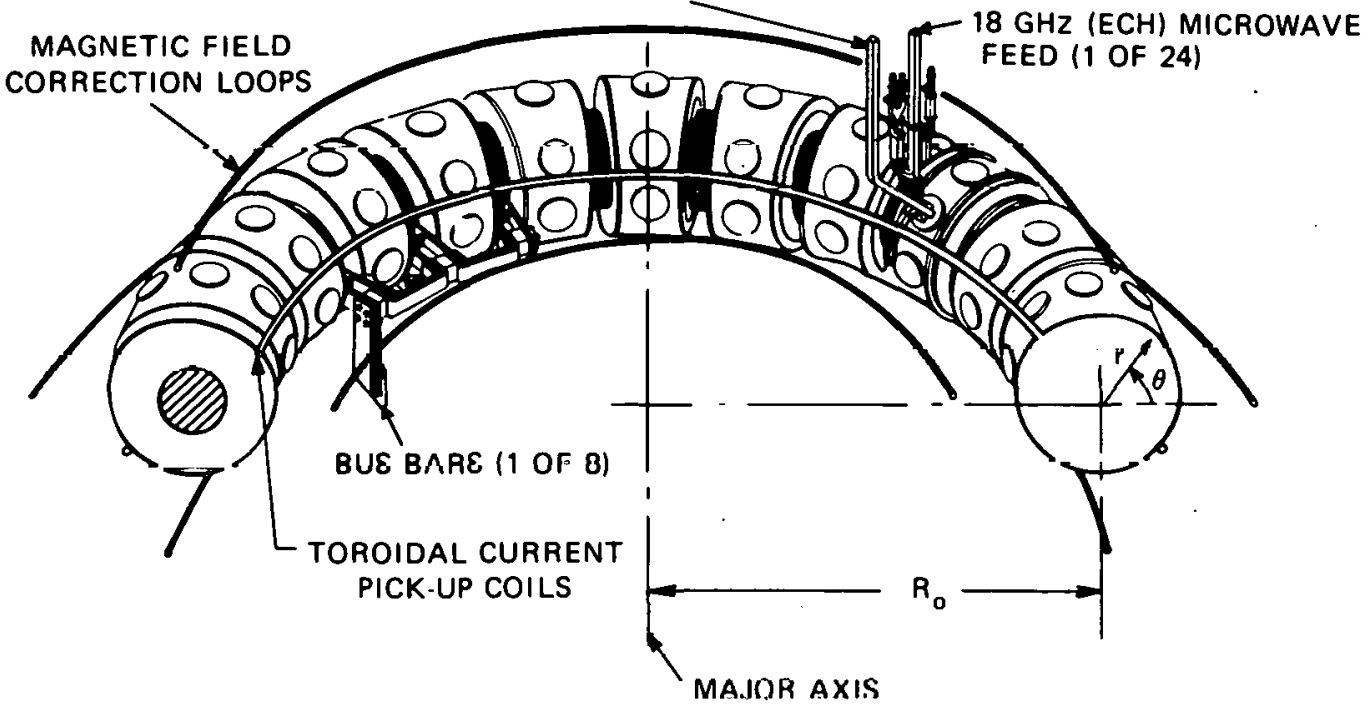

Fig. B.26. Schematic section of EBT-I showing locations of quadripolar correction coils and toroidal current pickup loop.

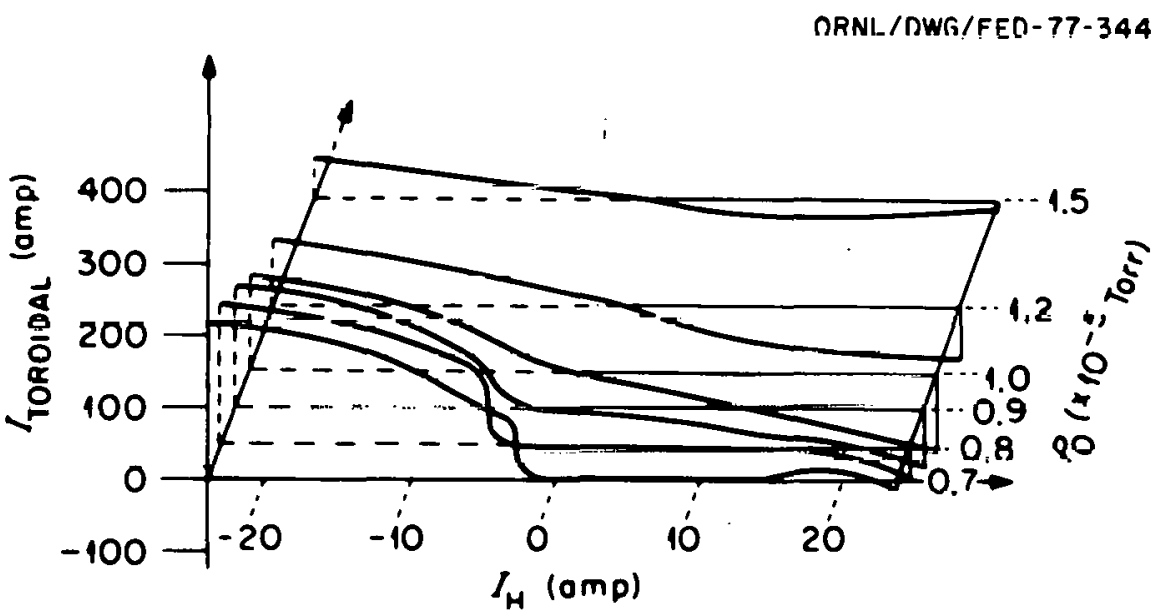

Fig. B.27. Turotdal current measured with pickup loop as a tunction of horizontal error field current for plasmas produced at various pressures. 
good qualitative agreement with measurements. Thus, a strong relationship between field error cancellations and toroidal current magnitude could be inferred.

A more detailed comparison of the data in Fig. B.27 with results of this model for the source of the observed toroidal current showed additional elements of agreements. At high pressures the observed current varied linearly with error field as predicted [Eq. (8)]. These conditioris constituted $C$-mode states with highly collisional plasmas where the model should be quite applicable. At lower pressures ( $T$-mode), the plasma became less collisional, and the existence of annuli stabilized the toroidally confined component. The toroidal current was found to be significantly reduced over a significant range of variation of $\mathrm{I}_{\mathrm{H}}$.

A tentative explanation for the observed low current has been suggested. Given that the toroidal plasma is less collisional in the T-mode, most of the particles would be circulating on closed drift surfaces when field errors are small. Thus, very few charges would leave the toroidal column along the magnetic lines of force and Eq. (7) would not be applicable. The plasma would carry very little current until the field error becomes large enough so that an appreciable fraction of the drift surfaces intersect the wall. A simple calculation shows that this transition can occur within the range of $f i e l d$ errors found experimentally; $\delta B / B \sim 0.6-1 \times 10^{-3}$.

The effects of magnetic field errors on plasma stability and confinement in EBT are demonstrated in Fig. B.28 for plasma in the stable T-mode. The net toroidal current, the averaged plasma density, the averaged density fluctuation amplitude, and the plasma potential measured at the center and near the annulus are plotted together with the horizontal field error as functions of the current $I_{H}$ applied to the field correction coil. The correlation of the abrupt change in the plasma observations at a critical error field (in this case $I_{H} \sim-8 A$ ) is particularly striking. Within the range of error fields where the toroidal current was small $\left(-10 \mathrm{~A}<\mathrm{I}_{H}<+15 \mathrm{~A}\right)$, the T-mode properties agreed with those described in earlier sections (negative central potential, low plasma fluctuations, increase of $\approx 30 \%$ in ne compared to that with large error fields present).

It is possible to evaluate the influence of error fields on neoclassical diffusion for sufficiently small values of error fields. If the radial drift due to the error field is added to the radial velocity component in the usual drift kinetic equation, the result is

$v_{r}=v_{0} \sin \theta+v_{\|} \frac{\Delta B_{r}}{B_{r}}$

where $v_{0}$ is the vertical drift due to toroidal field curvature. Fourier analyzing and linearizing the drift kinetic equation lead to an expression for the electron distribution function, $f_{m n}$, which may be used to calculate the radial flux, $\Gamma_{r}$,

$\Gamma_{r}=\frac{d \theta d \phi}{(2 \pi)^{2}} f_{1} v_{r}$

The new diffusion coeffictẹnt, $D^{\star}$, can be evaluated using

$\Gamma_{r}=D^{*} \nabla_{r} n+{ }_{r_{r}}^{*} \mathrm{nE}_{r}$,

with $\mu^{*}=q D^{\star} / k T_{e}$. One obtains for $D^{\star}$

$D^{\star} \cong \frac{v_{0}^{2}}{2} \frac{v}{v^{2}+\left(v_{D} / r\right)^{2}}+\frac{\left\langle v^{2}\right\rangle}{3} \sum_{m, n}\left(\frac{\Delta B}{B, n}\right)^{2} \frac{v}{v^{2}+m\left(v_{D} / r\right)^{2}+\left[n\left(v_{\|} / R\right)\right]^{2}}$. 


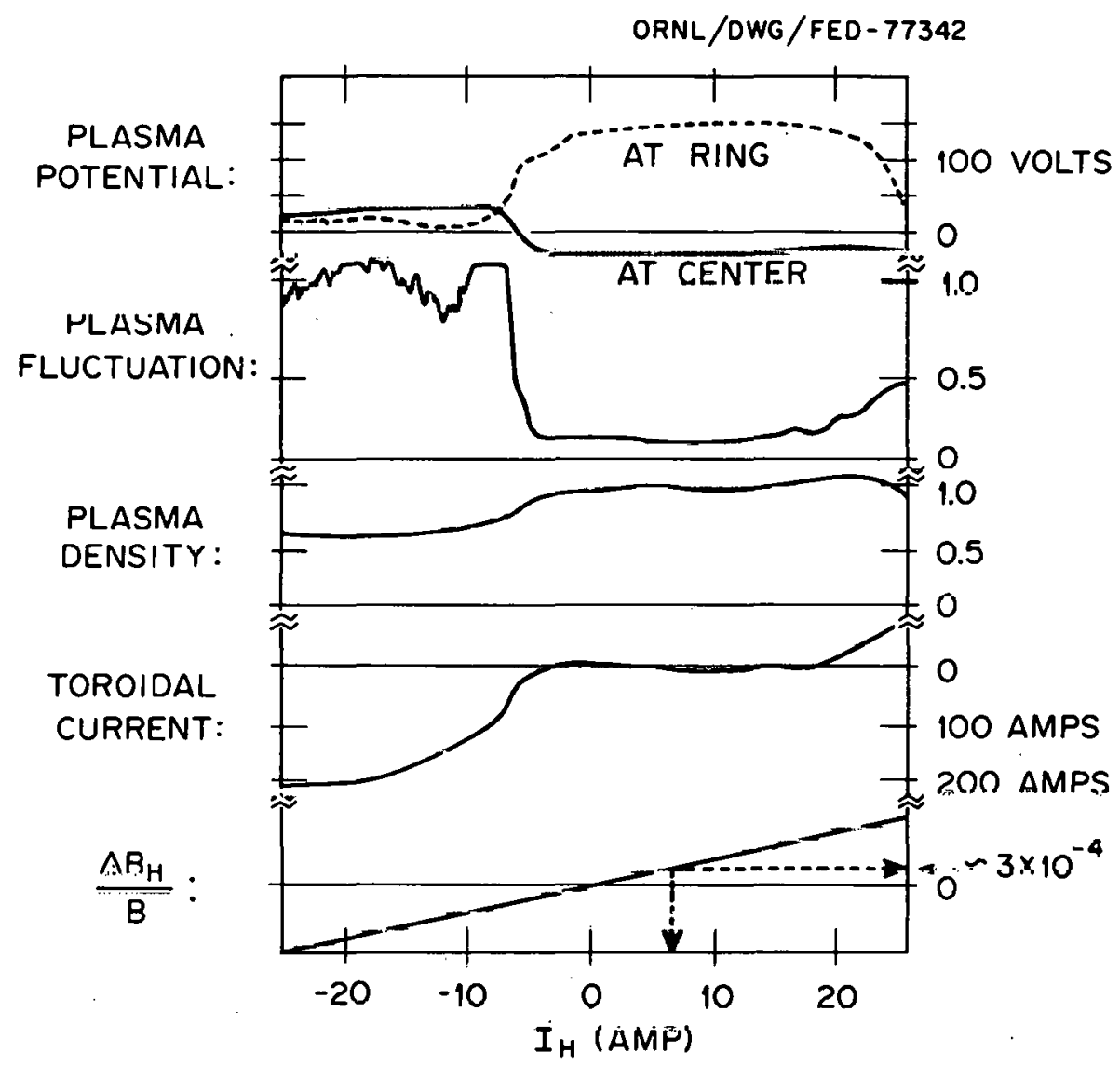

Fig. B.20. Correlations of several parameters measured simultaneuusiy as the horizontal field error scans in the range of $\pm 1-2 \times 10^{-3}$. The probable system global error is estimated to be $3 \times 10^{-4}$, as indicated by the arrowliead. 
The first term in Eq. (12) is the usual neoclassical diffusion ${ }^{7}$ rate due to toroidicity (asymmetry), while the second term is the result of the radial magnetic perturbation. 8 From Eq. (12), for $n \neq 0$ the magnetic field error effect on diffusion is negligible, and for the $m=1, n=0$ component the error field effect could be comparable to toroidicity only when

$\frac{\Delta B}{B} \geq\left(\frac{\Delta B}{B}\right)_{C r} \cong \sqrt{3 i 2} \frac{\rho}{R} \sim 10^{-4}-10^{-3}$

for EBT-I plasmas. Field errors, therefore, simply modify the usual neoclassical diffusion rate by the factor

$D^{*}=D_{\text {neoclassical }}\left[1+u\left(\frac{R}{\rho} \frac{\Delta B}{B}\right)^{2}\right]$.

The value $\Delta B_{r} / B$ for which the diffusion coefficient is changed only negligibly by the error fields is inside the range of values of $\Delta B / B$ for which the toroidal currents were observed to be small - $i . e$., electron transport was most likely unaffected in this range of $I_{H}$. Consistent with this fact was the relatively small variation of plasma properties between $-10 \mathrm{~A}$ and $+15 \mathrm{~A}$.

Outside this range, the abrupt change in parameters indicated that a new mechanism was taking place. The high fluctuation level was probably a clue to the effect - perhaps toroidal current induced instabilities. These regimes have not been thoroughly explored, nor has the possibility of spatially localized toroidal currents, but future plans include these studies.

\section{REFERENCES}

1. R. A. Dand1, H. 0. Eason, A. C. England, G. E. Guest, C. L. Hedrick, and J. C. Sprott, The ELMO Bumpy Torus Experiment, ORNL/TM-3694, Oak Ridge, Tennessee (November 1971).

2. EBT Experimental Group, Summary of EBT-I Experimental Results, ORNL/TM-6457, 0ak Ridge, Tennessee (to be published).

3. M. Bacal, A. Truc, H. J. Doucet, H. Lamain, and M. Chrétien, Nucl. Instrum. Methods 114, 407-409 (1974); M. Bacal and W. Reichelt, Rev. Sci. Instrum. $45,769-772$ (1974).

4. F. C. Jobes and R. L. Hickok, Nucl. Fusion 10, 195 (1970); P. L. Colestock, K. A. Connor, R. L. Hickok, and R. A. Dandl, Phys. Rev. Lett. $\underline{40}, 1717$ (1978).

5. An early review of various algebraic reconstruction techniques as applied to medical tomography is presented in ItEt Irans. Nucl. Sci. NS-2I $(19 / 4)$.

6. T. Jernigan, J. Rudmin, and D. M. Meade, Phys. Rev. Lett. 26, 1298 (1971); M. Okabayashi and

R. Freeman, Investigation of Magnetic Field Errors in the Levitated Spherator, MATT-870, Princeton, New Jersey (November 1977).

7. L. M. Kovrizhnykh, Zh. Eksp. Teor. Phys. [Sov. Phys.-JETP] 29, 475 (1969).

8. D. M. Meade and R. J. Fonck, Phys. Fluids 16, 1654 (1973). 
THIS PAGE

\section{WAS INTENTIONALLY \\ LEFT BLANK}


APPENDIX C

EBT-II MAGNETICS DESIGN 
THIS PAGE

\section{WAS INTENTIONALLY LEFT BLANK}


The selection of the EBT-II major radius, number of sectors, mirror ratio, and magnet design is based on maximizing vacuum field particle confinement within the constraints of microwave power, superconducting magnet technology, and machine cost. Although finite beta and ambipolar electric fields improve the confinement of all particles except the very high energy passing particles, it is not possible to include these effects at this design level. Hence, the confinement properties of the vacuum magnetic field are used as the basis for choosing one design over another.

The toroidal curvature of the magnetic field in EBT devices results in an inward shift of particle drift orbits from the minor axis toward the major axis of the torus. The shift is largest for the transitional and passing particles, i.e., those having a large component of velocity parallel to the magnetic field. This conceptual design study incorporates a method of improving particle confinement in EBT-II through the addition of low current supplementary toroidal field coils called aspect ratio enhancement (ARE) coils. By varying the current in the ARE coils, the plasma geometry and effective aspect ratio can be varied, with the effect of either spoiling or enhancing plasma confinement.

Enhanced particle confinement and the increased flexibility for plasma experiments afforded by ARE coils are discussed in some detail.

In these magnetic field design studies, the plasma volume was taken to be approximately $1 \mathrm{~m}^{3}$, and single particle confinement was optimized via particle drift orbit studies which included the perturbations caused by practical design considerations. Particle drift orbits were calculated for a 36-sector device and compared with those for a 48-sector device. In both cases, the mirror ratio, $M$, was $\approx 2$. The results for 36 sectors are presented in Fig. C.1, where the area of the last closed drift orbit that does not intercept the limiter (mirror coil case) is plotted as a function of the cosine of the pitch angle. The solid and dashed curves correspond to plasma volumes of approximately $2.3 \mathrm{~m}^{3}$ and $1.25 \mathrm{~m}^{3}$, respectively. Note that no passing particles are confined in the smaller 36-sector device. The results for 48 sectors (without aspect ratio enhancement), shown as the solid curve in Fig. C.2, correspond to a plasma volume of about $1 \mathrm{~m}^{3}$ and demonstrate that good passing particle confinement can be obtained with 48 sectors and one half the microwave power required for a 36-sector device having similar confinement properties.

Since devices heated with electron cyclotron heating $(E C H)$ require $\omega_{c e}>\omega_{\text {pe }}$, a resonant magnetic field of $4.3 \mathrm{~T}$ (corresponding to a cutoff plasma density of $1.5 \times 10^{14} \mathrm{~cm}^{-3}$ ) was chosen along with the corresponding microwave heating source frequency of $120 \mathrm{GHz}$. The bulk heating power is most effective when it resonates around the geometric mean mod-B surface, i.e., $B_{r}=B_{0} \sqrt{M}$, where $M$ is the mirror ratio and $B_{O}$ is the field on axis in the midplane. If a mirror ratio of $2: 1$ is chosen, then the corresponding minimum and maximum fields on axis are $3.0 \mathrm{~T}$ and $6.0 \mathrm{~T}$, well within the range of conventional niobiumtitanium (NBTi) superconducting magnet technology. At this field level a significant scaling test is possible; reducing the field does not lead to enough reduction in cost to justify limiting the range of possible scaling tests.

Since enhanced particle confinement is obtained only when the ARE coils are operated in the reverse current mode (i.e., the magnetic field generated by the ARE coils subtracts from the mirror coil field), a strong mirror coil field is highly desirable. Several alternate magnetics designs with mirror ratios larger than 2:1 were examined. Although the vacuum field particle confinement was slightly better than for $M \approx 2$, it was not possible, within the constraints on current density and maximum field at the superconducting coil windings, to obtain the high midplane field $(3.0 \mathrm{~T})$ that is desirable for EBT-II. It should be noted in this regard that one of the effects of ARE coils is an increase in the mirror ratio with attendant improvements in particle confinement that are distinctly superior to those obtained by simply increasing the mirror ratto of the device without adding ARE coils.

Fairly conservative design constraints for the superconducting mirror coils are assumed. The two most important of these for optimization of particle confinement are the current density $\left(\leq 10,000 \mathrm{~A} / \mathrm{cm}^{2}\right)$ 


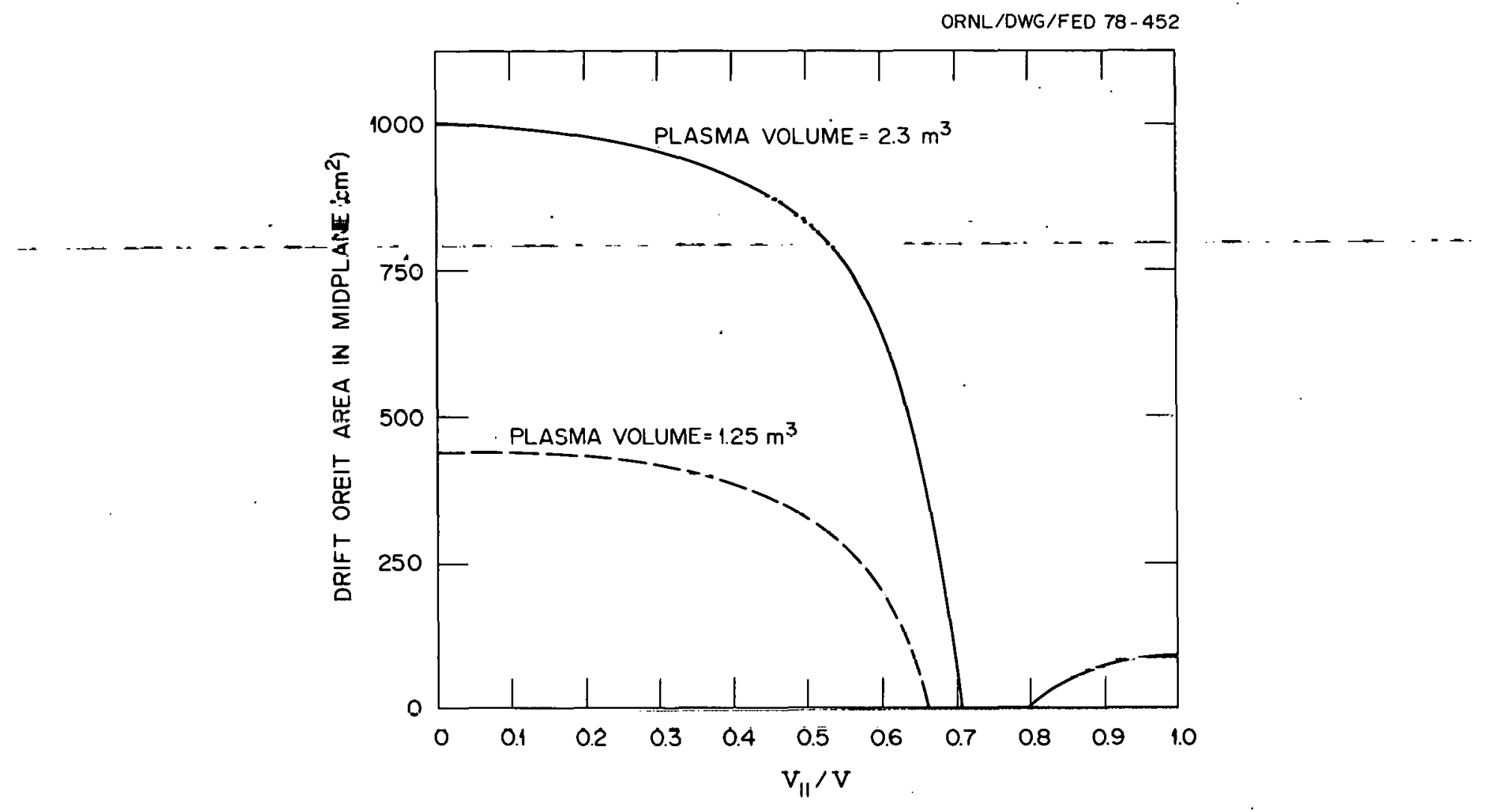

Fig. C.1. Area of last closed drift orbit that does not intercept the limiter (mirror coil case) plotted as a function of the cosine of the pitch angle for a 36-sector device. The solid and dashed curves correspond to plasma volumes of $2.3 \mathrm{~m}^{3}$ and $1.25 \mathrm{~m}^{3}$, respectively. The mirror ratio is approxillately 2 . 


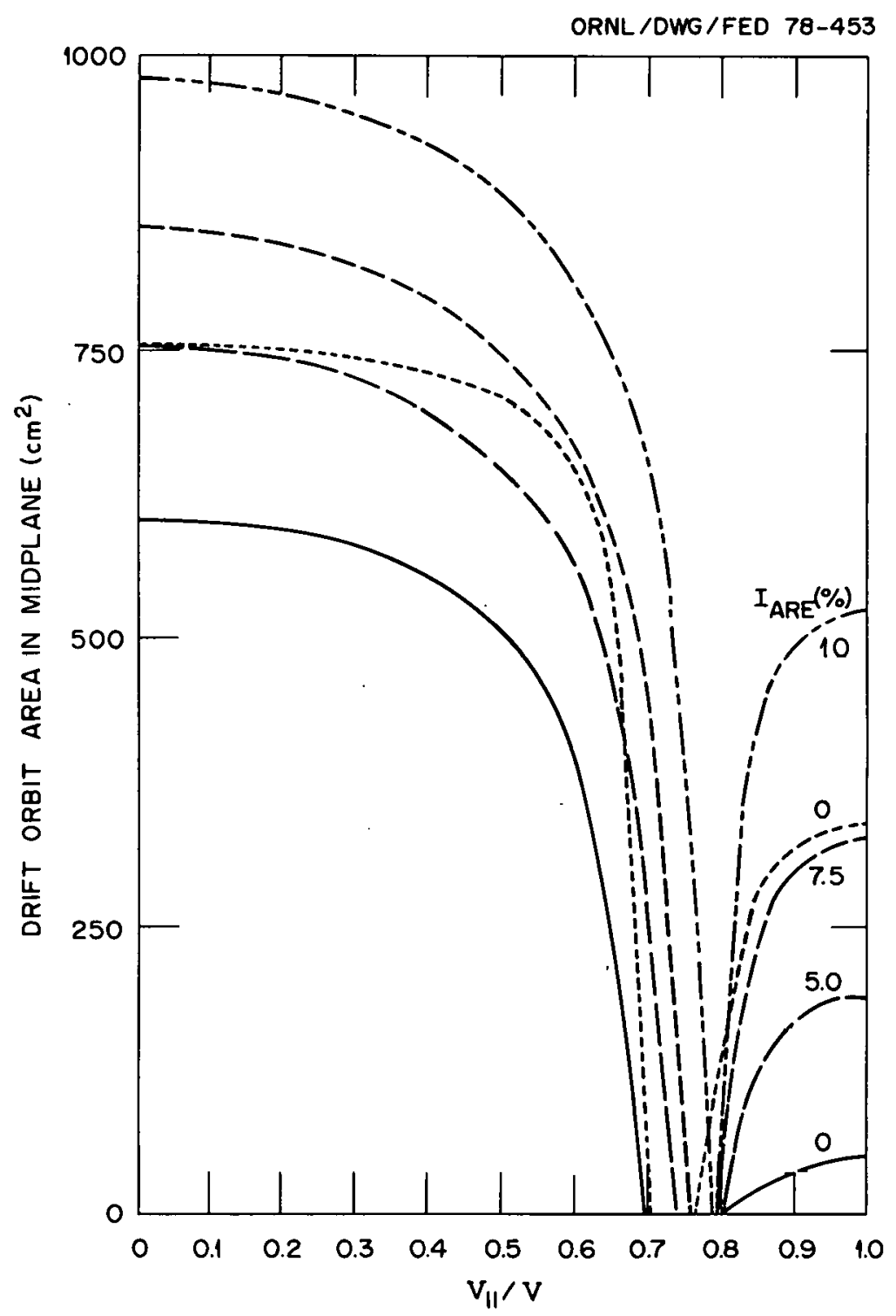

Fig. C.2. Area of last closed drift orbit that does not intercept the limiter (mirror coil case) plotted as a function of the cosine of the pitch anglc for a 48-scctor devicc with several values of $I_{A R E}$ (solid and long dashed curves). The plasma volume and mirron rations are approximately $1 \mathrm{~m}^{3}$ and 2 . respectively. Results for a 96-sector device with major radius of $10.4 \mathrm{~m}$ and no ARE coils are also plotted as the small dashed curve. 
and the maximum field at the coil windings $(\leq 8.0 \mathrm{~T})$. The inside radius of the coil was chosen to yield the maximum "clear bore" for collisionless toroidal particle circulation. The maximum field at the coil windings increases as the ratio of the radial build of the coil to its length increases. Since the ARE coils must fit between the mirror coil cans on the inside of the torus (see Fig. C.3), it was desirable to make this ratio as large as possible, subject to the constraint (noted above) on the maximum field at the coil windings and consistent with $M \approx 2$.

The desired particle confinement properties, magnetic field strength, and plasma volume dictate roughly the minimum required major radius for the torus. Further adjustment was made to permit installation of planar ARE coils in the positions shown in Fig. C.3. This increase in the major radius $R_{T}$ from the minimum required value of $\approx 4.5 \mathrm{~m}$ to $5.2 \mathrm{~m}$ also results in a decrease in required mirror coil current density. With the larger major radius, the coil can have a larger cross-sectional area and much better operational reliability for a given field strength and geometry.

In this design study the ARE coils are, for simplicity. taken to be normal conducting, water cooled copper coils. Work is now in progress to ascertain the feasibility, advisability, and relative cost of making the ARE coils superconducting. Although superconducting ARE coils would require $x-$ ray shielding, the cross-sectional area of the conductor would be smaller than that of the normal coils. Hence, the conductor positions would probably be roughly the same as those of the normal conducting ARE coils shown in Fig. C.3, and the drift orbit confinement results discussed below would change very little.

The effect of the ARE coils on the field geometry is illustrated in Fig. C.4, where mod-B contours and field lines in the equatorial plane of an EBT-II sector are plotted for ARE coil currents, I ARE (current in ARE coils/current in mirror coils), of 0 and $7.5 \%$. Note that the ARE coils very nearly symmetrize the mod-B contours about the minor axis in the midplane. This symmetrization permits an increase of $21 \%$ in the radius of the hot electron rings and a corresponding increase of almost $50 \%$ in the volume of mirror-confined plasma. Another important effect of ARE coils is to lengthen the magnetic field lines on the inside of the torus relative to those on the outside, resulting in significantly enhanced confinement of the transitional and passing particles (see Figs. C.2, C.5, and C.6). Note, in addition, that the constriction of the passing particle bore caused by inward protrusion of the mirror coil $x$-ray shield is much less severe with the increased field line curvature obtained with ARE coils.

The increase in mirror ratio discussed above is also apparent in Fig. C.4. The case with $I_{A R E}=0$ corresponds to $M=1.95$ and the case with $I_{A R E}=7.5 \%$ to $M=2.25$. A current density of $6650 \mathrm{~A} / \mathrm{cm}^{2}$ in the mirror coils yields a magnetic field strength on axis in the midplane (coil plane) of $3.0 \mathrm{~T}$ (5.85 T) with $I_{\text {ARE }}=0$. With $I_{\text {ARE }}=7.5 \%$ the midplane (coil plane) field drops to $2.5 \mathrm{~T}(5.62 \mathrm{~T})$.

Detailed effects of ARE coils on particle drift orbits for $v_{\|} / v=0$ (particles trapped near the midplane) and $v_{\|} / v=1.0$ (extreme passing particles) are shown in Figs. C.5 and C,6. The current in the ARE coils is varied from $I_{A R E}=0$ (mirror coil field only) to $I_{A R E}=10 \%$. As the current is increased, a dramatic shift in the center of the drift orbits back toward the mirror axis of the torus is observed, as is the increased plasma radius permitted by the ARE coils.

The area of the last closed drift orbit that does not intercept the limiter is plotted for all values of $v_{\| l} / v$ in Fig. C.2. The drift orbit areas for a 96 -sector torus with a major radius of $10.4 \mathrm{~m}$ are given for comparison. The three principal effects of ARE coils on particle confinement are illustrated in this graph: (1) centering of the trapped particle drift orbits about the mirror axis and the increase in permitted plasma radius, resulting in an increase of up to $65 \%$ in the volume of mirrorconfined plasma; (2) a dramatic decrease in the width of the "loss cone" in velocity space (in fact, a much larger decrease than could be obtained with an increase by a factor of two to three in the mechanical aspect ratio, i.e., number of sectors and major radius); and (3) up to a factor of ten increase in 


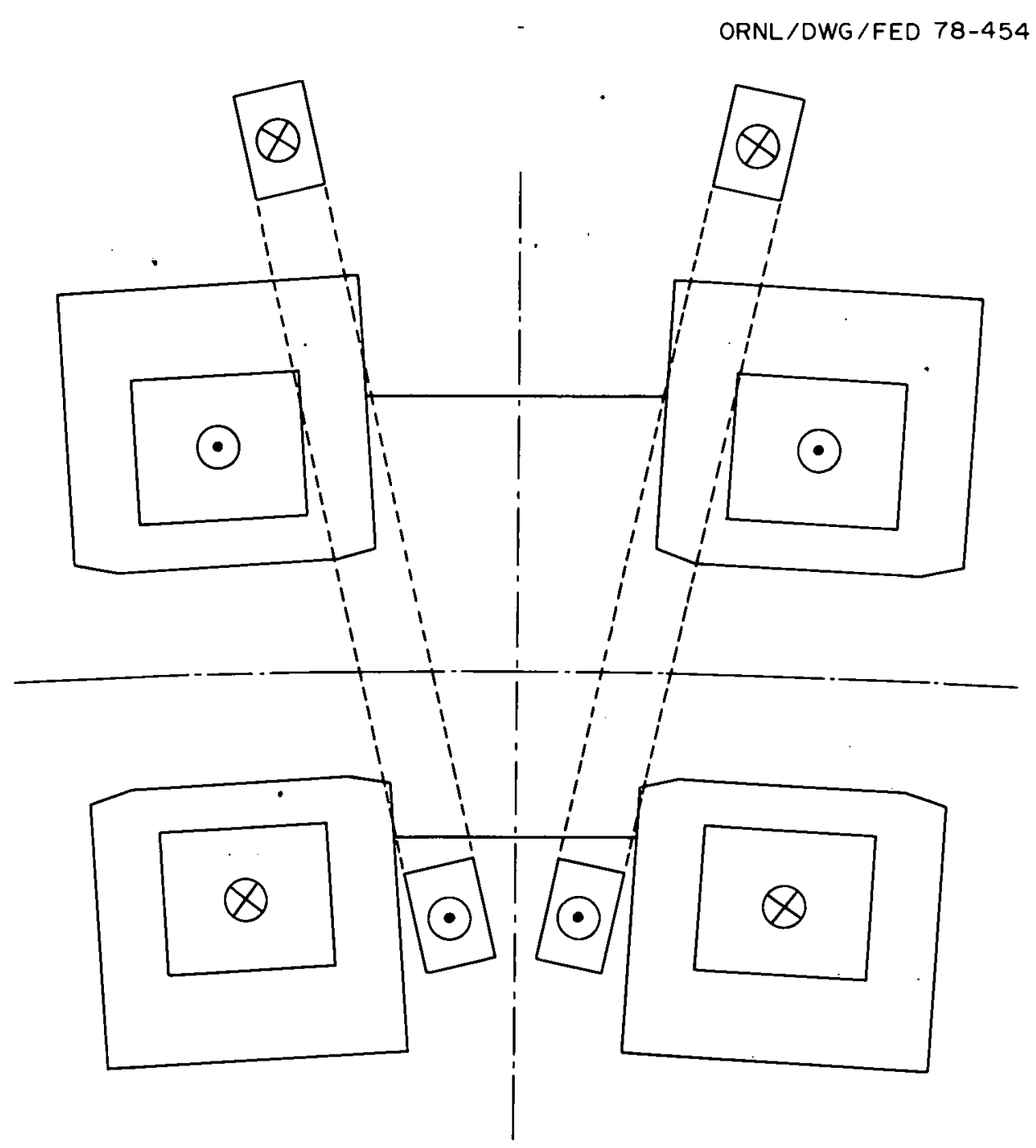

Fig. C.3. Sector of EBT-II in the equatorial plane displaying position of the superconducting mirror. coils and ARE coils. Dots and crosses indicate polarity of currents when in the enhancement mode. 

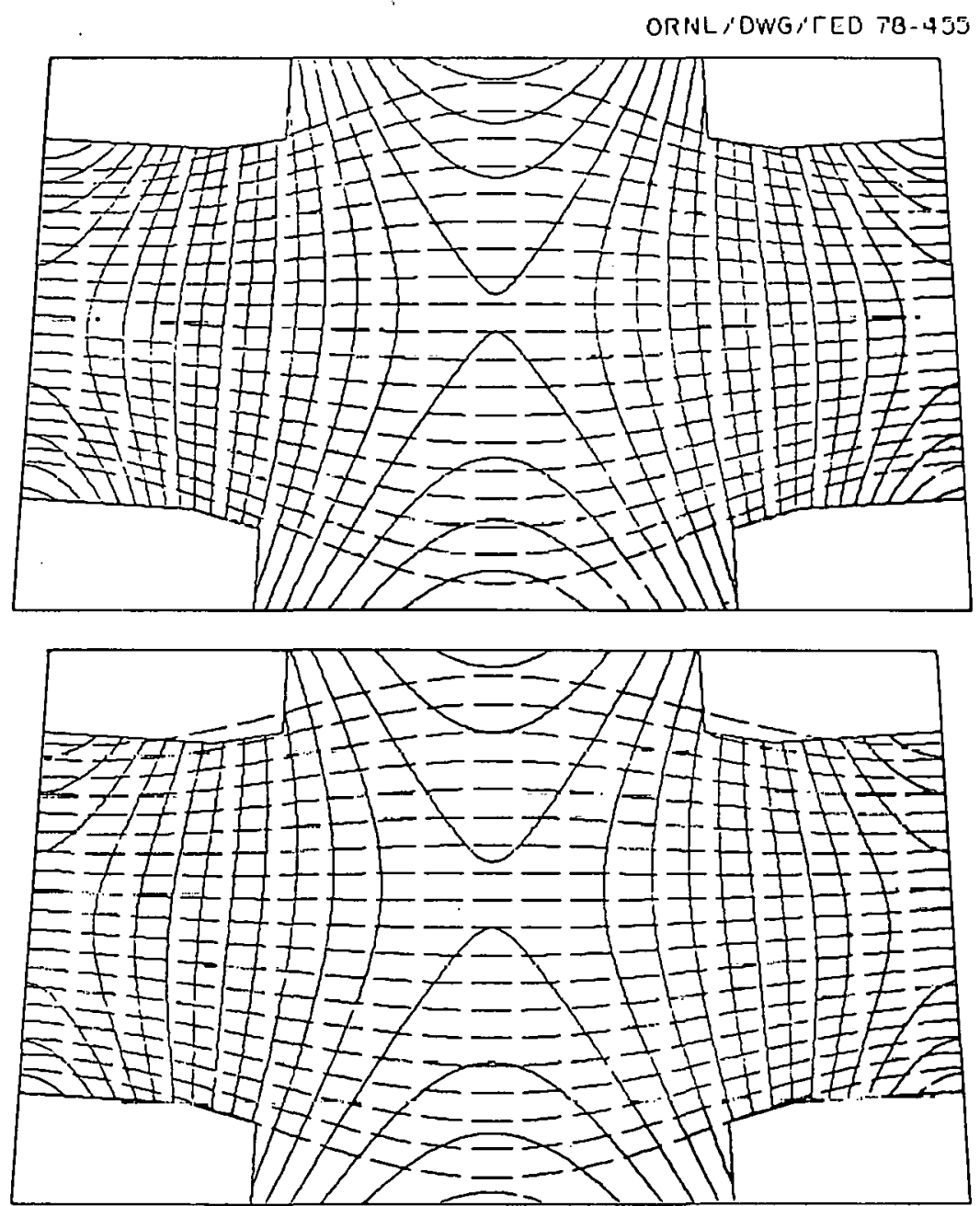

Fig. C.4. Effect of ARE coils on field geometry. Mod-B contouits (dashed 1 ines) dind rield l lnes (sulld lines) in the equacorial plane are plotted for ARE coil currents I ARE of zero (top) and $7.5 \%$
(bottom). 


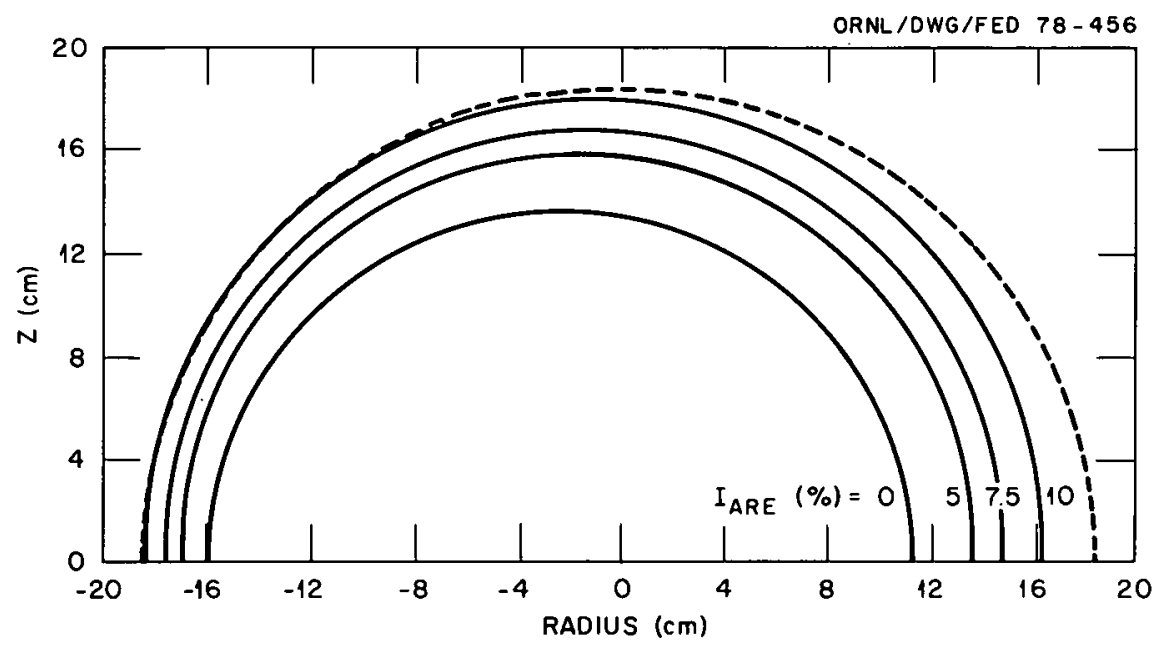

Fig. C.5. Effects of ARE coils on particle drift orbits for $v_{\|} / v=$ 0 (particles trapped near midplane). I $\mathrm{I}_{\text {ARE }}$ varied from 0 to $10 \%$. The dashed lines outl ine the mirror coil case.

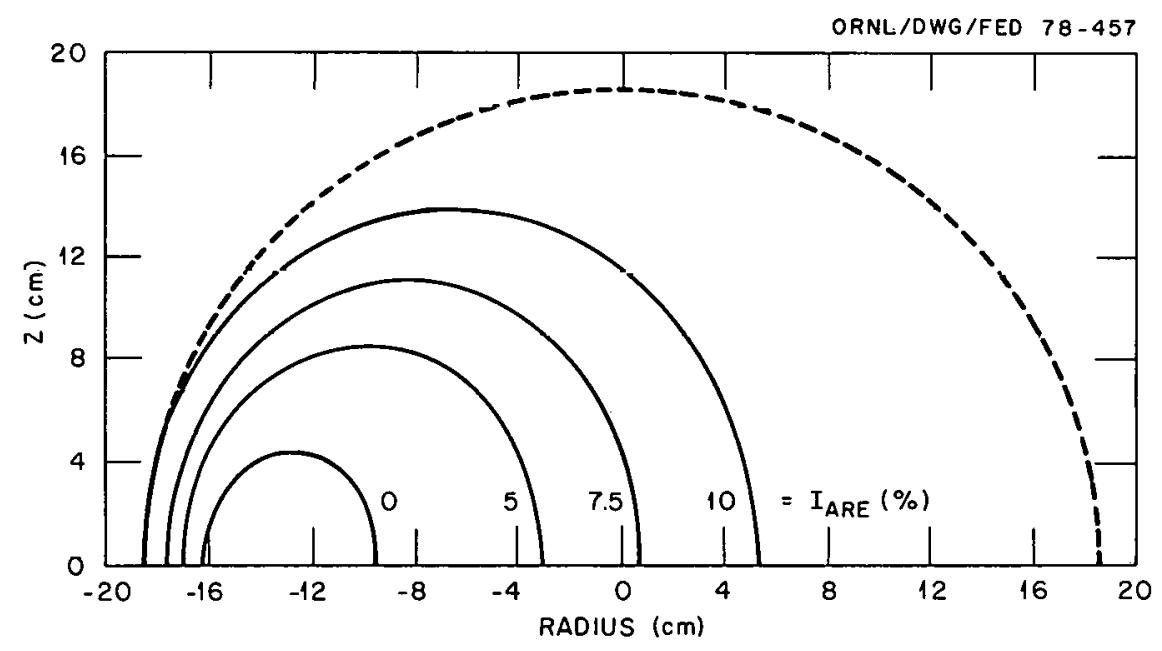

Fig. C.6. Effects of ARE coils on particle drift orbits for $v_{\| l} / v=$ 1:0 (extreme passing particles). I ARE varied from 0 to $10 \%$. The dashed lines outline the mirror coil case. 
the passing particle bore. This increase in the bore available for collisionless toroidal particle circulation is particularly significant, since high energy particles of this type are little influenced by weak electric fields and finite beta.

These curves show that a modest ARE coil current (7.5-10\%) can result in an aspect ratio enhancement factor of two or more. This means that the confinement properties of the device are as good as those of a device having twice the mechanical aspect ratio or volume. 
APPENDTX $\mathrm{N}$

NEUTRAL BEAM HEATING FOR EBT-II 
THIS PAGE

\section{WAS INTENTIONALLY LEFT BLANK}


The addition of neutral beam heating to EBT-II constitutes Phase 2 of the experiment and will not occur until most of the Phase I experiments are complete. Therefore, the design criteria for the neutral beams do not need to be as firm as those for the other systems at this time. Every effort has been made to plan for a neutral beam injection system which will allow achievement of significant plasma ion energy enhancement at a reasonable cost, but the details are left somewhat vague to permit the input of confinement results from Phase I experiments in order to optimize the design parameters of the neutral beam system.

Neutral beam injection perpendicular to the field lines (vertically down as shown in Fig. D.1) is particularly well suited for an EBT device. This geometry provides good mechanical access for the beam lines, causes injected particles to be trapped with large pitch angles ( $i . e ., v_{1} / v \cong 1$ ), and permits relatively low beam energies (i.e., $E_{b} \cong 20 \mathrm{keV}$ ).

The target plasma density obtainable using microwave heating and ARE coils will be high enough to give good trapping efficiency to the injection system, and the confinement time is expected to be sufficiently long to allow significant plasma heating from several hundreds of kilowatts of neutral beam power. In this appendix some aspects of the beam-plasma system are examined to show that a neutral hydrogen beam heating system based on $20-\mathrm{keV}$ injectors at an input power level of 1 MW would be adequate for EBT-II Phase 2 experiments.

\section{1 PLASMA PARAMETERS RELEVANT TO NEUTRAL BEAM HEATING}

Perpendicular injection is the most attractive injection geometry from both physics and engineering viewpoints, since injectors and beam dumps can be located in areas not needed by other equipment. The presence of ARE coils on EBT-II leaves the choice of vertical injection, shown in Fig. D.1, as the most reasonable arrangement for the injection geometry.

With the injection geometry fixed, the density profile along the injection path in the target plasma can be determined from radial transport calculations. 1 In the following discussion, either a constant radial density profile or a parabolic one is assumed. Numerical values stated for density will be those on axis in a cavity midplane.

In order to calculate the stored energy and total number of particles in the plasma, it will be assumed that the effective plasma volume $\left(V_{p}\right)$ is $1 \mathrm{~m}^{3}$, a reasonable value for EBT-II, based on a midplane plasma radius of $15 \mathrm{~cm}$. The following heating power requirements relate only to the toroidal core plasma.

The assumed density and temperature values for the target plasma of EBT-II Phase I are $n \approx 5 \times 10^{13}$ $\mathrm{cm}^{-3}, \mathrm{~T}_{j} \approx 3 \mathrm{keV}$, and $T_{\mathrm{e}} \approx 6 \mathrm{keV}$. This gives a stored energy $(E)$ of

$E_{e}=V_{p} n(3 / 2) k T=7.2 \times 10^{4} \mathrm{~J}$,

$E_{i}=3.6 \times 10^{4} \mathrm{~J}$,

where the total number of each species $\left(N=V_{p} n\right)$ is $5 \times 10^{19}$. As in EBT-I, a negative ambipolar potential which is consistent with collisionless electron confinement is assumed. Using the neoclassical collisioniess electron confinement time $\left(\tau=A^{2} \tau_{90}\right)$ and an effective aspect ratio of 40 results in a confinement time of about $0.2 \mathrm{sec}$ and an $\mathrm{n}_{\mathrm{\tau}}$ the order of $10^{13} \mathrm{sec} \mathrm{cm}^{-3}$. Under these conditions, approximately $500 \mathrm{~kW}$ of ECH bulk heating will supply the power lost by neoclassical processes, and the particle loss rate from the plasma is on the order of $2.5 \times 10^{20} \mathrm{sec}^{-1}$.

Taking $n=10^{14} \mathrm{~cm}^{-3}$ and $T_{i}=T_{e}=6 \mathrm{keV}$ as goals for the EBT-II Phase 2 plasma with neutral beam heating, the stured eneryies and particle numbers increase to 
ORNL/DWG/ $/ C D-70-499$

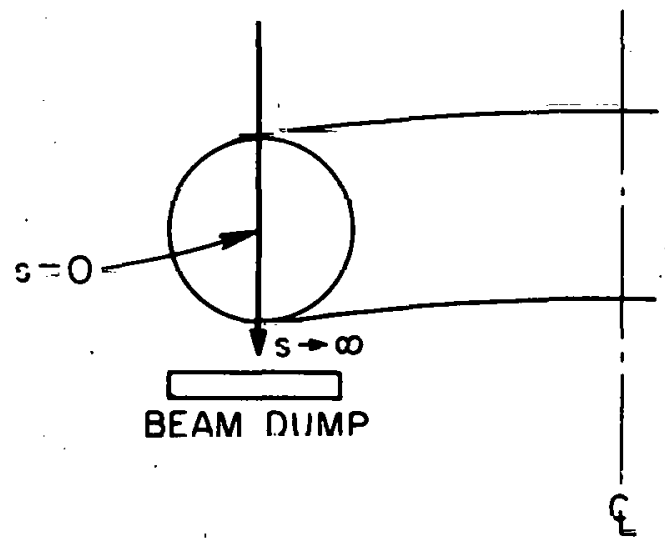

Fig. D.1. Side view of torus showing vertical injection geometry. Distance along injection path denoted by $s$. 
$E_{e}=E_{i}=1.4 \times 10^{5} \mathrm{~J}$

and

$N=1.0 \times 10^{20}$,

giving an increase of

$\Delta E_{\text {total }}=1.7 \times 10^{5} \mathrm{~J}$

and

$\Delta N=5 \times 10^{19}$.

Collisionless ion confinement with the separate ion heat source could allow values of nt to approach $10^{14} \mathrm{sec} \mathrm{cm}^{-3}$. Since this confinement depends on a positive ambipolar potential, it will probably be necessary to control both ion (neutral beam) and electron (bulk ECH) heating to achieve an equilibrium. Primitive arguments indicate that ion heat must be controlled around a $T_{j}$ set point and that the ECH must almost be turned off. (of course, detailed transport calculations must be carried out for these conditions, and development of the model codes will be accomplished during the near future.) Thus, in the injected phase of EBT-II, neutral beams need to be capable of supplying a net power to the plasma of approximately $300 \mathrm{~kW}$ in order to reach $\mathrm{n \tau} \approx 10^{14} \mathrm{sec} \mathrm{cm}^{3}$.

\section{D.2 TRANSFER OF NEUTRAL BEAM ENERGY TO THE PLASMA}

Of critical importance in choosing the accelerating potential for a neutral beam injection system is how the choice affects the energy transfer from the beam to the plasma. The first requirement, for obvious beam efficiency considerations, is that a reasonable fraction of the beam particles be ionized or charge exchange trapped on passing through the plasma. The rate at which the beam particles are ionized along the beam path can be obtained from a conventional calculation (e.g., see Ref. 2).

The fraction of particles not removed from the beam (as well as the radial distribution of the ionized beam particles) depends on the plasma radial density profile and the combined cross sections for charge exchange and electron and ion impact ionization. Figure 0.2 shows the individual and combined ettective cross sections $<\sigma_{c x} v>/ v,\left\langle\sigma_{i} v>/ v\right.$, and $\left\langle\sigma_{e} v>/ v\right.$ vs beam energy 3,4 tor Maxwellian 1 on and electron distributions of $0,3,6$, and $10 \mathrm{keV}$. Figure 0.3 shows the normalized radial heat deposition profile for several choices of central mean free path $\left[\lambda_{0}=\left(\text { no }_{\text {total }}\right)^{-1}\right]$ and for the two cases of parabolic and constant radial plasma density profiles. The curves of Fig. D. 3 are labeled with values of $\lambda_{0} / a$, where $a$ is the plasma minor radius. The fraction of particles not removed from an incident beam of small cross-sectional area passing through the center of the plasma is exp $\left(-2 a \lambda_{0}\right)$ for a constant radial plasma density and exp $\left(-4 a / 3 \lambda_{0}\right)$ for a parabolic profile.

The incident neutral beam power required to achieve a level of $300 \mathrm{~kW}$ removed from the beams by the plasma and the beam particle current trapped in the plasma are plotted vs beam particle energy in Fig. D.4. From these curves it can be seen that a beam energy near $20 \mathrm{keV}$ is a good choice for optimizing beam efficiency and particle loading of the vacuum system while still keeping a favorable energy deposition profile.

Detailed kinetic theory calculations remain to be done to determine the equilibration of the energy between the trapped beam particles and the background plasma and the losses due to charge exchange by 


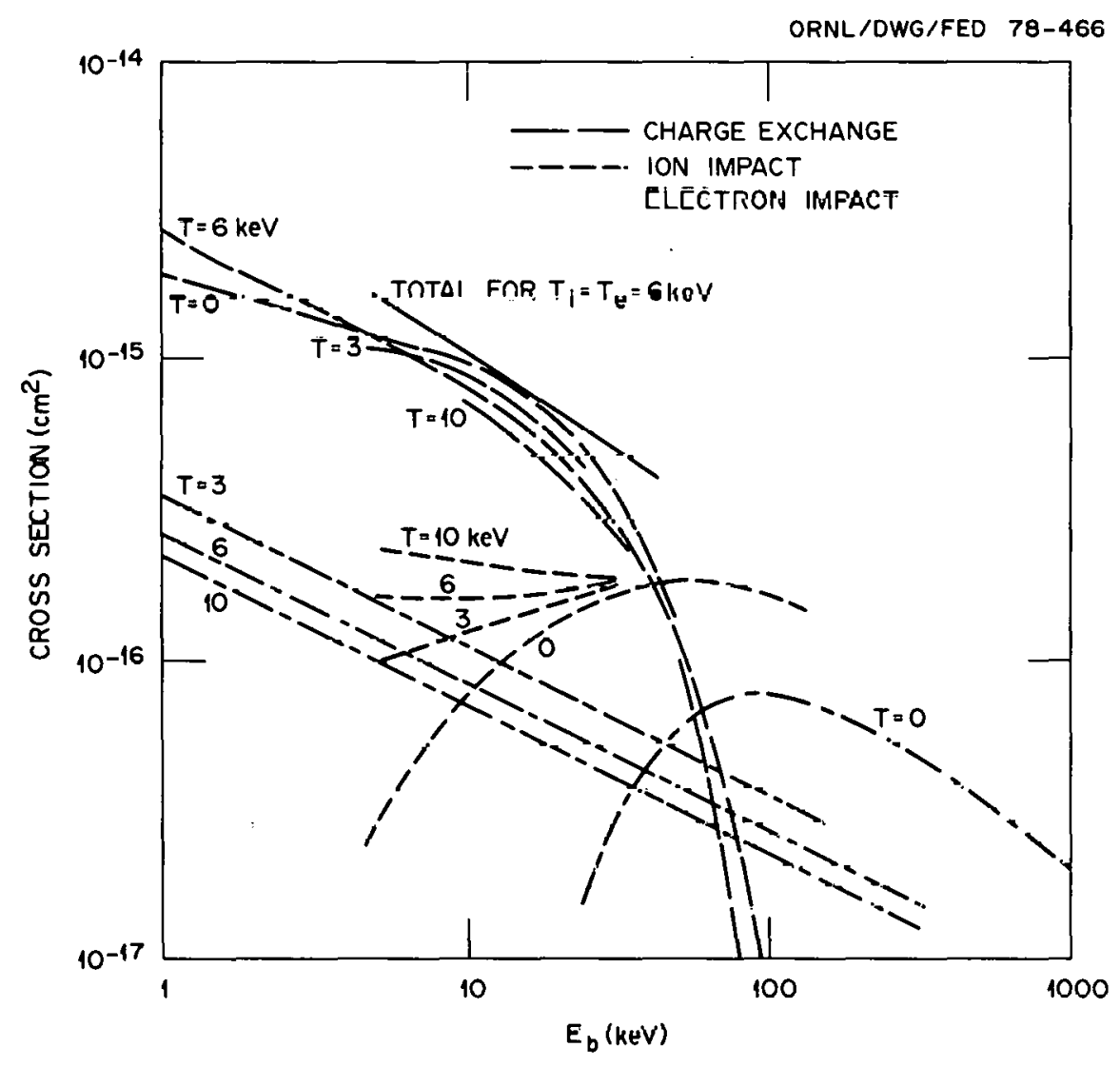

Fig. D.2. Separated and total cross sections for initial collision of injected neutral beam vs energy (Refs. 3 and 4). Maxwellian distributions assumed for target plasmas. 


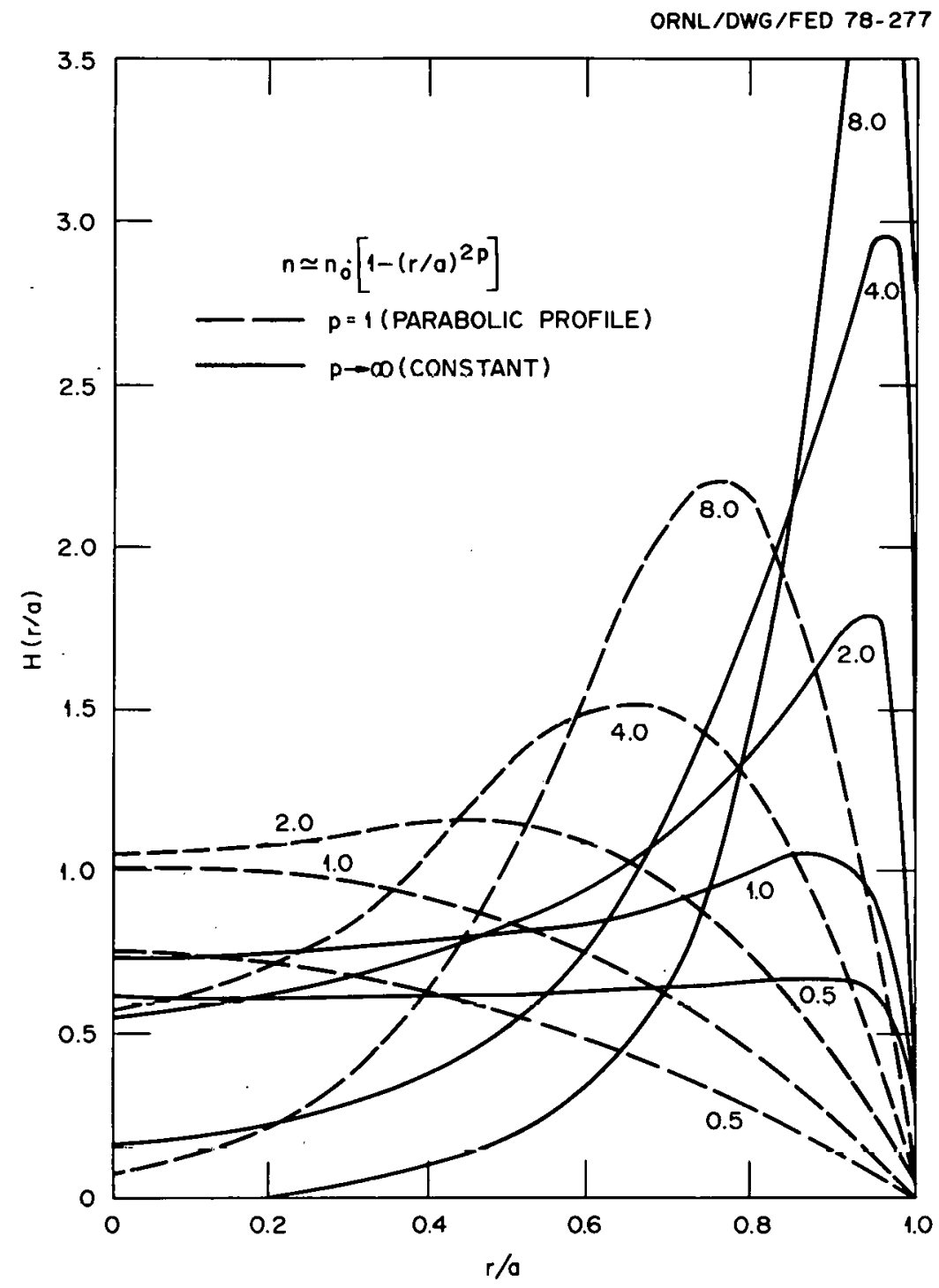

Fig. D.3. Fast ion deposition, $H(r / a)$. Curves are labeled with $a / \lambda_{0}$. Dashed curves are for parabolic density profile, and solid curves are for constant densily pruflle. 


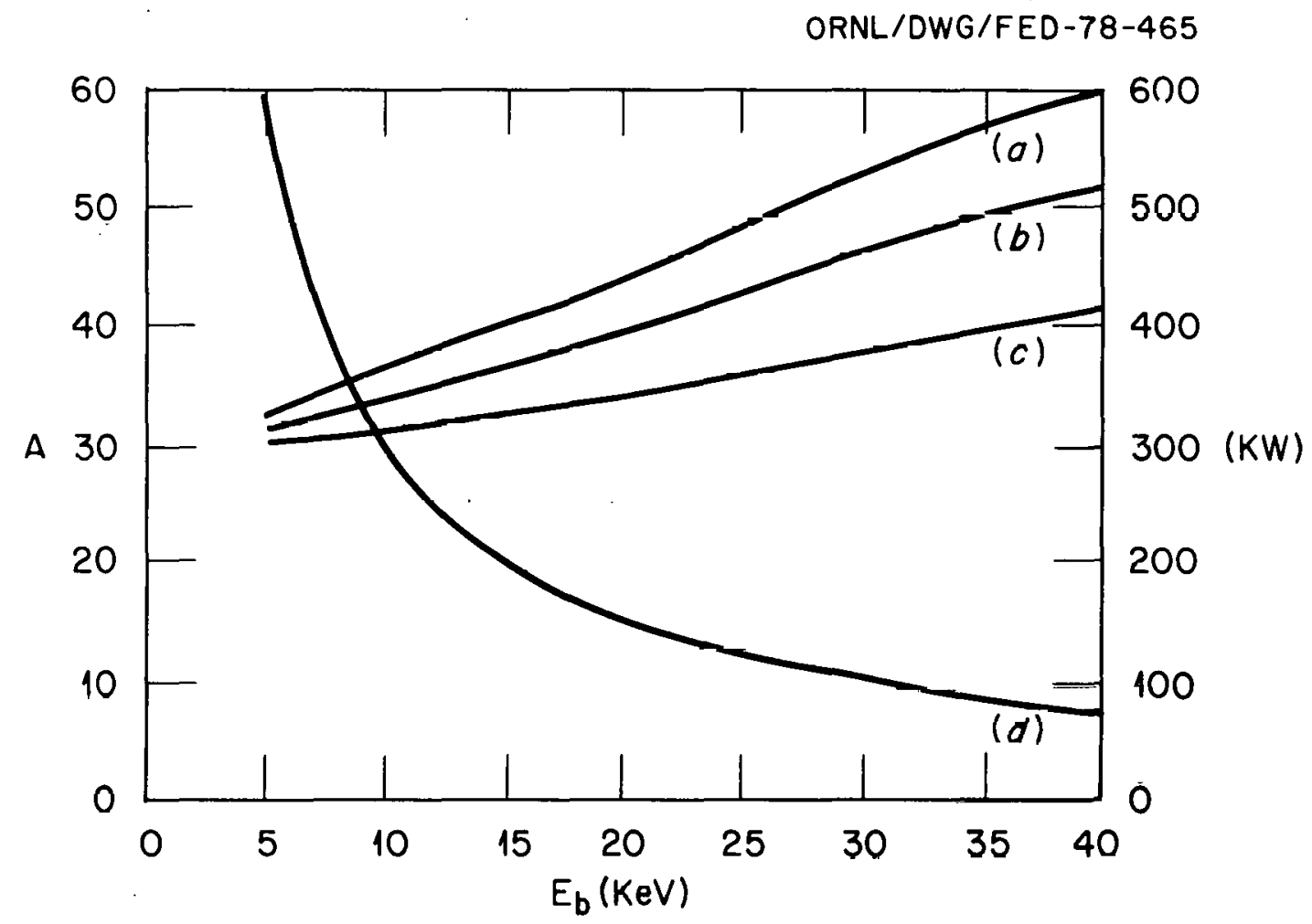

Fig. D.4. Beam power and current required to deposit $300 \mathrm{~kW}$ in the plasma vs beam enorgyi (a) bcam diamctcr - 0.5 plasma diameler, cyl Indrical beam, parabolic density; (b) pencil beam, parabolic density; (c) pencil beam, constant density; (d) current to plasma. 
the fast ions. This is important since the neutral currents required at $20 \mathrm{keV}$ in order to deliver the power to replace the plasma losses indicate that the fast ion population is a significant fraction of the total distribution. However, a meaningful kinetic calculation requires inclusion of the selfconsistent ambipolar electric field, which has not yet been done.

\section{3 VACUUM SYSTEM REQUIREMENTS FOR STEADY-STATE INJECTION}

The particle current from the beam lines represents a load on the vacuum system which must be continuously removed in a steady-state mode of operation. The power supplied by the beams must also be absorbed by the walls continuously. Let $I_{B}$ be the total injected particle current, $V_{B}$ the beam particle energy, and $K$ the fraction of the particles that goes into the toroidal plasma. From the discussion in Sect. D.1, the product $K_{B} V_{B}$ should be approximately $300 \mathrm{~kW}$. If this power were uniformly absorbed by the approximately $4.4 \times 10^{5}-\mathrm{cm}^{2}$ surface area of the EBT-II device, then the power density would be only $0.7 \mathrm{~W} / \mathrm{cm}^{2}$. Allowing a peak-to-average power a factor of ten higher leads to an estimated peak wall loading of $7 \mathrm{~W} / \mathrm{cm}^{2}$ from the toroidal plasma. On the other hand, the fraction of beam power not entering the toroidal plasma $(1-K) I_{B} V_{B}$, will be dissipated in the sectors where beam lines are located. Most of this power will go directly into the beam dump, but a portion of it will be dissipated by charge exchange neutrals resulting from collisions between trapped beam particles and background neutrals. These particles should strike the walls of the sectors containing beam lines in a band centered about the midplane with a width on the same order as the injected beam (this would be an area of approximately $2600 \mathrm{~cm}^{2}$ for each beam line). An accurate calculation of the power lost from the beams by charge exchange before being transferred to the toroidal plasma can be obtained from a kinetic theory calculation including a self-consistent distribution of the neutral density. However, a wall loading of $7 \mathrm{~W} / \mathrm{cm}^{2}$ would allow $18 \mathrm{~kW}$ per beam line (or a total of $72 \mathrm{~kW}$ for an injection system of four beam lines) to be dissipated by charge exchange, which is $25 \%$ of the total required beam power of $300 \mathrm{~kW}$. Thus one would not expect the peak wall loading to be liuch above $7 \mathrm{~W} / \mathrm{cm}^{2}$.

The particles in the untrapped fraction of the beam will be a minor perturbation on the particle balance of the toroidal plasma. Most of these particles will go directly into the beam dump. of the particles scattered to the walls in the midplane by charge exchange losses, a sizeable fraction will go directly into the beam dump, as well as into the beam input port and the sector vacuum port. Those that hit the cavity wall will cause the neutral pressure at the walls in the sectors having beam lines to be slightly higher than in the other seciors.

The particle and energy balançe in the toroidal plasma deserves a closer look. Let $\tau_{p}$ be the particle confinement time for ions and electrons in the toroidal plasma and $\tau_{h}$ be the ion energy confinement time $\left(\tau_{h}<\tau_{p}\right.$ due to charge exchange losses). Let a be the fraction of electron energy supplied by beam heating and $\underline{b}$ be the fraction of ion energy supplied by beam heating. Then

$K I_{B} V_{B}=\underline{a}\left(N k T_{e} / \tau_{p}\right)+\underline{b}\left(N k T_{i} / \tau_{h}\right)$.

If $V_{x}$ is the mean energy of particles in the charge exchange current $\left(j_{x}\right)$ out of the plasma and $W_{R}$ represents the power lost by radiation, then

$\left(N k T_{i} / \tau_{h}\right)=\left(N k T_{j} / \tau_{p}\right)+V_{x} j_{x}+W_{R}$

If $j_{0}$ is the particle current due to cold gas feed and $j_{r}$ is the particle current reflux from the walls to the plasma, then the particle balance equation is 
$K I_{B}+j_{0}+j_{r}=j_{x}+N / \tau_{p}$

This equation can be combined with the energy balance equation to eliminate $I_{B}$ *

$j_{0}+j_{r}=j_{x}+\left(N / \tau_{p}\right)\left(1-\underline{a k} T_{e} / V_{B}-\underline{b k} T_{i} \tau_{p} / V_{B} \tau_{h}\right)$.

If the same neutral pressure at the walls is maintained with and without beam heating, then $j_{r}$ is constant as a and $\underline{b}$ vary from 0 to 1 . In equilibrium, $j_{0}$ equals the current to the toroidal vacuum system in the absence of beams $(\underline{a}=\underline{b}=0$ ). A vacuum system with a pumping speed of $200,0001 \mathrm{iter} / \mathrm{sec}$ at $300 \mathrm{~K}$ has $j_{0}=10 \mathrm{~A}$ for a neutral pressure of $10^{-5}$ torr. For a neutral beam current of $10 \mathrm{~A}$ to deliver $300 \mathrm{~kW}$, the beam energy must be $30 \mathrm{keV}$. However, pumping speed will be somewhat higher due to the increased conductance from a fraction of the energetic particles lost by the plasma gning directly into the vacuum ports. If heating improves confinement as expected, so that $K I_{B} V_{B}$ ran he rediured tn undor $200 \mathrm{~kW}$ in stcady statc opcration, then the neutial piessuie call be Illdilldillil al: 1ess than 10-5 Eorr tör $V_{B}=20 \mathrm{keV}$.

\section{D.4 BEAM SYSTEM SPECIFICATIONS}

The information concerning the plasma and neutral beam characteristics and particle and energy balances presented in the previous sections must be combined in order to determine specifications for a neutral beam injection system for EBT-II. Further, some mention needs to be made of problems to be faced when integrating the beam system into the EBT-II system.

First, the choice of beam energy will be a compromise between the desires to keep total efficiency high, equipment simple, and particle loading of the vacuum system low and the desire to provide sufficient ion heating power. For the sake of ion heating efficiency, the beam energy should be kept low (below about $20 \mathrm{keV}$ ). On the other hand, particle balance considerations make a high beam energy desirable. Since some fraction of the particles in the neutral beam will have energies of one-half and one-third the accelerating potential, a value near 20 keV should be a satisfactonry rnmprọmise for a curceptual design.

Given the value of $20 \mathrm{keV}$ for the accclcriting potential, the leijuirud budll current can be dctcr ini ried. With $n_{\tau} \approx 10^{14} \mathrm{sec} / \mathrm{cm}^{3}$ and a confinement time estimate of about $1 \mathrm{sec}$, the power required to maintain the plasma equilibrium is about $300 \mathrm{~kW}$, not counting charge exchange losses from the beam before equilibration. (In order to heat the plasma to the final state, $1.7 \times 10^{5} \mathrm{~J}$ must be added. This would be supplied in about $0.5 \mathrm{sec}$ at a power of $300 \mathrm{~kW}$. Thus $300 \mathrm{~kW}$ is adequate for the transition perlod.) In order to make an allowance for beam particles not initially stopped in the plasma as well as fast ion charge exchange losses and other nondiffusive losses, an incident power of $500-750 \mathrm{~kW}$ should be available from the injection system.

Tlie loadiny of the vacuuril system by beam particlès has been discussed in Sect. D.3. In addition, the beam dumps should be capable of stopping all of the incident beam to allow the use of beam heating with target plasmas of lower density.

A dynamic control system will be needed for the neutral beam currents and neutral gas feeds and should also include regulation of microwave bulk ECH power. Because of the relation of energy confinement time to temperature and density, a constant level nf neutral beam heating above a critical lcvcl could cause thermal runaway. It must be possible to reduce the beam current as the confinement time is raised during the transition from the initial plasma state to the final equilibrium. The logical system response time should be on the order of the energy confinement time. Since proportional control of the beam current may be difficult, beam power could be regulated by a pulse modulation at a repetition rate 
which is fast compared to the confinement time. The feed of cold gas must also be adjusted to match the changing particle loads from the beams while keeping the vacuum system pumping speed fixed. Adjustments to microwave ECH bulk heating power will be needed simultaneously to keep the electron and ion temperatures in the desired balance, particularly since the electron density will be doubled along with the ion density. Presumably all these features can be included in a control system for EBT-II.

The total injected beam power can be supplied from several separate beam lines, the actual number to be determined by operational and engineering considerations. It would appear unwise to use either extreme of a single beam line or one per mirror section. Thus, as a starting point for further investigation, four beam lines were selected at $20 \mathrm{keV}$ and $12.5 \mathrm{~A}$ maximum $\mathrm{cW}$ each, for a maximum of $1 \mathrm{MW}$ extracted from the source.

In conclusion, the factors influencing the specifications for neutral beam injectors for EBT-II have been explored. The injection system should utilize multiple beam lines with dynamic control to provide hundreds of kilowatts at energies in the 20-keV range. The detailed current and voltage specification for each beam line must be fixed by engineering considerations, using readily available technology.

\section{REFERENCES}

1. Appendix $A$, this report.

2. J. A. Rome, J. D. Callen, and J. F. Clarke, Nucl. Fusion 14, 141 (1975).

3. D. R. Sweetman, Nucl. Fusion 13, 157 (1973), Fig. 10.

4. A. C. Riviere, Nucl. Fusion 11, 363 (1971). 
THIS PAGE

\section{WAS INTENTIONALLY LEFT BLANK}


APPENDIX E

RELEVANCE OF THE EBT-II EXPERIMENT TO AN EBT REACTOR 
THIS PAGE

WAS INTENTIONALLY

LEFT BLANK 
A number of features of the ELMO Bumpy Torus (EBT) confinement concept make it an attractive alternative approach for fusion power. The next-generation EBT device, EBT-II, could greatly facilitate an understanding of the physics and technology of fusion reactors, as well as provide the necessary inputs for determination of EBT's fusion economic relevance.

From the viewpoint of a reactor designer, an ideal fusion reactor would have the following characteristics: (1) steady-state operation, (2) moderate to high beta, (3) a relatively large aspect ratio, (4) modular construction, (5) a reasonable surface-to-volume ratio, (6) an operating wall loading of few megawatts per square meter, and (7) relatively straightforward assembly and maintenance. The EBT concept is the only approach under active study which seems at this time to satisfy all of these requirements. Because of these potential advantages of EBT, a uniform and self-consistent treatment of EBT reactor (EBTR) scaling and design has been studied and a reference design has been selected. 1 There are many uncertainties involved in extrapolating present plasma physics knowledge to predict how future devices will behave, but it is necessary to do so if the program is to stay on schedule. It is also clear that the more experimentally verified information available, the more credible the EBTR design will become. In this appendix several ways in which the EBT-II device will be relevant to EBTR are analyzed. Where quantitative information is required, the parameters in Table E.1 are used.l

Table E.1. EBT reactor reference parameters

\begin{tabular}{|c|c|c|}
\hline & $E B T R-48^{a}$ & EBTR-24 $4^{b}$ \\
\hline Plasma radius, a & $1.0 \mathrm{~m}$ & $1.0 \mathrm{~m}$ \\
\hline Aspect ratio, $A$ & 60 & 30 \\
\hline Major radius, $R_{0}$ & $60 \mathrm{~m}$ & $30 \mathrm{~m}$ \\
\hline Mirror ratio, M & 1.78 & 1.78 \\
\hline Ion temperature, $T_{i}$ & $15 \mathrm{keV}$ & $15 \mathrm{keV}$ \\
\hline Ion density, $N_{j} \times 10^{-20}$ & $1.2 \mathrm{~m}^{-3}$ & $1.25 \mathrm{~m}^{-3} \quad 2.13 \mathrm{~m}^{-3}$ \\
\hline Beta, $B(\%)$ & 25 & $24 \quad 42$ \\
\hline Magnetic field on axis, $B_{T}$ & $2.5-4.5 \mathrm{~T}$ & $2.5-4.5 \mathrm{~T}$ \\
\hline Number of coils, $\mathrm{N}$ & 48 & 24 \\
\hline Power, $\mathrm{P}_{T H}$ & $4000 \mathrm{MW}$ & $1775 \mathrm{MW} \quad 5300 \mathrm{MW}$ \\
\hline Power density, $\mathrm{P}_{T H} / \mathrm{V}_{\mathrm{p}}$ & $3.37 \mathrm{MW} / \mathrm{m}^{3}$ & $3.0 \mathrm{MW} / \mathrm{m}^{3} \quad 9.0 \mathrm{MW} / \mathrm{m}^{3}$ \\
\hline Neutron wall loạding, 'w & $1.1 .3 \mathrm{MW} / \mathrm{m}^{2}$ & $1 \mathrm{MW} / \mathrm{m}^{2} \quad 3 \mathrm{MW} / \mathrm{m}^{2}$ \\
\hline Cold zone, $\delta$ & $0.2 \mathrm{~m}$ & $0.2 \mathrm{~m}$ \\
\hline Blanket and shield thickness, $t_{s b}$ & $1.75 \mathrm{~m}$ & $1.75 \mathrm{~m}$ \\
\hline Coil inner radius, $r_{c}$ & $2.95 \mathrm{~m}$ & $2.95 \mathrm{~m}$ \\
\hline Current density, $\mathrm{J}_{\mathrm{c}}$ & $1500 \mathrm{~A} / \mathrm{cm}^{2}$ & $1500 \mathrm{~A} / \mathrm{cm}^{2}$ \\
\hline Coil radial thickness, $t_{c}$ & $0.71 \mathrm{~m}$ & $0.71 \mathrm{~m}$ \\
\hline Coil half lengțh, $L / 2$ & $1.30 \mathrm{~m}$ & $1.30 \mathrm{~m}$ \\
\hline
\end{tabular}

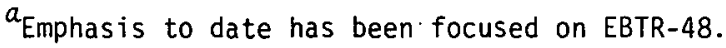

${ }^{b}$ Alternative smaller reactors with increased neutron wall loadiny are presenlly under study. 


\section{E.1. PROPERTIES OF THE EBT REACTOR}

The principal advantages of an EBTR stem from high beta, steady-state operation. The EBT-I experimental results have shown the confinement properties of the EBT concept to be very promising in this regard. EBT-II will provide the first test of the scaling laws in which the parameters are given a large increase; it thus represents a key step and decision point for the EBT program.

A reactor design and the required research and technology development program have resulted from the EBT Reactor System Study, which was undertaken in. early 1976. A multidisciplinary, self-consistent treatment of EBTR scaling and design led to the reference design (see Table E.l for parameters). The most important considerations in the plasma engineering, plasma physics, magnetics, neutronics, and design engineering areas are given in the design report. ${ }^{1}$ This design is based on a presently acceptable plasma model and relatively conservative engineering parameters, i.e., $21-2 \mathrm{MW} / \mathrm{m}^{2}$ of neutron wall loading and a 7.3-T maximum toroidal field. It is a steady-state, ignited system, heated by microwaves, neutral beams, or a combination of the two, with high power density and a large aspect ratio.

The EBT reactor described in this appendix is based on a specific set of assumed technological directions, design approaches, and plasma characteristics. There is no unique set of solutions to such design problems - the "optimum design" is strongly dependent on the weight given to the technological, engineering, and plasma physics constraints. For EBT-based systems, a small number of design choices is sufficient to indicate the major aspects of the final design: the choice of wall loading, mirror ratio, and limiting plasma pressure $(B)$ determines the size of a standard module of the system and, given total plant output, the number of modules in the system.

Problems of accessibility, structural design, and remote maintenance are considerably eased because of the large aspect ratio in EBTR and the fact that there are no pulsed fields. Several key features of the reactor study, the basic physics and technology assumptions relevant to the study, and its relevance to the proposed EBT-11 are discussed.

\section{E.1.1 POWER DENSITY SCALING}

The power density which dictates the desirable characteristics for the plasma in a deuteriumtritium ( $D$ T) burning system is given by

$\frac{P_{T H}}{V}=n_{i}^{2} \frac{\langle\sigma V\rangle}{4} Q_{F} \propto B^{2} B^{4} \frac{\langle\sigma V\rangle}{T^{2}}$,

where $P_{T H}$ is the thermonuclear power produced by the plasma, $V$ is the plasma volume, and $Q_{F}=17.6 \mathrm{MeV}$. In a low beta plasma, beta is limited to a few percent [it may be as large as $5-10 \%$ in a flux conserving tokamak $\left.(F C T)^{2}\right]$, and $\left.\angle \sigma V\right\rangle / T^{2}$ exhibits a broad maximum between 10 and $20 \mathrm{keV}$, while the magnetic field, $B$, is limited by the technological constraints; an optimum operating point which is consistent with the overall plant can be chosen within these criteria. In a high beta system, additional tradeoffs are involved: for example, operation outside the range which maximizes $\langle\sigma v\rangle / T^{2}$ might become desirable to keep density, $n$, within limits because of microwave cutoff or neutral beam penetration requirements. The tradeoffs involved in the optimization of the high beta cycle are unique and are formulated for the study. ${ }^{1}$ The tradeoffs between beta and $B$ have been considered and high beta and low B (the minimum field strength assumed feasible is $225 \mathrm{kG}$ ) operation is found to be desirable. Figure E.1 shows the relationship between plasma, magnetic field, and power production parameters. 


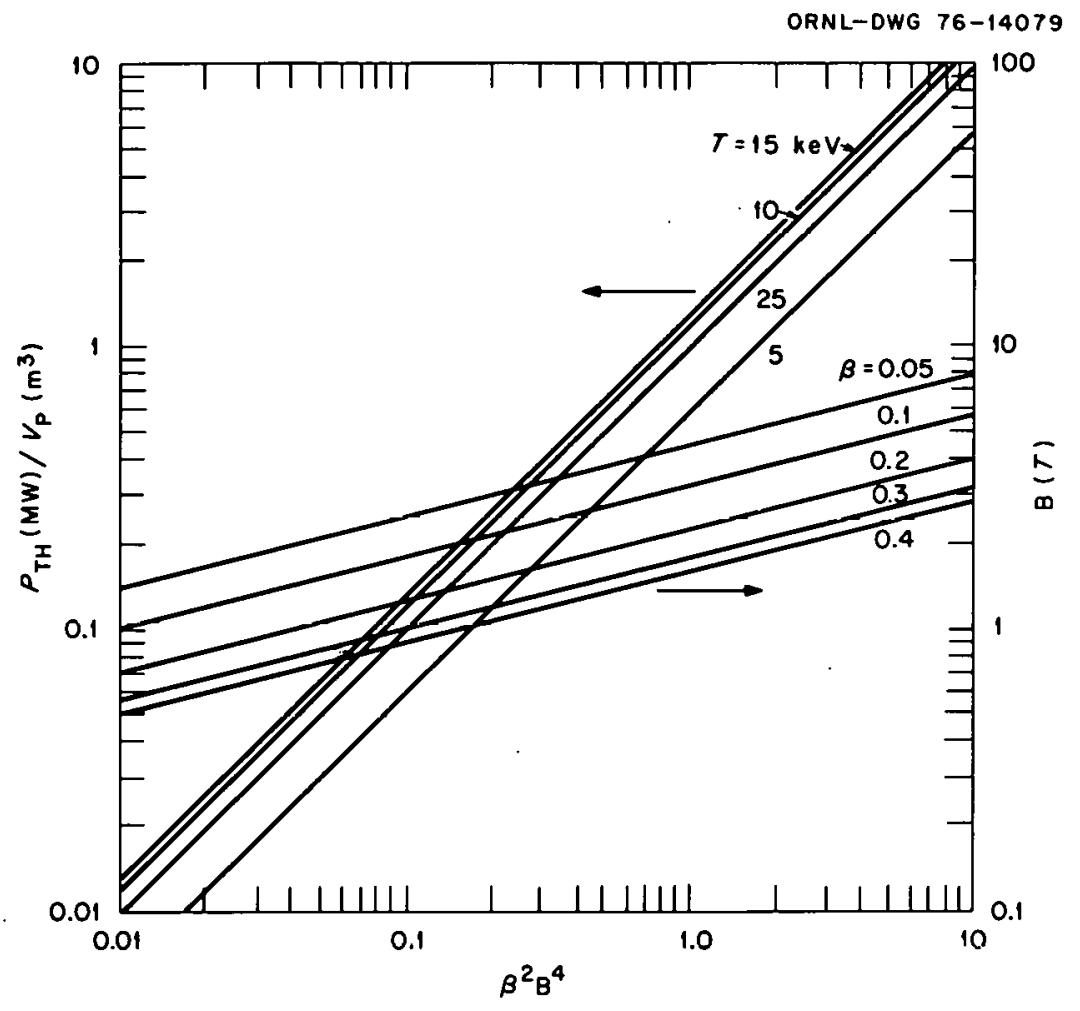

Fig. E.1. Relationship between plasma, magnetic field, and power production parameters. 


\section{E.1.2 STARTUP PROCEDURES}

Startup scenarios consistent with microwave cutoff $(120 \mathrm{GHz})$ or neutral beam penetration (150 keV) requirements have been derived to take advantage of high beta operation in EBT. At $T=15 \mathrm{keV}$, a beta of $\leqslant 0.35$ is required for $120-\mathrm{GHz}$ microwave penetration. Similarly, neutral beam attenuation, which is proportional to exp $(-n \sigma x)$, also places a restriction on maximum density if hollow density profiles are to be avoided. The dynamics of the EBTR system have been analyzed to establish procedures for achieving a high beta operating point at acceptable density, consistent with constraints imposed by other reactor support systems. Flexibility is retained in the analysis to permit any physically acceptable departures from an assumed scaling argument. The ultimate requirements will be determined by EBT-II experiments, which will either validate the present assumptions or provide the basis for change.

The power requirements for startup and recirculating power for auxiliary functions and plasma sustenance will dictate power supply criteria and demand from the grid. Two possible techniques have been suggested to minimize the power required for startup. 3 The low density startup relies on plasma losses scaling as $n^{2}$, and the restricted cross section or large aspect ratio (A) procedure depends on $\tau \propto A^{2}$ during startup. Detailed analysis of these possible startup scenarios, means of controlling density and restricting the cross section, and the basic plasma stability during the processes are under consideration.

\section{E.1.3 MAINTAINING STEADY STATE}

Fueling of the EBT reactor is required to take advantage of steady-state operation; however, this requirement is not unique since it applies to all large systems where the burn time will exceed the particle containment time. In EBT-II, particle density will be maintained by introducing neutral gas into the chamber. The EBTR plasma is too large ( $\sim 1 \mathrm{~m}$ radius) to permit adequate neutral penetration for this technique to be effective, so that the particle density will be maintained by some other means, such as pellet rueling.

A method for removing residues (fusion products) and impurities may be necessary if the particle dynamics of transport processes do not lead to this result naturally. In EBT-I, impurities do not accumulate in the central region of the plasma due to the short confinement of impurities in the surface plasma. EBT-II experiments will either validate EBT-I results or will show that the EBTR will require residue removal and impurity control mechanisms.

Power requirements for sustaining the stabilizing annuli need to be examined in detail, although preliminary calculations show that this is not a problem because the ring plasma occupies a relatively small volume. The recirculating power fraction is discussed in Sect. E.1.4.

\section{E.1.4 RECIRCULATING POWER FRACTION}

A simple energy balance which accounts for the circulating power in the system defines a figure of merit for the reactor. An upper limit for the recirculating power fraction (defined as the recirculating power divided by the power produced), which is commonly used as a performance indicator, is specified by a cost breakdown of the reactor. Some of the recirculating power is used for auxiliary functions such as magnet refrigeration and blanket cooling, while the rest is available to be fed back into the plasma in order to sustain the plasma conditions. Since the auxiliary power requirements can be relatively well defined and are almost independent of plasma physics assumptions (for example, for the EBTR reference design they are less than $0.2 \%$ of the power produced), the upper 1 imit on the recirculating power fraction effectively restricts the amount of power available for feedback into the plasma, and hence demands that certain plasma conditions be fulfilled. 
In the simplest case, the sustenance power is considered to be that necessary to drive the stabilizing annuli and to fuel the central zone of the plasma to maintain the plasma density during steady-state operation. The burning portion of the plasma is assumed to be fueled with a minimum expenditure of energy (say, by pellet fueling); hence, the power necessary for the microwave sources to sustain the annuli becomes the determining factor for the performance indicator and a measure for overall reactor efficiency.

In steady state the annulus power balance is $P_{\mu A}=P_{T A}+P_{B A}+P_{C A}$, where $P_{\mu A}$ is the microwave power required to sustain the annulus, $P_{\tau A}$ is the power lost by thermal conduction and convection, $P_{B A}$ is the bremsstrahlung radiation power loss, and $P_{C A}$ is the power lost by synchrotron radiation. The emission of synchrotron radiation from the hot electron annulus in EBT and a large-SCale EBTR waS estimated in the initial EBT proposal. ${ }^{4}$ More detailed calculations have been carried out for the EBTR reference design and reported in Appendix $C$ of Ref. 1. In the latter work, the synchrotron emission amounted to a few tens of kilowatts per sector at worst compared to a few tens to a few hundreds of kilowatts of transport losses for the data chosen. It is important, however, that this subject be reviewed frequently as more information becomes available about the annuli (and their scaling laws) from the EBT-S experiments and proposed EBT-II experiments.

Although the calculations in Ref. I have yielded inconsequential emission levels, the results are sensitive to details of the hot electron distribution function. The Maxwellian distribution function treated in Appendix $C$ of Ref. l overestimates the Doppler broadening and therefore slightly overestimates emission levels, while it neglects correction factors associated with the monotonically decreasing, but non-Maxwellian, distribution in velocity perpendicular to the magnetic field. Distortions in velocity distribution might lead to changes of order unity. ${ }^{5}$ Finally, the emission level is sensitive to the annulus electron temperatures and would be very important for temperatures in excess of 1 MeV.

More information on the requirements for sustenance of annuli will be determined by EBT-II experiments.

\section{E. 1.5 OVERALL PLANT DESIGN}

The EBT reactor magnetics, design engineering, and neutronics areas have been studied and the design parameters have been chosen to utilize the level of development of existing technology or technology which is encompassed by the near-term goals of existing development programs. A general plan view of the entire EBTR plant is shown in Fig. E.2 and a brief description of the various reactor. subsystems is given below.

\section{E.1.5.1 Toroidal Magnet System Design}

Aside from the plasma constraints, the main goal was the design of coils which could be built with existing technology. Independent of plasma physics considerations, the number of coils, mirror ratio, coil radius, and the reactor major radius can all be related by the geometry of the vacuum magnetic fields. Plasma physics considerations further relate the allowable mirror ratios to the aspect ratio, electron ring beta, stable plasma volume, magnetic axis shift, and similar quantities.

The steady-state nature of an EBT makes it very reasonable to pursue a superconducting coil design rather than a normal copper coil design because of power efficiency. In contrast to tokamak reactors, EBT has no pulsed fields. Hence the more exotic conductor designs, such as multicomponent matrices (CuNi), a very fine superconductor filament size $(<10 \mu \mathrm{m})$, hollow or forced-flow conductors, cabling and braiding of composite conductors, etc., are not required. With respect to superconducting materials, the cheaper and more ductile NbTi is preferred, but Nh, Sn as an nntion is also considered. Since there arc no pulsed fields, eddy currents and associated diamagnetic forces are not severe problems. 


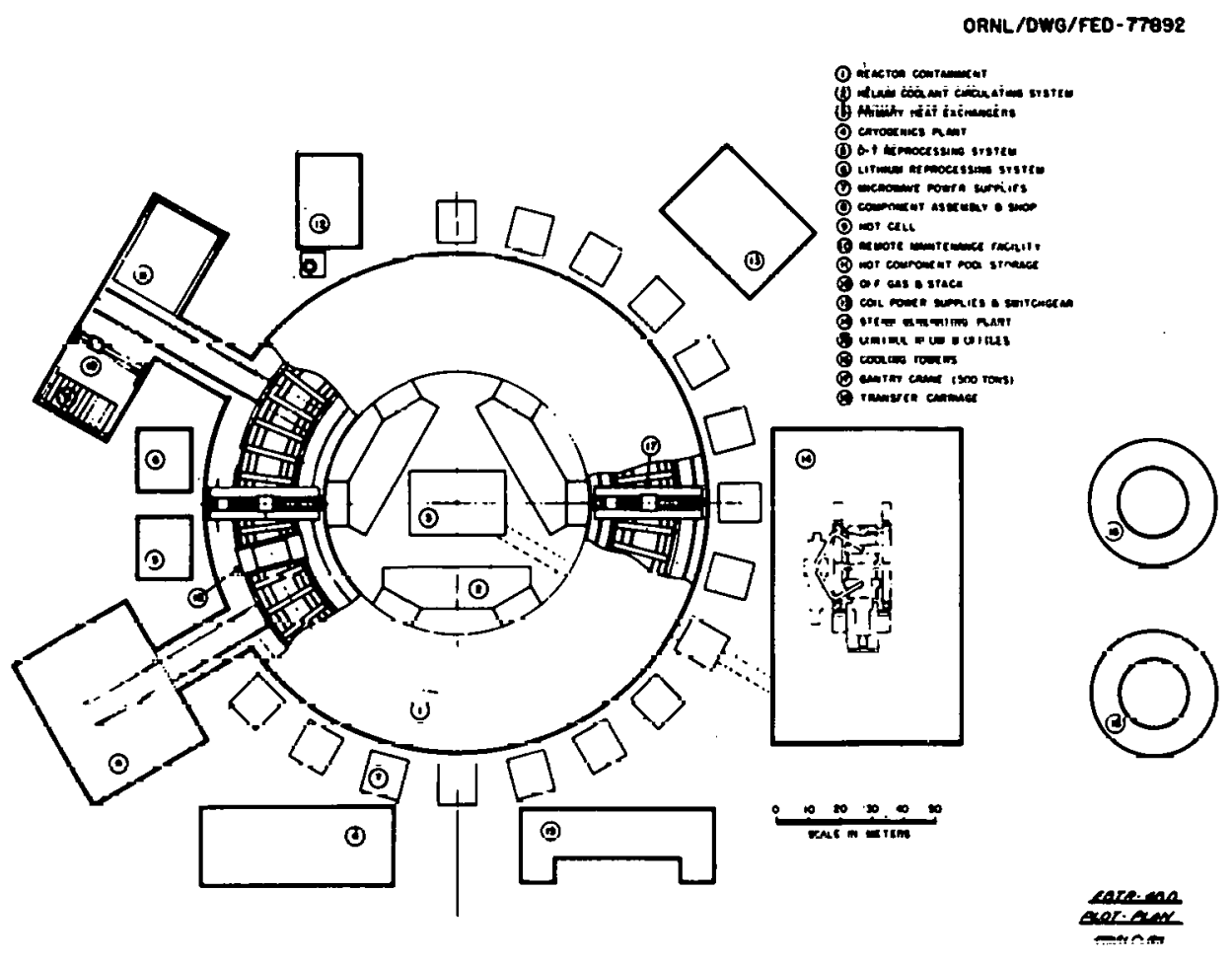

Fig. F.7. FRTR plann view. 
In contrast to the tokamak, the EBT has a large aspect ratio, so the magnetic field and loading are nearly symmetrical and there is no need to use D- or oval-shaped coils to minimize the bending moments. Circular coils are used. Natural convection pool-boiling is chosen as the cooling method, since the transient heat loads are minimal because of steady-state fields and adequate shielding space is available within moderate-size coils.

As long as the ratio of major radius to the number of coils is fixed, the field strength produced by modular magnets of the same size is not sensitive to the size of the reactor. The mirror ratio and coil bore $f i x$ the length of the blanket and shield modules, which are located between adjacent magnets. These parameters are fixed by plasma physics and blanket-shield engineering requirements. Thus, an important feature and advantage of an EBTR is that the magnets may be made modular and used in reactors of different sizes and power outputs.

The reactor design did not include aspect ratio enhancement (ARE) coils. These coils are of tremendous aid to a research device such as EBT-II because they allow direct tests of aspect ratio scaling in a single device and because they improve plasma confinement to that of a much larger device without ARE coils, but they do so at the expense of added complexity. In a reactor with blanket and shielding requirements, ARE coils would triple the number of superconducting magnets required (for a fixed number of sectors) and would greatly complicate assembly and maintenance. Therefore, the use of ARE coils is not contemplated for an EBTR.

\section{E.1.5.2 Mechanical Design}

Conceptual designs have been made of EBT reactors based on a modular concept with identical superconducting coils for a wide range of sizes (major radius ranging from a minimum of $30 \mathrm{~m}$ to a maximum of about $120 \mathrm{~m}$ ). The entire torus is enclosed in a concrete moat which provides structural support for the torus and biological shielding to reduce radiation levels to limits which are tolerable for personnel on a constant-exposure basis. All maintenance is accomplished from overhead. The necessary remote maintenance equipment is mounted on top of the moat and can be moved over the top of the torus. Power supplies for all the EBTR components, including the refrigeration systems for the cryogenic coils, and the tritium recovery processing systems are located outside the moat. The torus is constructed of modules to make assembly and remote maintenance less difficult.

The main torus consists of five regions, four of which are concentric: (1) plasma, (2) first wall, (3) blanket, and (4) shield. The fifth region, the coil structure aperture, has been shifted invard approximately $25 \mathrm{~cm}$ in order to increase the confinement properties of the system (see Appendix $B$ of Ref. 1). These five regions are continuous around the torus and are composed of cylindrical modules. The assembiy of the torus is straightforward. ${ }^{1}$

Heat removal is accomplished in three separate components of the torus: first wall, blanket, and shield. The nuclear performance of the first wall and blanket has been evaluated using a onedimensional analysis. The calculations showed that about $97 \%$ of the fusion power produced is deposited in there two components. While the hlanket. ahsorhs most of the energy produced by the plasma, some additional shielding is provided to protect the superconducting coils. This shield, which is a mixture of stainless steel, lead, and borated water, protects the magnets adequately and keeps the neutron damage and heating levels in the superconductors within tolerable limits.

One of the more difficult problems associated with low aspect ratio fusion reactors is that of maintenance because of their inherent inaccessibility. This particular problem is alleviated in the EBTR chiefly because of its large aspect ratio and the absence of a poloidal field system. All maintenance is accomplished using the overhead access described above. 
The flexibility afforded by the EBTR configuration leads to several sets of engineering approaches. ${ }^{1}$ The large aspect ratio, reduced forces, and lack of pulsed magnetic fields make the EBTR very attractive from the engineering point of view.

\section{E.1.5.3 Neutronics}

Preliminary neutronics analyses for the EBTR design have been carried out using the one-dimensional discrete ordinates code ANISN to assess the capability of a lithium-laden prototypic blanket configuration for recovering the kinetic energy of the fusion neutrons and secondary gamma rays in the form of heat and for breeding tritium. The effectiveness of the shield surrounding the blanket in reducing the radiation incident on the toroidal magnet coils was also determined by comparing the energy deposition rate and radiation damage in the coils as a function of the shield thickness. The following observations and conclusions are noted.

1. Ihe energy deposition rate in the blanket is high enough $(\leq 96 \%)$ to achieve useful extraction of heat for the generation of power.

2. A 50 - to $60-\mathrm{cm}$-thick shield is adequate and effective enough to reduce the radiation incident and energy deposition in the toroidal magnets.

3. The high tritium breeding ratio (1.35) provides enough latitude to allow the inclusion of required ports and penetrations and still achieve a fertile reactor.

\section{E.2. SEQUENCE OF EDT DEVICES}

Commercial feasibility of the EBT concept for power generation rlust be established through the application of current and near-term technologies, practical design approaches, and flexible plasma characteristics in order to demonstrate and ensure the concept's economic viability. In this section, the recommended three-phase plan following the operation of EBT-II is summarized and physics and technology issues relevant to these devices are outlined.

\section{E.2.1 EBT-T}

EBT-T is seen as the Technology/Test stage of EBT development in which the important reactor technology programs of systems startup, fueling, massive supplementary ion heating, impurity control, etc. will be tested. EBT-II is regarded as the first signifirant. srale-un physirs experiment, whereas the EBT-T device would be expected to demonstrate reactor-relevant technology and systems integration experience in hydrogen plasma. A conclusive demonstration of ignition would probably be deferred to the succeeding device since the materials handling problem from a D-T burn would preclude the flexibility desired at this stage. Although no design work has yet been done, there are various routes for EBT-T which could become operational in the mid-1980's with an accelerated program plan.

\section{E.2.2 EBT-IT}

EBT-IT should represent the first test of true controlled fusion in an EBT system sized and designed to cope with the radioactive materials and the remote handling necessary at this stage. It would be a true demonstration of the physics/engineering feasibility of an EBT reactor. Although no detailed design work has yet been initiated for EBT-IT, EBT-IT is expected to have magnetic field and microwave frequency requirements similar to EBT-II and EBT-T. The range of parameters given in Table E.2 is expected to be representative of EBT-IT. Also, EBT-IT is expected to form a complementary part 
Table E.2. Expected parameter range for EBT-IT

\begin{tabular}{ll}
\hline Plasma radius, a & $\sim 0.5 \mathrm{~m}$ \\
Aspect ratio, A & $\sim 20-30$ \\
Mirror ratio, M & $\sim 2$ \\
Magnetic field on axis, $\mathrm{B}_{\mathrm{T}}$ & $\sim 3-6 \mathrm{~T}$ \\
Temperatures, T & $\sim 8-12 \mathrm{keV}$ \\
Densities, $\mathrm{n}$ & $\sim 10^{20 \mathrm{~m}-3}$ \\
Beta, $B_{\text {toroidal }}$ & $\sim 10-20 \%$ \\
Neutron wall loading, $\mathrm{L}_{\mathrm{W}}$ & $\sim 0.5-1.0 \mathrm{MW} / \mathrm{m}^{2}$ \\
Total fusion power, $\mathrm{P}_{\mathrm{TH}}$ & $\sim 150-500 \mathrm{MW}$ \\
Power density, $\mathrm{P}_{\mathrm{TH}} / \mathrm{V}$ & $\sim 3.5-7 \mathrm{MW} / \mathrm{m}^{3}$ \\
Microwave frequency & \\
$\quad$ Bulk heating & $\sim 120 \mathrm{GHz}$ \\
$\quad$ Profile heating & $\sim 60-80 \mathrm{GHz}$ \\
Neutral beam energy $\left(D^{\circ}\right)$ & $\sim 50-80 \mathrm{keV}$ \\
Plasma scrapeoff region, $\Delta$ & $\sim 0.1-0.2 \mathrm{~m}$ \\
Shield thickness, $\Delta_{\mathrm{S}}$ & $\sim 0.4-0.5 \mathrm{~m}$ \\
Coil inner radius, $\mathrm{r}_{\mathrm{C}}$ & $\sim 1.2 \mathrm{~m}$ \\
Current density, $\mathrm{J}$ & $\sim 1500-1800 \mathrm{~A} / \mathrm{cm}^{3}$ \\
\hline
\end{tabular}

of the Department of Energy's overall magnetic fusion program by addressing, in collaboration with industry and other laboratories, issues which are not studied elsewhere and by reinforcing other areas which in the present program receive only limited attention. Following the successful operation of EBT-II and EBT-T, and given an accelerated program plan, EBT-IT could be operational in the late 1980 's.

\section{E.2.3 EBT-R}

EBT-R should represent the commercial prototype demonstration phase. Since the plasma physics requirements will be (or at least are expected to be) the same for the ignition device and the commercial prototype, they will reduce the time and cost required to proceed from ignition demonstration to commercial operation.

\section{E.3. RESEARCH, DEVELOPMENT, AND DEMONSTRATION NEEDS}

The research, development, and demonstration ( $R D \& D$ ) needs include the experimental and theoretical plasma physics studies, system studies, and millimeter microwave power source development. RD \& $D$ needs with regard to other technology and engineering areas are not discussed here, since aside from the central hot plasma, many EBT systems are similar to (though in many cases simpler than) the systems envisioned for tokamaks.

\section{E. 3.1 PLASMA PHYSICS NEEDS}

In order to establish the confinement properties of the EBT concept, research in plasma physics is required to lay a basis for understanding the known as well as the dimly perceived problems. Continued effort in plasma engineering is required to couple experimental and theoretical plasma physics understanding with the design of hardware for EBT-T, EBT-IT, and EBT-R (see Sect. E.2). 


\section{E.3.1.1 Experimental Activities}

It is necessary to augment the diagnostic capability to permit increasingly convenient and reliable measurements of the plasma properties. Experimental investigation in the following areas is particularly important.

1. Properties of toroidally confined ions and electrons.

2. Neutral atom and impurity distribution.

3. Annulus properties and stability.

4. Toroidal stability analysis.

5. Supplementary ion heating.

6. Startup.

7. Fueling.

\section{E.3.1.2 Theoretical Activities}

The following key issues require theoretical study.

1. Transport and scaling.

2. Microwave and neutral beam heating.

3. Equilibrium and particle orbits.

4. MHD stability.

5. Microscopic stability.

6. Stability of the annulus.

\section{E. 3.2 SYSTEM STUDIES}

Fusion power system design requires the application of techniques from many disciplines of physics and engineering. System studies involve the use of the results from applied physics analyses, both to design the plasma system itsel $\bar{f}$ and to establish the criteria needed to permit design engineering to proceed self-consistently. The design process of an EBTR is difficult because some of the most critical aspects of the system and its behavior are not well understood at present; thus, the procedure involves making plaușible estịmates and retaining flexibility so that the desian can always accommodate new results from theoretical and experimental programs listed earlier. The following are key issues.

1. System modeling, including plasma dynamics, fueling, size scaling, coupling of plasma and systems, etc.

2. Power balance.

3. System economics.

4. Tradeoff studies.

5. System integration.

\section{E.3.3 MICROWAVE DEVELOPMENT}

Multimegawatt, continuous wave $(\mathrm{CW})$, electron cyclotron heating (ECH) systems operating at $120 \mathrm{GHz}$ are required by the EBT-T, $-I T,-R$, and subsequent programs. This power generation capability is clearly beyond the present state of the art for microwave active devices (i.e., tubes). An intensive development program has been undertaken through subcontract with private industry.

Immediately following the development of EBT-II ECH systems utilizing an array of 200-kW, 120-GHz microwave power modules, system developments for EBT-T, $-I T$, and $-R$ will proceed. It is anticipated 
that these systems will consist of multiple arrays of power modules modeled by the EBT-II systems.

Since the anticipated requirements of the microwave heating systems in EBT-T, -IT, and - R mainly involve increased microwave power input, and since such needs can be met through the use of multiple power source arrays, it is expected that the required systems will be attainable in a timely manner following the successful operation of the EBT-II system.

\section{E.4. CONCLUSIONS}

The EBTR described here is based on a specific set of assumed plasma characteristics, near-term technological directions, and engineering approaches. No doubt there are several acceptable sets which ultimately lead to commercial fusion power, and the set described here should be viewed in this context. It appears that the uncertainties with regard to commercial feasibility of EBTR are associated with uncertainties in the plasma physics performance; there are no anticipated limitations in the areas of technology and engineering. With the proposed EBT-II experiments, the uncertainties in plasma physics performance should be resolved within the next four to five years, and a multiphase plan for demonstrating commercial feasibility of the EBT concept should be undertaken. A three-phase plan for carrying out such a demonstration has been outlined. It is emphasized that this is a recommended plan and not the adopted plan of the EBT Program.

\section{REFERENCES}

1. D. G. McAlees, N. A. Uckan, E. S. Bettis, C. L. Hedrick, E. F. Jaeger, D. B. Nelson, L. W. Owen, J. F. Roberts, R. T. Santoro, D. A. Spong, H. L. Watts, H. T. Yeh, L. M. Lidsky, D. A. Ehst, J. S. Herring, D. L. Kaplan, A. Pant, and R. E. Potok, The ELMO Bumpy Torus Reactor (EBTR) Reference Design, ORNL/TM-5669, Oak Ridge, Tennessee (November 1976).

2. J. F. Clarke, High Beta Flux-Conserving Tokamaks, ORNL/TM-5429, Oak Ridge, Tennessee (June 1976).

3. R. A. Dandl, R. A. Dory, H. O. Eason, G. E. Guest, E. G. Harris, G. R. Haste, C. L. Hedrick, H. Ikegami, E. F. Jaeger, N. H. Lazar, J. N. Luton, D. G. McAlees, D. H. McNeill, and D. B. Nelson, Research Program for Plasma Confinement and Heating in ELMO Bumpy Torus Devices, ORNL/TM-4941, Oak Ridge, Tennessee (June 1975).

4. R. A. Dandl, H. O. Eason, A. C. England, G. E. Guest, C. L. Hedrick, and J. C. Sprott, The ELMO Bumpy Torus Experiment, ORNL/TM-3694, Oak Ridge, Tennessee (November 1971).

5. G. Bekefi, Radiation Processes in Plasmas, John Wiley, New York, 1966. 
THIS PAGE

\section{WAS INTENTIONALLY \\ LEFT BLANK}


ORNL/TM-5955

Dist. Category UC-20 f, g

\section{INTERNAL DISTRIBUTION}

$\begin{array}{ll}\text { 1. } & \text { F. W. Baity, Jr. } \\ \text { 2. } & \text { C. F. Barnett } \\ \text { 3. } & \text { D. B. Batchelor } \\ \text { 4. } & \text { L. A. Berry } \\ \text { 5. } & \text { F. M. Bieniosek } \\ \text { 6. } & \text { J. D. Callen } \\ \text { 7. } & \text { J.A. Cobble } \\ \text { 8-70. } & \text { R. A. Dandl } \\ \text { 71. } & \text { R. A. Dory } \\ \text { 72. } & \text { H. O. Eason, Jr. } \\ \text { 73. } & \text { J. C. Glowienka } \\ \text { 74. } & \text { G. R. Haste } \\ \text { 75. } & \text { C. L. Hedrick, Jr. } \\ \text { 76. } & \text { M. E. Hesse } \\ \text { 77. } & \text { S. Hiroe } \\ \text { 78. } & \text { E. F. Jaeger } \\ \text { 79. G. G. Kelley } \\ \text { 80. } & \text { S. P. Kuo } \\ \text { 81. } & \text { N. H. Lazar }\end{array}$

\author{
82. J. N. Luton \\ 83. 0. B. Morgan, Jr. \\ 84. D. B. Nelson \\ 85. H. Postma \\ 86. B. H. Quon \\ 87. M. W. Rosenthat \\ 88. D. A. Spong \\ 89. N. A. Uckan \\ 90. T. Uckan \\ 91. T. L. White \\ 92-93. Laboratory Records Department \\ 94. Laboratory Records, ORNL-RC \\ 95. Y-12 Document Reference Section \\ 96-97. Central Research Library \\ 98. Fusion Energy Division Library \\ 99. Fusion Energy Division Communications \\ Center \\ 100. ORNL Patent Office
}

\section{EXTERNAL DISTRIBUTION}

101. D. J. Anthony, Energy Systems and Technology Division, General Electric Co., 1 River Rd., Bldg. 23, Rm. 290, Schenectady, NY 12345

102. W. B. Ard, McDonnell Douglas-East, P.0. Box 516, St. Louis, M0 63166

103. C. Baker, Fusion Engineering Department, General Atomic Co., P.0. Box 81608, San Diego, CA 92138

104. R. E. Balzhiser, Electric Power Research Institute, 3412 Hiliview Ave., P.0. Box 10412, Palo A1to, CA 94304

105. W. B. Briggs, McDonnel1 Douglas-East, P.0. Box 516, St. Louis, M0 63166

106. S. Buchsbaum, Research Communications Principles Division, Bell Telephone Laboratories, Inc., Murray Hill, NJ 07974

107. M. Clark, Combustion Engineering, Windsor, CT 06095

108. J. F. Clarke, Office of Fusion Energy (ETM), Mail Stop G-234, Department of Energy, Washington, DC 20545

109. F. H. Coensgen, Lawrence Livermore Laboratory, P.0. Box 808, Livermore, CA 94550

110. F. E. Coffman, Office of Fusion Energy (ETM), Mail Stop G-234, Department of Energy, Washington, DC 20545

111. P. L. Colestock, Plasma Physics Laboratory, Princeton University, P.0. Box 451, Princeton, NJ 08540

112. R. W. Conn, University of Wisconsin, Department of Nuclear Engineering, Madison, WI 53706

113. T. Consoli, Centre d'Etudes Nucleaires de Grenoble, B.P. 85 Centre de Tri, 38041 Grenoble Cedex, Grenoble, Frarice

114. E. C. Creutz, Bishop Museum, P.0. Box 6037, Honolulu, HI 96818

115. F. L. Culler, Electric Power Research Institute, 3412 Hillview Ave., P.0. Box 10412, Palo Alto, CA 94304

116. J. M. Dawson, Department of Physics, University of California, Los Angeles, CA 90024

117. S. 0. Dean, Office of Fusion Energy (ETM), Mail Stop G-234, Department of Energy, Washington, DC 20545

118. R. DeBellis, McDonnell Douglas-East, P.0. Box 516, St. Louis, M0 63166

119. H. W. Deckman, Advanced Energy Systems Laboratory, Government Research Laboratories, Exxon Research and Engineering Co., P. . B. Box 8, Linden, NJ 07036

120. D. A. Dingee, Manager, Fusion Programs, Battelle-Northwest, Battelle Blvd., P.0. Box 399 , Richland, WA 99352

121. H. Urefcer, Gruup CTR-1, Los Mlamos Scientifir. Laboratory, P.0. Box 1663, Los ATamos, NM 87545

122. R. A. Ellis, Plasma Physics Laboratory, Princeton University, P. 0 . Box 451 , Princeton, NJ 08540

123. W. R. Ellis, Jr., Office of Fusion Energy (ETM), Mail Stop G-234, Department of Energy, Washington, DC 20545

124. H. H. Fleischmann, Department of Applied Physics, Clark Hall, Cornell University, Ithaca, NY 14853

125. H. K. For'sen, Exxon Nuclear Co., 777 106th Ave. NE, Bellevue, WA 98009

126. J. Foster, TRW Defense and Space Systems, I Space Park, Bldg. R-1, Redondo Beach, CA 90278

127. T. K. Fowler, Lawrence Livermọre Laboratory, P.0. Box 808, Livermore, CA 94550 
128. M. Fujiwara, Institute of Plasma Physics, Nagoya University, Nagoya 464, Japan

129. H. P. Furth, Plasma Physics Laboratory, Princeton University, P.0. Box 451 , Princeton, NJ 08540

130. Fusion Power Program, Argonne National Laboratory, 9700 S. Cass Ave., Argonne, IL 60439

131. M. B. Gottlieb, Plasma Physics Laboratory, Princeton University, P.0. Box 451, Princeton, NJ 08540

132. R. W. Gould, California Institute of Technology, Mail Stop 116-81, Pasadena, CA 91109

133. H. Grad, New York University, Courant Institute of Mathematical Sciences, Magneto-Fluid Dynamics Division, 251 Mercer St., New York, NY 10012

134. A. Grant, TRW Defense and Space Systems, I Space Park, B1dg. R-1, Redondo Beach, CA 90278

135. G. E. Guest, General Atomic Co., P.0. Box 81608, San Diego, CA 92138

136. E. G. Harris, Department of Physics, University of Tennessee, Knoxville, TN 37916

137. I. G. Hedrick, Mail Stop C-0405, Grumman Aerospace Corp., Bethpage. NY 11714

138. G. K. Hess, Ir., Office of Cusiun Eneryy (ElM), Mail Stop G-234, Department of Energy, Washington, DC 20545

139. R. L. Hickok, Rensselaer Polytechnic Institute, ESE Department, Troy, NY 12181

140. A. G. Hill, Massachusetts Institute of Technology, Cambridge, MA 02739

141. R. L. Hirsch, Exxon Corp., Science and Technology Department, 1251 Avenue of the Americas, New York, NY 10020

142. J. Hosea, Plasma Physics Laboratory, Princeton University, P.0. Box 451, Princeton, NJ 08540

143. 5. Hulllphrles, Laboratory of Plasma Physics, 308 Upson Hall, Cornell University, Ithaca, NY 1405J

144. R. Huse, Chairman, EPRI Fusion Prograin Cuntiltee, Hublic Service and Gas Co.., BO Park Plare, Newark, NJ 07101

145. H. Ikeqami. Institute of Plasma Physics, Nayoya Univerśity, Nagoya 464, Japan

146. H. R. Jory, Varian Associates, 611 Hansen Way, Palo Alto, CA 94303

147. A. Kadish, Office of Fusion Energy (ETM), Mail Stop G-234, Department of Energy, Washington, DC 20545

148. J. Killeen, Lawrence Livermore Laboratory, P.0. Box 808, Livermore, CA 94550

149. E. E. Kintner, Director, Office of Fusion Energy (ETM), Mail Stop G-234, Department. of Energy, Washington, DC 20545

150. N. A. Kral1, Science Applications, Inc., 1200 Prospect St., Box 2351, La Jolla, CA 92037

151. E. Lee, Controlled Thermonuclear Research Division, Lawrence Livermore Laboratory, P.0. Box 808, Livermore, CA 94550

152. Librarian, Institut für Plasmaphysik, 8046 Garching bei Munchen, Federal Republic of Germany

153. Librarian, Culham Laboratory, UK Atomic Energy Authority, Abingdon, Oxfordshire, England

154. Library, Centre de Recherches en Physique des Plasmas, 21 Avenue des Bains, 1007 Lausanne, Switzerland

155. D. G. McAlees, Exxon Nuclear Co., Inc., Research \& Technology Center, Laser Enrichment Department, 2955 George Washington Way, Richland, WA 99352

156. T. Mene, McDonnel1 Douglas-East, P.0. Box 516. St. I n!is, M0 63166

157. R. Moir, Lawiellie Livermore Laboratory, P.0. Box 808, Livermore, CA 91550

158. K. G. Moses, TRW Defense and Space Systems, 1 Space Park, Bldg. R-1, Redondo Beach, CA 90278

159. J. H. Mullen, McDonnell Douglas-East, P.0. Box 516, St. Louis, M0 6.316

160. S. Naymark, Nuclear Scrvices Cürj., 1700 Dell Ave., Campbell, CA 95008

161. T. Ohkawa, Generdl Atomic lin., P.0. Box 81608, San Dieyo, C.A 92138

162. 1. U. Pearlstcin, Lawrence Livermore Laboratenry, P.0. Box 80R, l ivermure, CA 94j'sU

163. Plisma Pliybici Library, Pldsild Physics Laboratory, Princeton University, P.0. Box 451, Princeton, NJ 08540

164. R. E. Price, Chief, Fusion Plasma Theory Branch, Office of Fusion Energy (ETM), Mail Stop G-234, Department of Energy, Washington, DC 20545

165. D. J. Rose, Department of Nurlpar Enginccring, Massacliusetts In̈stıtute of Technology, Cambridge, MA UंI'BY

166. F. R. Scott, Electrir. Pnwer Rescarch Institule, 3112 Hillview Avè., P.0. Box 10412, Pa70 Alto, CA '94304

167. Z. M. Shapiro, Westinghouse Electric Co., P.0. Box 355, Pittsburgh, PA 15230

168. W. M. Stacey, Jr., School of Nuclear Engineering, Georgia Institute of Tcchnology, Allanta, GA 3.3 .32

169. A. Staprans, Varian Associates, 611 Hanson Way, Palu Altu, CA 94303

170. H. S. Státen, Office of Fusion Energy (ETM), Mail Stop G-2.34, Department of Inergy, Washlngron, DC. 20.545

1\%. P. Staudhammer, TRW Defense and Space Systells, I space Park, B1dg. R-1, Redondo Beach, CA 90278

172. C. M. Stickley, Laser Fusion, Department of Energy, Washington, DC 20545

173. R. S. Symons, Varian Associates, 611 Hansen Way, Palo Alto, CA 94303

174. F. Thomas, Grumman Aerospace Corp., Department 930, PLT 5, Bethpage, NY 11714

175. A. W. Trivelpiece, Exxon Nuclear Cn., Inc., Research \& Technulogy Center, 2955 George Washington Way, Richland, WA 99352

176. W. C. Turner, Lawrence Livermore Laboratory, P.0. Box 808, Livermore, CA 94550

177. C. von Keszycki, Grumman Aerospace Corp., Department 930, PLT 5, Bethpage, NY 11714 
178. Director, Research and Technical Support Division, Department of Energy, Oak Ridge Operations, P.O. Box E, Oak Ridge, TN 37830

179-433. Given distribution as shown in TID-4500, Magnetic Fusion Energy (Distribution Category UC-20 f, g: Experimental Plasma Physics and Theoretical Plasma Physics) 